\title{
Azidation of $\beta$-Keto Esters and Silyl Enol Ethers with a Benziodoxole Reagent
}

\author{
Maria Victoria Vita and Jérôme Waser*
}

Laboratory of Catalysis and Organic Synthesis, Institute of Chemical Sciences and

Engineering, Ecole Polytechnique Fédérale de Lausanne, EPFL SB ISIC LCSO, BCH

4306, 1015 Lausanne, Switzerland.

jerome.waser@epfl.ch

Received Date (will be automatically inserted after manuscript is accepted)

ABSTRACT

The efficient azidation of $\beta$-keto esters and silyl enol ethers using a benziodoxole-derived azide transfer reagent is reported. The azidation of cyclic $\beta$-keto esters could be achieved in up to quantitative yields in the absence of any catalyst. In the case of less reactive linear $\beta$-keto esters and silyl enol ethers, complete conversion and good yields could be obtained by using a zinc catalyst.

Azides are extremely useful functional groups in synthetic chemistry. ${ }^{1}$ They are easily reduced to amines, but are themselves non-basic. They consequently constitute ideal amine precursors in multi-step synthesis. Furthermore, cycloaddition of azides with alkynes, especially catalyzed by copper, has become the most impressive example of "Click" chemistry, as it is completely orthogonal to other functionalities and can run under mild conditions without the need to exclude oxygen or moisture. This reaction is now one of the most often used functionalization methods for biomolecules and

(1) (a) Bräse, S.; Gil, C.; Knepper, K.; Zimmermann, V Angew. Chem., Int. Ed. 2005, 44, 5188. (b) Organic Azides: Syntheses and Applications. Bräse, S.; Banert, K. ed.; Wiley: 2009. polymers. ${ }^{2}$ In this context, new methods to introduce azides, especially in complex molecules, are urgently needed.

Most approaches to access azides are based on the use of nucleophilic sodium azide. In contrast, the introduction of azides onto nucleophiles is much less developed. Such reactions would be especially useful to access $\alpha$-azido carbonyl compounds, which are important building

(2) (a) Rostovtsev, V. V.; Green, L. G.; Fokin, V. V.; Sharpless, K. B. Angew. Chem., Int. Ed. 2002, 41, 2596. (b) Tornoe, C. W.; Christensen, C.; Meldal, M. J. Org. Chem. 2002, 67, 3057. Reviews: (c) Kolb, H. C.; Sharpless, K. B. Drug Discovery Today 2003, 8, 1128. (d) Lutz, J.-F. Angew. Chem., Int. Ed. 2007, 46, 1018. (e) Moses, J. E.; Moorhouse, A. D. Chem. Soc. Rev. 2007, 36, 1249. (f) Meldal, M.; Tornoe, C. W. Chem. Rev. 2008, 108, 2952. (g) Amblard, F.; Cho, J. H.; Schinazi, R. F. Chem. Rev. 2009, 109, 4207. (h) Iha, R. K.; Wooley, K. L.; Nystrom, A. M.; Burke, D. J.; Kade, M. J.; Hawker, C. J. Chem. Rev. 2009, 109, 5620 . 
blocks in synthetic chemistry as precursors of amino acid derivatives. ${ }^{3}$ Sulfonyl azides have been most often used as electrophilic azide sources, but they work best for strongly nucleophilic lithium enolates. ${ }^{4}$ For the stabilized enolates derived from dicarbonyl compounds, a mixture of products resulting from competing diazo transfer and subsequent rearrangements has been observed. ${ }^{5}$ Another successful approach is based on the in situ Umpolung of either the azide or the enolate using a strong oxidant. These reactions often involve the formation of iodoazide or hypervalent iodine reagents, and have been usually proposed to proceed via a radical pathway. ${ }^{6}$ In the case of the azidation of dicarbonyl compounds, the most general protocol has been reported in 2012 by Kirsch and coworkers based on the combination of sodium azide and IBX. $^{6 n}$ In most oxidative methods, the actual azidation reagent is either unknown, or so unstable that it cannot be isolated. This makes the development of catalytic or asymmetric methods very difficult. In fact, until 2013, no catalytic asymmetric $\alpha$-azidation of carbonyl compounds had been reported, and the only method to access enantiopure products was based on a chiral auxiliary approach. ${ }^{4}$

To solve this challenge, we decided to make use of the exceptional stability of benziodoxol(on)e-based hypervalent iodine reagents. ${ }^{7}$ Our group has recently demonstrated the exceptional properties of this class of reagents for acetylene transfer onto dicarbonyl compounds, ${ }^{8}$ and we wondered if similar azido reagents would be equally successful. In fact, Zhdankin and coworkers have described the synthesis of azido benziodoxol(on)e compounds, which were surprisingly stable. ${ }^{9}$ The reactivity of these reagents was studied for

(3) Patonay, T.; Konya, K.; Juhasz-Toth, E. Chem. Soc. Rev. 2011, 40, 2797.

(4) Evans, D. A.; Britton, T. C. J. Am. Chem. Soc. 1987, 109, 6881.

(5) (a) Benati, L.; Calestani, G.; Nanni, D.; Spagnolo, P. J. Org. Chem. 1998, 63, 4679. (b) Benati, L.; Nanni, D.; Spagnolo, P. J. Org. Chem. 1999, 64, 5132.

(6) (a) Fowler, F. W.; Hassner, A.; Levy, L. A. J. Am. Chem. Soc. 1967, 89, 2077. (b) Moriarty, R. M.; Vaid, R. K.; Ravikumar, V. T.; Vaid, B. K.; Hopkins, T. E. Tetrahedron 1988, 44, 1603. (c) Magnus, P.; Barth, L. Tetrahedron Lett. 1992, 33, 2777. (d) Magnus, P.; Lacour, J J. Am. Chem. Soc. 1992, 114, 767. (e) Magnus, P.; Hulme, C.; Weber, W. J. Am. Chem. Soc. 1994, 116, 4501. (f) Magnus, P.; Barth, L. Tetrahedron 1995, 51, 11075. (g) Magnus, P.; Lacour, J.; Evans, P. A.; Roe, M. B.; Hulme, C. J. Am. Chem. Soc. 1996, 118, 3406. (h) Magnus, P.; Lacour, J.; Evans, P. A.; Rigollier, P.; Tobler, H. J. Am. Chem. Soc. 1998, 120, 12486. (i) Tohma, H.; Egi, M.; Ohtsubo, M.; Watanabe, H.; Takizawa, S.; Kita, Y. Chem. Commun. 1998, 173. (j) Lee, J. C.; Kim, S.; Shin, W. C. Synth. Commun. 2000, 30, 4271. (k) Pedersen, C. M.; Marinescu, L. G.; Bols, M. Org. Biomol. Chem. 2005, 3, 816. (1) Kumar, D.; Sundaree, S.; Rao, V. S. Synth. Commun. 2006, 36, 1893. (m) Telvekar, V. N.; Patile, H. V. Synth. Commun. 2011, 41, 131. (n) Harschneck, T.; Hummel, S.; Kirsch, S. F.; Klahn, P. Chem. Eur. J. 2012, 18, 1187.

(7) (a) Zhdankin, V. V. Curr. Org. Synth. 2005, 2, 121. (b) Brand, J. P.; Fernandez Gonzalez, D.; Nicolai, S.; Waser, J. Chem. Commun. 2011, 47, 102.

(8) Fernandez Gonzalez, D.; Brand, J. P.; Waser, J. Chem. Eur. J. $2010,16,9457$.

(9) (a) Zhdankin, V. V.; Kuehl, C. J.; Krasutsky, A. P.; Formaneck, M. S.; Bolz, J. T. Tetrahedron Lett. 1994, 35, 9677. (b) Krasutsky, A. P.; Kuehl, C. J.; Zhdankin, V. V. Synlett 1995, 1081. (c) Zhdankin, V. V.; Krasutsky, A. P.; Kuehl, C. J.; Simonsen, A. J.; Woodward, J. K.; Mismash, B.; Bolz, J. T. J. Am. Chem. Soc. 1996, 118, 5192. the radical-based azidation of aliphatic $\mathrm{C}-\mathrm{H}$ bonds. ${ }^{9}{ }^{910}$ During completion of our work, Gade and co-workers reported the first catalytic asymmetric method for the azidation of $\beta$-keto esters and oxindoles using a chiral iron catalyst together with an azidobenziodoxole reagent. ${ }^{11}$ This major breakthrough motivate us to report our own preliminary results in the area, including the very efficient azidation of cyclic $\beta$-keto esters with azidobenziodoxole $\mathbf{2}$ in the absence of any catalyst and the zinc-catalyzed azidation of less reactive linear keto esters and silyl enol ethers using the same reagent (Scheme 1). Our results demonstrated that the potential of azidobenziodoxole reagents as electrophilic azidation reagents is not limited to cyclic $\beta$-keto esters.

Scheme 1. Azidation of Keto Esters and Silyl Enol Ethers

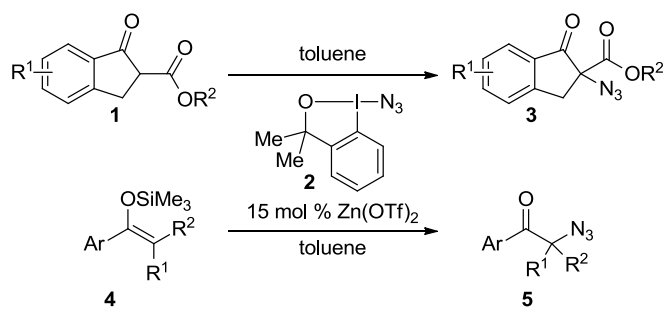

We started our studies with the investigation of the azidation of keto ester 1a with benzidoxol(on)e reagents $\mathbf{2}$ and 6. Whereas no reaction was observed with benziodoxolone $\mathbf{6}$, a complete conversion to azide $\mathbf{3 a}$ was observed with benziodoxole 2 in different solvents, with the best yield ( $82 \%$ by ${ }^{1} \mathrm{H}$ NMR) obtained in toluene. ${ }^{12}$ The better result obtained with benziodoxole $\mathbf{2}$ is probably due to the higher basicity of the alcoholate generated after azide transfer.

Scheme 2. Azidation of Keto Ester 1a.

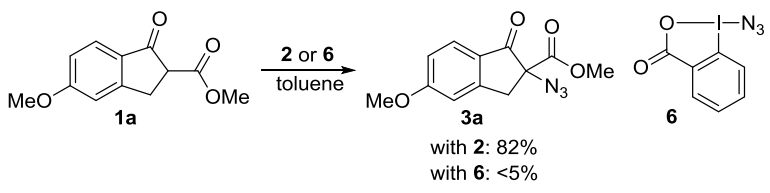

With this extremely simple protocol for the azidation of keto esters in hand, we shortly investigated the scope of the reaction (Table 1). On a $0.40 \mathrm{mmol}$ scale, azide 3a could be isolated in $94 \%$ yield (entry 1 ). The azidation

(10) In their first communication, ${ }^{9 \mathrm{a}}$ Zhdankin and co-workers indicated that these reagents may also react with nucleophiles derived from carbonyls, but the result of such reactions was never reported.

(11) Deng, Q. H.; Bleith, T.; Wadepohl, H.; Gade, L. H. J. Am. Chem. Soc. 2013, 135, 5356.

(12) Yield obtained in other solvents: $\mathrm{CH}_{2} \mathrm{Cl}_{2}: 79 \%, \mathrm{CH}_{3} \mathrm{CN}: 75 \%$, $\mathrm{Et}_{2} \mathrm{O}$ : $69 \%$, THF: $63 \%,{ }^{i} \mathrm{PrOH}: 49 \%$. See Supporting Information for further detail. 
proceeded in good yields independent of the electronic properties of the substituent on the benzene ring of indanone (entries 2-4). More sterically hindered esters gave quantitative yields of azide (entries 5 and 6). Azidation of keto amide $1 \mathrm{~g}$ was also possible in $67 \%$ yield (entry 7). ${ }^{13}$ Nevertheless, the reaction did not work for less reactive substrates, and no conversion was observed for both acyclic keto ester $\mathbf{1 h}$ (entry 8 ) or silyl enol ether $\mathbf{4 a}$ (entry 9).

Table 1. Azidation of cyclic $\beta$-keto esters with benziodoxole 2 .

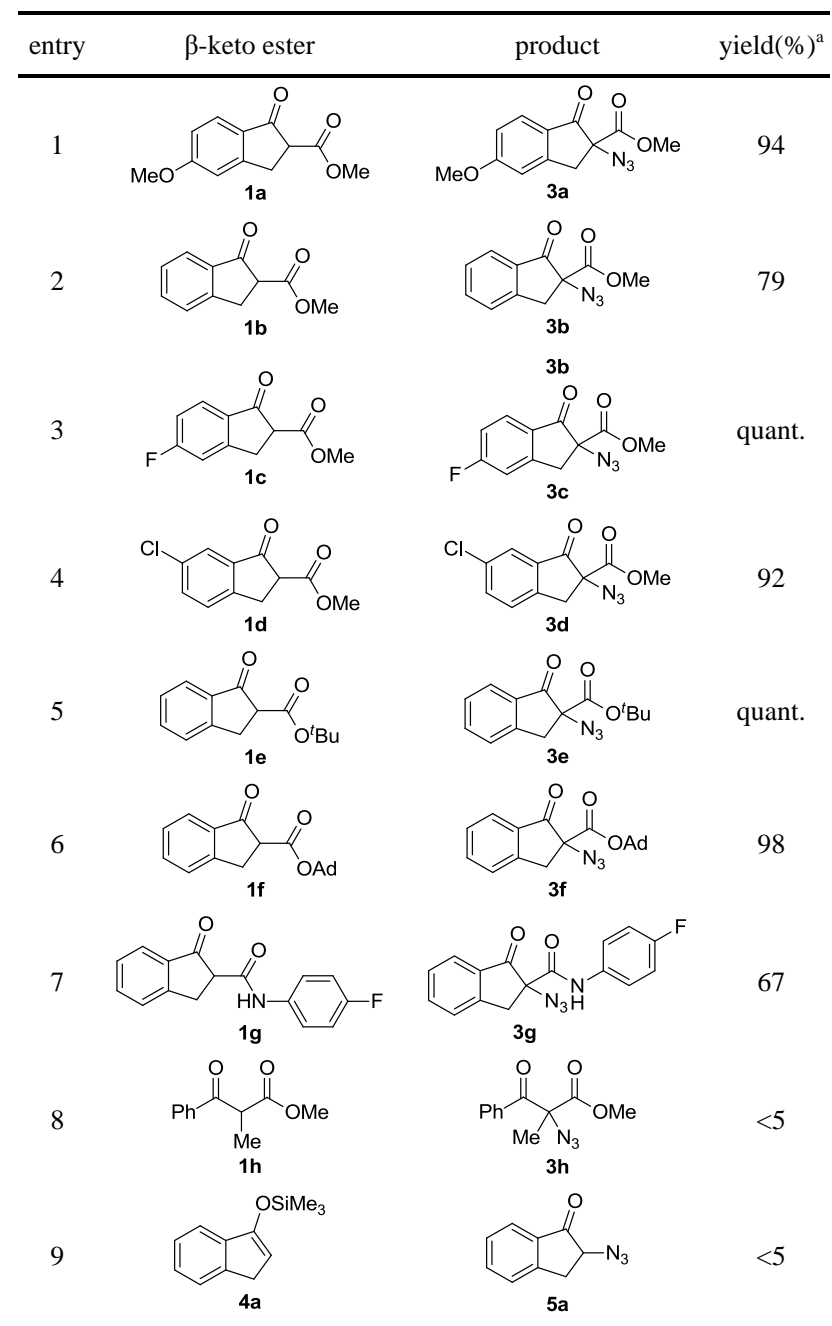

${ }^{\mathrm{a}}$ Isolated yields with keto ester $\mathbf{1}$ or enol ether $\mathbf{4 a}(0.40 \mathrm{mmol})$, benziodoxole $2(0.52 \mathrm{mmol})$, toluene $(0.4 \mathrm{~mL}), 1-3 \mathrm{~h}$ at rt.

The low reactivity of $\mathbf{2}$ with silyl enol ethers is contrasting with the fast reaction reported for in situ generated non cyclic reagents. ${ }^{6-h}$ We wondered if the reactivity of the reagent could be further enhanced in the presence of a Lewis acid. In fact, several Lewis acids were able to catalyze the reaction of silyl enol ether $\mathbf{4 a}$

(13) In the case of a tetralone-derived keto-ester, only a low yield $(10 \%)$ of azidation was obtained, due to the formation of an aromatic product. A simple cyclopentanone could also be azidated in moderate yield (54\%). See Supporting Information for further details. with benziodoxole 2 in toluene (Table 2). Moderate yields were obtained with $\mathrm{Sc}(\mathrm{OTf})_{3}, \mathrm{Cu}(\mathrm{OTf})_{2}$ and $\mathrm{In}(\mathrm{OTf})_{3}$ as catalysts (entries 1-3). The best results were obtained when using $\mathrm{Sn}(\mathrm{OTf})_{2}, \mathrm{Hf}(\mathrm{OTf})_{4} \bullet 4 \mathrm{H}_{2} \mathrm{O}$ or $\mathrm{Zn}(\mathrm{OTf})_{2}$, which gave nearly identical yields $(67-71 \%$, entries 4 $6)$.When considering that zinc is cheap and non-toxic, we decided to continue with this metal as catalyst. Other zinc salts gave inferior results (entries 7-9). Triflic acid was also able to catalyze the reaction, but in only $28 \%$ yield (entry 10). Lower yields were also obtained in solvent different from toluene (entries 11-15). Finally, more sterically hindered silyl enol ethers $\mathbf{4 b}$ and $\mathbf{4 c}$ led to inferior results when compared to trimethylsilyl enol ether 4a (entries 16 and 17). We consequently decided to examine the scope of the reaction with trimethysilyl enol ethers and zinc triflate as catalyst (Table 3, entries 1-9).

Table 2. Optimization of the azidation of silyl enol ethers.

\begin{tabular}{|c|c|c|c|c|}
\hline entry & $\mathrm{R}$ & catalyst & solvent & $\operatorname{yield}(\%)^{\mathrm{a}}$ \\
\hline 1 & $\operatorname{Me}(\mathbf{4 a})$ & $\mathrm{Sc}(\mathrm{OTf})_{3}$ & toluene & 23 \\
\hline 2 & $\operatorname{Me}(\mathbf{4 a})$ & $\mathrm{Cu}(\mathrm{OTf})_{2}$ & toluene & 36 \\
\hline 3 & $\operatorname{Me}(\mathbf{4 a})$ & $\operatorname{In}(\mathrm{OTf})_{3}$ & toluene & 58 \\
\hline 4 & $\operatorname{Me}(4 a)$ & $\mathrm{Sn}(\mathrm{OTf})_{2}$ & toluene & 68 \\
\hline 5 & $\operatorname{Me}(\mathbf{4 a}))$ & $\mathrm{Hf}(\mathrm{OTf})_{4} \bullet 4 \mathrm{H}_{2} \mathrm{O}$ & toluene & 71 \\
\hline 6 & $\operatorname{Me}(\mathbf{4 a})$ & $\mathrm{Zn}(\mathrm{OTf})_{2}$ & toluene & 67 \\
\hline 7 & $\operatorname{Me}(\mathbf{4 a})$ & $\mathrm{Zn}\left(\mathrm{NTf}_{2}\right)_{2}$ & toluene & 41 \\
\hline 8 & $\operatorname{Me}(\mathbf{4 a})$ & $\mathrm{Zn}(\mathrm{OAc})_{2}$ & toluene & 22 \\
\hline 9 & $\operatorname{Me}(4 a)$ & $\mathrm{Zn}\left(\mathrm{BF}_{4}\right)_{2}$ & toluene & 47 \\
\hline 10 & $\operatorname{Me}(\mathbf{4 a})$ & HOTf & toluene & 28 \\
\hline 11 & $\operatorname{Me}(\mathbf{4 a}))$ & $\mathrm{Zn}(\mathrm{OTf})_{2}$ & $\mathrm{CH}_{3} \mathrm{CN}$ & 53 \\
\hline 12 & $\operatorname{Me}(\mathbf{4 a})$ & $\mathrm{Zn}(\mathrm{OTf})_{2}$ & $\mathrm{Et}_{2} \mathrm{O}$ & 52 \\
\hline 13 & $\operatorname{Me}(\mathbf{4 a})$ & $\mathrm{Zn}(\mathrm{OTf})_{2}$ & $\mathrm{CH}_{2} \mathrm{Cl}_{2}$ & 30 \\
\hline 14 & $\operatorname{Me}(\mathbf{4 a}))$ & $\mathrm{Zn}(\mathrm{OTf})_{2}$ & THF & 26 \\
\hline 15 & $\operatorname{Me}(\mathbf{4 a}))$ & $\mathrm{Zn}(\mathrm{OTf})_{2}$ & ${ }^{i} \mathrm{PrOH}$ & 0 \\
\hline 16 & Et $(\mathbf{4 b})$ & $\mathrm{Zn}(\mathrm{OTf})_{2}$ & toluene & 44 \\
\hline 17 & $\left.{ }^{i} \operatorname{Pr}(4 c)\right)$ & $\mathrm{Zn}(\mathrm{OTf})_{2}$ & toluene & 23 \\
\hline
\end{tabular}

${ }^{\text {a }} 4$ (0.1 mmol), 2 (0.13 mmol), catalyst (15 mol \%), solvent (2 mL); Yield obtained by ${ }^{1} \mathrm{H}$ NMR using 1,3,5-trimethoxybenzene as standard.

On a $0.40 \mathrm{mmol}$ scale, the azidation worked for silyl enol ethers derived from indanone and tetralone (Table 3, entries 1 and 2). Complete conversion was achieved in a few hours. The reaction could also be applied to the synthesis of tertiary azides (entries 3 and 4). The silyl enol ethers of acetophenone derivatives also worked in the reaction (entries 5-8), giving access to both primary and secondary azides. The azidation also proceeded well in presence of a thiophene heterocycle (entry 9).

At this point, we wondered if the zinc-catalyzed conditions could also be used to extend the scope of azidation for keto ester substrates. Indeed, acyclic keto ester 1h, which did not react in the presence of azidobenziodoxole 2 only, could be now azidated in 73\% yield (Table 3, entry 10). The reaction was also successful for keto esters bearing a more functionalized benzene ring (entries 11 and 12) or a thiophene heterocycle (entry 13). 
Cyclic keto esters are a privileged class of substrates for the development of asymmetric reactions, as twopoints binding of a chiral catalyst can lead to good enantioselectivity. However, the high background rate observed with reagent 2 for cyclic keto esters constituted an important challenge for asymmetric induction. Nevertheless, an interesting preliminary result was obtained using a copper catalyst and bisoxazoline 7 as chiral ligand for the azidation of keto ester 1e with $49 \%$ ee (Scheme 3). The results of Gade and co-workers obtained simultaneously to our own investigations demonstrated the higher efficiency of a tridentate bisoxazoline ligand with iron as catalyst. ${ }^{11}$

Table 3. Scope of the zinc-catalyzed azidation.

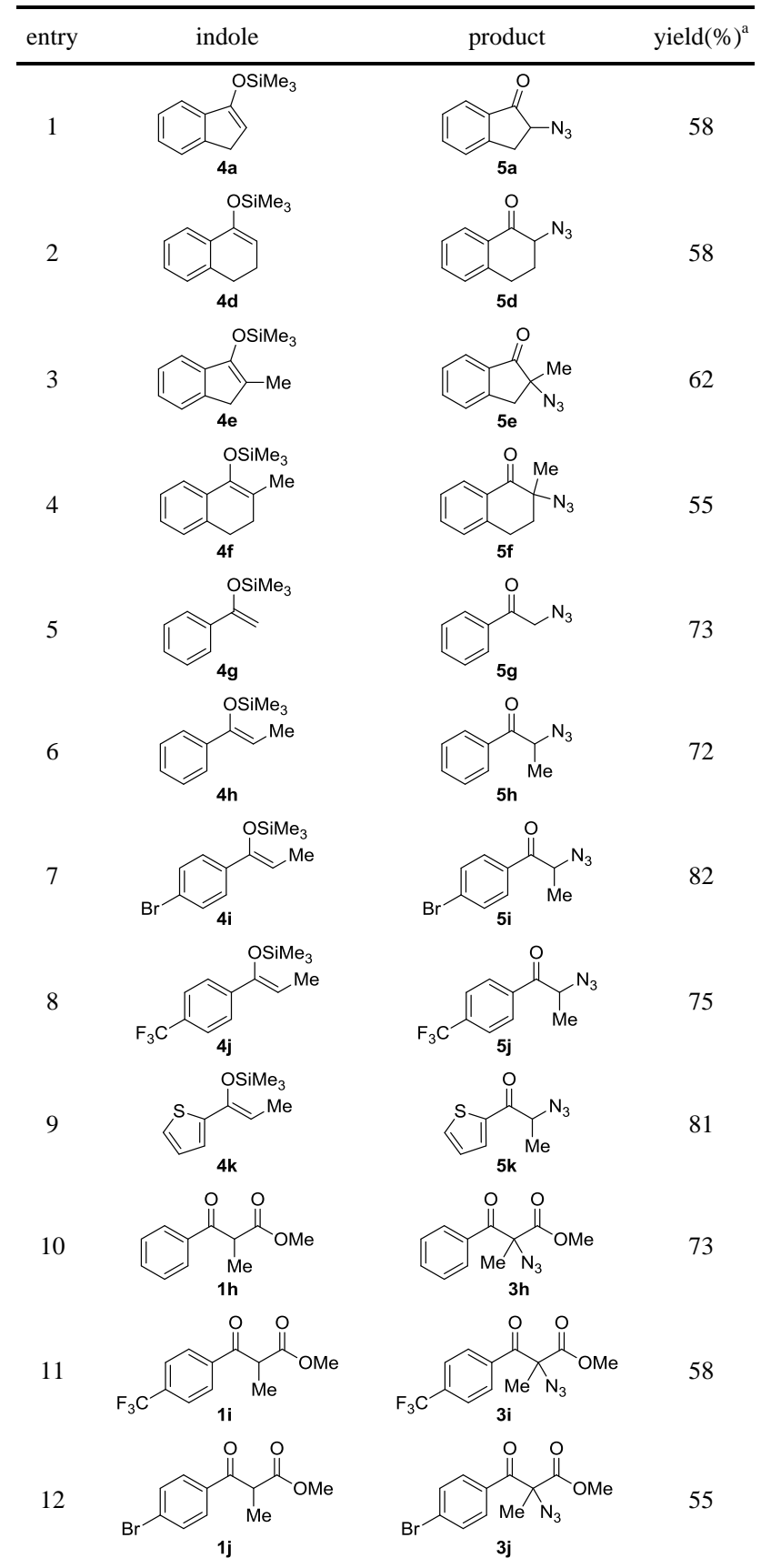

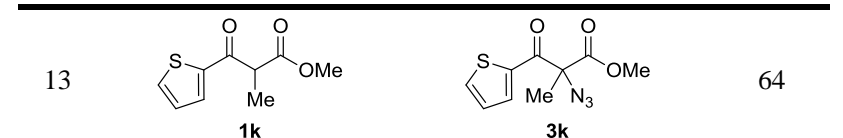

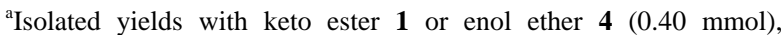
benziodoxole $2(0.52 \mathrm{mmol}), \mathrm{Zn}(\mathrm{OTf})_{2}(0.060-0.12 \mathrm{mmol})$, toluene $(0.8$ $\mathrm{mL}), 12 \mathrm{~h}$ at rt.

Scheme 3. Enantioselective Azidation of Keto-Ester 1e.

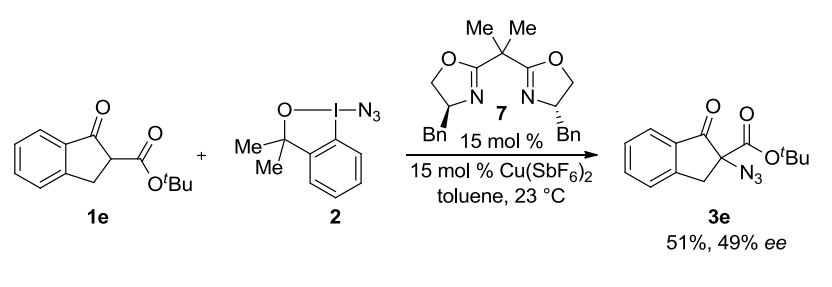

Several mechanisms can be envisaged for the azidation reaction. A first possibility is nucleophilic attack of either the oxygen or the carbon atom of the enolate on the iodine atom of benziodoxole 2 , followed by $\mathrm{C}-\mathrm{N}$ bond formation. A second alternative would involve a radical chain reaction, as has been proposed by Magnus and coworkers. ${ }^{6 c-h}$ However, several observations make the latter less probable: in contrast to what was reported by Magnus and co-workers, no reaction was observed in the absence of catalyst. Furthermore, diazidation products resulting from radical pathways were not obtained, and the reaction did not require the presence of a bulky triisopropylsilyl group on the oxygen. Finally, the addition of TEMPO to the reaction mixture had no influence on the reaction outcome, neither with keto esters nor with silyl enol ethers. These results are consequently more in accordance with an electrophilic azidation, as has been proposed recently by Muniz and co-workers for the transfer of NTs group using an hypervalent iodine reagent. ${ }^{14}$ The role of the zinc catalyst is also intriguing, as it may act either through nucleophilic activation by formation of a zinc enolate, or by electrophilic activation of benziodoxole 2. Further investigations will be required to gain a better understanding of the reaction mechanism.

In conclusion, we have described that the azidation of cyclic keto esters with benziodoxole reagent 2 proceeded in excellent yield in the absence of any catalyst. For less reactive acyclic keto esters and silyl enol ethers, an efficient zinc-catalyzed method could be developed, which gave access to primary, secondary and tertiary azides. Further extension of the scope of the reaction and development of asymmetric protocols not limited to

(14) Souto, J. A.; Martinez, C.; Velilla, I.; Muniz, K. Angew. Chem., Int. Ed. 2013, 52, 1324 
cyclic keto esters are currently under investigation and the results will be reported in due course.

Supporting Information. Experimental procedures and analytical data for all new compounds. This material is available free of charge via the Internet at http://pubs.acs.org.

Acknowlegment. We thank the EPFL and SNF (grant 200020_134550) for funding and F. Hoffmann-La Roche Ltd for an unrestricted research grant. 


\title{
Supporting Information
}

\author{
111 pages
}

\section{Azidation of $\beta$-Keto Esters and Silyl Enol Ethers with a Benziodoxole Reagent.}

\author{
Maria Victoria Vita and Jérôme Waser
}

Laboratory of Catalysis and Organic Synthesis, Institute of Chemical Sciences and Engineering, Ecole Polytechnique Fédérale de Lausanne, EPFL SB ISIC LCSO, BCH 4306, 1015 Lausanne, Switzerland.

jerome.waser@epfl.ch 


\section{Table of contents}

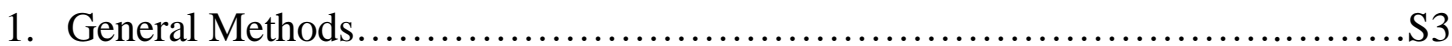

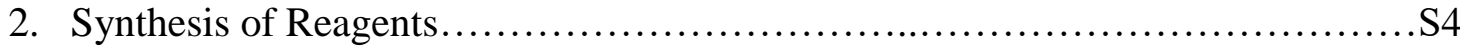

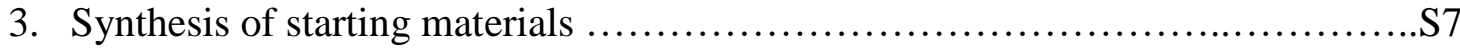

4. Azidation of $\beta$-keto esters.............................................. 15

5. Zinc-catalyzed azidation of linear $\beta$-keto esters.......................... 17

6. Optimization of the azidation of silyl enol ethers............................ 19

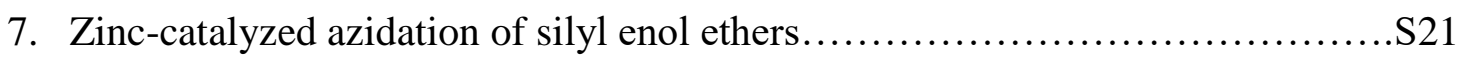

8. Enantioselective azidation of $\beta$-keto esters.............................. 26

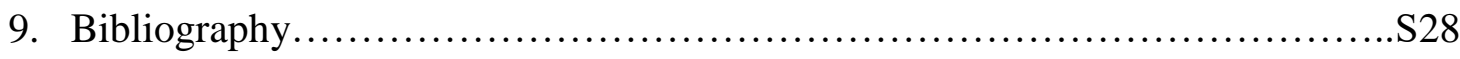

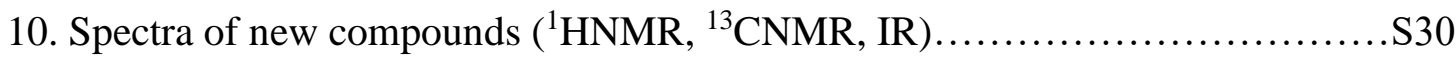




\section{General Methods}

All reactions were carried out in oven dried glassware under an atmosphere of nitrogen, unless stated otherwise. For quantitative flash chromatography technical grade solvents were used. For flash chromatography for analysis, HPLC grade solvents from Sigma-Aldrich were used. THF, $\mathrm{Et}_{2} \mathrm{O}, \mathrm{CH}_{3} \mathrm{CN}$, toluene, hexane and $\mathrm{CH}_{2} \mathrm{Cl}_{2}$ were dried by passage over activated alumina under nitrogen atmosphere $\left(\mathrm{H}_{2} \mathrm{O}\right.$ content $<10 \mathrm{ppm}$, Karl-Fischer titration). All chemicals were purchased from Acros, Aldrich, Fluka, VWR, Fluorochem, Aplichem or Merck and used without further purification, unless stated otherwise. Chromatographic purification was performed as flash chromatography using Macherey-Nagel silica 40-63, $60 \AA$, using the solvents indicated as eluent with 0.1-0.5 bar pressure. TLC was performed on Merck silica gel $60 \mathrm{~F}_{254}$ TLC aluminium plates and visualized with UV light and anisaldehyde stain. Melting points were measured on a calibrated Büchi B-540 melting point apparatus using open glass capillaries. ${ }^{1} \mathrm{H}-\mathrm{NMR}$ spectra were recorded on a Bruker DPX-400 $400 \mathrm{MHz}$ spectrometer in chloroform-d and/or DMSO- $\mathrm{d}_{6}$. All signals are reported in ppm with the internal chloroform signal at $7.26 \mathrm{ppm}$ or the internal DMSO signal at $2.50 \mathrm{ppm}$ as standard. The data is being reported as $(\mathrm{s}=$ singlet, $\mathrm{d}=$ doublet, $\mathrm{t}=$ triplet, $\mathrm{q}=$ quadruplet, $\mathrm{qi}=$ quintet, $\mathrm{m}=$ multiplet or unresolved, $\mathrm{br}=$ broad signal, app $=$ apparent, coupling constant(s) in $\mathrm{Hz}$, integration, interpretation) ${ }^{13} \mathrm{C}-\mathrm{NMR}$ spectra were recorded with ${ }^{1} \mathrm{H}$-decoupling on a Bruker DPX-400 $100 \mathrm{MHz}$ spectrometer in chloroform-d and/or DMSO- $\mathrm{d}_{6}$. All signals are reported in ppm with the internal chloroform signal at $77.0 \mathrm{ppm}$ or the internal DMSO signal at $39.5 \mathrm{ppm}$ as standard. Infrared spectra were recorded on a JASCO FTIR B4100 spectrophotometer with an ATR PRO410-S and a ZnSe prisma and are reported as $\mathrm{cm}^{-}$

${ }^{1}$ ( $\mathrm{w}=$ weak, $\mathrm{m}=$ medium, $\mathrm{s}=$ strong, $\mathrm{br}=$ broad). Gas chromatographic and low resolution mass spectrometric measurements were performed on a Perkin-Elmer Clarus 600 gas chromatographer and mass spectrometer using a Perkin-Elmer Elite fused silica column (length: $30 \mathrm{~m}$, diameter: $0.32 \mathrm{~mm}$ ) and Helium as carrier gas. High resolution mass spectrometric measurements were performed by the mass spectrometry service of ISIC at the EPFL on a MICROMASS (ESI) Q-TOF Ultima API. Compound 7 was purchase from Aldritch. 


\section{Synthesis of Reagents}

\subsection{Synthesis of 1-azido-3,3-dimethyl-3-(1H)-1,2-benziodoxole (2)}

\section{1-Chloro-1,3-dihydro-3,3-dimethyl-1,2-benziodoxole (9)}
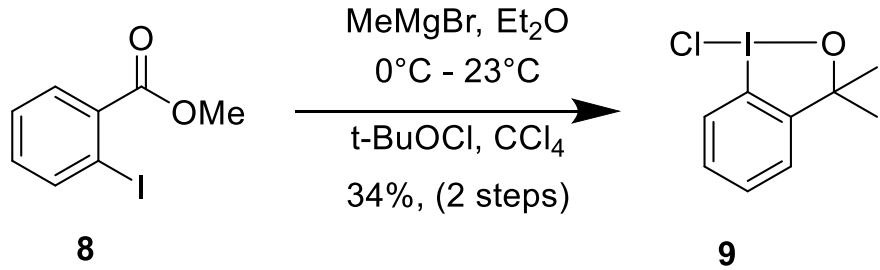

Following a reported procedure ${ }^{1}$ methyl 2-iodobenzoate $(8)(12 \mathrm{~mL}, 76 \mathrm{mmol})$ was dissolved under $\mathrm{N}_{2}$ atmosphere in dry diethyl ether $(400 \mathrm{~mL})$ and then the solution was cooled down at 0 ${ }^{\circ} \mathrm{C}$ with an ice bath. Methylmagnesium bromide $(56.0 \mathrm{~mL}, 168 \mathrm{mmol}, 2.20$ equiv) was added dropwise and the reaction was stirred for $30 \mathrm{~min}$ at $0{ }^{\circ} \mathrm{C}$. The reaction mixture was then allowed to warm to room temperature and it was further stirred for $2 \mathrm{~h}$. The reaction was quenched with $\mathrm{NH}_{4} \mathrm{Cl}$ in an iced bath. The organic layer was separated and extracted with $\mathrm{Et}_{2} \mathrm{O}(3 \mathrm{x} 100 \mathrm{~mL})$, water $(2$ x $200 \mathrm{~mL})$, brine $(1 \times 100 \mathrm{~mL})$ and the combined organic layers were dried over $\mathrm{MgSO}_{4}$. The solvent was removed in vacuum.

With no further purification the crude mixture was dissolved in $\mathrm{CCl}_{4}(7 \mathrm{~mL})$ and tert-butyl hypochlorite $(10 \mathrm{~mL}, 92 \mathrm{mmol}, 1.2$ equiv) and the reaction mixture was stirred at room temperature. After one hour a yellow precipitate was collected by filtration and washed with hexane $(60 \mathrm{~mL})$ to afford compound $9(7.7 \mathrm{~g}, 26 \mathrm{mmol}, 34 \%$ yield $)$ as a yellow solid.

${ }^{1} \mathbf{H}$ NMR (400 MHz, $\left.\mathrm{CDCl}_{3}\right) \delta 8.03(\mathrm{dd}, 1 \mathrm{H}, J=8.1,1.1 \mathrm{~Hz}, \mathrm{CHAr}), 7.55(\mathrm{~m}, 2 \mathrm{H}, \mathrm{CHAr}), 7.17$ (dd, $1 \mathrm{H}, J=7.3,1.7 \mathrm{~Hz}, \mathrm{CHAr}), 1.55\left(\mathrm{~s}, 6 \mathrm{H},\left(\mathrm{CH}_{3}\right)_{2}\right) .{ }^{13} \mathbf{C} \mathbf{N M R}\left(101 \mathrm{MHz}, \mathrm{CDCl}_{3}\right) \delta 149.5$, 131.0, 130.5, 128.4, 126.1, 114.7, 85.2, 29.2. IR $v_{\max } 3729(\mathrm{w}), 3626(\mathrm{w}), 2972(\mathrm{w}), 2924(\mathrm{w})$, $2362(w), 2055(\mathrm{w}), 2018(\mathrm{w}), 1742$ (w), $1564(\mathrm{w}), 1464(\mathrm{w}), 1439(\mathrm{w}), 1379(\mathrm{w}), 1378(\mathrm{w})$, $1366(\mathrm{w}), 1277(\mathrm{w}), 1276(\mathrm{w}), 1256(\mathrm{w}), 1181(\mathrm{w}), 1154(\mathrm{~m}), 1112(\mathrm{w}), 1048(\mathrm{w}), 1003(\mathrm{w}), 982$ (w), $943(\mathrm{~m}), 866(\mathrm{~m}), 808(\mathrm{w}), 790(\mathrm{w}), 789(\mathrm{w}), 762(\mathrm{~s}), 745(\mathrm{w}), 724(\mathrm{w}), 718(\mathrm{w})$. NMR data correspond to the reported values. ${ }^{1}$

\section{1-Acetoxy-1,3-dihydro-3,3-dimethyl-1,2-benziodoxole (10)}

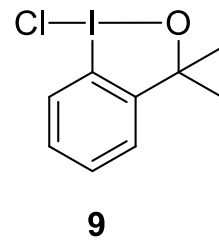

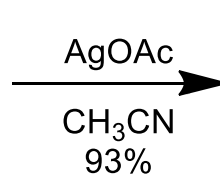

$93 \%$

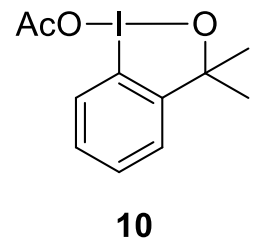

10

Following a reported procedure ${ }^{1}$ 1-Chloro-1,3-dihydro-3,3-dimethyl-1,2-benziodoxole (9) (2.60 $\mathrm{g}, 8.77 \mathrm{mmol}$ ) was dissolved in dry acetonitrile $(25 \mathrm{~mL})$ under $\mathrm{N}_{2}$ atmosphere. The reaction flask was covered with aluminum foils and protected from light. Silver acetate $(1.46 \mathrm{~g}, 8.77 \mathrm{mmol}$, 1.00 equiv) was then added in one portion. The reaction mixture was stirred in the dark at room temperature for $16 \mathrm{~h}$. Filtration over a Celite plug and evaporation of the solvent yielded compound 10 (2.6 g, $8.8 \mathrm{mmol}, 93 \%)$ as a light brownish solid.

${ }^{1} \mathbf{H}$ NMR $\left(400 \mathrm{MHz}, \mathrm{CDCl}_{3}\right) \delta 7.79(\mathrm{dd}, 1 \mathrm{H}, J=8.0,1.3 \mathrm{~Hz}, \mathrm{CHAr}), 7.47$ (m, 2H, CHAr), 7.18 (dd, $1 \mathrm{H}, J=7.2,1.7 \mathrm{~Hz}, \mathrm{CHAr}), 2.11\left(\mathrm{~s}, 3 \mathrm{H}, \mathrm{COCH}_{3}\right), 1.52\left(\mathrm{~s}, 6 \mathrm{H},\left(\mathrm{CH}_{3}\right)_{2}\right) .{ }^{13} \mathrm{C}$ NMR $(101$ 
$\left.\mathrm{MHz}, \mathrm{CDCl}_{3}\right) \delta 177.4,149.4,130.4,130.0,129.9,126.2,115.7,84.6,29.2,21.5 . \mathbf{I R} v_{\max } 3099$ (w), $3057(w), 2975(w), 2930(w), 2930(w), 2865(w), 1740(w), 1640(s), 1588(w), 1566(w)$, 1462 (w), 1438 (m), 1363 (s), 1294 (s), 1259 (m), 1158 (m), 1114 (w), 1047 (w), $1033(\mathrm{w}), 1009$ $(\mathrm{w}), 949(\mathrm{~m}), 926(\mathrm{w}), 866(\mathrm{w}), 761(\mathrm{~s}), 723(\mathrm{w})$. NMR data correspond to the reported values. ${ }^{4}$

\section{1-Azido-3,3-dimethyl-3-(1H)-1,2-benziodoxole (2)}

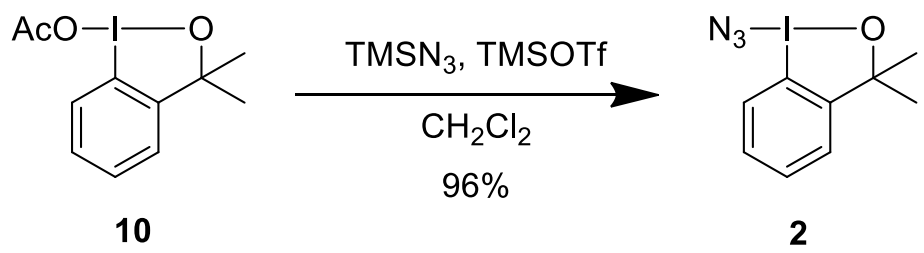

Caution: This reaction should be carried out behind a safety shield! Following a reported procedure $^{2}$ 1-Acetoxy-1,3-dihydro-3,3-dimethyl-1,2-benziodoxole (10) $(2.30 \mathrm{~g}, 7.18 \mathrm{mmol})$ was dissolved in dry $\mathrm{CH}_{2} \mathrm{Cl}_{2}(36 \mathrm{~mL})$ under $\mathrm{N}_{2}$ atmosphere. The reaction was placed in an iced bath and trimethylsilylazide $(0.954 \mathrm{~mL}, 7.18 \mathrm{mmol}, 1.00$ equiv) was added via syringe, followed by TMSOTf ( $0.065 \mathrm{~mL}, 0.36 \mathrm{mmol}, 0.050$ equiv). The reaction was stirred for $15 \mathrm{~min}$ then the ice bath was removed and the stirring was continued for $1 \mathrm{~h}$. The solvent was evaporated and the solid obtained was washed with $n$-hexane $(2 \times 30 \mathrm{~mL}$, HLPC purity) to afford 2 as a yellow crystalline solid ( $2.10 \mathrm{~g}, 7.18 \mathrm{mmol}, 96 \%)$.

${ }^{1} \mathbf{H}$ NMR (400 MHz, $\left.\mathrm{CDCl}_{3}\right) \delta 7.77$ (d, $\left.1 \mathrm{H}, J=8.0 \mathrm{~Hz}, \mathrm{C} \underline{H} \mathrm{Ar}\right), 7.55$ (m, $\left.2 \mathrm{H}, \mathrm{C} \underline{H} \mathrm{Ar}\right), 7.23$ (d, 1 $\mathrm{H}, J=7.2 \mathrm{~Hz}, \mathrm{C} \underline{H} \mathrm{Ar}), 1.53\left(\mathrm{~s}, 7 \mathrm{H},\left(\mathrm{C}_{3}\right)_{2} .{ }^{13} \mathrm{C}\right.$ NMR $\left(101 \mathrm{MHz}, \mathrm{CDCl}_{3}\right) \delta 149.2,130.9,130.4$, 127.8, 126.8, 114.0, 83.2, 29.6. IR $v_{\max } 3254$ (w), 3085 (w), 3051 (w), $2976(\mathrm{w}), 2928$ (w), 2860 (w), $2486(w), 2026$ (s), 1918 (w), 1764 (w), 1697 (w), 1651 (w), 1589 (w), $1562(w), 1462(\mathrm{~m})$, 1428 (m), 1380 (w), 1364 (w), 1312 (w), 1273 (w), 1248 (s), 1182 (w), 1151 (m), $1111(\mathrm{~m}), 1031$ (w), $1004(\mathrm{w}), 943(\mathrm{~m}), 910(\mathrm{~m}), 880(\mathrm{w}), 863(\mathrm{~m})$. NMR data correspond to the reported values. ${ }^{2}$

\subsection{Synthesis of 1-azido-1,2-benziodoxole-3-(1H)-one (6)}

\section{1-Hydroxy-1,2-benziodoxol-3-(1H)-one (12)}

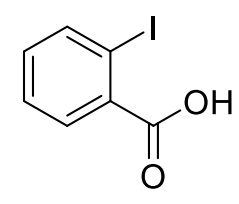

11

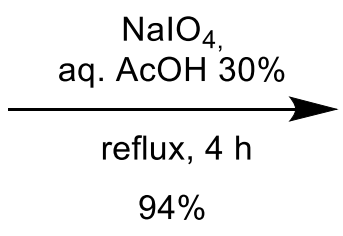

$94 \%$

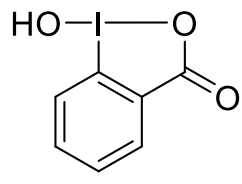

12

Caution: reaction carried out behind a safety shield! Following a reported procedure, ${ }^{3} \mathrm{NaIO}_{4}$ (7.24 g, $33.8 \mathrm{mmol}, 1.00$ equiv) and 2-iodo benzoic acid (11) $(8.00 \mathrm{~g}, 32.2 \mathrm{mmol}, 1.00$ equiv) were suspended in $30 \%$ (v:v) aq $\mathrm{AcOH}(48 \mathrm{~mL})$ under air. The mixture was vigorously stirred and refluxed for $4 \mathrm{~h}$. the reaction mixture was then diluted with cold water $(180 \mathrm{~mL})$ and allowed to cool to room temperature, protecting it from light. The mixture is then filtered and further washed with ice water and cold acetone, air dried in the dark overnight to give the pure compound 12 (8.14 g, $30.4 \mathrm{mmol}, 94 \%)$ as a colorless solid.

${ }^{1} \mathrm{H}$ NMR $\left(400 \mathrm{MHz},\left(\mathrm{CD}_{3}\right)_{2} \mathrm{SO}\right) 8.02(\mathrm{dd}, J=7.7,1.4 \mathrm{~Hz}, 1 \mathrm{H}, \operatorname{Ar} H), 7.97(\mathrm{~m}, 1 \mathrm{H}, \operatorname{Ar} H), 7.85$ $(\mathrm{dd}, J=8.2,0.7 \mathrm{~Hz}, 1 \mathrm{H}, \mathrm{Ar} H), 7.71(\mathrm{td}, J=7.6,1.2 \mathrm{~Hz}, 1 \mathrm{H}, \mathrm{Ar} H) .{ }^{13} \mathbf{C}$ NMR $(100 \mathrm{MHz}$, $\left.\left(\mathrm{CD}_{3}\right)_{2} \mathrm{SO}\right) 167.7,134.5,131.5,131.1,130.4,126.3,120.4$. IR $v_{\max } 3083(\mathrm{w}), 3060(\mathrm{w}), 2867(\mathrm{w})$, 
$2402(\mathrm{w}), 1601(\mathrm{~m}), 1585(\mathrm{~m}), 1564(\mathrm{~m}), 1440(\mathrm{~m}), 1338(\mathrm{~s}), 1302(\mathrm{~m}), 1148(\mathrm{~m}), 1018(\mathrm{w}), 834$ $(\mathrm{m}), 798(\mathrm{w}), 740(\mathrm{~s}), 694(\mathrm{~s}), 674(\mathrm{~m}), 649(\mathrm{~m})$. NMR data correspond to the reported values. ${ }^{3}$

\section{1-Acetoxy-1,2-benziodoxol-3-(1H)-one (13)}<smiles>O=C1OI(O)c2ccccc21</smiles>

12

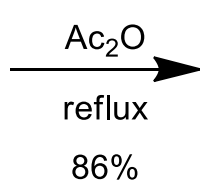

$86 \%$<smiles>CC(=O)OI1OC(=O)c2ccccc21</smiles>

13

Following a reported procedure, ${ }^{4}$ compound $12(3.00 \mathrm{~g}, 11.3 \mathrm{mmol}, 1.00$ equiv) was heated in $\mathrm{Ac}_{2} \mathrm{O}(10 \mathrm{~mL})$ to reflux until the solution turned clear (without suspension). The mixture was then left to cool down and white crystals started to form. The crystallization was continued at -18 ${ }^{\circ} \mathrm{C}$. The crystal were then collected and dried overnight under high vacuum to give compound $\mathbf{1 3}$ (3.06 g, $10.0 \mathrm{mmol}, 86 \%$ ).

${ }^{1} \mathbf{H}$ NMR $\left(400 \mathrm{MHz}, \mathrm{CDCl}_{3}\right) \delta 8.25(\mathrm{dd}, 1 \mathrm{H}, J=7.6,1.4 \mathrm{~Hz}, \mathrm{ArH}), 8.00(\mathrm{dd}, 1 \mathrm{H}, J=8.3,0.5$ $\mathrm{Hz}, \mathrm{ArH}), 7.92(\mathrm{dt}, 1 \mathrm{H}, J=7.0,1.7 \mathrm{~Hz}, \mathrm{ArH}), 7.71(\mathrm{td}, 1 \mathrm{H}, J=7.6,0.9 \mathrm{~Hz}, \operatorname{Ar} H), 2.25(\mathrm{~s}, 3 \mathrm{H}$, $\left.\mathrm{COCH}_{3}\right)$. NMR data correspond to the reported values. ${ }^{4}$

\section{Synthesis of 1-azido-1,2-benziodoxole-3-(1H)-one (6)}<smiles>CC(=O)OI1OC(=O)c2ccccc21</smiles>

13

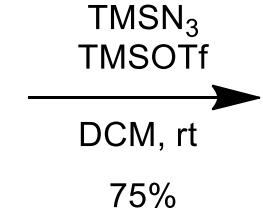

$75 \%$<smiles>N#I1OC(=O)c2ccccc21</smiles>

6

Caution: reaction carried out behind a safety shield! Following a reported procedure, ${ }^{2}$ compound 13 (1.00 g, $3.28 \mathrm{mmol}, 1.00$ equiv) was stirred in dry DCM $(3 \mathrm{~mL})$ then TMSN $_{3}(0.66 \mathrm{~mL}, 4.9$ mmol, 1.5 equiv) was cautiously added. A catalytic amount of TMSOTf ( $3 \mu \mathrm{L}, 0.02 \mathrm{mmol}, 0.005$ equiv) was added last to the mixture which was then stirred for 30 minutes. The reaction mixture was then died in vacuo to give a yellow precipitate, which was washed a few times with hexanes to give compound $\mathbf{6}(0.70 \mathrm{~g}, 2.4 \mathrm{mmol}, 74 \%)$ as a pure pale yellow crystal.

${ }^{1} \mathbf{H}$ NMR $\left(400 \mathrm{MHz}, \mathrm{CDCl}_{3}: \mathrm{CH}_{3} \mathrm{CN} ; 10: 1\right) \delta 8.17(\mathrm{dd}, 1 \mathrm{H}, J=7.5,1.3 \mathrm{~Hz}, \mathrm{ArH}), 7.93(\mathrm{~m}, 2 \mathrm{H}$, $\mathrm{ArH}), 7.70(\mathrm{~m}, 1 \mathrm{H}, \mathrm{ArH}) .{ }^{13} \mathrm{C}$ NMR (101 MHz, $\left.\mathrm{CDCl}_{3}: \mathrm{CH}_{3} \mathrm{CN} ; 10: 1\right) \delta 166.2,134.8,131.8$, 130.4, 125.4, 116.6, 115.4. IR $v_{\max } 2049$ (s), 1639 (s), 1565 (m), 1440 (w), 1347 (w), 1295 (m).The analysis data for the characterization of $\mathbf{6}$ correspond to the ones reported in the literature. $^{2}$ 


\section{Synthesis of starting materials}

\section{General procedure for the synthesis of $\beta$-keto esters.}

Following a slightly modified procedure, ${ }^{5}$ a spatula of sodium tert-butoxide was added to a suspension of $\mathrm{NaH}$ ( 2.2 equiv) in dimethyl carbonate (5.0 equiv). The ketone ( 1 equiv) was then added carefully at $0{ }^{\circ} \mathrm{C}$. The reaction was stirred at room temperature and followed by TLC (using the solvent mixture indicated below for the $\mathrm{R}_{\mathrm{f}}$ value and $\mathrm{UV}$ or $p$-anisaldehyde for visualization). Then $1 \mathrm{M} \mathrm{HCl}$ was added until the $\mathrm{pH}$ of the solution became neutral or slightly acidic. The organic layers were collected and washed with brine $(2 \times 20 \mathrm{~mL})$, dried over $\mathrm{MgSO}_{4}$ and concentrated in vacuum. The crude product was purified by column chromatography, using the solvent indicated for the $\mathbf{R}_{\mathbf{f}}$ value.

\section{Methyl 5-methoxy-1-oxo-2,3-dihydro-1H-indene-2-carboxylate (1a)}

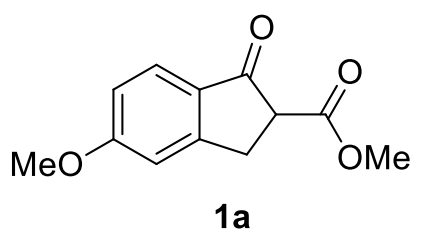

$\left(\mathrm{dd}, 1 \mathrm{H}, J=17.3,3.9 \mathrm{~Hz}, \mathrm{CH}_{2}\right), 3.31\left(\mathrm{dd}, 1 \mathrm{H}, J=17.3,8.2 \mathrm{~Hz}, \mathrm{CH}_{2}\right) .{ }^{13} \mathbf{C}$ NMR $(101 \mathrm{MHz}$, $\left.\mathrm{CDCl}_{3}\right) \delta 197.5,169.9,165.9,156.8,128.4,126.4,116.0,109.6,55.8,53.4,52.8,30.3$. NMR data correspond to the reported values. ${ }^{6}$

\section{Methyl 1-oxo-2,3-dihydro-1H-indene-2-carboxylate (1b)}

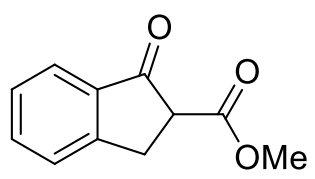

$1 b$

Starting from 1-indanone (0.80 g, $6.0 \mathrm{mmol})$, methyl 1-oxo-2,3-dihydro$1 \mathrm{H}$-indene-2-carboxylate (1b) $(0.77 \mathrm{~g}, 3.0 \mathrm{mmol}, 73 \%$ yield) was obtained as a yellow solid. Rf 0.2 (pentane:ethyl acetate 10:1). ${ }^{\mathbf{1}} \mathbf{H}$ NMR $\left(400 \mathrm{MHz}, \mathrm{CDCl}_{3}\right) \delta 7.78(\mathrm{~d}, 1 \mathrm{H}, J=7.7 \mathrm{~Hz}, \mathrm{ArH}), 7.63(\mathrm{dt}, 1 \mathrm{H}, J=$ 4.0, 8.0 Hz, ArH), $7.50(\mathrm{dd}, 1 \mathrm{H}, J=7.7,0.8 \mathrm{~Hz}, \operatorname{ArH}), 7.40(\mathrm{t}, 1 \mathrm{H}, J=$ $8.0 \mathrm{~Hz}, \mathrm{ArH}), 3.79$ (s, $3 \mathrm{H}), 3.74(\mathrm{dd}, 1 \mathrm{H}, J=8.3,4.1 \mathrm{~Hz}, \mathrm{CH}), 3.57$ (dd, $\left.1 \mathrm{H}, J=17.3,4.0 \mathrm{~Hz}, \mathrm{CH}_{2}\right), 3.38\left(\mathrm{dd}, 1 \mathrm{H}, J=17.3,8.3 \mathrm{~Hz}, \mathrm{CH}_{2}\right) .{ }^{13} \mathbf{C} \mathbf{N M R}\left(101 \mathrm{MHz}, \mathrm{CDCl}_{3}\right)$ $\delta$ 199.4, 169.6, 153.6, 135.5, 135.2, 127.9, 126.6, 124.8, 53.2, 52.8, 30.3. IR $v_{\max } 2954(\mathrm{w}), 1743$ (m), $1711(\mathrm{~s}), 1654(\mathrm{w}), 1608(\mathrm{w}), 1572(\mathrm{w}), 1462(\mathrm{w}), 1439(\mathrm{~m}), 1373(\mathrm{w}), 1329(\mathrm{w}), 1295(\mathrm{w})$, 1261 (m), 1210 (m), 1155 (m), 1125 (w), 1093 (w), 1040 (w), 1020 (w), 990 (m), 962 (w), 915 $(\mathrm{w}), 850(\mathrm{w})$. NMR data correspond to the reported values. ${ }^{7}$

\section{5-Fluoro-1-oxo-2,3-dihydro-1H-indene-2-carboxylate (1c)}

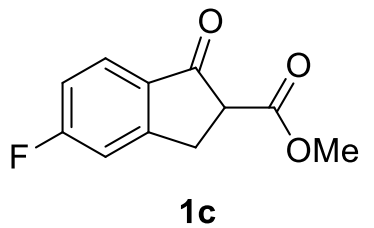

Starting from 5-fluoro-2,3-dihydro- $1 H$-inden-1-one $(0.70 \mathrm{~g}, 4.7 \mathrm{mmol})$, methyl 5-fluoro-1-oxo-2,3-dihydro- $1 H$-indene-2-carboxylate (1c) $(0.80$ g, 4.0 mmol, $85 \%$ yield) was obtained as an orangeish solid. $\mathbf{R}_{\mathbf{f}}: 0.3$ (pentane:EtOAc 10:2). Mp: 55.9-60.7 ${ }^{\circ}$. ${ }^{1} \mathbf{H}$ NMR (400 $\mathrm{MHz}, \mathrm{CDCl}_{3}$ ) ratio ketone/enol 8/1: ketone $\delta 7.78(\mathrm{dd}, 1 \mathrm{H}, J=8.5,5.3 \mathrm{~Hz}, \mathrm{CHAr})$, 7.17 (dd, $1 \mathrm{H}, J=8.3,0.7 \mathrm{~Hz}$, , CHAr), 7.10 (td, $1 \mathrm{H}, J=8.6,1.9 \mathrm{~Hz}$, , CHAr), 3.80 (s, $3 \mathrm{H}, \mathrm{OMe}$ ), 3.77 (dd, $1 \mathrm{H}, J=8.3,4.0 \mathrm{~Hz}, \mathrm{CH}), 3.57$ (dd, $1 \mathrm{H}, J=17.5,4.0 \mathrm{~Hz}$, 
$\left.\mathrm{CH}_{2}\right), 3.36\left(\mathrm{dd}, 1 \mathrm{H}, J=17.5,8.2 \mathrm{~Hz}, \mathrm{CH}_{2}\right)$; enol $\delta 10.41(\mathrm{~m}, 1 \mathrm{H}, \mathrm{OH}), 7.61(\mathrm{dd}, 1 \mathrm{H}, J=8.4$, $5.2 \mathrm{~Hz}, \mathrm{CHAr}), 7.12(\mathrm{td}, 2 \mathrm{H}, J=8.6,1.9 \mathrm{~Hz}, \mathrm{CHAr}), 3.87(\mathrm{~s}, 3 \mathrm{H}, \mathrm{OMe}), 3.53\left(\mathrm{~s}, 2 \mathrm{H}, \mathrm{CH}_{2}\right) .{ }^{13} \mathrm{C}$ NMR (101 MHz, $\left.\mathrm{CDCl}_{3}\right)$ ketone: $\delta$ 197.4, 169.3, $167.6\left(\mathrm{~d}, J_{C-F}=258 \mathrm{~Hz}\right), 156.6\left(\mathrm{~d}, J_{C-F}=11\right.$ $\mathrm{Hz}), 131.6\left(\mathrm{~d}, J_{C-F}=1 \mathrm{~Hz}\right), 127.1\left(\mathrm{~d}, J_{C-F}=11 \mathrm{~Hz}\right), 116.3\left(\mathrm{~d}, J_{C-F}=23 \mathrm{~Hz}\right), 113.3\left(\mathrm{~d}, J_{C-F}=22\right.$ Hz), 53.4, 52.9, 30.1. IR $v_{\max } 3075$ (w), 3066 (w), 2955 (w), 1745 (s), 1716 (s), 1658 (w), 1618 (m), $1596(\mathrm{~m}), 1483(\mathrm{w}), 1449(\mathrm{w}), 1437(\mathrm{w}), 1370(\mathrm{w}), 1331(\mathrm{~m}), 1303(\mathrm{w}), 1252(\mathrm{~s}), 1230(\mathrm{~m})$, $1197(\mathrm{~m}), 1162(\mathrm{~m}), 1134(\mathrm{w}), 1087(\mathrm{~m}), 1018(\mathrm{w}), 990(\mathrm{w}), 963(\mathrm{w}), 937(\mathrm{w}), 874(\mathrm{w}), 861(\mathrm{w})$, $820(\mathrm{w}), 782(\mathrm{w}), 739$ (w). HRMS (ESI) calcd for $\mathrm{C}_{11} \mathrm{FH}_{10} \mathrm{O}_{3}{ }^{+}[\mathrm{M}+\mathrm{H}]^{+}$209.0608; found 209.0607.

\section{Methyl 6-chloro-1-oxo-2,3-dihydro-1H-indene-2-carboxylate (1d)}

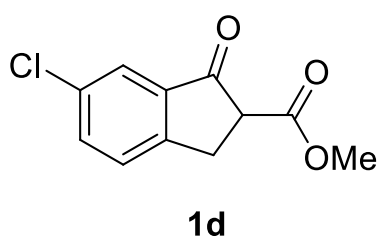

Starting from 6-chloro-2,3-dihydro-1H-inden-1-one $(1.0 \mathrm{~g}, 6.0$ mmol), methyl 6-chloro-1-oxo-2,3-dihydro-1H-indene-2-carboxylate (1d) $(0.51 \mathrm{~g}, 2.3 \mathrm{mmol}, 38 \%$ yield $)$ was obtained as an orange solid. Rf: 0.3 (pentane:EtOAc 10:1). Mp: $119.4-124-6^{\circ} \mathrm{C} .{ }^{\mathbf{1}} \mathbf{H}$ NMR (400 $\left.\mathrm{MHz}, \mathrm{CDCl}_{3}\right)$ ratio ketone/enol 0.7/ 1: ketone $\delta 7.74(\mathrm{~d}, 1 \mathrm{H}, J=1.7$ $\mathrm{Hz}, \mathrm{CHAr}$ ), 7.59 (dd, $1 \mathrm{H}, J=8.2,1.9 \mathrm{~Hz}, \mathrm{CHAr}), 7.45$ (d, $1 \mathrm{H}, J=$ 8.2 Hz, CHAr), 3.80 (s, $3 \mathrm{H}, \mathrm{OMe}$ ), 3.78 (dd, $1 \mathrm{H}, J=8.4,4.1 \mathrm{~Hz}, \mathrm{CH}$ ), 3.54 (dd, $1 \mathrm{H}, J=17.4$, $\left.4.0 \mathrm{~Hz}, \mathrm{CH}_{2}\right), 3.35\left(\mathrm{dd}, 1 \mathrm{H}, J=17.4,8.2 \mathrm{~Hz}, \mathrm{CH}_{2}\right)$; enol $\delta 10.26(\mathrm{~m}, 1 \mathrm{H}, \mathrm{OH}), 7.62(\mathrm{~s}, 1 \mathrm{H}$, CHAr), 7.39 (s, $2 \mathrm{H}, \mathrm{CHAr}), 3.86$ (s, $3 \mathrm{H}, \mathrm{OMe}$ ), 3.50 (s, $2 \mathrm{H}, \mathrm{CH}_{2}$ ). ${ }^{\mathbf{1}} \mathbf{C}$ NMR ketone: $\delta$ 198.1, $169.1,151.6,138.6,135.5,133.1,127.8,124.5,53.6,53.0,29.9$. IR $v_{\max } 3372(w), 3353(w)$, 3322 (w), 3307 (w), 3297 (w), 3285 (w), 3257 (w), 2953 (w), 1746 (w), 1719 (w), 1665 (s), 1619 (m), $1618(\mathrm{~m}), 1596(\mathrm{~m}), 1572(\mathrm{~s}), 1445(\mathrm{~s}), 1395(\mathrm{w}), 1353(\mathrm{~m}), 1307(\mathrm{w}), 1258(\mathrm{~s}), 1239(\mathrm{~s})$, 1211 (s), 1195 (m), $1164(\mathrm{~m}), 1129$ (s), 1106 (m), 1068 (w), 1004 (w), $911(\mathrm{w}), 899(\mathrm{w}), 885(\mathrm{w})$, $805(\mathrm{w}), 775(\mathrm{~m}), 733(\mathrm{w})$. HRMS (ESI) calcd for $\mathrm{C}_{11} \mathrm{H}_{9} \mathrm{ClNaO}_{3}{ }^{+}[\mathrm{M}+\mathrm{Na}]^{+}$247.0132; found 247.0132 .

\section{Tert-butyl 1-oxo-2,3-dihydro-1H-indene-2-carboxylate (1e)}

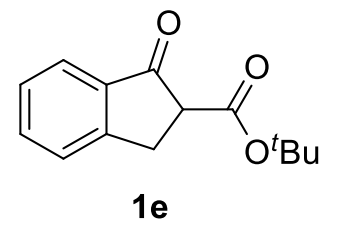

Following a reported procedure, ${ }^{8}$ starting from 1-indanone $(2.0 \mathrm{~g}, 15$ mmol), tert-butyl 1-oxo-2,3-dihydro- $1 H$-indene-2-carboxylate (1e) (1.63 g, 7.02 mmol, $46 \%$ yield) was obtained as a purple oil. $\quad \mathbf{R}_{\mathbf{f}}: 0.4$ (pentane:EtOAc 8:2). ${ }^{1} \mathbf{H}$ NMR $\left(400 \mathrm{MHz}, \mathrm{CDCl}_{3}\right) \delta 7.71(\mathrm{~d}, 1 \mathrm{H}, J=7.7$ $\mathrm{Hz}, \operatorname{Ar} H), 7.57(\mathrm{t}, 1 \mathrm{H}, J=8 \mathrm{~Hz}, \operatorname{Ar} H), 7.45(\mathrm{~d}, 1 \mathrm{H}, J=7.4 \mathrm{~Hz}, \operatorname{Ar} H), 7.34$ $(\mathrm{t}, 1 \mathrm{H}, J=7.1 \mathrm{~Hz}), 3.58(\mathrm{dd}, 1 \mathrm{H}, J=8.2,4.0 \mathrm{~Hz}, \mathrm{CH}), 3.46(\mathrm{dd}, 1 \mathrm{H}, J=$ 8.2, $\left.4.0 \mathrm{~Hz}, \mathrm{CH}_{2}\right), 3.29\left(\mathrm{dd}, 1 \mathrm{H}, J=17.2,8.2 \mathrm{~Hz}, \mathrm{CH}_{2}\right), 1.46\left(\mathrm{~s}, 9 \mathrm{H},\left(\mathrm{CH}_{3}\right)_{3}\right) .{ }^{\mathbf{1 3}} \mathbf{C ~ N M R}(101$ $\left.\mathrm{MHz}, \mathrm{CDCl}_{3}\right) \delta 200.4,168.7,154.1,135.9,135.6,128.1,126.9,124.9,82.4,54.8,30.7,28.4($ 3C). IR $v_{\max } 2979(\mathrm{w}), 2933(\mathrm{w}), 1734$ (s), 1733 (s), 1713 (s), 1645 (w), $1627(\mathrm{w}), 1608(\mathrm{w})$, $1595(\mathrm{w}), 1574(\mathrm{w}), 1476(\mathrm{w}), 1464(\mathrm{w}), 1408(\mathrm{w}), 1393(\mathrm{w}), 1382(\mathrm{w}), 1369(\mathrm{~m}), 1329(\mathrm{w})$, 1298 (w), $1291(\mathrm{w}), 1270(\mathrm{w}), 1254(\mathrm{w}), 1226(\mathrm{w}), 1211(\mathrm{w}), 1149(\mathrm{~s}), 1131(\mathrm{~m}), 1092(\mathrm{w}), 1022$ (w), $1014(\mathrm{w}), 989(\mathrm{w}), 845(\mathrm{w}), 773(\mathrm{w}), 759(\mathrm{w}), 740(\mathrm{~m}), 723(\mathrm{~m}), 680(\mathrm{w}), 669(\mathrm{w}), 642(\mathrm{w})$, $630(\mathrm{w})$. NMR data correspond to the reported values. ${ }^{8}$

\section{Adamantan-1-yl 1-oxo-2,3-dihydro-1H-indene-2-carboxylate (1f)}

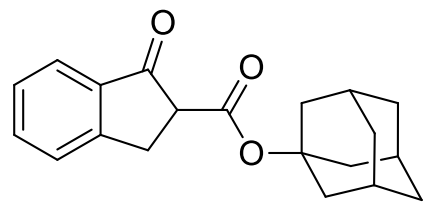

Following a reported procedure, ${ }^{9}$ starting from methyl 1-oxo-2,3dihydro- $1 H$-indene-2-carboxylate (1.0 g, $5.0 \mathrm{mmol}, 1.0 \mathrm{gequiv})$, adamantan-1-yl 1-oxo-2,3-dihydro-1H-indene-2-carboxylate

(1f) 
(788 mg, $2.54 \mathrm{mmol}, 48 \%$ ) was obtained as a pink powder. Rf: 0.6 (pentane:EtOAc 4:1). ${ }^{1} \mathbf{H}$ NMR $\left(400 \mathrm{MHz}, \mathrm{CDCl}_{3}\right) \delta 7.74(\mathrm{~d}, 1 \mathrm{H}, J=7.6 \mathrm{~Hz}, \mathrm{ArH}), 7.58(\mathrm{t}, 1 \mathrm{H}, J=6.9 \mathrm{~Hz}, \operatorname{ArH}), 7.47$ $(\mathrm{d}, 1 \mathrm{H}, J=7.6 \mathrm{~Hz}, \mathrm{ArH}), 7.36(\mathrm{t}, 1 \mathrm{H}, J=8,12 \mathrm{~Hz}, \mathrm{ArH}), 3.59$ (dd, $1 \mathrm{H}, J=8.2,3.9 \mathrm{~Hz}, \mathrm{CH}$ ), $3.47\left(\mathrm{~m}, 1 \mathrm{H}, \mathrm{CH}_{2}\right), 3.31\left(\mathrm{dd}, 1 \mathrm{H}, J=17.2,8.2 \mathrm{~Hz}, \mathrm{CH}_{2}\right), 2.13\left(\mathrm{~m}, 12 \mathrm{H}, \mathrm{CH}_{2}\right), 1.64(\mathrm{~m}, 3 \mathrm{H}$, $\mathrm{CH}) .{ }^{13} \mathrm{C}$ NMR $\left(101 \mathrm{MHz}, \mathrm{CDCl}_{3}\right) \delta 200.1,168.0,153.7,135.5,135.2,127.7,126.5,124.6,82.2$, 54.6, 41.3 (3C), 36.2 (3C), 30.9 (3C), 30.4. IR $v_{\max } 2913$ (s), 2854 (w), 1712 (s), 1640 (w), 1599 (w), $1571(\mathrm{w}), 1519(\mathrm{w}), 1495(\mathrm{w}), 1460(\mathrm{w}), 1383(\mathrm{w}), 1351(\mathrm{w}), 1330(\mathrm{w}), 1291(\mathrm{w}), 1259(\mathrm{~m})$, 1210 (m), 1189 (w), 1159 (w), 1130 (w), 1095 (w), 1056 (m), 1017 (w), 990 (w), 968 (w), 937 (w), $910(\mathrm{w}), 862(\mathrm{w}), 861(\mathrm{w}), 816(\mathrm{w})$. NMR data correspond to the reported values, except the peak at 30.9 in the ${ }^{13} \mathrm{C}$ spectrum (reported as 32.9$){ }^{9}$

\section{N-(4-fluorophenyl)-1-oxo-2,3-dihydro-1H-indene-2-carboxamide (1g)}

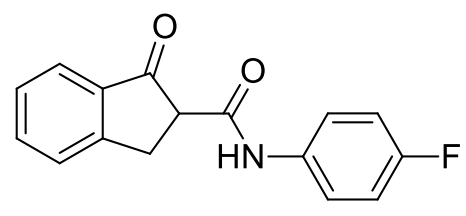

19

Following a slightly modified procedure, ${ }^{10}$ Starting from methyl 1oxo-2,3-dihydro-1H-indene-2-carboxylate (1.0 g, $5.0 \mathrm{mmol}, 1.0$ equiv), $\quad \mathrm{N}$-(4-fluorophenyl)-1-oxo-2,3-dihydro-1H-indene-2carboxamide (1g) (515 mg, $1.91 \mathrm{mmol}, 36 \%)$ was obtained as a pink powder. Rf: 0.63 (pentane:EtOAc 1:1). ${ }^{\mathbf{1}} \mathbf{H}$ NMR $(400 \mathrm{MHz}$, $\left.\mathrm{CDCl}_{3}\right) \delta 9.09(\mathrm{~s}, 1 \mathrm{H}, \mathrm{NH}), 7.73(\mathrm{~d}, 1 \mathrm{H}, J=7.7 \mathrm{~Hz}, \mathrm{ArH}), 7.60$ $(\mathrm{td}, 1 \mathrm{H}, J=7.6,1.1 \mathrm{~Hz}, \operatorname{Ar} H), 7.49(\mathrm{~m}, 3 \mathrm{H}, \mathrm{ArH}), 7.35$ (t, $1 \mathrm{H}, J$ $=8 \mathrm{~Hz}, \operatorname{ArH}), 6.96(\mathrm{~m}, 2 \mathrm{H}, \operatorname{Ar} H), 3.79(\mathrm{dd}, 1 \mathrm{H}, J=17.7,4.1 \mathrm{~Hz}, \mathrm{CH}), 3.67(\mathrm{dd}, 1 \mathrm{H}, J=8.3$, $\left.4.1 \mathrm{~Hz}, \mathrm{CH}_{2}\right), 3.38\left(\mathrm{dd}, 1 \mathrm{H}, J=17.8,8.3 \mathrm{~Hz}, \mathrm{CH}_{2}\right) .{ }^{\mathbf{1 3}} \mathbf{C ~ N M R}\left(101 \mathrm{MHz}, \mathrm{CDCl}_{3}\right) \delta 203.6,164.4$, $159.4\left(\mathrm{~d}, J_{C-F}=244 \mathrm{~Hz}\right), 154.2,136.2,135.2,133.8\left(\mathrm{~d}, J_{C-F}=2 \mathrm{~Hz}\right), 127.9,126.8,124.6,121.7$ $\left(\mathrm{d}, J_{C-F}=8 \mathrm{~Hz}, 2 \mathrm{C}\right), 115.7\left(\mathrm{~d}, J_{C-F}=23 \mathrm{~Hz}, 2 \mathrm{C}\right), 53.2$, 28.5. IR $v_{\max } 3339(\mathrm{w}), 2980(\mathrm{w}), 2939$ (w), $2356(\mathrm{w}), 2355(\mathrm{w}), 2343(\mathrm{w}), 1717(\mathrm{~s}), 1651(\mathrm{~m}), 1607(\mathrm{w}), 1530(\mathrm{~s}), 1515$ (s), $1463(\mathrm{w})$, 1408 (m), 1380 (w), 1330 (w), 1276 (m), 1221 (m), 1164 (m), 1099 (w), 1040 (w), 1010 (w), 930 (w), 838 (s). HRMS (ESI) calcd for $\mathrm{C}_{16} \mathrm{H}_{13} \mathrm{NO}_{2} \mathrm{~F}^{+}(\mathrm{M}+\mathrm{H}) 270.0930$, found 270.0922 .

\section{Methyl 2-methyl-3-oxo-3-phenylpropanoate (1h)}

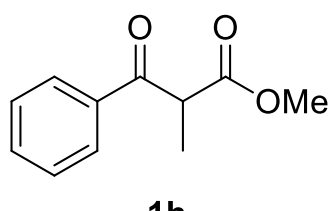

1h

Starting from propiophenone $(0.800 \mathrm{~mL}, 5.96 \mathrm{mmol})$, methyl 2-methyl3-oxo-3-phenylpropanoate (1h) $(0.917 \mathrm{~g}, 4.77 \mathrm{mmol}, 80 \%$ yield) (was obtained as a yellow liquid. Rf: 0.4 (pentane:EtOAc 4:1). ${ }^{\mathbf{1}} \mathbf{H}$ NMR (400 $\left.\mathrm{MHz}_{\mathrm{CDCl}}\right) \delta 7.97(\mathrm{~m}, 2 \mathrm{H}, \mathrm{ArH}), 7.58(\mathrm{~m}, 1 \mathrm{H}, \mathrm{ArH}), 7.47(\mathrm{~m}, 2 \mathrm{H}$, ArH), 4.41 (q, $1 \mathrm{H}, J=7.1 \mathrm{~Hz}, \mathrm{CH}), 3.67$ (s, $3 \mathrm{H}, \mathrm{OMe}), 1.49$ (d, $3 \mathrm{H}, J$ $=7.1 \mathrm{~Hz}, \mathrm{CHMe}) .{ }^{13} \mathbf{C} \mathbf{N M R}\left(101 \mathrm{MHz}, \mathrm{CDCl}_{3}\right) \delta 195.9,171.3,135.8$, 133.6, $128.8(2 \mathrm{C}), 128.6(2 \mathrm{C}), 52.5,48.1,13.9$. NMR data correspond to the reported values. ${ }^{11}$

\section{Methyl 2-methyl-3-oxo-3-(4-(trifluoromethyl)phenyl) propanoate (1i)}<smiles>COC(=O)C(C)C(=O)c1ccc(C(F)(F)F)cc1</smiles>

$1 \mathbf{i}$

Starting from 1-(4-(trifluoromethyl)phenyl)propan-1-one (0.70 g, 3.5 mmol), methyl 2-methyl-3-oxo-3-(4-(trifluoromethyl)phenyl) propanoate (1i) $(0.740 \mathrm{~g}, 2.84 \mathrm{mmol}, 82 \%$ yield) was obtained as a colorless liquid. Rf: 0.3 (pentane:EtOAc 1:1). ${ }^{1} \mathbf{H}$ NMR $(400 \mathrm{MHz}$, $\left.\mathrm{CDCl}_{3}\right) \delta 8.08(\mathrm{~d}, 2 \mathrm{H}, J=8.2 \mathrm{~Hz}, \mathrm{CHAr}), 7.75(\mathrm{~d}, 2 \mathrm{H}, J=8.2 \mathrm{~Hz}$, CHAr), $4.40(\mathrm{q}, 1 \mathrm{H}, J=7.1 \mathrm{~Hz}, \mathrm{CH}), 3.70(\mathrm{~s}, 1 \mathrm{H}, \mathrm{OMe}), 1.52\left(\mathrm{~d}, 2 \mathrm{H}, J=7.1 \mathrm{~Hz}, \mathrm{CH} .{ }^{13} \mathrm{C}\right.$ 
NMR $\left(101 \mathrm{MHz}, \mathrm{CDCl}_{3}\right) \delta 194.8,170.9,138.5,134.8(\mathrm{q}, J=32 \mathrm{~Hz}), 128.9(2 \mathrm{C}), 125.9(\mathrm{q}, J=4$ Hz, 2C), 123.5 (q, J = 273 Hz), 52.7, 48.4, 13.6. IR $v_{\max } 2997$ (w), 2957 (w), 1745 (m), 1696 (m), $1582(\mathrm{w}), 1513(\mathrm{w}), 1456$ (w), $1438(\mathrm{w}), 1411(\mathrm{w}), 1325(\mathrm{~s}), 1223(\mathrm{w}), 1171(\mathrm{~s}), 1132(\mathrm{~s}), 1067$ (s), $1017(\mathrm{w}), 980(\mathrm{w}), 950(\mathrm{w}), 853(\mathrm{~m}), 822(\mathrm{w}), 741(\mathrm{w}), 729$ (w), 714 (w). NMR data correspond to the reported values. ${ }^{11}$

\section{3-(4-Bromophenyl)-2-methyl-3-oxopropanoate (1j)}<smiles>COC(=O)C(C)C(=O)c1ccc(Br)cc1</smiles>

$1 \mathbf{j}$

Starting from 1-(4-bromophenyl)propan-1-one $(0.80 \mathrm{~g}, 6.0 \mathrm{mmol})$, methyl 3-(4-bromophenyl)-2-methyl-3-oxopropanoate (1j) (0.52 g, 1.9 mmol, $41 \%$ yield) was obtained as a colorless liquid. Rf: 0.4 (pentane:EtOAc 1:1). ${ }^{\mathbf{1}} \mathbf{H}$ NMR $\left(400 \mathrm{MHz} \mathrm{CDCl}_{3}\right) \delta 7.84(\mathrm{~m}, 2 \mathrm{H}$, CHAr), 7.62 (d, $2 \mathrm{H}, J=8.4 \mathrm{~Hz}, \mathrm{CHAr}), 4.34$ (q, $1 \mathrm{H}, J=7.0 \mathrm{~Hz}$, $\mathrm{CH}), 3.68(\mathrm{~m}, 3 \mathrm{H}, \mathrm{OMe}), 1.49$ (d, $\left.3 \mathrm{H}, J=7.0 \mathrm{~Hz}, \mathrm{CH}_{3}\right) .{ }^{13} \mathrm{C}$ NMR $\left(101 \mathrm{MHz} \mathrm{CDCl}_{3}\right) \delta 194.7,171.0,134.5,132.2$ (2C), 130.1 (2C), 128.9, 52.7, 48.1, 13.7. NMR data correspond to the reported values. ${ }^{12}$

\section{Methyl 2-methyl-3-oxo-3-(thiophen-2-yl)propanoate (1k)}

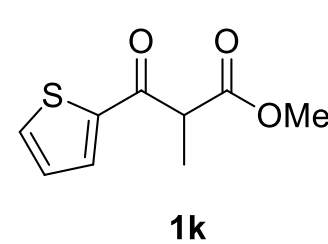

Starting from 1-(thiophen-2-yl)propan-1-one (0.70 g, $4.9 \mathrm{mmol})$, methyl 2-methyl-3-oxo-3-(thiophen-2-yl)propanoate (1k) (0.96 g, 4.8 mmol, 97 $\%$ yield) was obtained as an orange liquid. Rf: 0.3 (pentane:EtOAc 3:2). ${ }^{1} \mathbf{H}$ NMR $\left(400 \mathrm{MHz}, \mathrm{CDCl}_{3}\right) \delta 7.78$ (m, $\left.1 \mathrm{H}, \mathrm{CHAr}\right), 7.69$ (dd, $1 \mathrm{H}, J=$ 4.9, 0.9 Hz, CHAr), 7.15 (m, $1 \mathrm{H}, \mathrm{CHAr}), 4.26$ (q, $1 \mathrm{H}, J=7.1 \mathrm{~Hz}$,

$\mathrm{CH}), 3.71(\mathrm{~s}, 3 \mathrm{H}, \mathrm{OMe}), 1.51\left(\mathrm{~d}, 3 \mathrm{H}, J=7.1 \mathrm{~Hz}, \mathrm{CH}_{3}\right) .{ }^{\mathbf{1 3}} \mathrm{C}$ NMR (101 $\left.\mathrm{MHz}_{2} \mathrm{CDCl}_{3}\right) \delta 188.5,170.9,143.0,134.8,132.9,128.4,52.7,49.3,14.0$. NMR data correspond to the reported values. ${ }^{11}$

\section{Tert-butyl 1-oxo-1,2,3,4-tetrahydronaphthalene-2-carboxylate (15)}

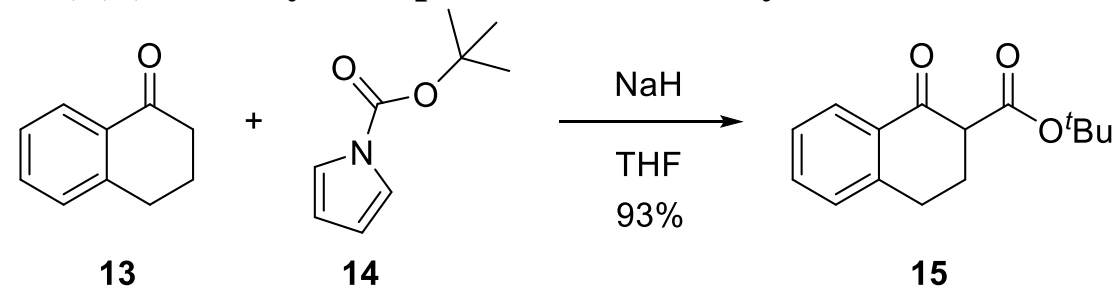

Compound 15 was synthetized following a reported procedure. ${ }^{8} \alpha$-tetralone (13) (1.0 g, 6.8 $\mathrm{mmol})$ in dry THF ( $5 \mathrm{~mL})$ was added to a suspension of $\mathrm{NaH}(0.547 \mathrm{mg}, 13.7 \mathrm{mmol}, 2.00$ equiv, $60 \%$ dispersion in mineral oil) in dry THF $(25 \mathrm{~mL})$ at $\mathrm{rt}$. The solution was warmed to reflux and tert butyl pyrrole-1-carboxylate (14) $(2.29 \mathrm{~g}, 13.7 \mathrm{mmol}, 2.00$ equiv) in dry THF (3 mL) was added dropwise and the solution was stirred at reflux until completion as indicated by TLC. After cooling to $0{ }^{\circ} \mathrm{C}$, the solution was acidified with $1 \mathrm{~N} \mathrm{HCl}$. The solution was extracted with EtOAc $(30 \mathrm{~mL})$ and the organic layer was washed with brine $(30 \mathrm{~mL})$, dried $\left(\mathrm{Na}_{2} \mathrm{SO}_{4}\right)$ and concentrated under reduced pressure. The residue was purified by flash silica gel chromatography $\left(\mathrm{R}_{\mathrm{f}}: 0.3 ; 6: 4\right.$ Pentane/ethylacetate). 


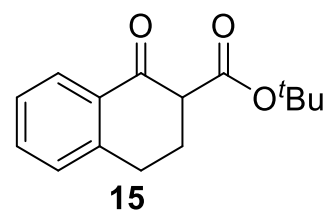

${ }^{1}$ H NMR $\left(400 \mathrm{MHz}, \mathrm{CDCl}_{3}\right)$ enol $\delta 12.65(\mathrm{~s}, 1 \mathrm{H}$, enol H), $7.81(\mathrm{dd}, J=$ 7.5, $1.6 \mathrm{~Hz}, 1 \mathrm{H}, \mathrm{Ar} \mathrm{H}), 7.31$ (td, $J=7.2,1.7 \mathrm{~Hz}, 2 \mathrm{H}, \mathrm{Ar} \mathrm{H}), 7.18$ (ddd, $J=$ 6.6, 1.9, $1.0 \mathrm{~Hz}, 1 \mathrm{H}, \mathrm{Ar} \mathrm{H}), 2.81$ (dd, $\left.J=8.8,6.7 \mathrm{~Hz}, 2 \mathrm{H}, \mathrm{CH}_{2}\right), 2.56-$ $2.51\left(\mathrm{~m}, 2 \mathrm{H}, \mathrm{CH}_{2}\right), 1.57(\mathrm{~s}, 9 \mathrm{H}, t \mathrm{Bu})$. ketone: $\delta 8.07(\mathrm{dd}, J=7.9,1.4 \mathrm{~Hz}, 1$ $\mathrm{H}, \mathrm{Ar} \mathrm{H}), 7.51(\mathrm{td}, J=7.5,1.5 \mathrm{~Hz}, 1 \mathrm{H}, \mathrm{Ar} \mathrm{H}), 7.31(\mathrm{td}, J=7.2,1.7 \mathrm{~Hz}, 1$ $\mathrm{H}, \mathrm{Ar} \mathrm{H}), 7.28(\mathrm{~m}, 1 \mathrm{H}, \mathrm{Ar} \mathrm{H}), 3.52(\mathrm{dd}, J=9.9,4.7 \mathrm{~Hz}, 1 \mathrm{H}, \mathrm{CH}), 3.13-2.95\left(\mathrm{~m}, 2 \mathrm{H}, \mathrm{CH}_{2}\right)$, $2.51-2.44\left(\mathrm{~m}, 1 \mathrm{H}, \mathrm{CH}_{2}\right), 2.36$ (ddt, $\left.J=13.5,6.2,4.8 \mathrm{~Hz}, 1 \mathrm{H}, \mathrm{CH}_{2}\right), 1.50(\mathrm{~s}, 9 \mathrm{H}, t \mathrm{Bu})$.

NMR data correspond to the reported values. ${ }^{13}$

\section{Methyl 2-oxocyclopentane-1-carboxylate (17)}

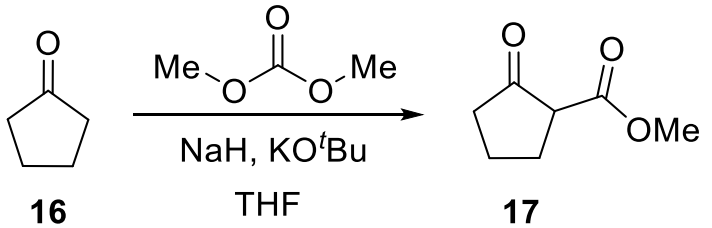

Compound 17 was synthesized following a reported procedure. ${ }^{5}$ In a $50 \mathrm{~mL}$ flask, $\mathrm{NaH}(1.05 \mathrm{~g}$, $26.2 \mathrm{mmol}, 2.2$ equiv) was suspended in THF $(12 \mathrm{~mL})$. Dimethyl carbonate $(5.00 \mathrm{~mL}, 59.4$ mmol, 5.00 equiv) was added to the mixture together with a catalytic amount of potassium tertbutoxide (67 mg, $0.59 \mathrm{mmol}, 0.050$ equiv). The solution was stirred for 1-2 minutes and then cyclopentanone (16) $(1.0 \mathrm{~g}, 12 \mathrm{mmol})$ was added dropwise. The reaction was stirred at room temperature for $5 \mathrm{~h}$. Water was then added carefully to quench the reaction. The aqueous layer was washed with $\mathrm{Et}_{2} \mathrm{O}(2 \times 50 \mathrm{~mL})$ and ethylacetate $(2 \times 50 \mathrm{~mL})$. The organic layers were collected and washed with brine $(2 \times 50 \mathrm{~mL})$. The solvent was evaporated under reduced pressure and the crude product was purified by flash chromatography (95:5 Pentane/ethylacetate) to give compound 17 (0.318 g, $2.24 \mathrm{mmol}, 19 \%)$.

${ }^{1} \mathbf{H}$ NMR $\left(400 \mathrm{MHz}, \mathrm{CDCl}_{3}\right) \delta 3.73$ (s, $\left.3 \mathrm{H}, \mathrm{OMe}\right), 3.16(\mathrm{t}, J=9.0 \mathrm{~Hz}, 1 \mathrm{H}, \mathrm{CH}), 2.35-2.24(\mathrm{~m}$, $\left.4 \mathrm{H}, \mathrm{CH}_{2}\right), 2.18-2.07\left(\mathrm{~m}, 1 \mathrm{H}, \mathrm{CH}_{2}\right), 1.93-1.78\left(\mathrm{~m}, 1 \mathrm{H}, \mathrm{CH}_{2}\right)$.

NMR data correspond to the reported values. ${ }^{14}$

\section{Synthesis of silyl enol ethers}

Silyl enol ethers 4a-h are synthesized following reported procedures.

\section{((1H-Inden-3-yl)oxy)trimethylsilane (4a)}

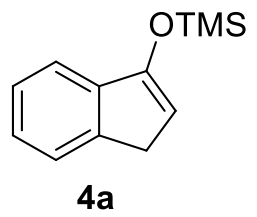

Following a reported procedure, ${ }^{15}$ starting from 1-indanone $(0.50 \mathrm{~g}, 3.8 \mathrm{mmol})$, ((1H-inden-3-yl)oxy)trimethylsilane (4a) $(0.69 \mathrm{~g}, 3.4 \mathrm{mmol}, 90 \%$ yield $)$ was obtained as a yellow liquid. ${ }^{1} \mathbf{H}$ NMR $\delta 7.38(\mathrm{~m}, 2 \mathrm{H}, \mathrm{CHAr}), 7.29(\mathrm{~m}, 1 \mathrm{H}$, CHAr), 7.21 (m, 1 H, CHAr), $5.43(\mathrm{t}, 1 \mathrm{H}, J=2.4 \mathrm{~Hz}, \mathrm{CH}), 3.27(\mathrm{~d}, 2 \mathrm{H}, J=2.4$ $\left.\mathrm{Hz}, \mathrm{CH}_{2}\right), 0.31(\mathrm{~m}, 9 \mathrm{H}, \mathrm{TMS})$. NMR data correspond to the reported values. ${ }^{16}$

\section{((1H-Inden-3-yl)oxy)triethylsilane (4b)}

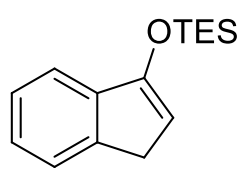

4b 
$=2.2 \mathrm{~Hz}, \mathrm{CH}), 3.27\left(\mathrm{~d}, 2 \mathrm{H}, J=2.1 \mathrm{~Hz}, \mathrm{CH}_{2}\right), 1.03\left(\mathrm{t}, 9 \mathrm{H}, J=8.0 \mathrm{~Hz}, \mathrm{CH}_{2}\left(\mathrm{CH}_{3}\right)_{3}\right), 0.79(\mathrm{q}, 6 \mathrm{H}$, $\left.J=7.7 \mathrm{~Hz}, \mathrm{CH}_{2}\left(\mathrm{CH}_{3}\right)_{3}\right)$. NMR data correspond to the reported values. ${ }^{17}$

\section{((1H-Inden-3-yl)oxy)triisopropylsilane (4c)}

Starting from 1-indanone $(1.0 \mathrm{~g}, 7.6 \mathrm{mmol}), \quad((1 H$-inden-3-
yl)oxy)triisopropylsilane $(\mathbf{4 b})(1.05 \mathrm{~g}, 3.64 \mathrm{mmol}, 48 \%$ yield $)$ was obtained as a
colorless liquid. ${ }^{\text {H NMR }} \delta 7.42(\mathrm{dd}, 2 \mathrm{H}, J=19.5,7.4 \mathrm{~Hz}, \mathrm{CHAr}), 7.31(\mathrm{t}, 1 \mathrm{H}$,
$J=7.3 \mathrm{~Hz}, \mathrm{CHAr}), 7.22(\mathrm{t}, 1 \mathrm{H}, J=7.3 \mathrm{~Hz}, \mathrm{CHAr}), 5.42(\mathrm{t}, 1 \mathrm{H}, J=2.1 \mathrm{~Hz}$,
$\mathrm{CH}), 3.27\left(\mathrm{~d}, 2 \mathrm{H}, J=1.9 \mathrm{~Hz}, \mathrm{CH}_{2}\right), 1.34(\mathrm{~m}, 3 \mathrm{H}, \mathrm{TIPSCH}), 1.15(\mathrm{~d}, 18 \mathrm{H}, J=$
$7.4 \mathrm{~Hz}$, TIPSC $\mathrm{H}_{3}$ ). NMR data correspond to the reported values. ${ }^{18}$

\section{((3,4-Dihydronaphthalen-1-yl)oxy)trimethylsilane (4d)}

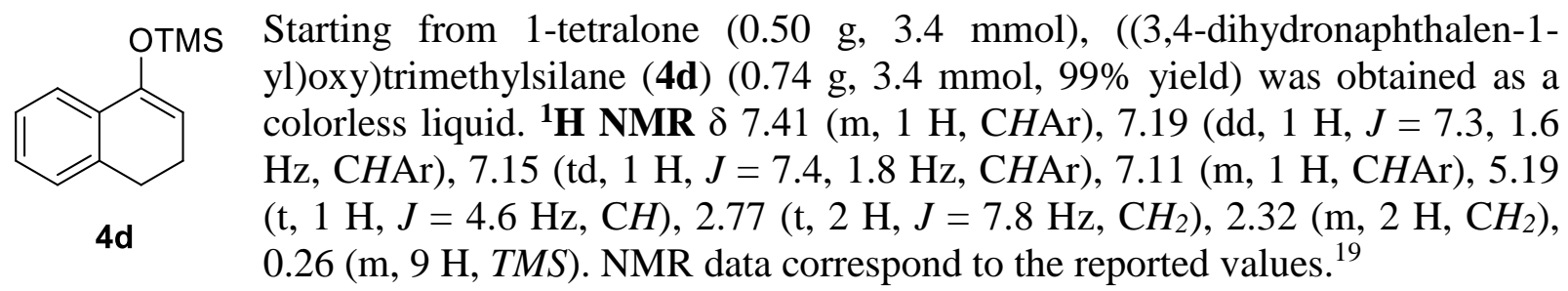

Trimethyl((2-methyl-1H-inden-3-yl)oxy)silane (4e)

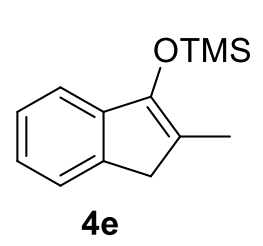

Starting from 2-methyl-2,3-dihydro- $1 H$-inden-1-one $(0.470 \mathrm{ml}, 3.42 \mathrm{mmol})$, trimethyl((2-methyl-1H-inden-3-yl)oxy)silane (4e) $(0.62 \mathrm{~g}, 3.1 \mathrm{mmol}, 90 \%$ yield) was obtained as a colorless liquid. ${ }^{1} \mathbf{H}$ NMR $\delta 7.31(\mathrm{~d}, 1 \mathrm{H}, J=7.3 \mathrm{~Hz}$, CHAr), 7.23 (d, $1 \mathrm{H}, J=7.3 \mathrm{~Hz}, \mathrm{CHAr}), 7.18(\mathrm{~m}, 1 \mathrm{H}, \mathrm{CHAr}), 7.12$ (td, $1 \mathrm{H}, J$ $=7.3,1.2 \mathrm{~Hz}, \mathrm{CHAr}), 3.19\left(\mathrm{~s}, 2 \mathrm{H}, \mathrm{CH}_{2}\right), 1.97\left(\mathrm{~s}, 3 \mathrm{H}, \mathrm{CH}_{3}\right), 0.27(\mathrm{~s}, 9 \mathrm{H}$, $T M S)$. NMR data correspond to the reported values. ${ }^{20}$

\section{Trimethyl((2-methyl-3,4-dihydronaphthalen-1-yl)oxy)silane (4f)}

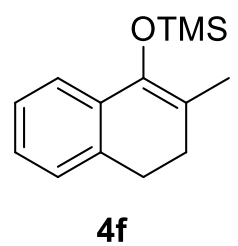

Starting from 2-methyl-3,4-dihydronaphthalen-1(2H)-one $(0.50 \mathrm{~g}, 3.1 \mathrm{mmol})$, trimethyl((2-methyl-3,4-dihydronaphthalen-1-yl)oxy)silane (4f) $(0.38 \mathrm{~g}, 1.6$ mmol, 52\% yield) was obtained as a colorless liquid. ${ }^{1} \mathbf{H}$ NMR $\delta 7.31(\mathrm{~d}, 1 \mathrm{H}$, $J=7.5 \mathrm{~Hz}, \mathrm{CHAr}$ ), $7.16(\mathrm{~m}, 1 \mathrm{H}, \mathrm{CHAr}), 7.09$ (m, $2 \mathrm{H}, \mathrm{CHAr}), 2.74(\mathrm{t}, 2 \mathrm{H}, J$ $\left.=7.8 \mathrm{~Hz}, \mathrm{CH}_{2}\right), 2.26\left(\mathrm{t}, 2 \mathrm{H}, J=8.0 \mathrm{~Hz}, \mathrm{CH}_{2}\right), 1.82\left(\mathrm{~s}, 3 \mathrm{H}, \mathrm{CH}_{3}\right), 0.21(\mathrm{~s}, 9 \mathrm{H}$, $T M S)$. NMR data correspond to the reported values. ${ }^{20}$

\section{Trimethyl((1-phenylvinyl)oxy)silane (4g)}<smiles>C=C(O[Na])c1ccccc1</smiles>

$4 \mathrm{~g}$

Starting from acetophenone $(0.58 \mathrm{~g}, \quad 4.8 \mathrm{mmol})$, trimethyl $((1-$ phenylvinyl)oxy)silane (4g) (0.86 g, $4.5 \mathrm{mmol}, 93 \%$ yield) was obtained as a colorless liquid. ${ }^{1} \mathbf{H}$ NMR $\delta 7.60$ (m, 2 H, CHAr), 7.32 (m, $\left.3 \mathrm{H}, \mathrm{CHAr}\right), 4.92$ (d, $\left.1 \mathrm{H}, J=1.6 \mathrm{~Hz}, \mathrm{CH}_{2}\right), 4.43$ (d, $\left.1 \mathrm{H}, J=1.6 \mathrm{~Hz}, \mathrm{CH}_{2}\right), 0.27$ (s, $9 \mathrm{H}, T M S$ ). NMR data correspond to the reported values. ${ }^{21}$

(Z)-Trimethyl((1-phenylprop-1-en-1-yl)oxy)silane (4h) 


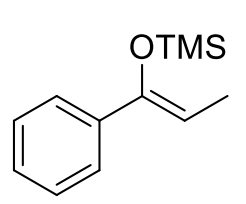

4h

Starting from propiophenone $(0.53 \mathrm{~g}, 4.0 \mathrm{mmol}),(Z)$-trimethyl((1-phenylprop1-en-1-yl)oxy)silane (4h) (1.05 g, $5.10 \mathrm{mmol}, 85 \%$ yield) was obtained as a colorless liquid. ${ }^{1} \mathbf{H}$ NMR $\delta 7.46$ (m, $\left.2 \mathrm{H}, \mathrm{CHAr}\right), 7.29$ (m, $\left.2 \mathrm{H}, \mathrm{CHAr}\right), 7.23$ (d, $1 \mathrm{H}, J=7.1 \mathrm{~Hz}, \mathrm{CHAr}), 5.33$ (q, $1 \mathrm{H}, J=6.9 \mathrm{~Hz}, \mathrm{CH}), 1.74$ (d, $3 \mathrm{H}, J=6.9$ $\left.\mathrm{Hz}, \mathrm{CH}_{3}\right), 0.14$ (s, $\left.9 \mathrm{H}, T M S\right)$. NMR data correspond to the reported values. ${ }^{21}$

\section{General procedure for the synthesis of silyl enol ethers.}

The ketone (1.00 equiv) dissolved in anhydrous THF (0.33 $\mathrm{M}$ in ketone) was added in an ovendried flask sealed with a septum and under $\mathrm{N}_{2}$ atmosphere. The solution was cooled down to $0{ }^{\circ} \mathrm{C}$ and a $2 \mathrm{M}$ solution of NaHMDS (1.22 eq) or freshly prepared LDA (1.05 equiv) was added dropwise. The cold bath is removed and the yellow solution was stirred for 1 hour at room temperature. The reaction was cooled again at $0{ }^{\circ} \mathrm{C}$ and trimethyl silyl chloride (1.20 eq) was added dropwise. The reaction was stirred at room temperature for 5 hours after what the solvent was directly removed under reduced pressure. The resulting oil was purified by plug or by column chromatography on silica using the solvent indicated for the $\mathbf{R}_{\mathbf{f}}$ value.

\section{(Z)-((1-(4-Bromophenyl)prop-1-en-1-yl)oxy)trimethylsilane (4i)}

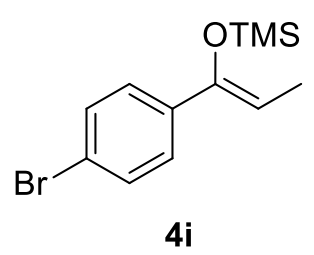

Starting from 1-(4-bromophenyl)propan-1-one (0.50 g, $2.4 \mathrm{mmol}),(Z)-((1-$ (4-bromophenyl)prop-1-en-1-yl)oxy)trimethylsilane (4i) $(0.54 \mathrm{~g}, 1.9 \mathrm{mmol}$, $81 \%$ yield) was obtained as a yellow liquid. Rf: 0.2 (100\% pentane). ${ }^{1} \mathbf{H}$ NMR (400 MHz, $\left.\mathrm{CDCl}_{3}\right) \delta 7.41$ (m, $\left.2 \mathrm{H}, \mathrm{CHAr}\right), 7.33$ (m, $\left.2 \mathrm{H}, \mathrm{CHAr}\right), 5.33$ $(\mathrm{q}, 1 \mathrm{H}, J=6.9 \mathrm{~Hz}, \mathrm{CCH}), 1.73\left(\mathrm{~d}, 3 \mathrm{H}, J=6.9 \mathrm{~Hz}, \mathrm{CH}_{3}\right), 0.14(\mathrm{~m}, 9 \mathrm{H}$, $\left.\left(\mathrm{CH}_{3}\right)_{3}\right) .{ }^{13} \mathrm{C}$ NMR $\left(101 \mathrm{MHz}, \mathrm{CDCl}_{3}\right) \delta 148.9,138.2,131.2(2 \mathrm{C}), 126.8$ (2C), 121.1, 106.1, 11.8, 0.6 (3C). IR $v_{\max } 3061(\mathrm{w}), 3041(\mathrm{w}), 2960(\mathrm{w}), 2914(\mathrm{w}), 2859(\mathrm{w})$, 1652 (m), $1590(\mathrm{w}), 1446(\mathrm{w}), 1397(\mathrm{w}), 1379(\mathrm{w}), 1323(\mathrm{~m}), 1251(\mathrm{~s}), 1181(\mathrm{w}), 1118(\mathrm{w}), 1105$ (w), 1069 (s), 1009 (m), 972 (w), 878 (s), 846 (s), 821 (s), 795 (w), 759 (m), 721 (w), $704(\mathrm{w})$. HRMS (APPI) calcd for $\mathrm{C}_{12} \mathrm{H}_{17}{ }^{79} \mathrm{BrOSi}[\mathrm{M}+]$ 284.0232; found 284.0240.

\section{(Z)-Trimethyl((1-(4-(trifluoromethyl)phenyl)prop-1-en-1-yl)oxy)silane (4j)}

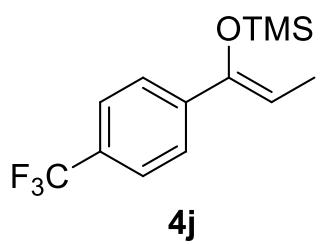

$4 \mathbf{j}$

Starting from 1-(4-(trifluoromethyl)phenyl)propan-1-one (0.50 g, $2.5 \mathrm{mmol})$, (Z)-trimethyl((1-(4-(trifluoromethyl)phenyl)prop-1-en-1-yl)oxy)silane $\quad \mathbf{4} \mathbf{j})$ (0.30 g, $1.2 \mathrm{mmol}, 47 \%$ yield) was obtained as a yellow liquid. $\mathbf{R}_{\mathbf{f}}: 0.2$ (100\% pentane). ${ }^{1} \mathbf{H}$ NMR (400 $\left.\mathrm{MHz}, \mathrm{CDCl}_{3}\right) \delta 7.56(\mathrm{~m}, 4 \mathrm{H}, \mathrm{CHAr}), 5.45$ $(\mathrm{q}, 1 \mathrm{H}, J=6.9 \mathrm{~Hz}, \mathrm{CCH}), 1.77(\mathrm{~d}, 3 \mathrm{H}, J=6.9 \mathrm{~Hz}, \mathrm{CH}), 0.16(\mathrm{~m}, 9 \mathrm{H}$, $\left.\left(\mathrm{CH}_{3}\right)_{3}\right) .{ }^{13} \mathrm{C}$ NMR $\left(101 \mathrm{MHz}, \mathrm{CDCl}_{3}\right) \delta 148.7,142.6,129.2\left(\mathrm{q}, J_{C-F}=32\right.$ $\mathrm{Hz}), 125.2(2 \mathrm{C}), 125.1\left(\mathrm{q}, J_{C-F}=4 \mathrm{~Hz}, 2 \mathrm{C}\right), 124.3(\mathrm{q}, J=272 \mathrm{~Hz}), 107.7,11.8,0.6(3 \mathrm{C}) . \mathbf{I R} v_{\max }$ $3067(w), 3052$ (w), 2962 (w), 2918 (w), 2863 (w), 1651 (w), 1618 (w), 1448 (w), $1411(w)$, 1381 (w), 1290 (w), 1255 (m), 1167 (m), 1124 (s), 1113 (s), 1067 (s), 972 (w), 957 (w), 877 (s), 844 (s), 797 (m), 758 (m), 704 (w). HRMS (APPI) calcd for $\mathrm{C}_{13} \mathrm{H}_{17} \mathrm{~F}_{3} \mathrm{OSi}[\mathrm{M}+]$ 274.1001; found 274.1009 .

\section{(Z)-Trimethyl((1-(thiophen-2-yl)prop-1-en-1-yl)oxy)silane (4k)}




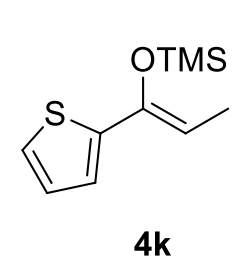

Starting from 1-(thiophen-2-yl)propan-1-one $(0.50 \mathrm{~mL}, 4.0 \mathrm{mmol})(\mathbf{1 k})(\mathrm{Z})$ trimethyl((1-(thiophen-2-yl)prop-1-en-1-yl)oxy)silane (4k) $(0.648 \mathrm{~g}, 3.05 \mathrm{mmol}$, $76 \%$ yield) was obtained as a yellow liquid. Rf: 0.3 (pentane:EtOAc 4:1). ${ }^{1} \mathbf{H}$ NMR $\left(400 \mathrm{MHz}, \mathrm{CDCl}_{3}\right) \delta 7.10(\mathrm{dd}, 1 \mathrm{H}, J=5.0,1.1 \mathrm{~Hz}, \mathrm{C} \underline{H A r}), 7.02(\mathrm{dd}, 1 \mathrm{H}$, $J=3.6,1.0 \mathrm{~Hz}, \mathrm{C} \underline{H} \mathrm{Ar}), 6.93(\mathrm{dd}, 1 \mathrm{H}, J=5.0,3.6 \mathrm{~Hz}, \mathrm{C} \underline{H A r}), 5.32$ (q, $1 \mathrm{H}, J=$ $6.9 \mathrm{~Hz}, \mathrm{C} \underline{H}), 1.71\left(\mathrm{~d}, 3 \mathrm{H}, J=6.9 \mathrm{~Hz}, \mathrm{C} \underline{H_{3}}\right), 0.22(\mathrm{~s}, 9 \mathrm{H}, \underline{T M S}) .{ }^{13} \mathbf{C}$ NMR (101 $\left.\mathrm{MHz}_{2} \mathrm{CDCl}_{3}\right) \delta 144.3,143.1,126.4,123.0,122.0,104.2,11.0,0.0$ (3C). IR $v_{\max } 2961(\mathrm{w}), 2914$ (w), $2860(\mathrm{w}), 1650(\mathrm{w}), 1523(\mathrm{w}), 1437(\mathrm{w}), 1413(\mathrm{w}), 1382(\mathrm{w}), 1357(\mathrm{w}), 1326(\mathrm{~m}), 1312(\mathrm{w})$, 1250 (s), 1215 (w), 1104 (w), 1062 (s), 1039 (m), $960(\mathrm{w}), 938(\mathrm{w}), 912(\mathrm{w}), 868(\mathrm{~s}), 846(\mathrm{~s}), 796$ $(\mathrm{w}), 755(\mathrm{~m}), 738(\mathrm{w}), 705(\mathrm{w})$. HRMS (ESI) calcd for $\mathrm{C}_{10} \mathrm{H}_{17} \mathrm{OSSi}^{+}[\mathrm{M}+\mathrm{H}]^{+}$213.0764; found 213.0762 . 


\section{Azidation of $\beta$-keto ester}

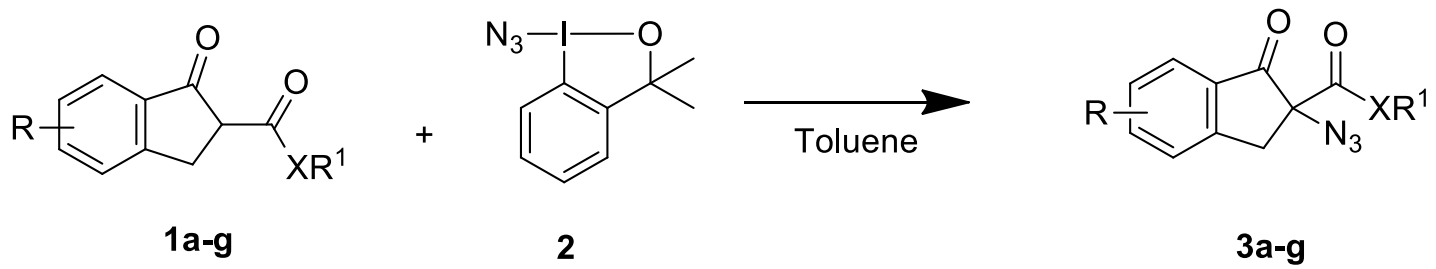

\section{General procedure for the azidation of cyclic $\beta$-keto esters.}

The cyclic $\beta$-keto ester $(0.40 \mathrm{mmol})$ was dissolved in a $2 \mathrm{~mL}$ essay tube in toluene $(1 \mathrm{M}$ in keto ester). The reaction was stirred for 1 minute before adding dimethyl azido benziodoxole 2 (0.52 mmol, 1.3 equiv) in one portion. The reaction was followed by TLC (using the solvent indicated for the $\mathrm{R}_{\mathrm{f}}$ value, visualization with UV or $p$-anisaldehyde stain) until complete consumption of the starting material (1-3 h). The crude mixture was purified by column chromatography using the solvent indicated for the $\mathbf{R}_{\mathbf{f}}$ value.

\section{Methyl 2-azido-5-methoxy-1-oxo-2,3-dihydro-1H-indene-2-carboxylate (3a)}

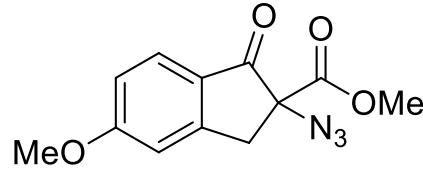

$3 a$

Starting from methyl 5-methoxy-1-oxo-2,3-dihydro- $1 H$-indene-2carboxylate (1a) $(0.089 \mathrm{~g}, 0.40 \mathrm{mmol})$, methyl 2-azido-5-methoxy-1oxo-2,3-dihydro- $1 H$-indene-2-carboxylate (3a) $(0.10 \mathrm{~g}, 0.38 \mathrm{mmol}$, $94 \%$ yield) was obtained as a yellow oil. Rf: 0.6 (DCM/Pentane 4:1). ${ }^{1} \mathbf{H}$ NMR $\left(400 \mathrm{MHz}, \mathrm{CDCl}_{3}\right) \delta 7.72(\mathrm{~d}, 1 \mathrm{H}, J=8.6 \mathrm{~Hz}, \mathrm{CHAr}), 6.95$ (dd, $1 \mathrm{H}, J=8.6,2.0 \mathrm{~Hz}, \mathrm{CHAr}), 6.87$ (s, $1 \mathrm{H}, \mathrm{CHAr}), 3.89$ (s, $3 \mathrm{H}$,

$\mathrm{OMe}$ ), 3.77 (s, $3 \mathrm{H}, \mathrm{OMe}$ ), 3.60 (d, $\left.1 \mathrm{H}, J=17.4 \mathrm{~Hz}, \mathrm{CH}_{2}\right), 2.95\left(\mathrm{~d}, 1 \mathrm{H}, J=17.4 \mathrm{~Hz}, \mathrm{CH}_{2}\right) .{ }^{{ }^{13} \mathrm{C}}$ NMR $\left(101 \mathrm{MHz}, \mathrm{CDCl}_{3}\right) \delta$ 195.2, 169.2, 166.8, 155.2, 127.5, 126.0, 116.7, 109.6, 70.6, 55.9, 53.5, 38.5. IR $v_{\max } 2956(\mathrm{w}), 2844(\mathrm{w}), 2108(\mathrm{~m}), 2108(\mathrm{~m}), 1750(\mathrm{~m}), 1710(\mathrm{~m}), 1598(\mathrm{~s}), 1493$ (w), $1447(\mathrm{w}), 1438(\mathrm{w}), 1342(\mathrm{w}), 1311(\mathrm{~m}), 1265(\mathrm{~s}), 1239(\mathrm{~m}), 1198(\mathrm{w}), 1181(\mathrm{w}), 1151(\mathrm{w})$, 1099 (w), 1089 (m), 1053 (w), 1025 (w), 960 (w), 930 (w), 900 (w), 850 (w), 832 (w), 788 (w), 751 (w). HRMS (ESI) calcd for $\mathrm{C}_{12} \mathrm{H}_{11} \mathrm{~N}_{3} \mathrm{O}_{4}{ }^{+}[\mathrm{M}]^{+}$262.0828; found 262.0830.

\section{Methyl 2-azido-1-oxo-2,3-dihydro-1H-indene-2-carboxylate (3b)}<smiles>COC(=O)C1(N)Cc2ccccc2C1=O</smiles>

3b

Starting from methyl 1-oxo-2,3-dihydro- $1 H$-indene-2-carboxylate (1b) (0.077 g, $0.40 \mathrm{mmol})$, methyl 2-azido-1-oxo-2,3-dihydro- $1 H$-indene-2carboxylate (3b) $(0.074 \mathrm{~g}, 0.32 \mathrm{mmol}, 79 \%$ yield) was obtained as a yellow oil. $\mathrm{R}_{\mathrm{f}}: 0.47$ (hexanes:EtOAc 4:1). ${ }^{1} \mathbf{H}$ NMR (400 MHz, $\left.\mathrm{CDCl}_{3}\right) \delta 7.81$ (dd, $1 \mathrm{H}, J=7.7,0.2 \mathrm{~Hz}, \mathrm{CHAr}), 7.68$ (m, $1 \mathrm{H}, \mathrm{CHAr}), 7.45$ (m, $2 \mathrm{H}, \mathrm{CHAr}$ ), 3.79 (s, $3 \mathrm{H}, \mathrm{OMe}$ ), 3.67 (d, $\left.1 \mathrm{H}, J=17.4 \mathrm{~Hz}, \mathrm{CH}_{2}\right), 3.03(\mathrm{~d}, 1 \mathrm{H}, J=17.4$ $\left.\mathrm{Hz}, \mathrm{CH}_{2}\right) .{ }^{13} \mathrm{C}$ NMR $\left(101 \mathrm{MHz}, \mathrm{CDCl}_{3}\right) \delta 197.4,169.0,152.1,136.6,133.0,128.5,126.5,125.6$, 70.2, 53.6, 38.5. IR $v_{\max } 2110$ (s), 1749 (s), 1718 (s), 1607 (w), 1464 (w), 1432 (w), 1334 (w), 1269 (s), 1233 (s), 1181 (m), 1088 (w), 1051 (s), 959 (m), 910 (m), 863 (w), 811 (w). HRMS (APPI) calcd for $\quad \mathrm{C}_{11} \mathrm{H}_{10} \mathrm{NO}_{3}{ }^{+} \quad\left[\mathrm{M}-\mathrm{N}_{2}+\mathrm{H}\right]^{+}$204.0655; found 204.0666. 


\section{Methyl 2-azido-5-fluoro-1-oxo-2,3-dihydro-1H-indene-2-carboxylate (3c)}

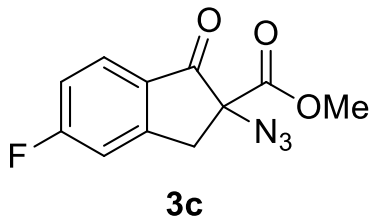

$3 \mathrm{C}$

Starting from methyl 5-fluoro-1-oxo-2,3-dihydro- $1 H$-indene-2carboxylate (1c) $(0.083 \mathrm{~g}, 0.40 \mathrm{mmol})$, methyl 2-azido-5-fluoro-1-oxo2,3-dihydro-1H-indene-2-carboxylate (3c) (0.1 g, 0.4 mmol, quant.) was obtained as a white crystalline solid. Mp $98.4-100.7^{\circ} \mathrm{C}$. $\mathbf{R f}_{\mathbf{f}}: 0.6$ (DCM/pentane 4:1). ${ }^{1} \mathbf{H}$ NMR $\left(400 \mathrm{MHz}, \mathrm{CDCl}_{3}\right) \delta 7.84(\mathrm{dd}, 1 \mathrm{H}, J=$ 8.3, $5.3 \mathrm{~Hz}, \mathrm{CHAr}), 7.16$ (m, $2 \mathrm{H}, \mathrm{CHAr}), 3.81$ (s, $3 \mathrm{H}, \mathrm{OMe}$ ), 3.66 (d, 1 $\left.\mathrm{H}, J=17.6 \mathrm{~Hz}, \mathrm{CH}_{2}\right), 3.02\left(\mathrm{~d}, 1 \mathrm{H}, J=17.6 \mathrm{~Hz}, \mathrm{CH}_{2}\right) .{ }^{13} \mathbf{C} \mathbf{N M R}\left(101 \mathrm{MHz}, \mathrm{CDCl}_{3}\right) \delta 195.5$, $168.7,168.1\left(\mathrm{~d}, J_{C-F}=260 \mathrm{~Hz}\right), 155.1\left(\mathrm{~d}, J_{C-F}=11 \mathrm{~Hz}\right), 129.4\left(\mathrm{~d}, J_{C-F}=2 \mathrm{~Hz}\right), 128.2\left(\mathrm{~d}, J_{C-F}=\right.$ $11 \mathrm{~Hz}), 117.0\left(\mathrm{~d}, J_{C-F}=24 \mathrm{~Hz}\right), 113.4\left(\mathrm{~d}, J_{C-F}=23 \mathrm{~Hz}\right), 70.4,53.7,38.4$. IR $v_{\max } 2959(\mathrm{w}), 2109$ (s), 1753 (s), 1721 (s), $1484(\mathrm{w}), 1428$ (w), 1336 (w), 1258 (s), 1199 (m), 1180 (m), 1133 (w), $1053(\mathrm{~m}), 961(\mathrm{w}), 905(\mathrm{w}), 863(\mathrm{~m}), 833$ (w), $788(\mathrm{w}), 739$ (w). HRMS (ESI) calcd for $\mathrm{C}_{11} \mathrm{FH}_{9} \mathrm{NO}_{3}{ }^{+}\left[\mathrm{M}-\mathrm{N}_{2}+\mathrm{H}\right]^{+} 222.0561$; found 222.0562 .

\section{Methyl 2-azido-6-chloro-1-oxo-2,3-dihydro-1H-indene-2-carboxylate (3d)}

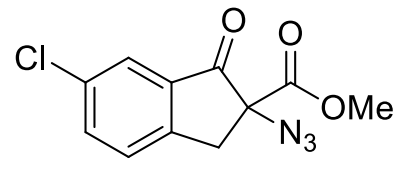

$3 \mathbf{d}$

Starting from methyl 6-chloro-1-oxo-2,3-dihydro- $1 H$-indene-2carboxylate (1d) $(0.0916 \mathrm{~g}, 0.408 \mathrm{mmol})$, methyl 2-azido-6-chloro-1oxo-2,3-dihydro- $1 \mathrm{H}$-indene-2-carboxylate (3d) $(0.10 \mathrm{~g}, 0.38 \mathrm{mmol}, 92$ $\%$ yield) was obtained as a white solid. Mp 141.6-146.3 ${ }^{\circ} \mathrm{C} . \mathrm{R}_{\mathrm{f}}: 0.66$ (DCM/pentane 4:1). ${ }^{1} \mathbf{H}$ NMR $\left(400 \mathrm{MHz}, \mathrm{CDCl}_{3}\right) \delta 7.77$ (d, $1 \mathrm{H}, J=$ $1.5 \mathrm{~Hz}, \mathrm{CHAr}), 7.63$ (dd, $1 \mathrm{H}, J=8.2,1.9 \mathrm{~Hz}, \mathrm{CHAr}), 7.42(\mathrm{~d}, 1 \mathrm{H}, J=$ $8.2 \mathrm{~Hz}, \mathrm{CHAr}), 3.80$ (s, $3 \mathrm{H}, \mathrm{OMe}$ ), $3.63\left(\mathrm{~d}, 1 \mathrm{H}, J=17.4 \mathrm{~Hz}, \mathrm{CH}_{2}\right), 3.00$ (d, $1 \mathrm{H}, J=17.5 \mathrm{~Hz}$, $\left.\mathrm{CH}_{2}\right) .{ }^{13} \mathrm{C}$ NMR $\left(101 \mathrm{MHz}, \mathrm{CDCl}_{3}\right) \delta 196.3,168.5,150.1,136.5,134.9,134.5,127.7,125.3$, 70.6, 53.7, 38.2. IR $v_{\max } 2959$ (w), 2107 (s), 1746 (s), 1720 (s), 1599 (w), 1470 (w), 1427 (m), 1327 (w), 1290 (w), 1276 (m), 1251 (s), 1237 (s), 1200 (s), 1176 (m), 1129 (w), 1108 (w), 1053 (m), 1035 (w), 955 (w), 939 (w), 908 (w), 881 (w), 832 (m), 791 (w), 741 (w), 713 (m). HRMS (ESI) calcd for $\mathrm{C}_{11} \mathrm{ClH}_{9} \mathrm{NO}_{3}{ }^{+}\left[\mathrm{M}-\mathrm{N}_{2}+\mathrm{H}\right]^{+} 238.0265$; found 238.0280.

\section{Tert-butyl 2-azido-1-oxo-2,3-dihydro-1H-indene-2-carboxylate (3e)}

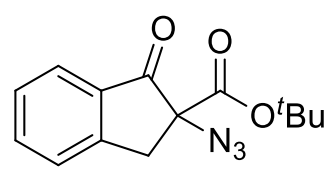

$3 e$

Starting from tert-butyl 1-oxo-2,3-dihydro-1H-indene-2-carboxylate (1e) (0.094 g, $0.40 \mathrm{mmol})$, tert-butyl 2-azido-1-oxo-2,3-dihydro- $1 H$-indene-2carboxylate (3e) $(0.11 \mathrm{~g}, 0.40 \mathrm{mmol}$, quant.) was obtained as a white solid. Rf: $0.70\left(\mathrm{DCM} /\right.$ pentane 4:1). ${ }^{1} \mathrm{H}$ NMR $\left(400 \mathrm{MHz}, \mathrm{CDCl}_{3}\right) \delta 7.79(\mathrm{~d}, 1 \mathrm{H}, J$ $=7.7 \mathrm{~Hz}, \mathrm{CHAr}), 7.65$ (td, $1 \mathrm{H}, J=7.6,1.1 \mathrm{~Hz}, \mathrm{CHAr}), 7.43$ (m, $2 \mathrm{H}$, CHAr), $3.62\left(\mathrm{~d}, 1 \mathrm{H}, J=17.2 \mathrm{~Hz}, \mathrm{CH}_{2}\right), 2.98\left(\mathrm{~d}, 1 \mathrm{H}, J=17.2 \mathrm{~Hz}, \mathrm{CH}_{2}\right)$, $1.44\left(\mathrm{~s}, 9 \mathrm{H},{ }^{t} \mathrm{Bu}\right) .{ }^{13} \mathrm{C}$ NMR $\left(101 \mathrm{MHz}, \mathrm{CDCl}_{3}\right) \delta 198.0,167.3,152.3,136.3,133.2,128.3$, 126.4, 125.4, 84.5, 70.5, 38.5, 27.9 (3C). IR $v_{\max } 2981(\mathrm{w}), 2915$ (w), 2856 (w), $2114(\mathrm{~s}), 1741$ (s), 1716 (s), 1608 (w), $1591(\mathrm{w}), 1465$ (w), $1428(\mathrm{w}), 1396(\mathrm{w}), 1371(\mathrm{w}), 1344(\mathrm{w}), 1263(\mathrm{~m})$, 1248 (s), 1236 (s), 1216 (m), $1184(\mathrm{~m}), 1152$ (s), 1106 (w), $1091(\mathrm{w}), 1049$ (m), 964 (w), $911(\mathrm{~s})$, $878(\mathrm{w}), 864(\mathrm{w}), 839(\mathrm{~m}), 809(\mathrm{w}), 792(\mathrm{w}), 733(\mathrm{~s})$. NMR data correspond to the reported values. $^{22}$ 


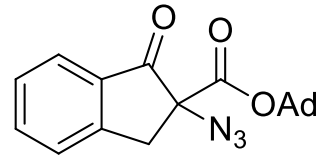

$3 \mathbf{f}$

Starting from adamantan-1-yl 1-oxo-2,3-dihydro- $1 H$-indene-2-carboxylate (1f) $(0.125 \mathrm{~g}, 0.402 \mathrm{mmol})$, adamantan-1-yl 2-azido-1-oxo-2,3-dihydro- $1 \mathrm{H}$ indene-2-carboxylate (3f) $(0.14 \mathrm{~g}, 0.39 \mathrm{mmol}, 98 \%$ yield) was obtained as a colorless oil. $\mathrm{R}_{\mathrm{f}}: 0.77$ (DCM/pentane 4:1). ${ }^{1} \mathrm{H}$ NMR $\left(400 \mathrm{MHz}, \mathrm{CDCl}_{3}\right) \delta$ 7.79 (d, $1 \mathrm{H}, J=7.7 \mathrm{~Hz}, \mathrm{CHAr}), 7.64$ (td, $1 \mathrm{H}, J=7.6,1.2 \mathrm{~Hz}, \mathrm{CHAr}), 7.42$ (m, $2 \mathrm{H}, \mathrm{CHAr}), 3.62\left(\mathrm{~d}, 1 \mathrm{H}, J=17.2 \mathrm{~Hz}, \mathrm{CH}_{2}\right), 2.97$ (d, $1 \mathrm{H}, J=17.2 \mathrm{~Hz}$, $\left.\mathrm{CH}_{2}\right), 2.13$ (s, $\left.3 \mathrm{H}, \mathrm{AdCH}\right), 2.05\left(\mathrm{~m}, 6 \mathrm{H}, \mathrm{AdCH}_{2}\right), 1.61\left(\mathrm{~m}, 6 \mathrm{H}, \mathrm{AdCH}_{2}\right) .{ }^{13} \mathrm{C} \mathrm{NMR}(101 \mathrm{MHz}$, $\left.\mathrm{CDCl}_{3}\right) \delta 198.1,166.9,152.3,136.2,133.2,128.3,126.4,125.4,84.5,70.5,41.1$ (3C), 38.6, 36.0 (3C), 30.9 (3C). IR $v_{\max } 2915$ (m), 2857 (w), 2115 (m), 1741 (s), 1718 (s), 1610 (w), 1463 (w), 1428 (w), 1353 (w), 1321 (w), 1267 (m), 1234 (s), 1188 (m), $1157(w), 1130(w), 1105(w), 1049$ (m), 964 (w), $910(\mathrm{~s}), 877$ (w), 865 (w), 837 (w), 808 (w), 733 (s). HRMS (APPI) calcd for $\mathrm{C}_{20} \mathrm{H}_{21} \mathrm{NO}_{3}\left[\mathrm{M}-\mathrm{N}_{2}\right]^{+}$323.1521; found 323.1514.

\section{2-Azido-N-(4-fluorophenyl)-1-oxo-2,3-dihydro-1H-indene-2-carboxamide (3g)}

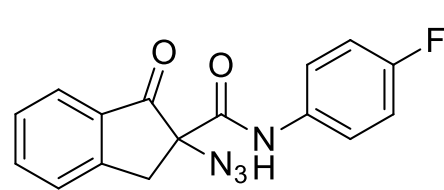

$3 g$

Starting from N-(4-fluorophenyl)-1-oxo-2,3-dihydro- $1 H$-indene-2carboxamide $(\mathbf{1 g})(0.11 \mathrm{~g}, 0.40 \mathrm{mmol}), 2$-azido-N-(4-fluorophenyl)1-oxo-2,3-dihydro-1H-indene-2-carboxamide (3g) (0.083 g, 0.27 mmol, $67 \%$ yield) was obtained as a white solid. Mp 133.3135. $1^{\circ} \mathrm{C} . \mathbf{R}_{\mathbf{f}}: 0.43(\mathrm{DCM} /$ pentane $4: 1) .{ }^{1} \mathbf{H}$ NMR $\left(400 \mathrm{MHz}, \mathrm{CDCl}_{3}\right)$ $\delta 8.53(\mathrm{~s}, 1 \mathrm{H}, \mathrm{NH}), 7.83(\mathrm{~d}, 1 \mathrm{H}, J=7.7 \mathrm{~Hz}, \mathrm{CHAr}), 7.71(\mathrm{~m}, 1 \mathrm{H}$, CHAr), 7.52 (m, 3 H, CHAr), 7.46 (t, $1 \mathrm{H}, J=7.4 \mathrm{~Hz}, \mathrm{CHAr}), 7.02$ (m, $2 \mathrm{H}, \mathrm{CHAr}), 4.11$ (d, $1 \mathrm{H}$, $\left.J=17.4 \mathrm{~Hz}, \mathrm{CH}_{2}\right), 3.32\left(\mathrm{~d}, 1 \mathrm{H}, J=17.4 \mathrm{~Hz}, \mathrm{CH}_{2}\right) .{ }^{13} \mathbf{C}$ NMR $\left(101 \mathrm{MHz}, \mathrm{CDCl}_{3}\right) \delta$ 198.0, 164.1, $159.8\left(\mathrm{~d}, J_{C-F}=245 \mathrm{~Hz}\right), 152.4,137.0,133.3,132.9(\mathrm{~d}, J=2 \mathrm{~Hz}), 128.6,126.5,125.6,122.0(\mathrm{~d}$, $J=8 \mathrm{~Hz}, 2 \mathrm{C}), 115.8(\mathrm{~d}, J=23 \mathrm{~Hz}, 2 \mathrm{C}), 73.2,37.5$. IR $v_{\max } 3384(\mathrm{w}), 3333(\mathrm{w}), 3075(\mathrm{w}), 2982$ (w), 2926 (w), 2113 (s), 1717 (s), 1679 (m), 1608 (m), 1591 (w), 1530 (s), 1509 (s), 1468 (m), 1428 (w), 1409 (m), 1372 (w), 1325 (w), $1304(\mathrm{~m}), 1277$ (m), 1248 (m), 1214 (s), $1188(\mathrm{w}), 1156$ (m), 1102 (w), $1091(\mathrm{w}), 1046(\mathrm{~m}), 1017(\mathrm{w}), 991(\mathrm{w}), 962(\mathrm{w}), 911(\mathrm{~s}), 877(\mathrm{w}), 862(\mathrm{w}), 835$ (s), $813(\mathrm{~m}), 793$ (w), 733 (s). HRMS (ESI) calcd for $\mathrm{C}_{16} \mathrm{FH}_{12} \mathrm{~N}_{4} \mathrm{O}_{2}{ }^{+}[\mathrm{M}+\mathrm{H}]^{+}$311.0939; found 311.0937 .

Tert-butyl 2-azido-1-oxo-1,2,3,4-tetrahydronaphthalene-2-carboxylate (18) and tert-butyl 1hydroxy-2-naphthoate (19)

Starting from ketoester 15 (98 mg, $0.40 \mathrm{mmol})$, azide $18(11 \mathrm{mg}, 0.038 \mathrm{mmol}, 10 \%$ yield $)$ was obtained as a colorless oil. The major product was naphthol 19 (56 mg, $0.23 \mathrm{mmol}, 57 \%$ ), isolated as a colorless oil.

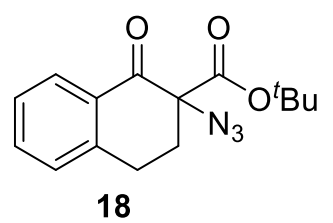

$\mathbf{R}_{\mathbf{f}} 0.33$ (100:1 Pentane/ethylacetate). ${ }^{1} \mathbf{H}$ NMR (400 MHz, $\left.\mathrm{CDCl}_{3}\right) \delta 8.07$ $(\mathrm{dd}, J=7.9,1.4 \mathrm{~Hz}, 1 \mathrm{H}, \mathrm{Ar} \mathrm{H}), 7.52(\mathrm{td}, J=7.5,1.5 \mathrm{~Hz}, 1 \mathrm{H}, \mathrm{Ar} \mathrm{H}), 7.38-$ 7.32 (m, $1 \mathrm{H}$, Ar H), $7.27-7.23$ (m, $1 \mathrm{H}$, Ar H), 3.10 (ddd, $J=17.1,8.8$, $\left.4.8 \mathrm{~Hz}, 1 \mathrm{H}, \mathrm{CH}_{2}\right), 2.99-2.89\left(\mathrm{~m}, 1 \mathrm{H}, \mathrm{CH}_{2}\right), 2.57$ (ddd, $J=13.6,8.8,4.7$ $\mathrm{Hz}, 1 \mathrm{H}, \mathrm{CH}_{2}$ ), 2.17 (ddd, $\left.J=13.8,6.6,4.8 \mathrm{~Hz}, 1 \mathrm{H}, \mathrm{CH}_{2}\right), 1.47$ (s, $9 \mathrm{H}$, $\left.{ }^{t} \mathrm{Bu}\right) .{ }^{13} \mathrm{C}$ NMR $\left(101 \mathrm{MHz}, \mathrm{CDCl}_{3}\right) \delta 190.0,167.4,143.3,134.4,130.3$, 128.7, 128.4, 127.2, 84.2, 71.0, 31.4, 27.9, 25.0. IR v $2980(\mathrm{w}), 2934$ (w), 2109 (s), $1740(\mathrm{~s})$, 1683 (m), 1602 (w), 1479 (w), 1456 (w), 1371 (w), 1277 (m), 1237 (s), 1153 (s), 1069 (w), $934(\mathrm{w}), 909(\mathrm{~m}), 841(\mathrm{w}), 820(\mathrm{w})$. NMR data correspond to the reported values. ${ }^{23}$ 


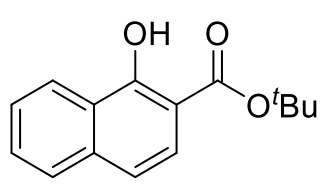

19

$\mathbf{R}_{\mathbf{f}} 0.97$ (100:1 Pentane/ethylacetate). ${ }^{1} \mathbf{H}$ NMR (400 MHz, $\left.\mathrm{CDCl}_{3}\right) \delta 12.27$ (s, $1 \mathrm{H}, \mathrm{OH}), 8.40$ (ddt, $J=8.4,1.5,0.8 \mathrm{~Hz}, 1 \mathrm{H}$, Ar H), $7.77-7.74$ (m, 1 $\mathrm{H}, \operatorname{Ar} \mathrm{H}), 7.72(\mathrm{~d}, J=8.8 \mathrm{~Hz}, 1 \mathrm{H}, \mathrm{Ar} \mathrm{H}), 7.59$ (ddd, $J=8.2,6.9,1.4 \mathrm{~Hz}, 1$ $\mathrm{H}, \mathrm{Ar} \mathrm{H}), 7.51$ (ddd, $J=8.2,6.9,1.3 \mathrm{~Hz}, 1 \mathrm{H}, \mathrm{Ar} \mathrm{H}), 7.25$ (d, $J=8.6 \mathrm{~Hz}, 1$ $\mathrm{H}, \mathrm{Ar} \mathrm{H}), 1.65\left(\mathrm{~s}, 9 \mathrm{H},{ }^{t} \mathrm{Bu}\right)$. NMR data correspond to the reported values. ${ }^{24}$

\section{Methyl 1-azido-2-oxocyclopentane-1-carboxylate (20)}

Starting from ketoester 17 (73 mg, $0.40 \mathrm{mmol})$, azide 20 (39.5 mg, $0.216 \mathrm{mmol}, 54 \%$ yield) was obtained as a colorless oil.

$\mathbf{R}_{\mathbf{f}} 0.5$ (6:4 Pentane/DCM). ${ }^{1} \mathbf{H}$ NMR $\left(400 \mathrm{MHz}, \mathrm{CDCl}_{3}\right) \delta 3.83(\mathrm{~s}, 3 \mathrm{H}, \mathrm{OMe}), 2.52$<smiles>COC(=O)C1(N)CCCC1=O</smiles>

20 - $2.35\left(\mathrm{~m}, 3 \mathrm{H}, \mathrm{CH}_{2}\right), 2.16-1.92\left(\mathrm{~m}, 3 \mathrm{H}, \mathrm{CH}_{2}\right) .{ }^{13} \mathbf{C ~ N M R}\left(101 \mathrm{MHz}, \mathrm{CDCl}_{3}\right) \delta$ 208.9, 169.0, 70.9, 53.4, 36.5, 34.2, 19.2. IR $v 2976(\mathrm{w}), 2959(\mathrm{w}), 2897(\mathrm{w}), 2159$ (w), $2111(\mathrm{~s}), 1753$ (s), 1739 (s), $1438(\mathrm{w}), 1404(\mathrm{w}), 1317(\mathrm{w}), 1270(\mathrm{~m}), 1230(\mathrm{~s})$, 1194 (w), 1162 (m), 1143 (m), 1127 (m), 1041 (w), 1004 (m), 961 (w), 917 (w), 844 values. $^{25}$ (w), $819(\mathrm{w}), 797(\mathrm{w}), 765(\mathrm{w}), 738(\mathrm{w}) .{ }^{1} \mathrm{H}$ NMR data correspond to the reported 
5. Zinc-catalyzed azidation of linear $\beta$-keto esters.<smiles>[R]C(=O)C(C)C(=O)OC</smiles>

$1 \mathrm{~h}-\mathbf{k}$<smiles>CC1(C)OI(N)c2ccccc21</smiles>

2

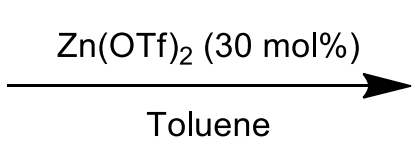<smiles>[R]C(=O)C(C)(C)C(=O)OC</smiles>

3h-k

\section{General procedure}

$\mathrm{Zn}(\mathrm{OTf})_{2}(0.021 \mathrm{~g}, 0.058 \mathrm{mmol}, 0.030$ equiv $)$ was suspended in a $2 \mathrm{~mL}$ essay tube in toluene $(0.5$ M). The linear $\beta$-keto ester $(\mathbf{1 h}-\mathbf{k})(0.40 \mathbf{~ m m o l})$ was then added. The reaction mixture was stirred for one minute before adding in one portion the dimethyl azido benziodoxole $2(0.15 \mathrm{~g}, 0.50$ mmol, 1.3 equiv). The reaction was stirred at room and followed by TLC (using the solvent indicated for the $\mathrm{R}_{\mathrm{f}}$ value, visualization with $\mathrm{UV}$ or $p$-anisaldehyde stain) until complete consumption of the starting material $(12-24 \mathrm{~h})$. The crude mixture was purified by column chromatography, using the solvent indicated for the $\mathbf{R}_{\mathbf{f}}$ value.

Methyl 2-azido-2-methyl-3-oxo-3-phenylpropanoate (3h)<smiles>COC(=O)C(C)(N)C(=O)c1ccccc1</smiles>

3h

Starting from methyl methyl 2-methyl-3-oxo-3-phenylpropanoate (0.078 g, $0.406 \mathrm{mmol})$ (1h), methyl 2-azido-2-methyl-3-oxo-3-phenylpropanoate (0.069 g, $0.296 \mathrm{mmol}, 73 \%$ yield, > 95\% pure by NMR) (3h) was obtained as a yellow oil. Rf: 0.3 (Pentane/ethyl acetate 10:1). ${ }^{1} \mathbf{H}$ NMR (400 MHz, $\left.\mathrm{CDCl}_{3}\right) \delta 7.97$ (m, $\left.2 \mathrm{H}, \mathrm{C} \underline{H A r}\right), 7.57$ (m, $\left.1 \mathrm{H}, \mathrm{C} \underline{H A r}\right), 7.45$ (m, $2 \mathrm{H}, \mathrm{C} \underline{H A r}), 3.75$ (s, $3 \mathrm{H}, \mathrm{OMe}), 1.82$ (s, $\left.3 \mathrm{H}, \mathrm{C}_{3}\right)$ ). ${ }^{13} \mathrm{C} \mathbf{N M R}(101 \mathrm{MHz}$, $\left.\mathrm{CDCl}_{3}\right) \delta 190.9,170.3,133.8,133.5,129.4(2 \mathrm{C}), 128.7(2 \mathrm{C}), 71.1,53.5,20.3 . \mathbf{I R} v_{\max } 3069(\mathrm{w})$, 3068 (w), 3007 (w), 2957 (w), 2111 (s), 1749 (s), 1696 (s), 1598 (w), 1582 (w), 1515 (w), 1450 (m), $1411(\mathrm{w}), 1379$ (w), 1355 (w), 1235 (s), 1189 (w), 1120 (s), 1062 (w), 1002 (w), 967 (m), $912(\mathrm{~m}), 864(\mathrm{w}), 799(\mathrm{w}), 786(\mathrm{w}), 733(\mathrm{~s})$. NMR data correspond to the reported values. ${ }^{26}$

\section{Methyl 2-azido-2-methyl-3-oxo-3-(4-(trifluoromethyl)phenyl)propanoate (3i)}<smiles>COC(=O)C(C)(N)C(=O)c1ccc(C(F)(F)F)cc1</smiles>

$3.76\left(\mathrm{~s}, 3 \mathrm{H}, \mathrm{OMe}_{3}\right), 1.84\left(\mathrm{~s}, 3 \mathrm{H}, \mathrm{CH}_{3}\right) .{ }^{13} \mathrm{C} \mathbf{N M R}\left(101 \mathrm{MHz}, \mathrm{CDCl}_{3}\right) \delta 190.0,170.1,136.3$, $134.8\left(\mathrm{q}, J_{C-F}=32 \mathrm{~Hz}\right), 129.8(2 \mathrm{C}), 125.7\left(\mathrm{q}, J_{C-F}=4 \mathrm{~Hz}, 2 \mathrm{C}\right), 123.4\left(\mathrm{q}, J_{C-F}=272 \mathrm{~Hz}\right), 71.0$, 53.7, 20.0. IR $v_{\max } 3081(\mathrm{w}), 3062(\mathrm{w}), 3011(\mathrm{w}), 2960(\mathrm{w}), 2849(\mathrm{w}), 2117(\mathrm{~m}), 1752(\mathrm{~m}), 1706$ (m), $1582(\mathrm{w}), 1514(\mathrm{w}), 1453(\mathrm{w}), 1440$ (w), $1411(\mathrm{w}), 1380(\mathrm{w}), 1329(\mathrm{~s}), 1246(\mathrm{~m}), 1236(\mathrm{~m})$, 1172 (s), 1119 (s), 1068 (s), 1018 (m), 972 (m), 912 (m), 854 (m), 795 (w), 767 (w), 736 (m), 711 (w). HRMS (ESI) calcd for $\mathrm{C}_{12} \mathrm{~F}_{3} \mathrm{H}_{11} \mathrm{NO}_{3}{ }^{+}\left[\mathrm{M}-\mathrm{N}_{2}+\mathrm{H}\right]^{+}$274.0686; found 274.0685.

Methyl 2-azido-3-(4-bromophenyl)-2-methyl-3-oxopropanoate (3j) 
<smiles>COC(=O)C(C)(N)C(=O)c1ccc(Br)cc1</smiles>

3j

Starting from methyl 3-(4-bromophenyl)-2-methyl-3-oxopropanoate (1j) $(0.104 \mathrm{~g}, 0.385 \mathrm{mmol})$, methyl 2-azido-3-(4-bromophenyl)-2methyl-3-oxopropanoate (3j) (0.066 g, $0.21 \mathrm{mmol}, 55 \%$ yield, > 95\%

pure by NMR) was obtained as a yellow oil. Rf: 0.3 (pentane:ethyl acetate 6:4). ${ }^{1} \mathbf{H}$ NMR (400 MHz, $\left.\mathrm{CDCl}_{3}\right) \delta 7.86$ (m, $\left.2 \mathrm{H}, \mathrm{CHAr}\right), 7.61$ (m, $2 \mathrm{H}, \mathrm{CHAr}), 3.77$ (s, $3 \mathrm{H}, \mathrm{OMe}), 1.83\left(\mathrm{~s}, 3 \mathrm{H}, \mathrm{CH}_{3}\right) .{ }^{13} \mathrm{C}$ NMR $\left(101 \mathrm{MHz}, \mathrm{CDCl}_{3}\right) \delta 189.9,170.2,132.2,132.0(2 \mathrm{C}), 130.9(2 \mathrm{C}), 129.2,71.0,53.6,20.1 . \mathbf{I R} v_{\max }$ 3358 (w), 3343 (w), 3332 (w), 2120 (s), 1752 (w), 1698 (s), 1600 (m), 1534 (s), 1447 (m), 1413 (w), $1377(\mathrm{w}), 1357(\mathrm{w}), 1327(\mathrm{~s}), 1250$ (s), $1174(\mathrm{~m}), 1134(\mathrm{~s}), 1069$ (m), $1017(\mathrm{w}), 970(\mathrm{w})$, $911(\mathrm{w}), 857$ (w), $758(\mathrm{~m}), 743(\mathrm{~m})$. HRMS (APPI) calcd for $\mathrm{C}_{11}{ }^{79} \mathrm{BrH}_{11} \mathrm{NO}_{3}{ }^{+}\left[\mathrm{M}-\mathrm{N}_{2}+\mathrm{H}\right]^{+}$ 283.9917; found 283.9932 .

\section{Methyl 2-azido-2-methyl-3-oxo-3-(thiophen-2-yl)propanoate (3k)}<smiles>COC(=O)C(C)(N)C(=O)c1cccs1</smiles>

3k

Starting from methyl 2-methyl-3-oxo-3-(thiophen-2-yl)propanoate (1k) $(0.079 \mathrm{~g}, 0.40 \mathrm{mmol})$, methyl 2-azido-2-methyl-3-oxo-3-(thiophen-2yl)propanoate $(3 \mathbf{k})(0.062 \mathrm{~g}, 0.26 \mathrm{mmol}, 64 \%$ yield $)$ was obtained as a yellow oil. Rf: 0.3 (pentane:ethyl acetate 6:4). ${ }^{1} \mathbf{H} \mathbf{~ N M R}\left(400 \mathrm{MHz}, \mathrm{CDCl}_{3}\right)$ $\delta 7.88(\mathrm{dd}, 1 \mathrm{H}, J=3.9,1.0 \mathrm{~Hz}, \mathrm{CHAr}), 7.71(\mathrm{dd}, 1 \mathrm{H}, J=5.0,1.0 \mathrm{~Hz}$, CHAr), 7.13 (dd, $1 \mathrm{H}, J=4.9,4.0 \mathrm{~Hz}, \mathrm{CHAr}$ ), 3.78 (s, $3 \mathrm{H}, O M e$ ), 1.80 (s, $\left.3 \mathrm{H}, \mathrm{CH}_{3}\right) .{ }^{13} \mathrm{C}$ NMR $\left(101 \mathrm{MHz}, \mathrm{CDCl}_{3}\right) \delta 184.5,169.7,139.4,135.5,134.4,128.5,71.9,53.6$, 20.5. IR $v_{\max } 3104(\mathrm{w}), 3006(\mathrm{w}), 2957$ (w), 2110 (s), 1748 (s), 1673 (s), 1515 (w), 1452 (w), 1436 (w), 1379 (w), 1265 (s), 1238 (s), 1118 (s), 1084 (w), 1061 (m), 975 (w), 925 (w), 863 (w), $833(\mathrm{w}), 781(\mathrm{w}), 767$ (m), 729 (s). HRMS (ESI) calcd for $\mathrm{C}_{9} \mathrm{H}_{10} \mathrm{~N}_{3} \mathrm{O}_{3} \mathrm{~S}^{+}[\mathrm{M}+\mathrm{H}]^{+} 240.0437$; found 240.0444 . 


\section{Complete optimization table for silyl enol ether azidation.}<smiles>[MgH]OC1=CCc2ccccc21</smiles>

4a-c<smiles>CC1(C)OI(N)c2ccccc21</smiles>

2

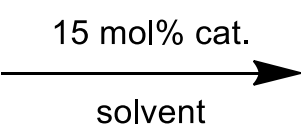

The Lewis Acid (15 mol \%) was suspended in a test tube of $2 \mathrm{~mL}$ in toluene (0.05 M). Silyl enol ether 4a-c $(0.1 \mathrm{mmol})$ was added and the reaction was stirred for 1 minute. Then dimethyl azido benziodoxole $2(0.13 \mathrm{mmol}, 1.3$ equiv) was added in one portion. The reaction was followed by TLC (using the solvent indicated for the $\mathrm{R}_{\mathrm{f}}$ value, visualization with UV or $p$-anisaldehyde stain) until disappearance of the starting material.

Without further purification a sample of $0.2 \mathrm{~mL}$ was dried under high vacuum. A known amount of trimethoxybenzene $(1.7 \mathrm{mg}, 0.010 \mathrm{mmol}$, external standard) was added to the crude mixture. The yield was determined by ${ }^{1} \mathrm{HNMR}$ analysis comparison with 1,3,5trimethoxybenzene peak at $6.2 \mathrm{ppm}$ and the product peak at $4.32 \mathrm{ppm}$.

\section{Calibration curve with 1,3,5-trimethoxybenzene.}

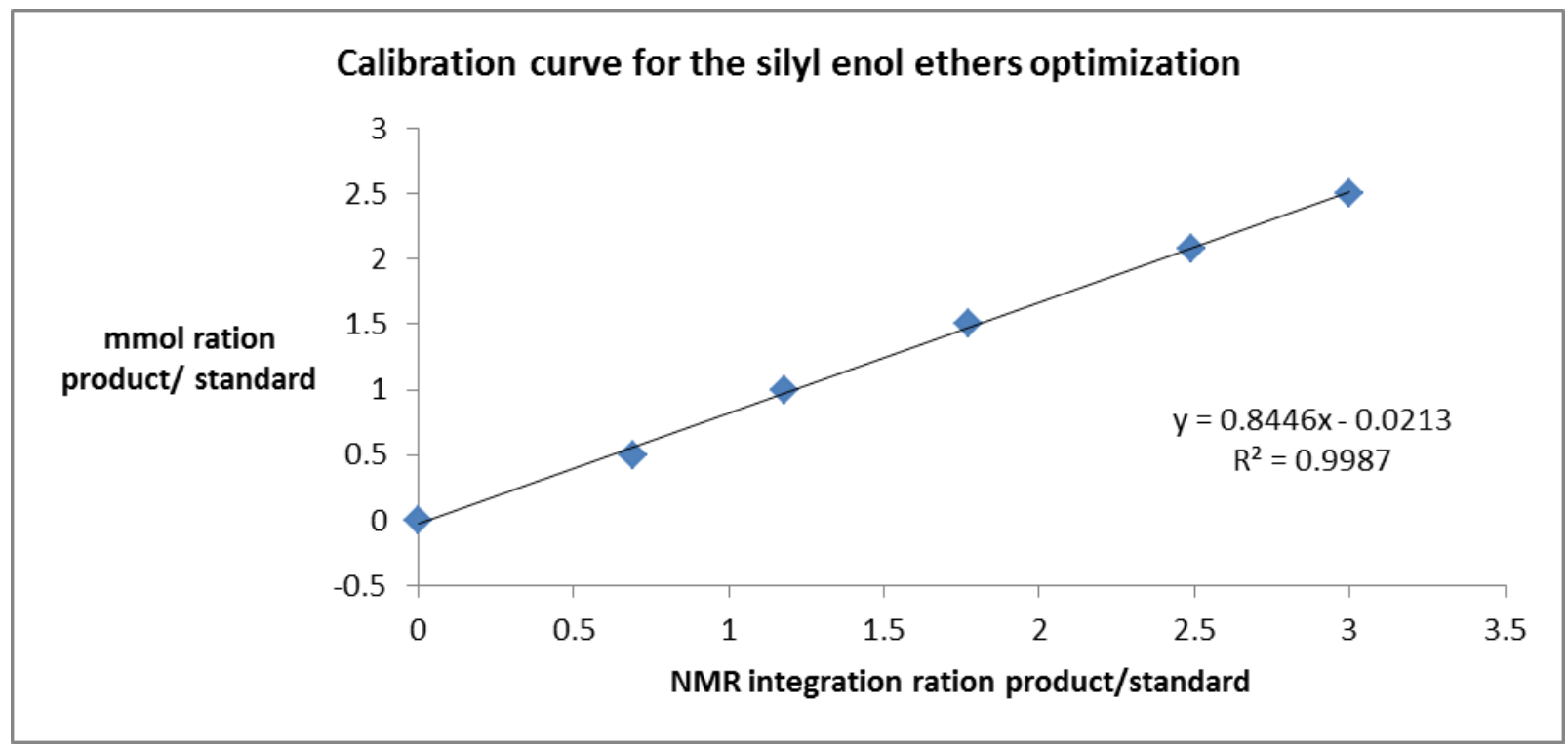

$\underline{\text { Solvent and temperature optimization. }}$

\begin{tabular}{|c|c|c|}
\hline $\begin{array}{c}\text { Solvent } \\
\text { With Zn(OTf })_{2}\end{array}$ & Temperature & $\begin{array}{c}\mathbf{1}^{\mathbf{H N M R}} \\
\text { yields \% }\end{array}$ \\
\hline Toluene & $\mathbf{r t}$ & $\mathbf{6 7}$ \\
\hline $\mathrm{Et}_{2} \mathrm{O}$ & $\mathrm{rt}$ & 52 \\
\hline $\mathrm{CH}_{3} \mathrm{CN}$ & $\mathrm{rt}$ & 53 \\
\hline $\mathrm{iPrOH}$ & $\mathrm{rt}$ & 0 \\
\hline $\mathrm{DMF}$ & $\mathrm{rt}$ & 10 \\
\hline $\mathrm{DMSO}$ & $\mathrm{rt}$ & 30 \\
\hline $\mathrm{DCM}$ & $\mathrm{rt}$ & 30 \\
\hline
\end{tabular}




\begin{tabular}{|c|c|c|}
\hline THF & rt & 26 \\
\hline $0^{\circ} \mathrm{C}$ & $0^{\circ} \mathrm{C}$ & 22 \\
\hline
\end{tabular}

Lewis acid optimization.

\begin{tabular}{|c|c|}
\hline $\begin{array}{c}\text { Lewis Acid } \\
\text { In toluene at r.t. }\end{array}$ & $\begin{array}{c}\text { 1 HNMR } \\
\text { yields \% }\end{array}$ \\
\hline $\mathbf{Z n}(\text { OTf })_{2}$ & $\mathbf{6 7}$ \\
\hline $\mathrm{Sc}(\mathrm{OTf})_{3}$ & 23 \\
\hline $\mathrm{Cu}(\mathrm{OTf})_{2}$ & 36 \\
\hline $\mathrm{In}(\mathrm{OTf})_{3}$ & 58 \\
\hline $\mathrm{Sn}(\mathrm{OTf})_{2}$ & 68 \\
\hline $\mathrm{Yb}(\mathrm{OTf})_{3}$ & 19 \\
\hline $\mathrm{AgOTf}$ & 25 \\
\hline $\mathrm{Hf}(\mathrm{OTf})_{4}$ & 71 \\
\hline $\mathrm{AlCl}_{3}$ & 0 \\
\hline$(\mathrm{CuOTf})_{2}$ Tol & 38 \\
\hline $\mathrm{CuCl}_{2}$ & n.d \\
\hline $\mathrm{SnCl}_{4}$ & 30 \\
\hline
\end{tabular}

$\underline{\text { Zinc salts optimization. }}$

\begin{tabular}{|c|c|}
\hline Zinc salts & $\begin{array}{c}\text { 1 HNMR } \\
\text { yields \% }\end{array}$ \\
\hline $\mathrm{Zn}(\mathbf{O T f})_{2}$ & $\mathbf{6 7 \%}$ \\
\hline $\mathrm{Zn}\left(\mathrm{HNTf}_{2}\right)_{2}$ & $41 \%$ \\
\hline $\mathrm{Zn}\left(\mathrm{OAc}_{2}\right.$ & $22 \%$ \\
\hline $\mathrm{Zn}\left(\mathrm{CF}_{3} \mathrm{CO}_{2}\right)_{2}$ & $26 \%$ \\
\hline $\mathrm{Zn}\left(\mathrm{BF}_{4}\right)_{2}$ & $47 \%$ \\
\hline $\mathrm{ZnBr}_{2}$ & $28 \%$ \\
\hline $\mathrm{ZnCl}_{2}$ & $18 \%$ \\
\hline
\end{tabular}




\section{Zinc-catalyzed azidation of silyl enol ethers.}

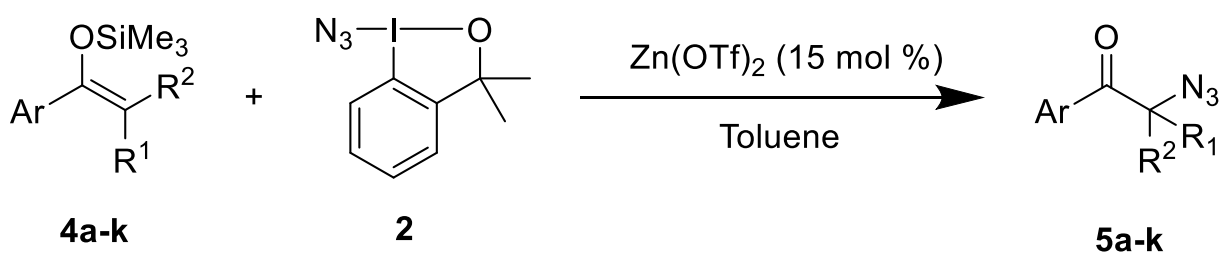

\section{General procedure}

$\mathrm{Zn}(\mathrm{OTf})_{2}(0.021 \mathrm{~g}, 0.058 \mathrm{mmol}, 0.030$ equiv) was suspended in a $2 \mathrm{~mL}$ essay tube in toluene $(0.5$ $\mathrm{M}$ in silyl enol ether). The silyl enol ether (4a-k) $(0.40 \mathrm{mmol})$ was then added. The reaction mixture was stirred for one minute before adding in one portion the dimethyl azido benziodoxole $2(0.15 \mathrm{~g}, 0.50 \mathrm{mmol}, 1.3 \mathrm{equiv})$. The reaction mixture was stirred at room temperature and followed by TLC (using the solvent indicated for the $\mathrm{R}_{\mathrm{f}}$ value, visualization with UV or $p$ anisaldehyde stain) until complete consumption of the starting material (12-24 h). The crude was purified by column chromatography, using the solvent indicated for the $\mathbf{R}_{\mathbf{f}}$ value.

\section{2-Azido-2,3-dihydro-1H-inden-1-one (5a)}

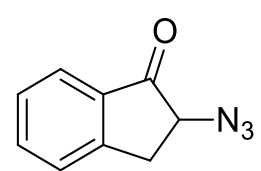

$5 a$

Starting from ((1H-inden-3-yl)oxy)trimethylsilane (4a) $(0.0794 \mathrm{~g}, \quad 0.389$ mmol), 2-azido-2,3-dihydro- $1 H$-inden-1-one (5a) $(0.0388$ g, $0.224 \mathrm{mmol}, 58 \%$ yield) was obtained as a yellow oil. Rf: 0.3 (Hexane/DCM 1:1). ${ }^{\mathbf{1}} \mathbf{H}$ NMR (400 $\left.\mathrm{MHz}^{\mathrm{CDCl}}{ }_{3}\right) \delta 7.80(\mathrm{~d}, 1 \mathrm{H}, J=7.8 \mathrm{~Hz}, \mathrm{CHAr}), 7.66(\mathrm{td}, 1 \mathrm{H}, J=7.6,1.2 \mathrm{~Hz}$, CHAr)), 7.44 (m, 2 H, CHAr)), 4.33 (dd, $1 \mathrm{H}, J=8.2,4.7 \mathrm{~Hz}, \mathrm{CH}), 3.51$ (dd, 1 $\left.\mathrm{H}, J=17.2,8.3 \mathrm{~Hz}, \mathrm{CH}_{2}\right), 2.94\left(\mathrm{dd}, 1 \mathrm{H}, J=17.1,4.6 \mathrm{~Hz}, \mathrm{CH}_{3}\right) .{ }^{13} \mathbf{C}$ NMR $\left(101 \mathrm{MHz}, \mathrm{CDCl}_{3}\right) \delta 201.8,151.2,136.1,134.2,128.3,126.6,124.7,62.0,33.0$. IR $v_{\max } 2349$ (w), 2103 (s), 1721 (s), 1610 (m), 1609 (m), 1469 (w), 1432 (w), 1304 (w), 1303 (w), 1270 (m), 1226 (m), 1225 (m), 1170 (w), 1158 (w), 1089 (w), 1088 (w), 1046 (m), 1037 (m), 1001 (m), $1000(\mathrm{~m}), 956(\mathrm{w}), 896(\mathrm{w}), 860(\mathrm{w})$. NMR data correspond to the reported values. ${ }^{27}$

\section{2-Azido-3,4-dihydronaphthalen-1(2H)-one (5d)}<smiles>NC1CCc2ccccc2C1=O</smiles>

$5 d$

Starting from ((3,4-dihydronaphthalen-1-yl)oxy)trimethylsilane (4d) (0.0883 g, $0.404 \mathrm{mmol}), 2$-azido-3,4-dihydronaphthalen-1(2H)-one (5d) $(0.044 \mathrm{~g}, 0.24$ mmol, 58\% yield) was obtained as a yellow oil. Rf: 0.3 (DCM/pentane 7:3). ${ }^{1} \mathbf{H}$ NMR $\left(400 \mathrm{MHz}, \mathrm{CDCl}_{3}\right) \delta 8.06(\mathrm{dd}, 1 \mathrm{H}, J=7.8,1.2 \mathrm{~Hz}, \mathrm{CHAr}), 7.52$ (td, 1 $\mathrm{H}, J=7.5,1.4 \mathrm{~Hz}, \mathrm{CHAr}), 7.34$ (m, $1 \mathrm{H}, \mathrm{CHAr}), 7.25$ (m, $1 \mathrm{H}, \mathrm{CHAr}$ ), 4.23 $(\mathrm{m}, 1 \mathrm{H}, \mathrm{CH}), 3.07\left(\mathrm{dd}, 2 \mathrm{H}, J=7.7,4.3 \mathrm{~Hz}, \mathrm{CH}_{2}\right), 2.37\left(\mathrm{~m}, 1 \mathrm{H}, \mathrm{CH}_{2}\right), 2.13\left(\mathrm{~m}, 1 \mathrm{H}, \mathrm{CH}_{2}\right) .{ }^{13} \mathrm{C}$ NMR $\left(101 \mathrm{MHz}, \mathrm{CDCl}_{3}\right) \delta 193.9,143.4,134.3,131.1,128.8,128.0,127.2,64.4,29.4,27.6$. IR $v_{\max } 2943(\mathrm{w}), 2873(\mathrm{w}), 2842(\mathrm{w}), 2101(\mathrm{~s}), 1730(\mathrm{w}), 1692(\mathrm{~s}), 1602(\mathrm{~m}), 1481(\mathrm{w}), 1456(\mathrm{w})$, 1435 (w), 1357 (w), 1343 (w), $1314(\mathrm{w}), 1267$ (m), 1227 (s), $1160(\mathrm{w}), 1114(\mathrm{w}), 1089(\mathrm{w}), 1026$ (w), $972(w), 916(\mathrm{~m}), 890(\mathrm{w}), 871(\mathrm{w}), 821(\mathrm{w}), 779(\mathrm{w}), 744(\mathrm{~s}), 734(\mathrm{~s})$. NMR data correspond to the reported values. ${ }^{27}$

\section{2-Azido-2-methyl-2,3-dihydro-1H-inden-1-one (5e)}

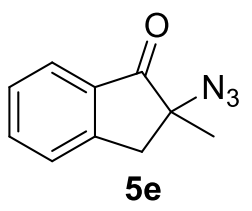

Starting from trimethyl((2-methyl-1H-inden-3-yl)oxy)silane (4e) $(0.0873 \mathrm{~g}$, $0.400 \mathrm{mmol}), 2$-azido-2-methyl-2,3-dihydro-1H-inden-1-one (5e) $(0.046 \mathrm{~g}$, $0.25 \mathrm{mmol}, 62 \%$ yield) was obtained as a yellow oil. Rf: 0.3 (DCM/pentane 
3:2). ${ }^{1} \mathbf{H}$ NMR (400 MHz, $\left.\mathrm{CDCl}_{3}\right) \delta 7.80(\mathrm{~d}, 1 \mathrm{H}, J=8.0 \mathrm{~Hz}, \mathrm{CHAr}), 7.64$ (td, $1 \mathrm{H}, J=7.5,1.1$ $\mathrm{Hz}, \mathrm{CHAr}), 7.41$ (m, $2 \mathrm{H}, \mathrm{CHAr}), 3.13$ (s, $\left.2 \mathrm{H}, \mathrm{CH}_{2}\right), 1.60$ (s, $\left.3 \mathrm{H}, \mathrm{CH}_{3}\right) .{ }^{13} \mathbf{C}$ NMR (101 MHz, $\left.\mathrm{CDCl}_{3}\right) \delta 203.2,150.8,136.1,133.5,128.2,126.6,125.2,65.3,40.9,21.2 . \mathbf{I R} v_{\max } 2973(\mathrm{w})$, $2929(\mathrm{w}), 2102(\mathrm{~s}), 1720(\mathrm{~s}), 1609(\mathrm{~m}), 1590(\mathrm{w}), 1467(\mathrm{w}), 1433(\mathrm{w}), 1375(\mathrm{w}), 1330(\mathrm{w}), 1304$ (w), $1287(\mathrm{~m}), 1250(\mathrm{~m}), 1217(\mathrm{~m}), 1173(\mathrm{w}), 1134(\mathrm{w}), 1111(\mathrm{w}), 1092(\mathrm{w}), 1071(\mathrm{w}), 970(\mathrm{~m})$, $913(\mathrm{w}), 875(\mathrm{w}), 796(\mathrm{w}), 739(\mathrm{~m})$. HRMS (ESI) calcd for $\mathrm{C}_{10} \mathrm{H}_{10} \mathrm{NO}^{+}\left[\mathrm{M}-\mathrm{N}_{2}+\mathrm{H}\right]^{+}$160.0757; found 160.0772 .

\section{2-Azido-2-methyl-3,4-dihydronaphthalen-1(2H)-one (5f)}

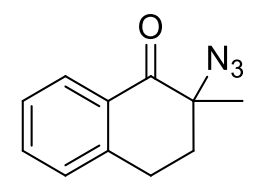

$5 f$

Starting from trimethyl((2-methyl-3,4-dihydronaphthalen-1-yl)oxy)silane (4f) (0.0952 g, $0.410 \mathrm{mmol})$, 2-azido-2-methyl-3,4-dihydronaphthalen-1(2H)-one (5f) $\left(0.045 \mathrm{~g}, 0.224 \mathrm{mmol}, 55 \%\right.$ yield) was obtained as a yellow oil. $\mathrm{R}_{\mathrm{f}}: 0.5$ (Pentane/diethyl ether 10:1). ${ }^{1} \mathbf{H}$ NMR $\left(400 \mathrm{MHz}, \mathrm{CDCl}_{3}\right) \delta 8.02(\mathrm{dd}, 1 \mathrm{H}, J=$ 7.9, 1.0 Hz, CHAr), 7.45 (td, $1 \mathrm{H}, J=7.5,1.4 \mathrm{~Hz}, \mathrm{CHAr}), 7.28$ (m, $1 \mathrm{H}, \mathrm{CHAr}$ ), $7.18(\mathrm{~d}, 1 \mathrm{H}, J=7.7 \mathrm{~Hz}, \mathrm{CHAr}), 3.08\left(\mathrm{~m}, 1 \mathrm{H}, \mathrm{CH}_{2}\right), 2.84\left(\mathrm{~m}, 1 \mathrm{H}, \mathrm{CH}_{2}\right), 2.15$ $\left(\mathrm{m}, 1 \mathrm{H}, \mathrm{CH}_{2}\right), 2.02\left(\mathrm{~m}, 1 \mathrm{H}, \mathrm{CH}_{2}\right), 1.51\left(\mathrm{~s}, 3 \mathrm{H}, \mathrm{CH}_{3}\right) .{ }^{13} \mathrm{C} \mathrm{NMR}\left(101 \mathrm{MHz}, \mathrm{CDCl}_{3}\right) \delta 194.5$, 143.3, 134.0, 130.3, 128.7, 128.6, 127.1, 64.4, 34.9, 25.6, 20.3. IR $v_{\max } 2977$ (w), 2933 (w), 2106 (s), $1729(\mathrm{w}), 1685(\mathrm{~s}), 1602(\mathrm{~m}), 1485(\mathrm{w}), 1456(\mathrm{~m}), 1434(\mathrm{w}), 1377(\mathrm{w}), 1358(\mathrm{w}), 1305(\mathrm{~m})$, 1253 (m), 1229 (s), 1158 (w), 1129 (w), 1088 (w), 1042 (w), 1018 (w), 993 (w), 967 (m), 909 (w), $896(\mathrm{w}), 875(\mathrm{w}), 800(\mathrm{w}), 778(\mathrm{w}), 705(\mathrm{w})$. HRMS (ESI) calcd for $\mathrm{C}_{11} \mathrm{H}_{12} \mathrm{NO}^{+}\left[\mathrm{M}-\mathrm{N}_{2}+\mathrm{H}\right]^{+}$ 174.0913; found 174.0911.

\section{2-Azido-1-phenylethanone (5g)}<smiles>NCC(=O)c1ccccc1</smiles>

$5 \mathrm{~g}$

Starting from trimethyl((1-phenylvinyl)oxy)silane $\mathbf{( 4 g )}(0.077 \mathrm{~g}, 0.40 \mathrm{mmol})$, 2-azido-1-phenylethanone (5g) (0.047 g, $0.29 \mathrm{mmol}, 73 \%$ yield) was obtained as a yellow oil. $\mathrm{R}_{\mathrm{f}}$ : 0.3 (Pentane/diethyl ether 9:1). ${ }^{1} \mathbf{H}$ NMR (400 MHz, $\left.\mathrm{CDCl}_{3}\right) \delta 7.91(\mathrm{~m}, 2 \mathrm{H}, \mathrm{CHAr}), 7.64(\mathrm{~m}, 1 \mathrm{H}, \mathrm{CHAr}), 7.49$ (m, $2 \mathrm{H}, \mathrm{CHAr}$ ), $4.56\left(\mathrm{~s}, 2 \mathrm{H}, \mathrm{CH}_{2}\right) .{ }^{13} \mathrm{C}$ NMR $\left(101 \mathrm{MHz}, \mathrm{CDCl}_{3}\right) \delta 193.2,134.4,134.2,129.0$ (2C), $128.0(2 \mathrm{C}), 54.9$. NMR data correspond to the reported values. ${ }^{27}$

\section{2-Azido-1-phenylpropan-1-one (5h)}<smiles>CC(N)C(=O)c1ccccc1</smiles>

$5 \mathrm{~h}$

Starting from (Z)-trimethyl((1-phenylprop-1-en-1-yl)oxy)silane (4h) (0.0662 g, $0.321 \mathrm{mmol}), 2$-azido-1-phenylpropan-1-one (5h) $(0.0405 \mathrm{~g}, 0.231 \mathrm{mmol}, 72 \%$ yield) was obtained as a yellow oil. $\mathrm{R}_{\mathrm{f}}$ : 0.3 (Pentane/ethyl acetate 10:1) ${ }^{1} \mathbf{H}$ NMR (400 MHz, $\left.\mathrm{CDCl}_{3}\right) \delta 7.94(\mathrm{~m}, 2 \mathrm{H}, \mathrm{CHAr}), 7.62$ (m, $\left.1 \mathrm{H}, \mathrm{CHAr}\right), 7.50$ (t, $2 \mathrm{H}, J=7.9 \mathrm{~Hz}, \mathrm{CHAr}), 4.71(\mathrm{q}, 1 \mathrm{H}, J=7.0 \mathrm{~Hz}, \mathrm{CHAr}), 1.57$ (d, $3 \mathrm{H}, J=$ $\left.7.0 \mathrm{~Hz}, \mathrm{CH}_{3}\right) .{ }^{13} \mathrm{C}$ NMR $\left(101 \mathrm{MHz}, \mathrm{CDCl}_{3}\right) \delta 196.7,134.3,133.9,128.9(2 \mathrm{C})$, 128.7 (2C), 58.4, 16.5. NMR data correspond to the reported values. ${ }^{27}$

\section{2-Azido-1-(4-bromophenyl)propan-1-one (5i)}<smiles>CC(N)C(=O)c1ccc(Br)cc1</smiles>

$5 \mathbf{i}$

Starting from $(E)-((1-(4-b r o m o p h e n y l) p r o p-1-e n-1-y l) o x y) t r i m e t h y l s i l a n e$ (4i) (0.114 g, $0.400 \mathrm{mmol}), 2$-azido-1-(4-bromophenyl)propan-1-one (5i) $(0.0833 \mathrm{~g}, 0.328 \mathrm{mmol}, 82 \%$ yield $)$ was obtained as a yellow oil. $\mathrm{R}_{\mathrm{f}}: 0.3$ (Pentane/DCM 7:3). ${ }^{1} \mathbf{H}$ NMR (400 MHz, $\left.\mathrm{CDCl}_{3}\right) \delta 7.80$ (m, $\left.2 \mathrm{H}, \mathrm{CHAr}\right)$, 7.63 (m, $2 \mathrm{H}, \mathrm{CHAr}), 4.63$ (q, $1 \mathrm{H}, J=7.0 \mathrm{~Hz}, \mathrm{CHAr}), 1.55$ (d, $3 \mathrm{H}, J=$ $\left.7.0 \mathrm{~Hz}, \mathrm{CH}_{3}\right) .{ }^{13} \mathbf{C}$ NMR $\left(101 \mathrm{MHz}, \mathrm{CDCl}_{3}\right) \delta 195.7,133.0,132.3$ (2C), $130.2(2 \mathrm{C}), 129.3,58.4$, 16.3. IR $v_{\max } 2988(\mathrm{w}), 2987(\mathrm{w}), 2118(\mathrm{~m}), 2102(\mathrm{~m}), 1690(\mathrm{~m}), 1569(\mathrm{w}), 1568(\mathrm{w}), 1486(\mathrm{w})$, 1452 (w), 1399 (w), $1379(\mathrm{w}), 1357$ (w), $1277(\mathrm{w}), 1277$ (w), $1251(\mathrm{w}), 1213$ (m), $1181(\mathrm{w})$, 
1115 (w), 1102 (w), 1101 (w), 1073 (m), 964 (m), 911 (m), 840 (m), 777 (w), 733 (s). HRMS (APPI) calcd for $\mathrm{C}_{9} \mathrm{H}_{8}{ }^{79} \mathrm{BrNO}\left[\mathrm{M}-\mathrm{N}_{2}\right]^{+} 224.9784$; found 224.9795 .

\section{2-Azido-1-(4-(trifluoromethyl)phenyl)propan-1-one (5j)}<smiles>CC(N)C(=O)c1ccc(C(F)(F)F)cc1</smiles>

$5 \mathbf{j}$

Starting from (E)-trimethyl((1-(4-(trifluoromethyl)phenyl)prop-1-en-1yl)oxy)silane (4j) $\quad(0.106 \quad \mathrm{~g}, \quad 0.386 \quad \mathrm{mmol}), \quad$ 2-azido-1-(4(trifluoromethyl)phenyl)propan-1-one (5j) (0.0703 g, $0.289 \mathrm{mmol}, 75 \%$ yield) was obtained as a yellow oil. Rf: 0.3 (Pentane/DCM 3:2). ${ }^{\mathbf{1}} \mathbf{H}$ NMR $\left(400 \mathrm{MHz}, \mathrm{CDCl}_{3}\right) \delta 8.05(\mathrm{~d}, 2 \mathrm{H}, J=8.2 \mathrm{~Hz}, \mathrm{CHAr}), 7.76(\mathrm{~d}, 2 \mathrm{H}, J=$ $8.4 \mathrm{~Hz}, \mathrm{CHAr}), 4.70$ (q, $1 \mathrm{H}, J=7.0 \mathrm{~Hz}, \mathrm{CH}), 1.58$ (d, $\left.3 \mathrm{H}, J=7.0 \mathrm{~Hz}, \mathrm{CH}_{3}\right) .{ }^{\mathbf{1 3}} \mathbf{C} \mathbf{N M R}(101$ $\left.\mathrm{MHz} \mathrm{CDCl}_{3}\right) \delta 195.8,137.1,135.1\left(\mathrm{q}, J_{C-F}=33 \mathrm{~Hz}\right), 129.1(2 \mathrm{C}), 126.0\left(\mathrm{q}, J_{C-F}=4 \mathrm{~Hz}, 2 \mathrm{C}\right)$, $123.4\left(\mathrm{q}, J_{C-F}=273 \mathrm{~Hz}\right), 58.7,16.0$. IR $v_{\max } 2990(\mathrm{w}), 2121(\mathrm{~m}), 2106(\mathrm{~m}), 1789(\mathrm{w}), 1770(\mathrm{w})$, 1699 (m), 1581 (w), 1513 (w), 1452 (w), 1381 (w), 1324 (s), 1251 (w), 1217 (m), 1171 (m), 1131 (s), 1068 (s), 1018 (m), 1000 (w), 967 (m), 913 (w), 855 (m), 791 (w), 767 (w), 739 (w), 707 (w). HRMS (ESI) calcd for $\mathrm{C}_{10} \mathrm{~F}_{3} \mathrm{H}_{9} \mathrm{NO}^{+}\left[\mathrm{M}-\mathrm{N}_{2}+\mathrm{H}\right]^{+} 216.0631$; found 216.0642.

\section{2-Azido-1-(thiophen-2-yl)propan-1-one (5k)}

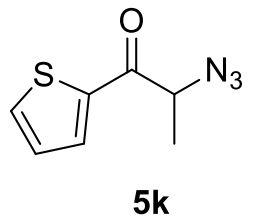

Starting from (E)-trimethyl((1-(thiophen-2-yl)prop-1-en-1-yl)oxy)silane (4k) (0.0868 g, 0.409 mmol), 2-azido-1-(thiophen-2-yl)propan-1-one (5k) (0.060 g, $0.33 \mathrm{mmol}, 81 \%$ yield) was obtained as a yellow oil. $\mathrm{R}_{\mathrm{f}}$ : 0.3 (Pentane/DCM 6:4). ${ }^{1} \mathbf{H}$ NMR $\left(400 \mathrm{MHz}, \mathrm{CDCl}_{3}\right) \delta 7.79(\mathrm{dd}, 1 \mathrm{H}, J=4.0,0.9 \mathrm{~Hz}, \mathrm{CHAr}), 7.73$ (dd, $1 \mathrm{H}, J=4.7,0.8 \mathrm{~Hz}, \mathrm{CHAr}), 7.17$ (dd, $1 \mathrm{H}, J=4.9,4.0 \mathrm{~Hz}, \mathrm{CHAr}$ ), 4.52 (q, $1 \mathrm{H}, J=7.0 \mathrm{~Hz}, \mathrm{CH}), 1.60\left(\mathrm{~d}, 3 \mathrm{H}, J=7.0 \mathrm{~Hz}, \mathrm{CH}_{3}\right) .{ }^{13} \mathbf{C} \mathbf{N M R}\left(101 \mathrm{MHz}, \mathrm{CDCl}_{3}\right) \delta 189.7$, 140.9, 135.2, 133.2, 128.5, 59.8, 17.0. IR $v_{\max } 3102(\mathrm{w}), 3093(\mathrm{w}), 2989(\mathrm{w}), 2109(\mathrm{~s}), 1670(\mathrm{~s})$, 1518 (w), 1450 (w), 1414 (s), 1378 (w), 1357 (w), 1323 (w), 1239 (s), 1223 (s), 1059 (m), 1043 (w), $1013(\mathrm{w}), 999(\mathrm{w}), 917$ (w), $862(\mathrm{w}), 848$ (w), 832 (w), 777 (w), 730 (s). HRMS (ESI) calcd for $\mathrm{C}_{7} \mathrm{H}_{8} \mathrm{NOS}^{+}\left[\mathrm{M}-\mathrm{N}_{2}+\mathrm{H}\right]^{+} 154.0321$; found 154.0325 . 


\section{Enantioselective azidation of $\beta$-keto esters}

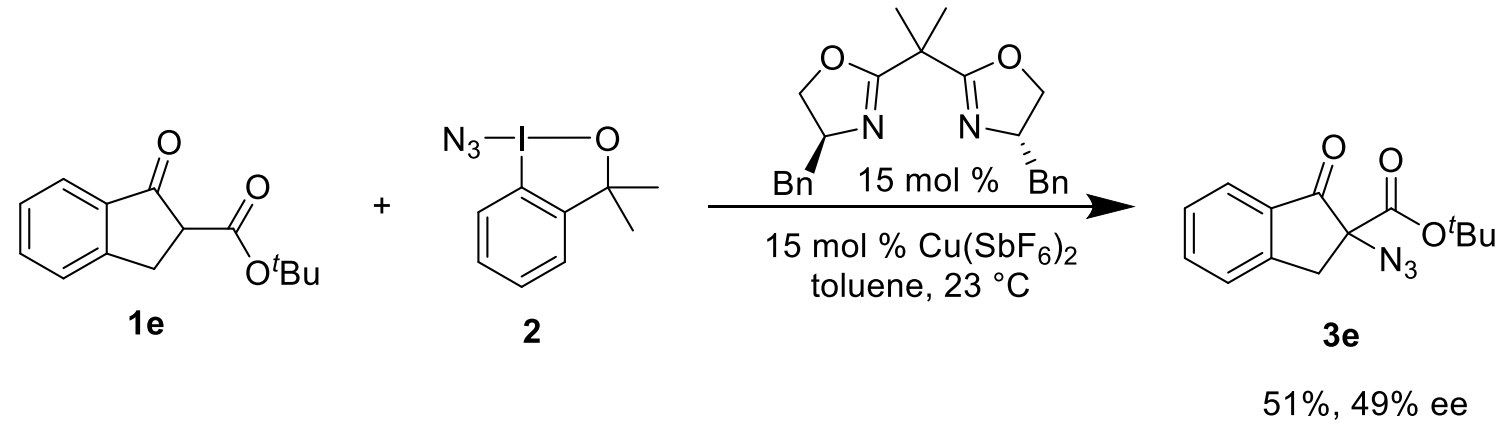

Preparation of the complex.

$\mathrm{CuCl}_{2}$ (2.0 mg, $0.015 \mathrm{mmol}, 1.0$ equiv) and (+)-2,2'-Isopropylidenebis[(4R)-4-benzyl-2oxazoline] (5.3 mg, $0.015 \mathrm{mmol}, 1.0$ equiv) were stirred in a microwave tube of $2 \mathrm{~mL}$ in $\mathrm{CH}_{2} \mathrm{Cl}_{2}$ $(0.5 \mathrm{~mL})$ for $2 \mathrm{~h}$. Then the solution was transferred into another microwave tube where $\mathrm{AgSbF}_{6}$ (10 mg, $0.030 \mathrm{mmol}, 2.05$ equiv) was previously placed. The reaction was stirred at room temperature for $5 \mathrm{~h}$. The mixture was filtered and the solvent evaporated. The resulting complex was stored in a dry box and used as soon as possible.

\section{Enantioselective protocol.}

A solution of reagent $2(15 \mathrm{mg}, 0.055 \mathrm{mmol}, 1.3$ equiv) in toluene $(0.43 \mathrm{~mL}, 0.1 \mathrm{M}$ in 1e) was added in a microwave tube of $2 \mathrm{~mL}$ to the preformed complex $\left(5.7 \mathrm{mg}, 6.010^{-3} \mathrm{mmol}, 0.014\right.$ equiv) and the starting material 1e $(10 \mathrm{mg}, 0.043 \mathrm{mmol})$. The reaction was stirred at room temperature and followed by TLC (using the solvent indicated for the $\mathrm{R}_{\mathrm{f}}$ value, visualization with $\mathrm{UV}$ or $p$-anisaldehyde stain) until disappearance of the starting material.

The solvent was removed by evaporation and the crude product was purified by flash chromatography (DCM/pentane 4:1) and product 3e (0.056 g, $0.21 \mathrm{mmol}, 51 \%$ yield).

A small sample of pure compound was analyzed by chiral HPLC: (IC, Hexane/ $/ \mathrm{PrOH}=99 / 1,1$ $\mathrm{mL} / \mathrm{min}, 254 \mathrm{~nm}), \mathrm{tr}($ major isomer $)=11.8 \mathrm{~min}, \mathrm{tr}($ minor isomer $)=13.3 \mathrm{~min}(49 \% \mathrm{ee})$. 
Racemic 3e

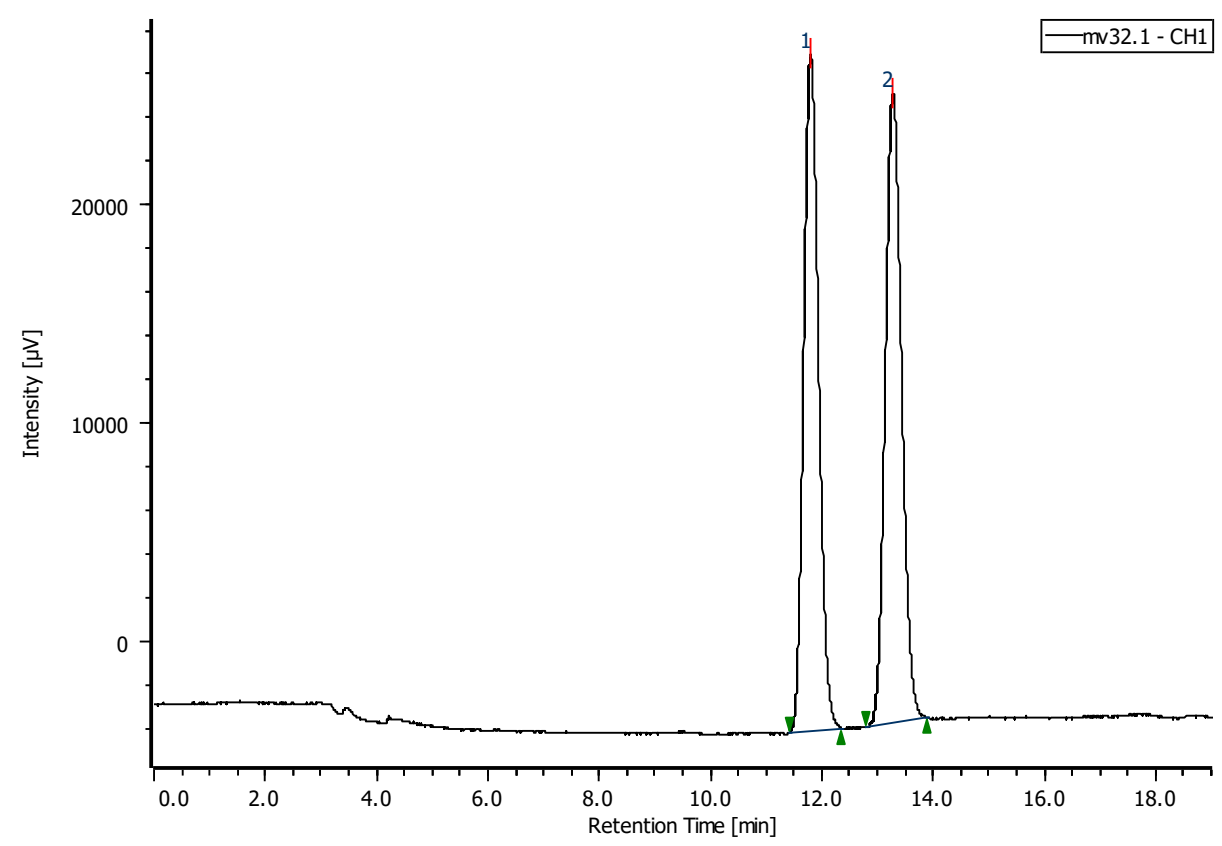

Enantioenriched 3e

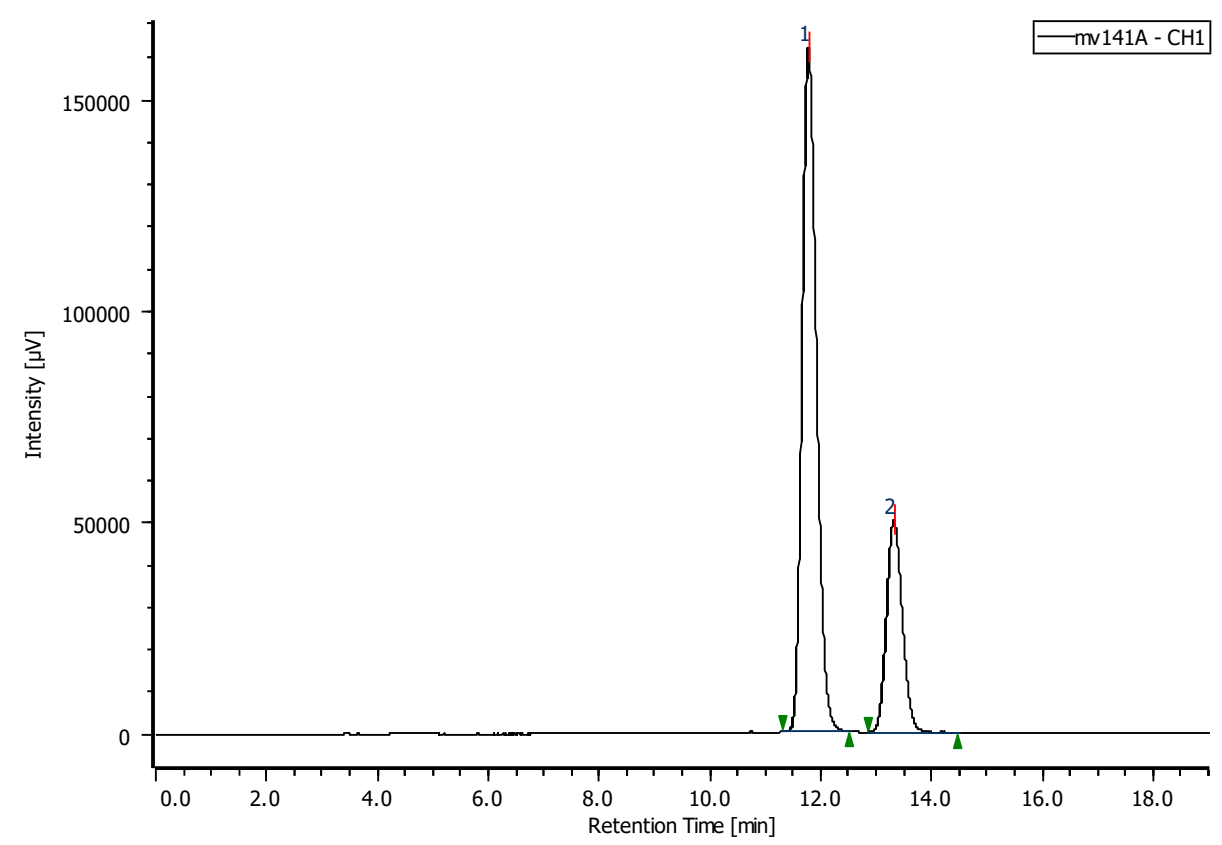




\section{Reference}

(1) Powers, D. C.; Lee, E.; Ariafard, A.; Sanford, M. S.; Yates, B. F.; Canty, A. J.; Ritter, T. J. Am. Chem. Soc. 2012, 134, 12002.

(2) Akai, S.; Okuno, T.; Egi, M.; Takada, T.; Tohma, H.; Kita, Y. Heterocycles 1996, 42, 47.

(3) Fernández González, D.; Brand, J. P.; Waser, J. Chem. Eur. J. 2010, 16, 9457.

(4) Eisenberger, P.; Gischig, S.; Togni, A. Chem. Eur. J. 2006, 12, 2579.

(5) Emelen, K. V.; De Wit, T.; Hoornaert, G. J.; Compernolle, F. Tetrahedron 2002, 58, 4225

(6) Kobayashi, S.; Gustafsson, T.; Shimizu, Y; Kiyohara, H.; Matsubara, R. Org. Lett. 2006, 8, 4923.

(7) Hale, K. J.; Grabski, M.; Flasz, J.T. Org. Lett. 2013, 15, 370.

(8) Moss, T. A.; Fenwick, D. R.; Dixon, D. J. J. Am. Chem. Soc. 2008, 130, 10076.

(9) Smith, A. M. R.; Rzepa, H. S.; White, A. J. P.; Billen, D.; Hii, K. K. J. Org. Chem. 2010, 75,3085 .

(10) Fernandez Gonzalez, D.; Waser, J.; Adv. Synth. Catal. 2013, DOI: 10.1002/adsc.20130026.

(11) Sato, K.; Isoda, M.; Ohata, S.; Morita, S.; Tarui, A.; Omote, M.; Kumadaki, I; Ando, A. Adv. Syn. Catal. 2012, 354, 510.

(12) Zhang, Z.; Liu, Y.; Gong, M.; Zhao, X.; Zhang, Y.; Wang, J. Angew. Chem., Int. Ed. 2010, 49, 1139.

(13) Poulsen, T. B.; Bernardi, L.; Aleman, J.; Overgaard, J.; Jorgensen, K. A. J. Am. Chem. Soc 2007, 129, 441.

(14) Jones, R. A.; Stokes, M. J. Tetrahedron 1984, 40, 1051.

(15) Sheridan, H.; Walsh, J J.; Cogan, C.; Jordan, M.; McCabe, T.; Passante, E.; Frankish, N. H. Bioorg. Med. Chem. Lett. 2009, 19, 5927.

(16) Aljarilla, A.; Cordoba, R.; Csaky, A. G.; Fernandez, I.; Ortiz, F. L.; Plumet, J.; Gomez, G. R., Eur. J. Org. Chem. 2006, 3969.

(17) Einaga, H.; Nojima, M.; Abe, M. J. Chem. Soc. Perkin Trans. 1 1999, 17, 2507.

(18) Luttikhedde, H. J. G; Leino, R.; Lehtonen, A.; Naesman, J. H. J. Organomet. Chem. 1998, $555,127$.

(19) Pouliot, M.; Renaud, P.; Studer, A.; Vogler, T.; Schenk, K., Angew. Chem. Int. Ed. 2009, 48, 6037.

(20) Cheon, C.H.; Kanno, O.; Toste, F. D. J. Am. Chem. Soc. 2011, 133, 13248.

(21) Eames, J.; Coumbarides, Gregory S.; Suggate, Michael J.; Weerasooriya, N., Eur. J. Org. Chem. 2003 , 4, $634-641$.

(22) Shibatomi, K.; Soga, Y.; Narayama, A.; Fujisawa, I.; Iwasa, S. J. Am. Chem. Soc. 2012, 134, 9836.

(23) Deng, Q-H.; Bleith, T.; Wadepohl, H.; Gade, L. H. J. Am. Chem. Soc. 2013, 135, 5356.

(24) Youn, S. W.; Kim, B. S.; Jagdale, A. R. J. Am. Chem. Soc. 2012, 134, 11308.

(25) Benati, L.; Nanni, D.; Sangiorgi, C.; Spagnolo, P. J. Org. Chem. 1999, 64, 7836. 
(26) Harschneck, T.; Hummel, S.; Kirsch, S. F.; Klahn, P. Chem. Eur. J. 2012, 18, 1187.

(27) Patonay, T.; Hoffman, R. V. J. Org. Chem. 1994, 59, 2902. 
10.Spectra ds $\left({ }^{1} \mathrm{HNMR},{ }^{13} \mathrm{CNMR}, \mathrm{IR}\right)$ 


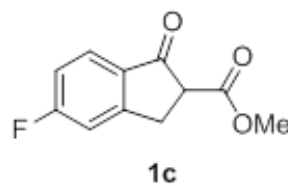

1c
${ }^{1} \mathrm{H}$ NMR

Solvent: $\mathbf{C D C l}_{3}$

Frequency: $400.08 \mathrm{MHz}$

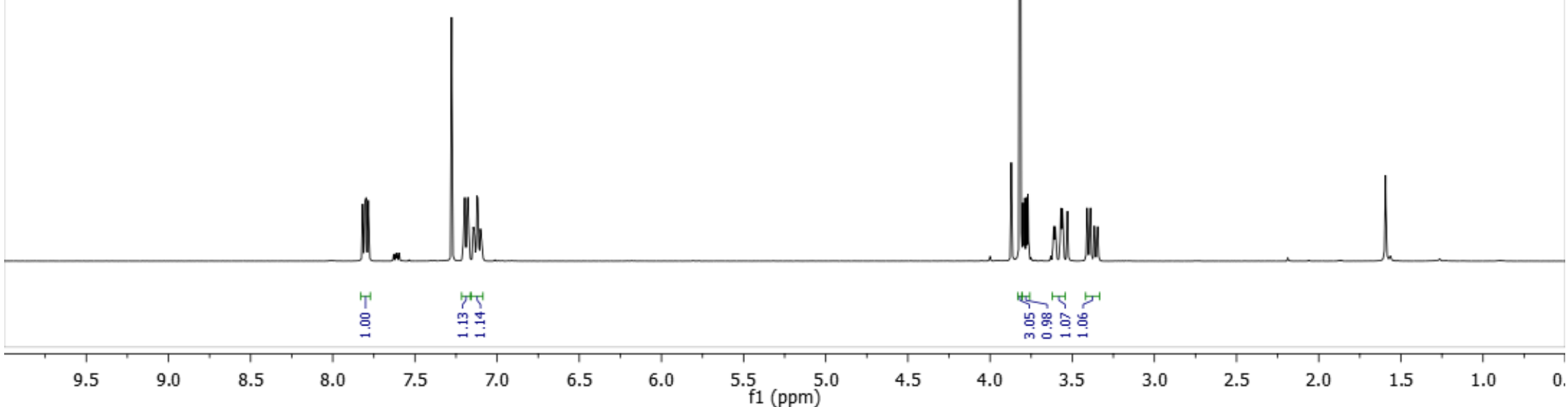




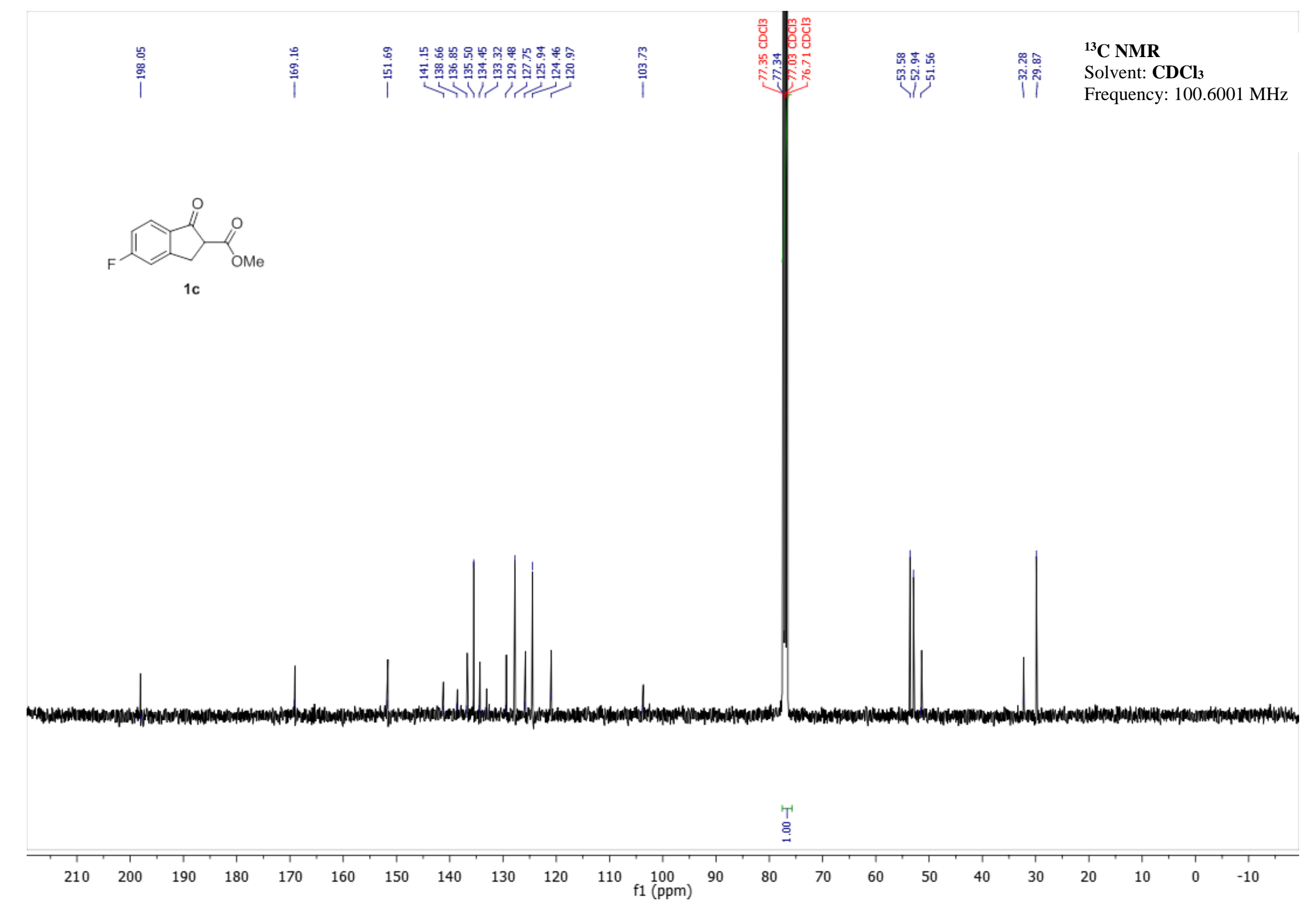




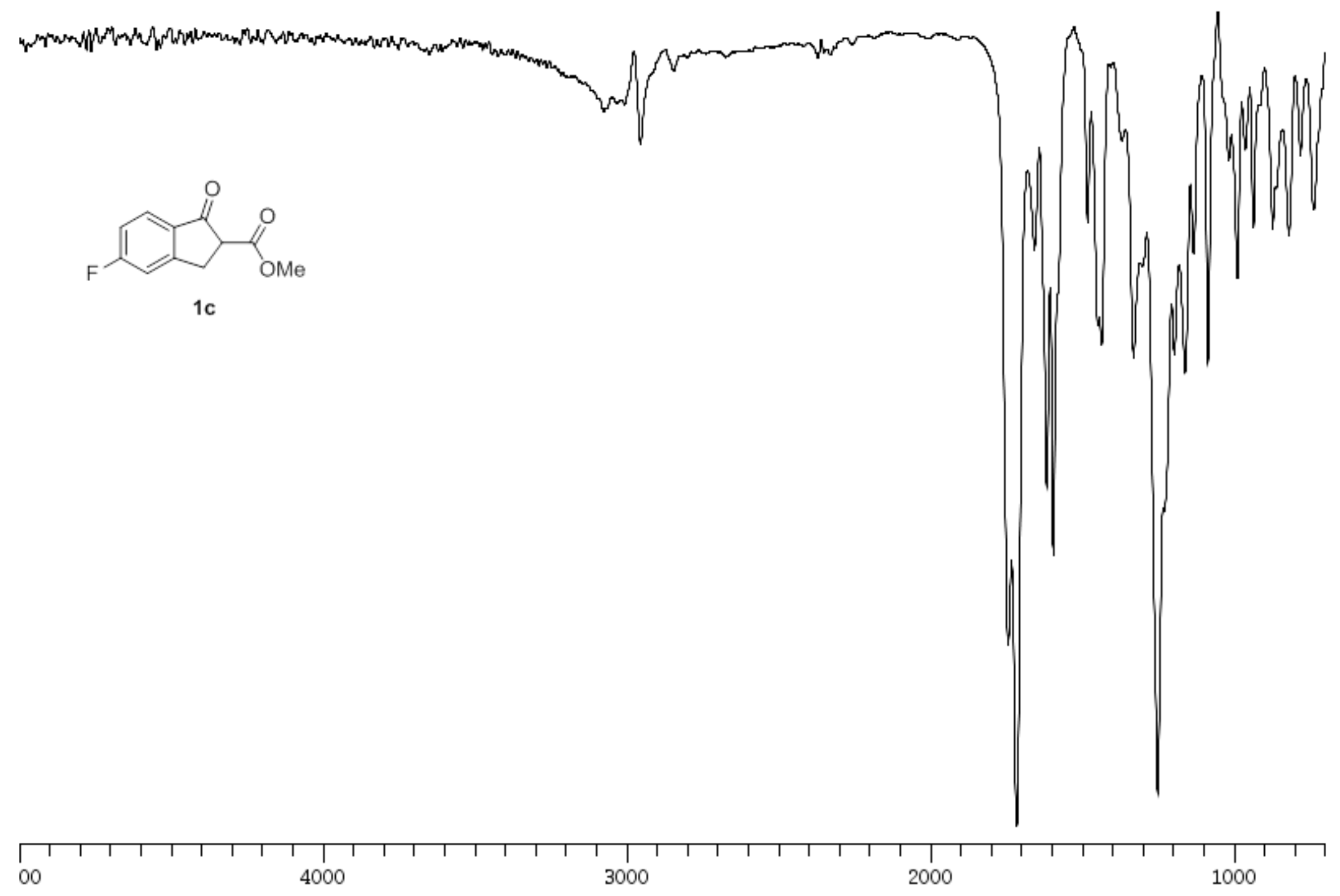




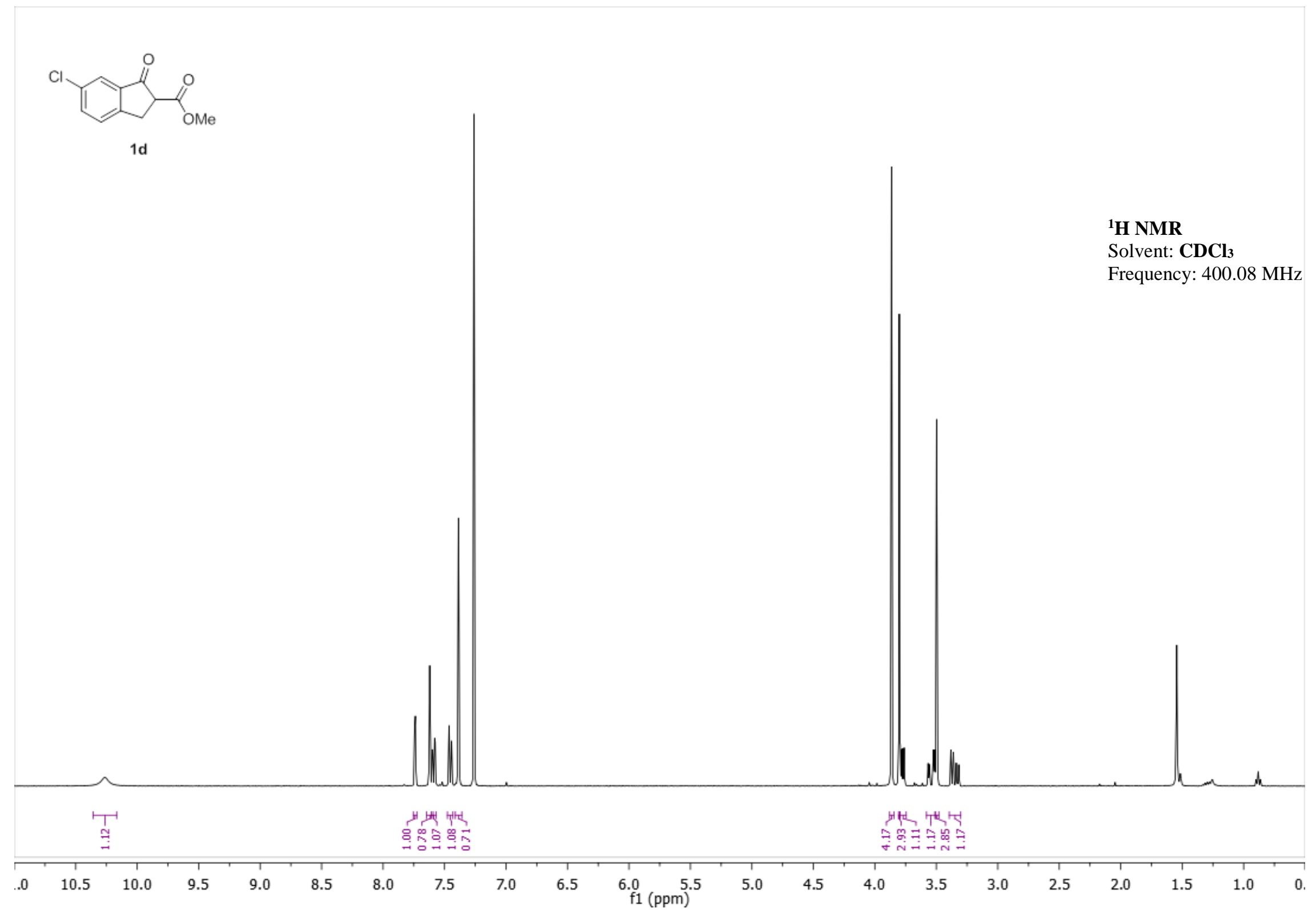

S34 


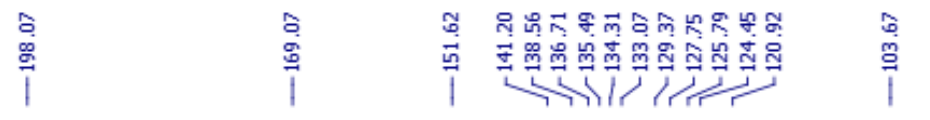

${ }^{13}$ C NMR

Solvent: $\mathbf{C D C l}_{3}$

Frequency: $100.6001 \mathrm{MHz}$

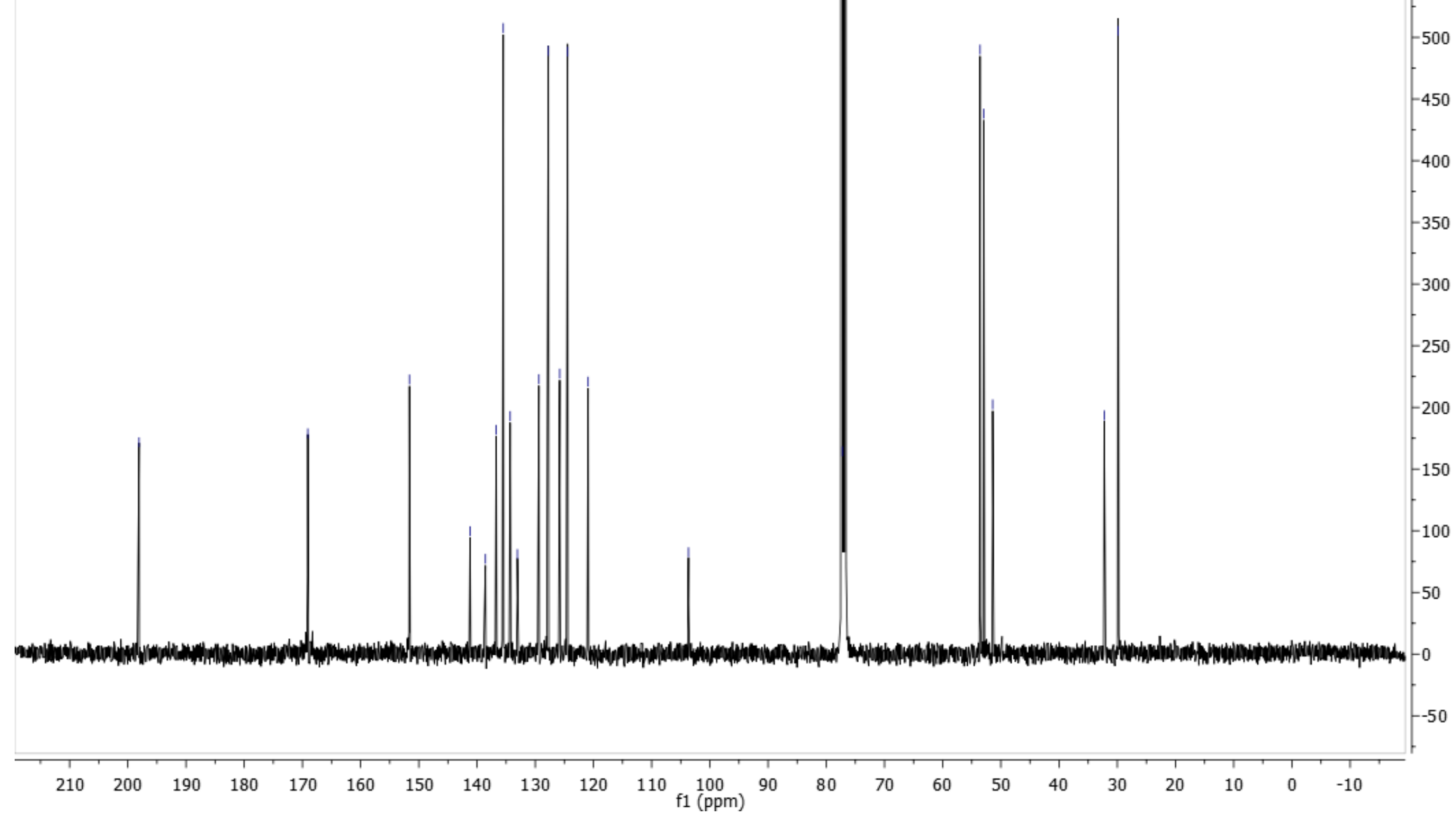




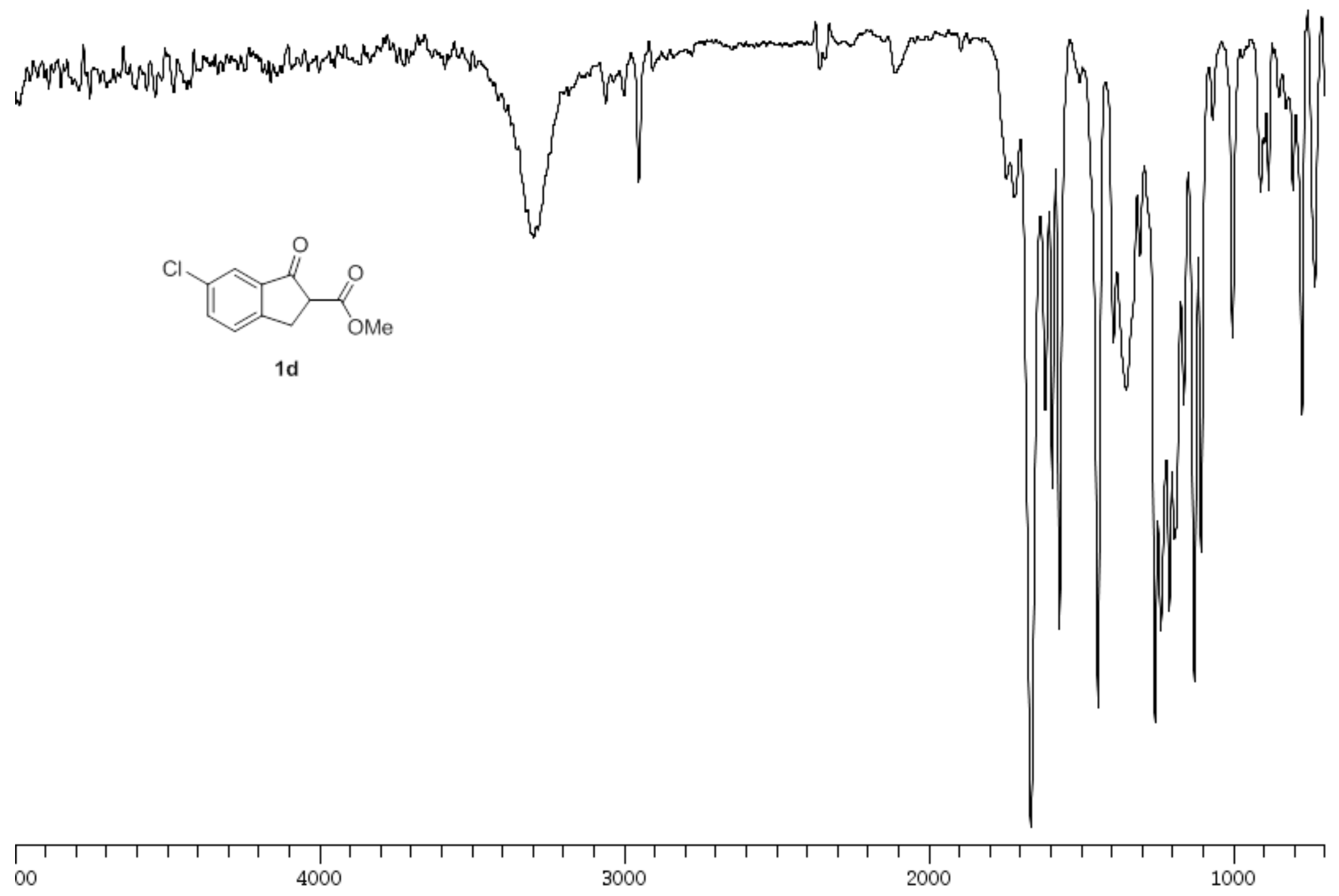

S36 

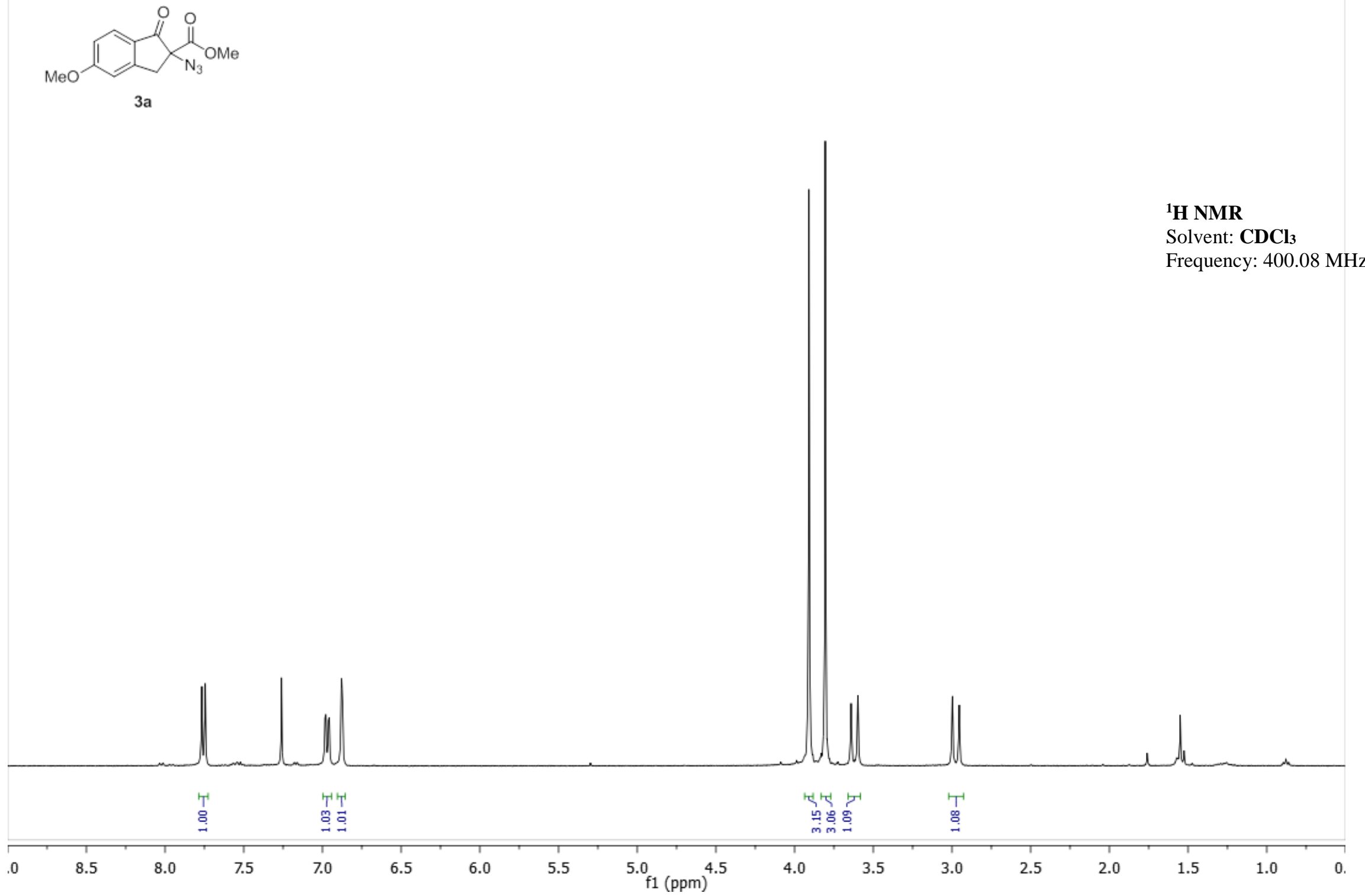

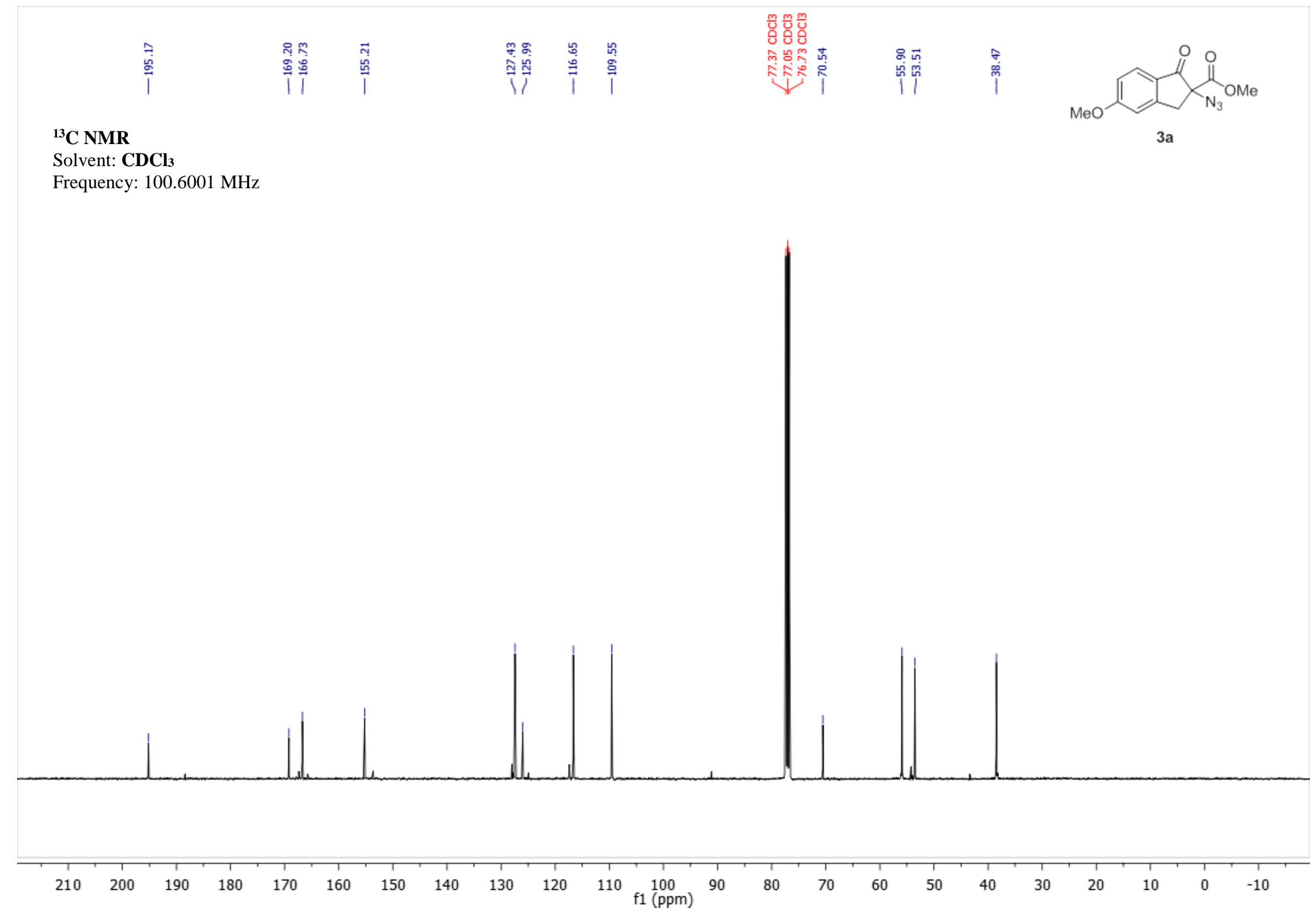


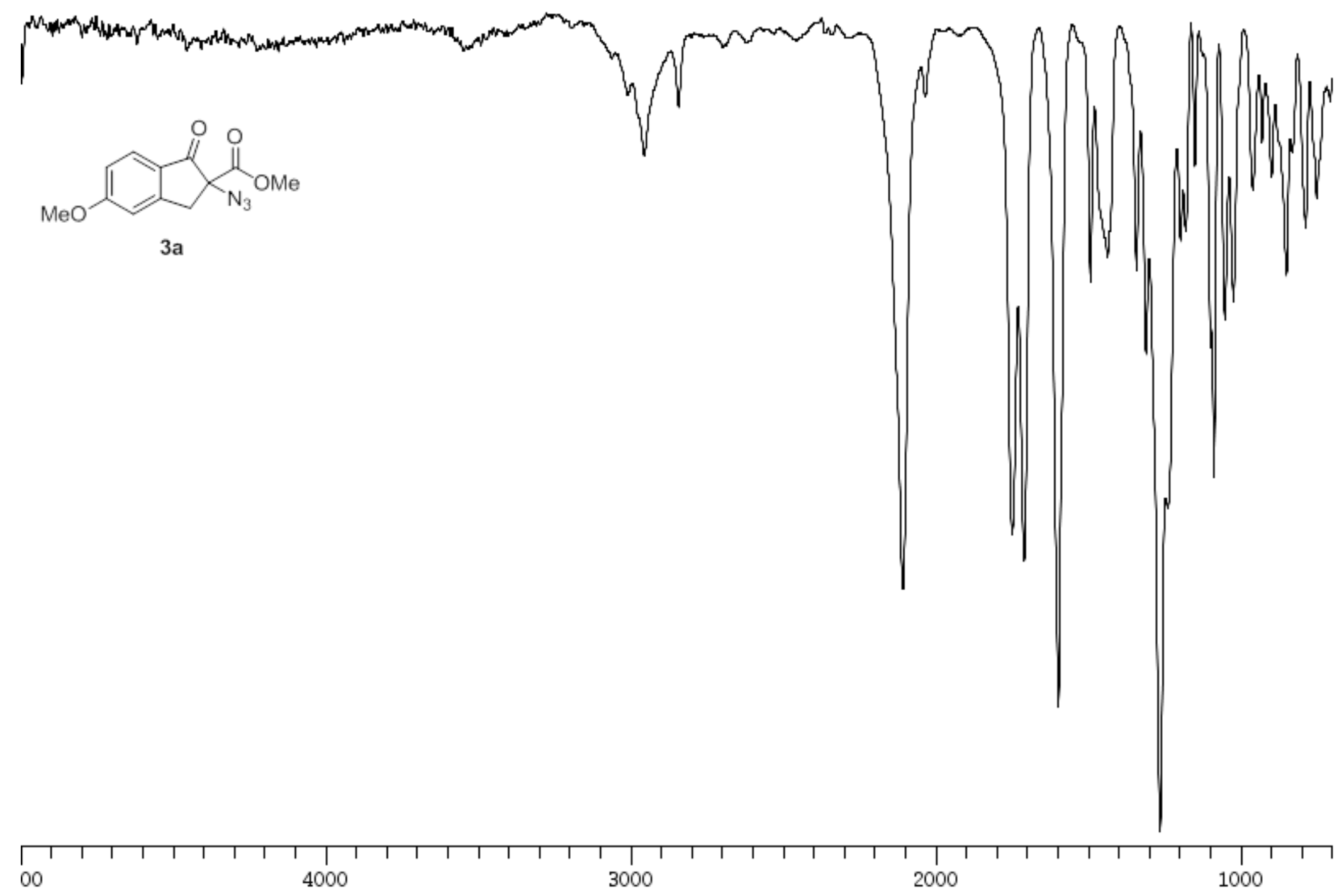




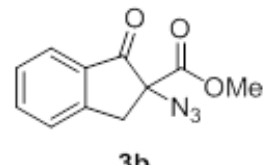

$3 \mathrm{~b}$

${ }^{1}$ H NMR

Solvent: $\mathbf{C D C l}_{3}$

Frequency: $400.08 \mathrm{MHz}$
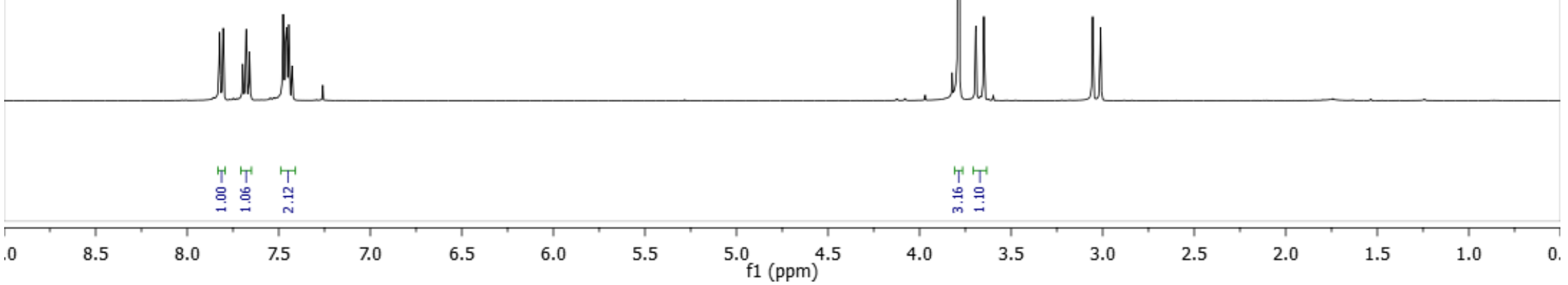

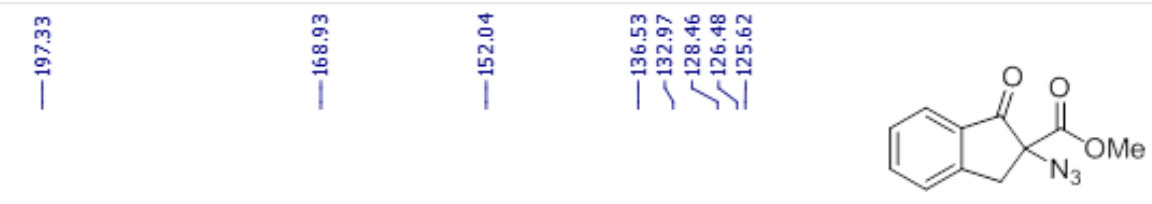

$\stackrel{9}{i}$ i

${ }^{13}$ C NMR

Solvent: $\mathbf{C D C l}_{3}$

Frequency: $100.6001 \mathrm{MHz}$

3b
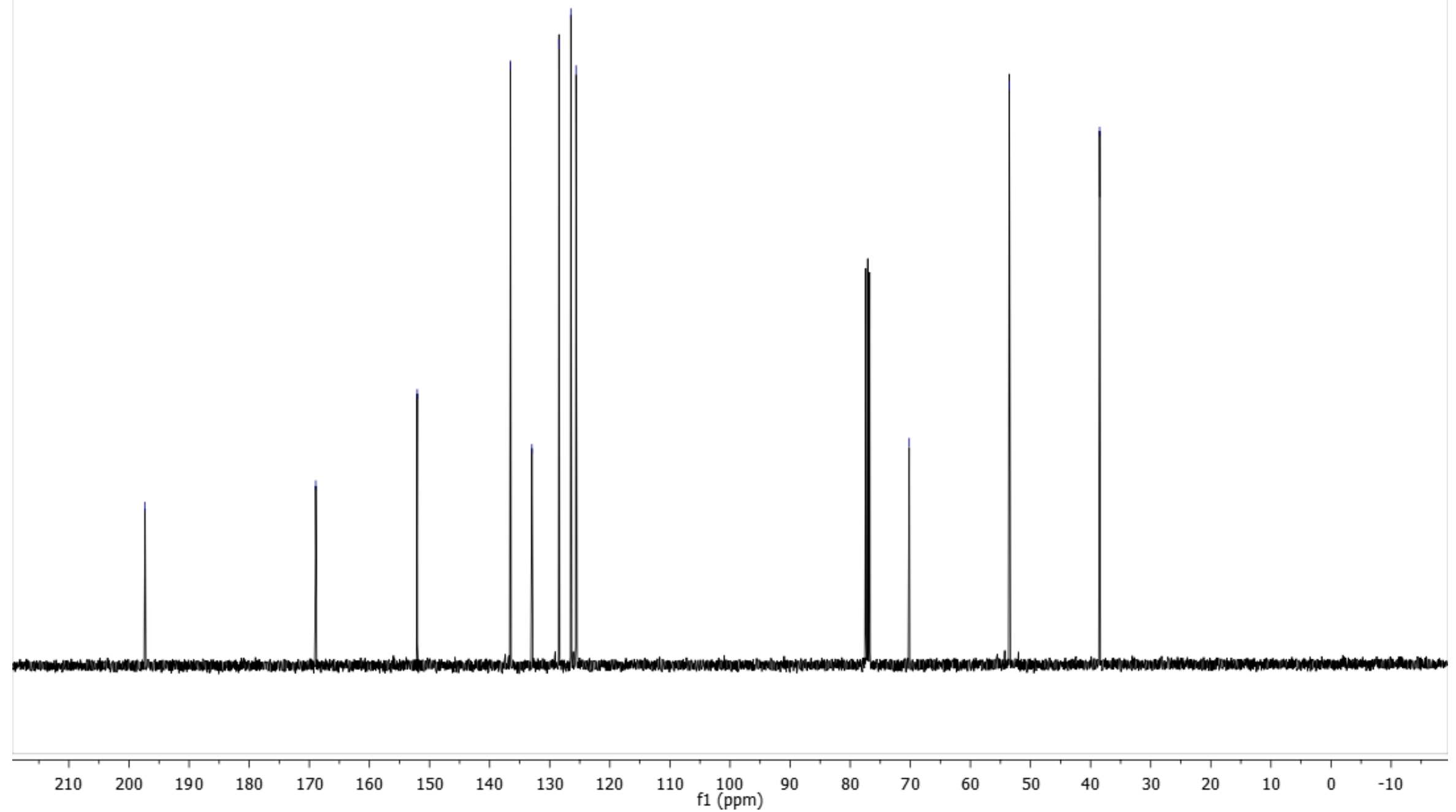


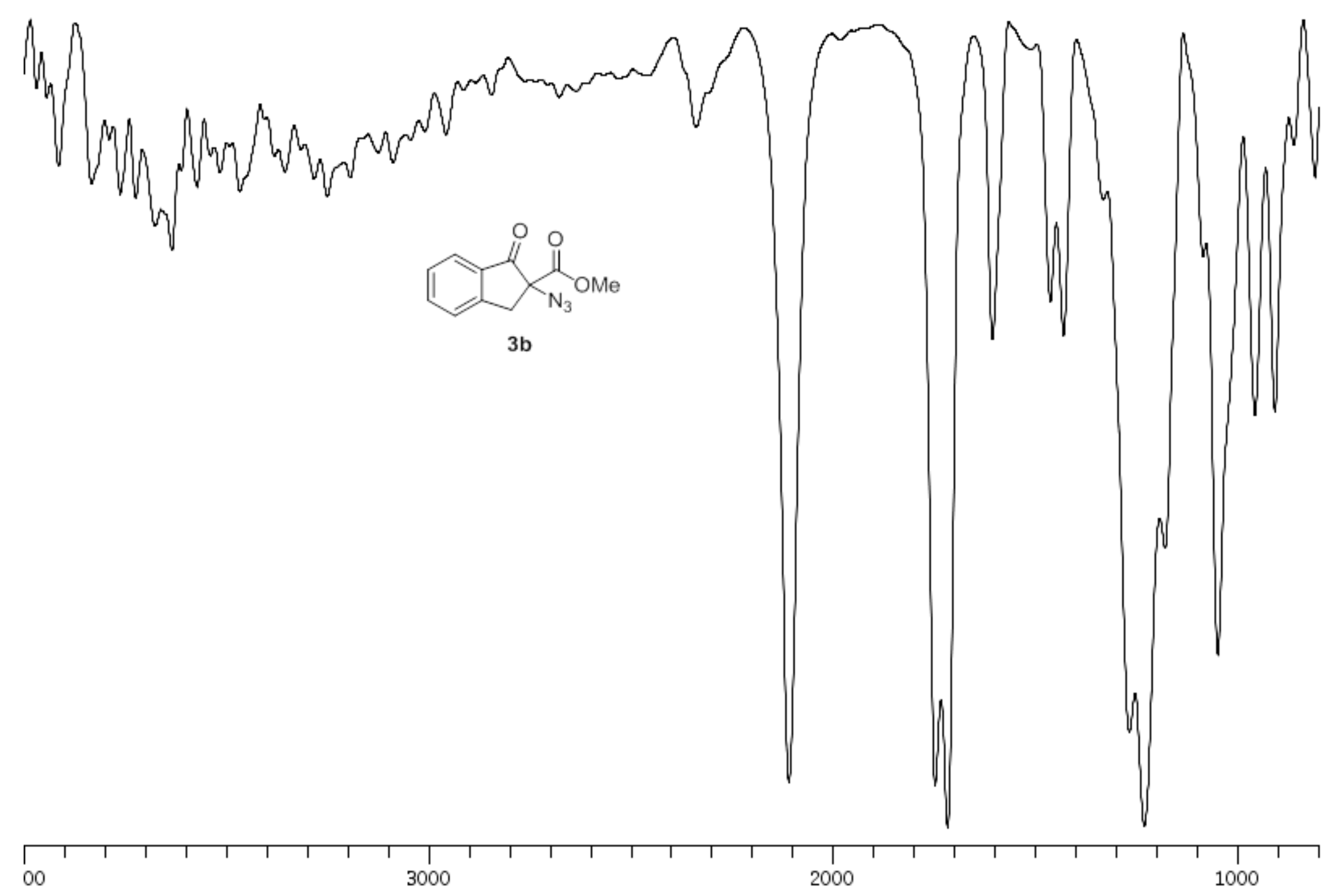




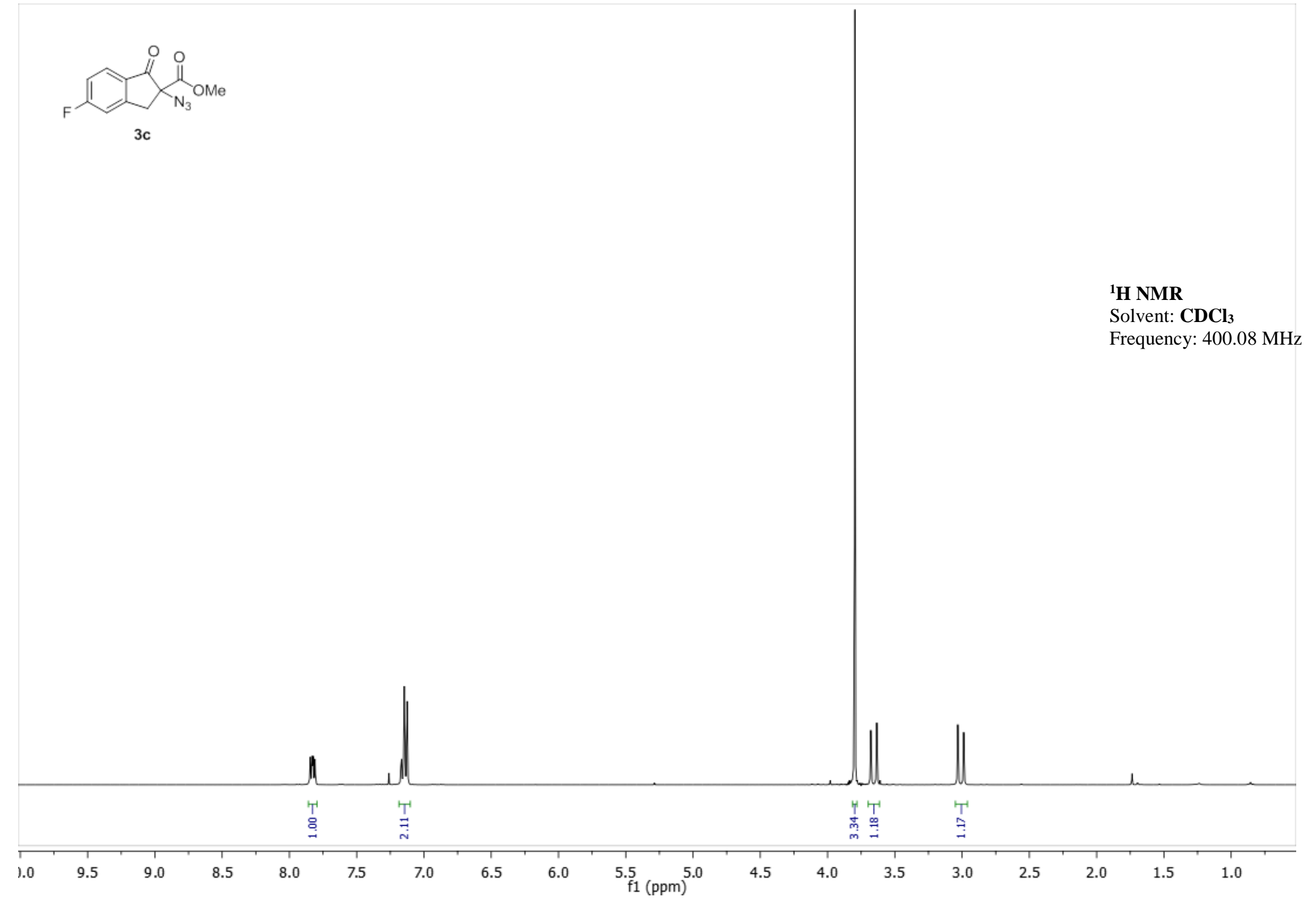



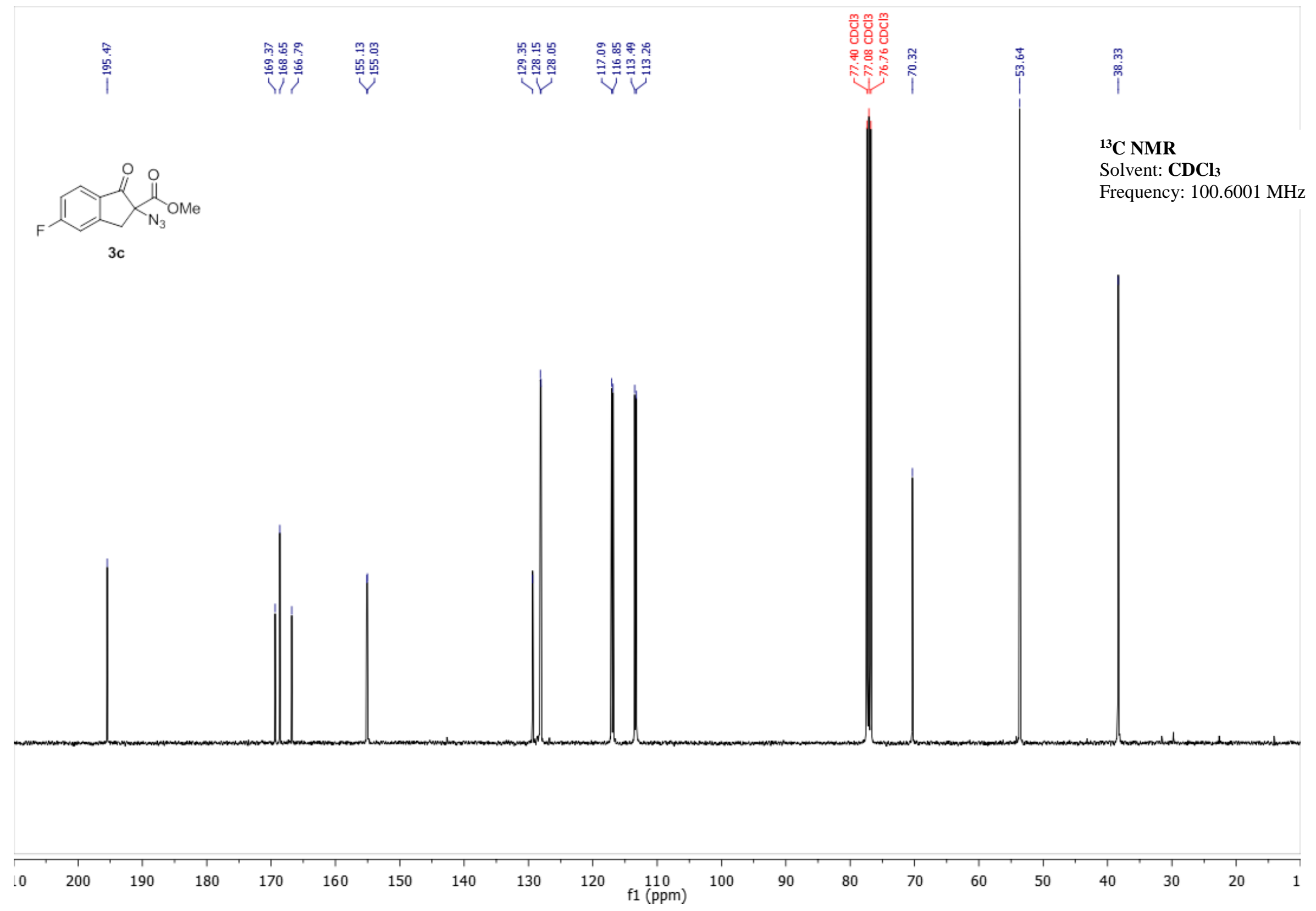


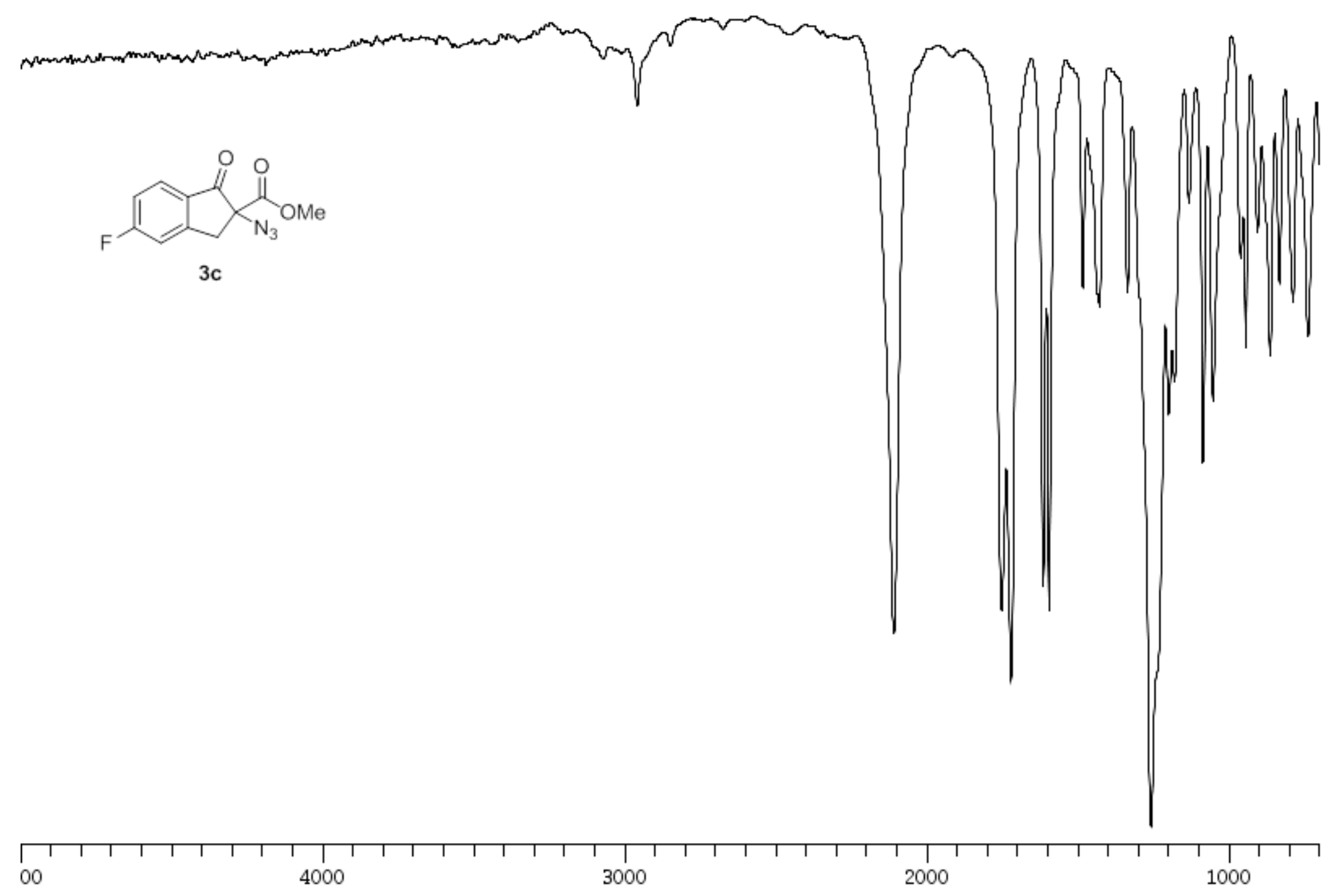



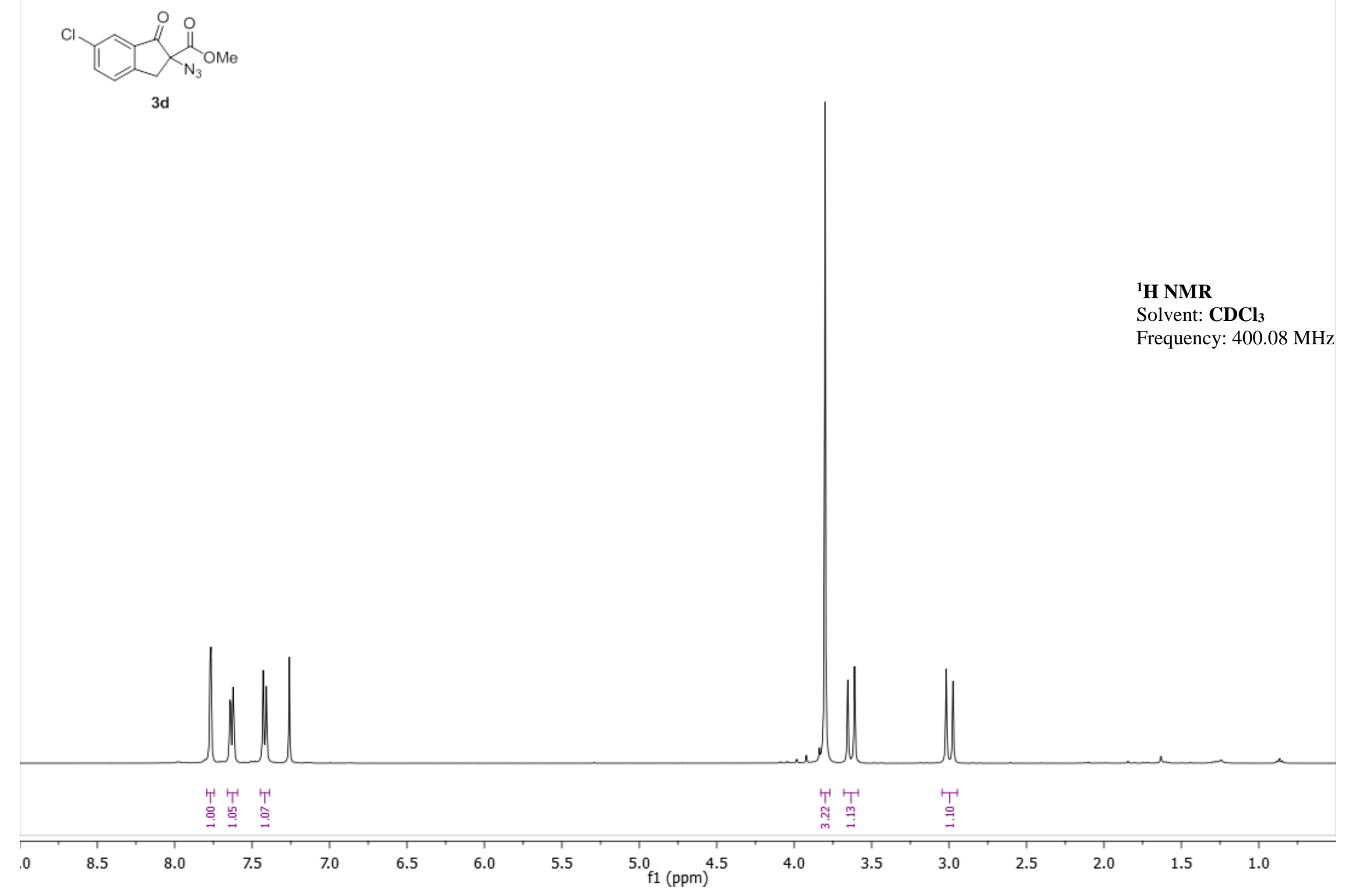

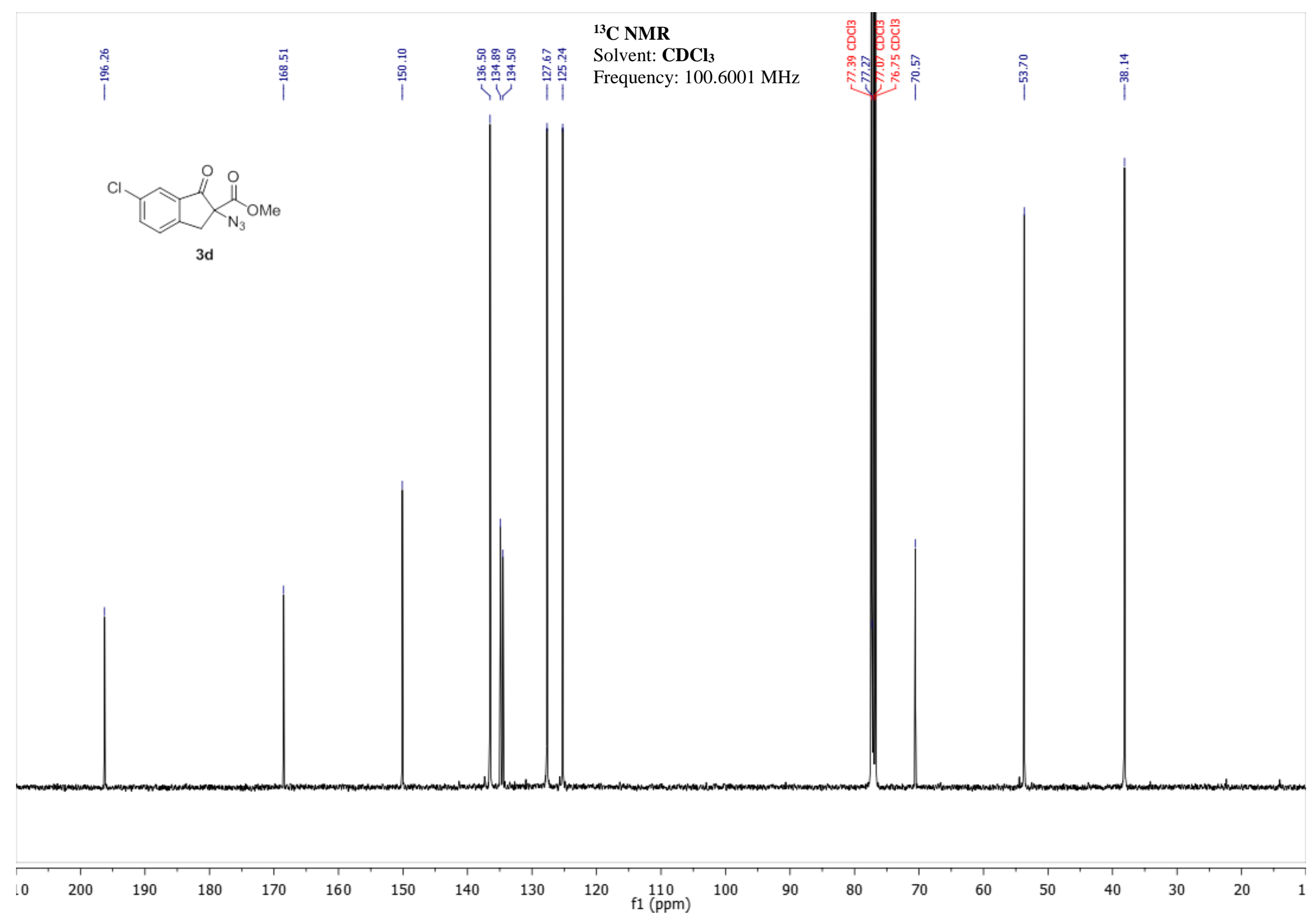


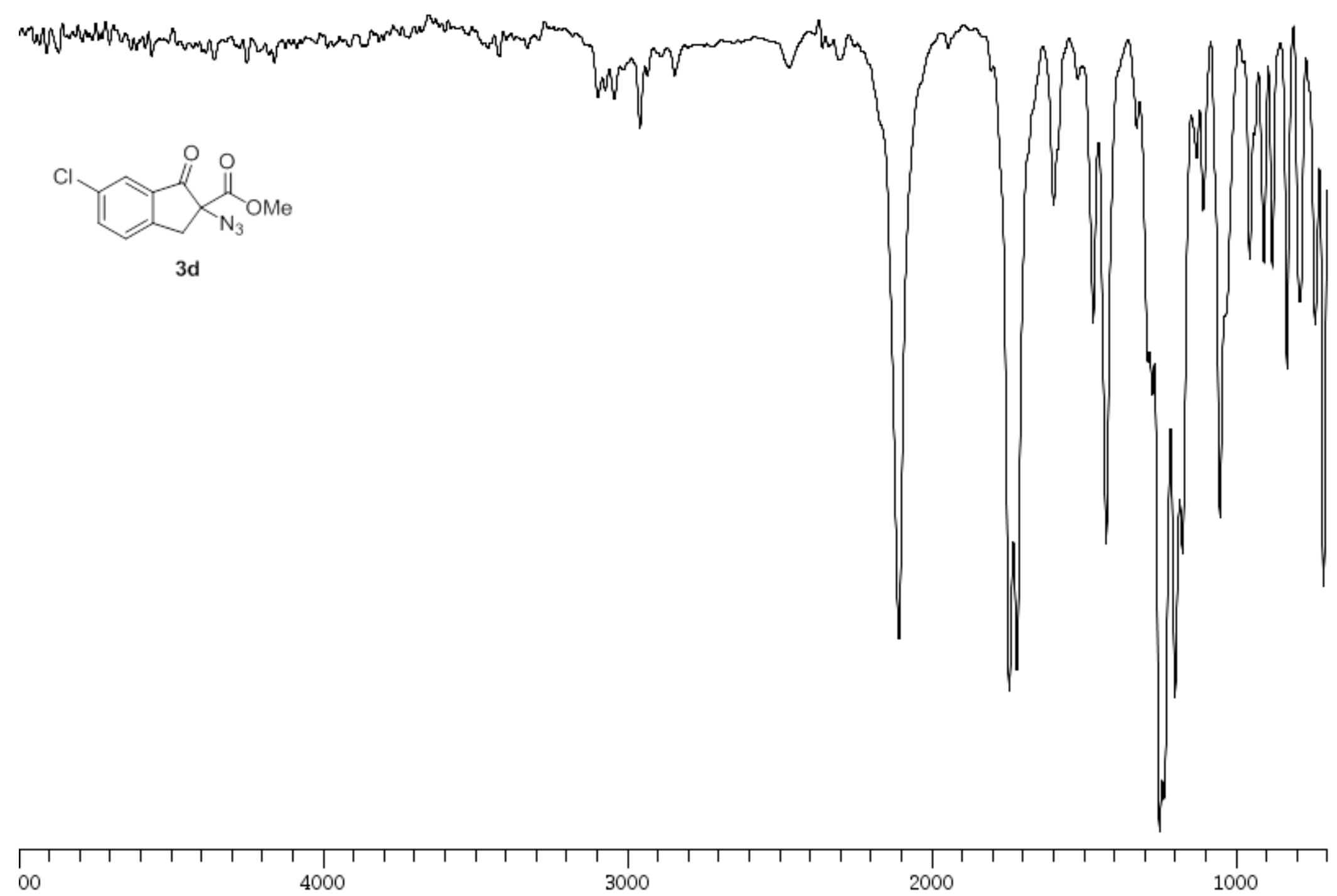




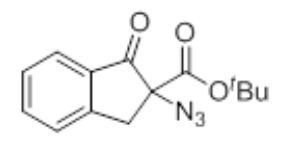

$3 e$

${ }^{1}$ H NMR

Solvent: $\mathbf{C D C l}_{\mathbf{3}}$

Frequency: $400.08 \mathrm{MHz}$

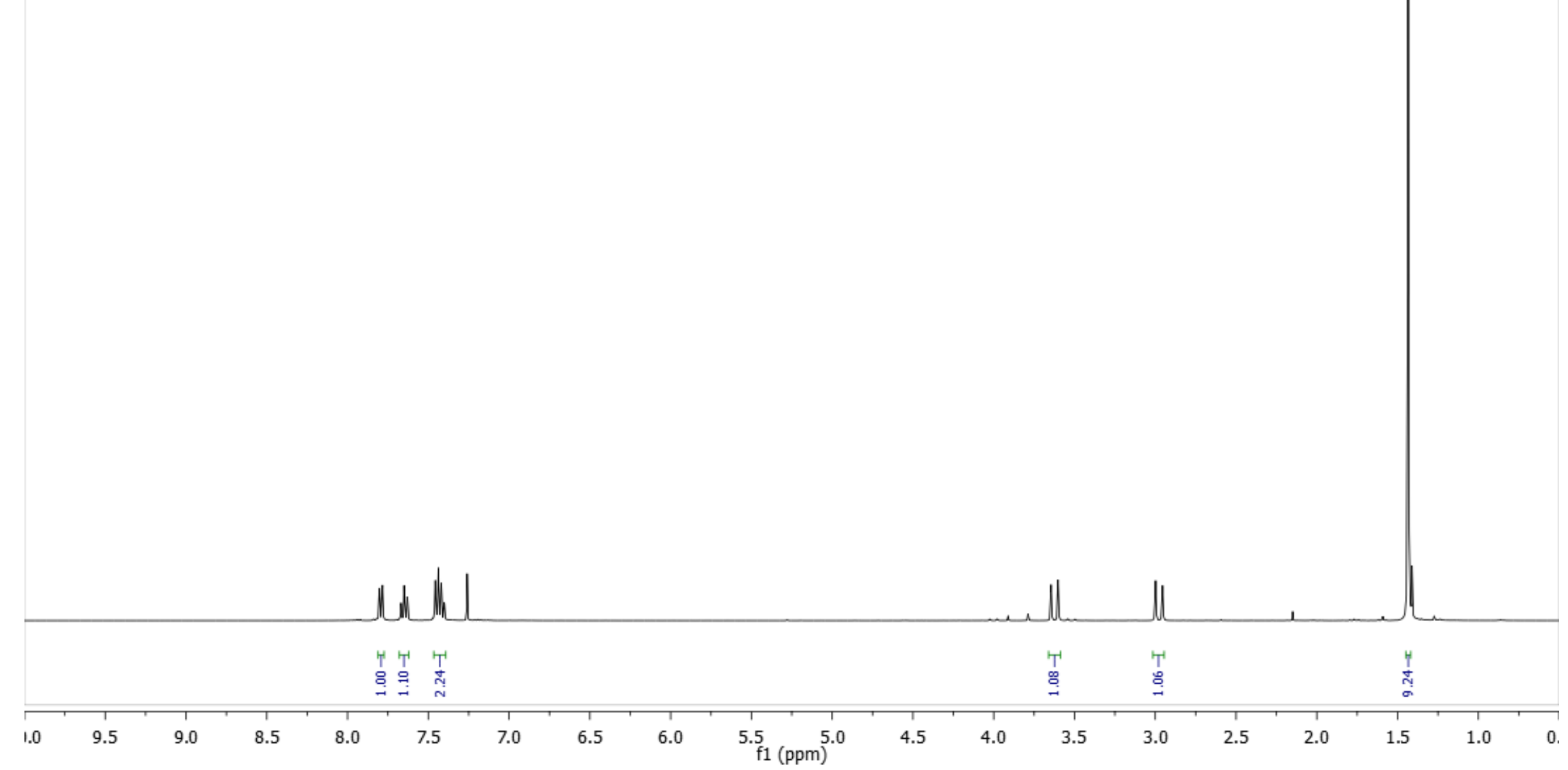




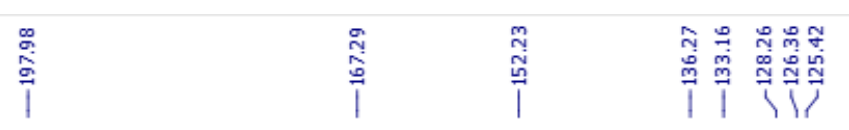

${ }^{13}$ C NMR

Solvent: $\mathbf{C D C l}_{\mathbf{3}}$

Frequency: $100.6001 \mathrm{MHz}$

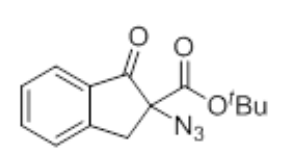

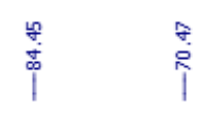

3e i

$\stackrel{m}{i}$

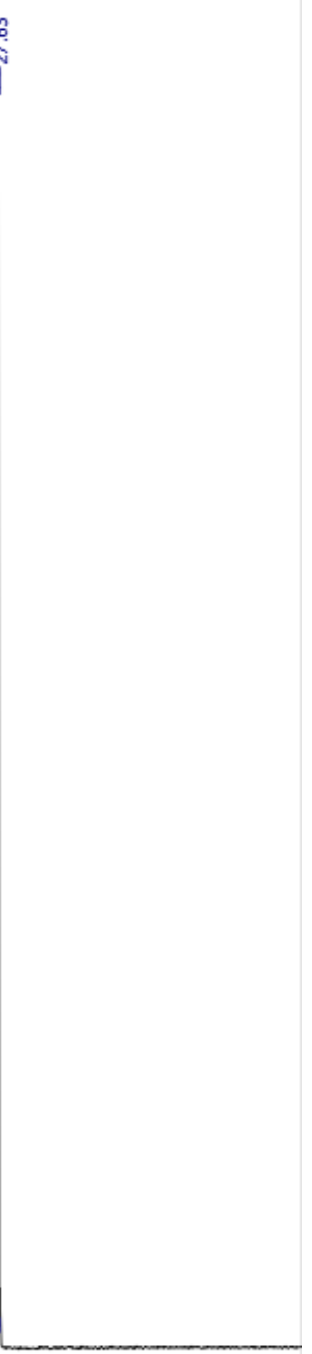

$10 \quad 200$

$200 \quad 190$

$190 \quad 180 \quad 170 \quad 160$ 


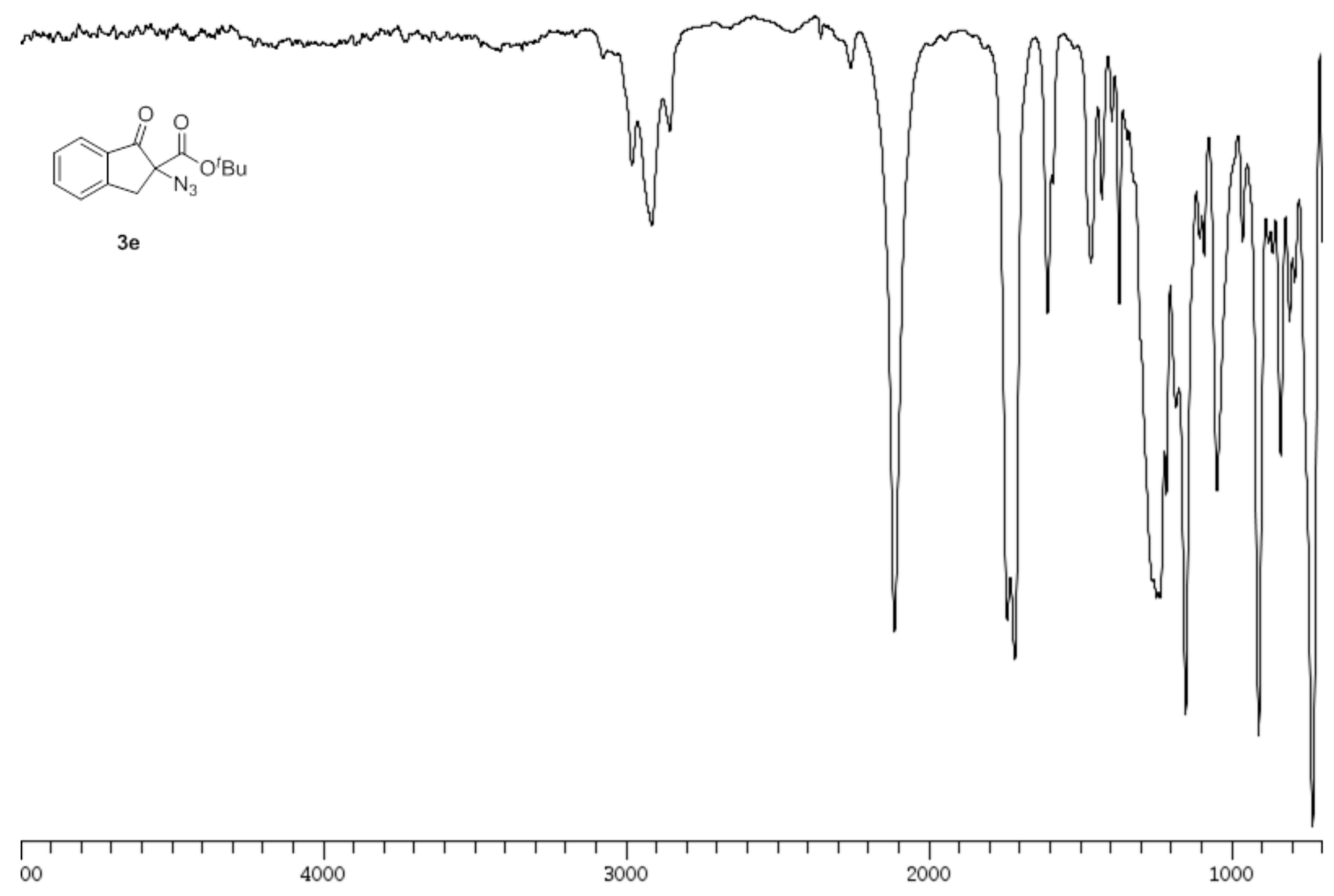




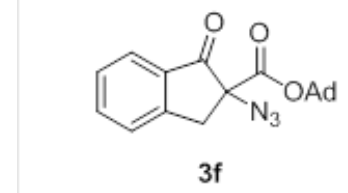

${ }^{1}$ H NMR

Solvent: $\mathbf{C D C l}_{3}$

Frequency: $400.08 \mathrm{MHz}$

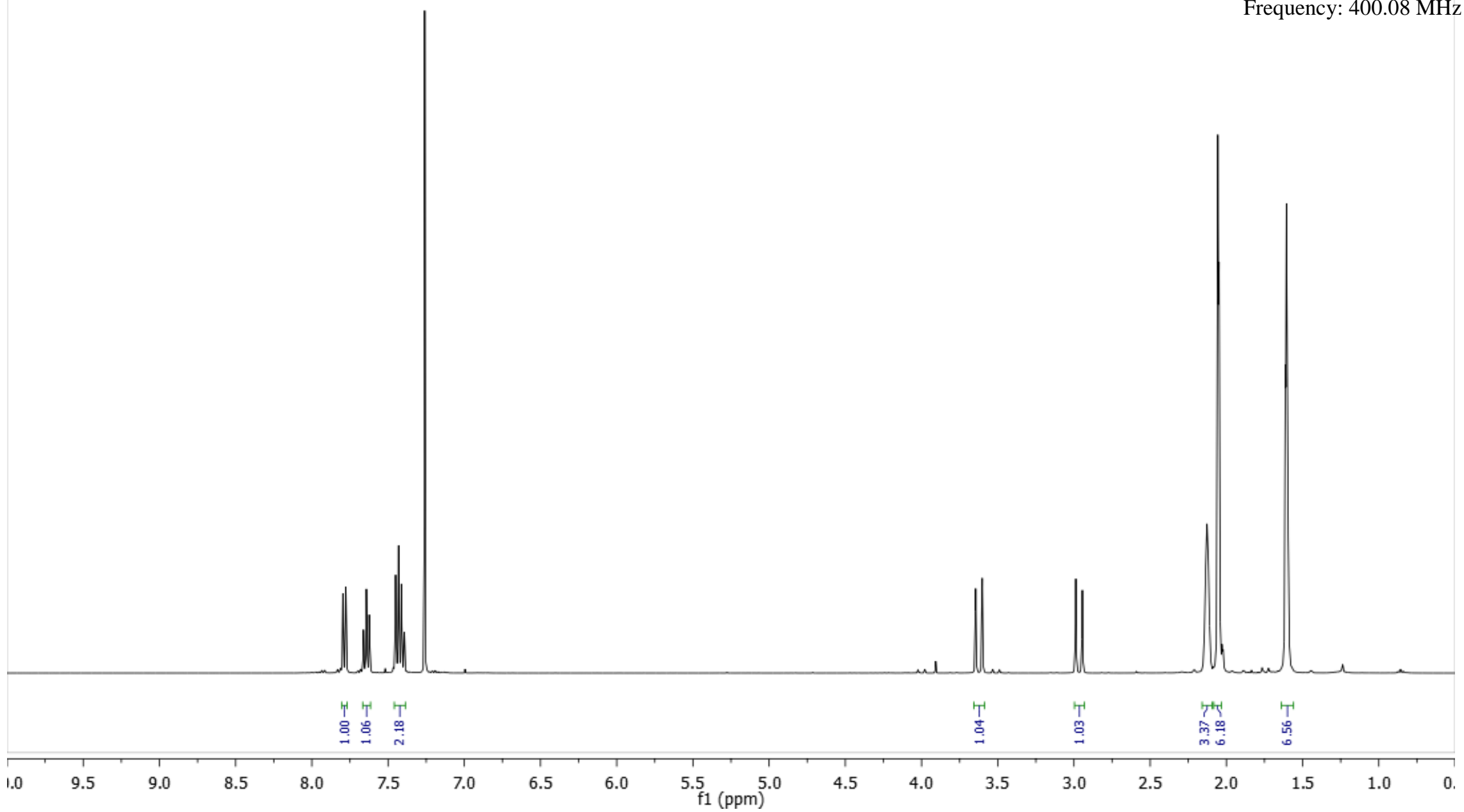




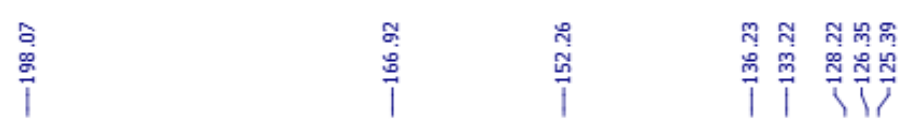

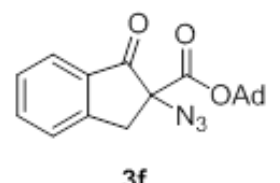

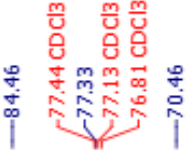

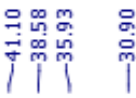

${ }^{13}$ C NMR

Solvent: $\mathbf{C D C l}_{\mathbf{3}}$

Frequency: $100.6001 \mathrm{MHz}$

$3 f$

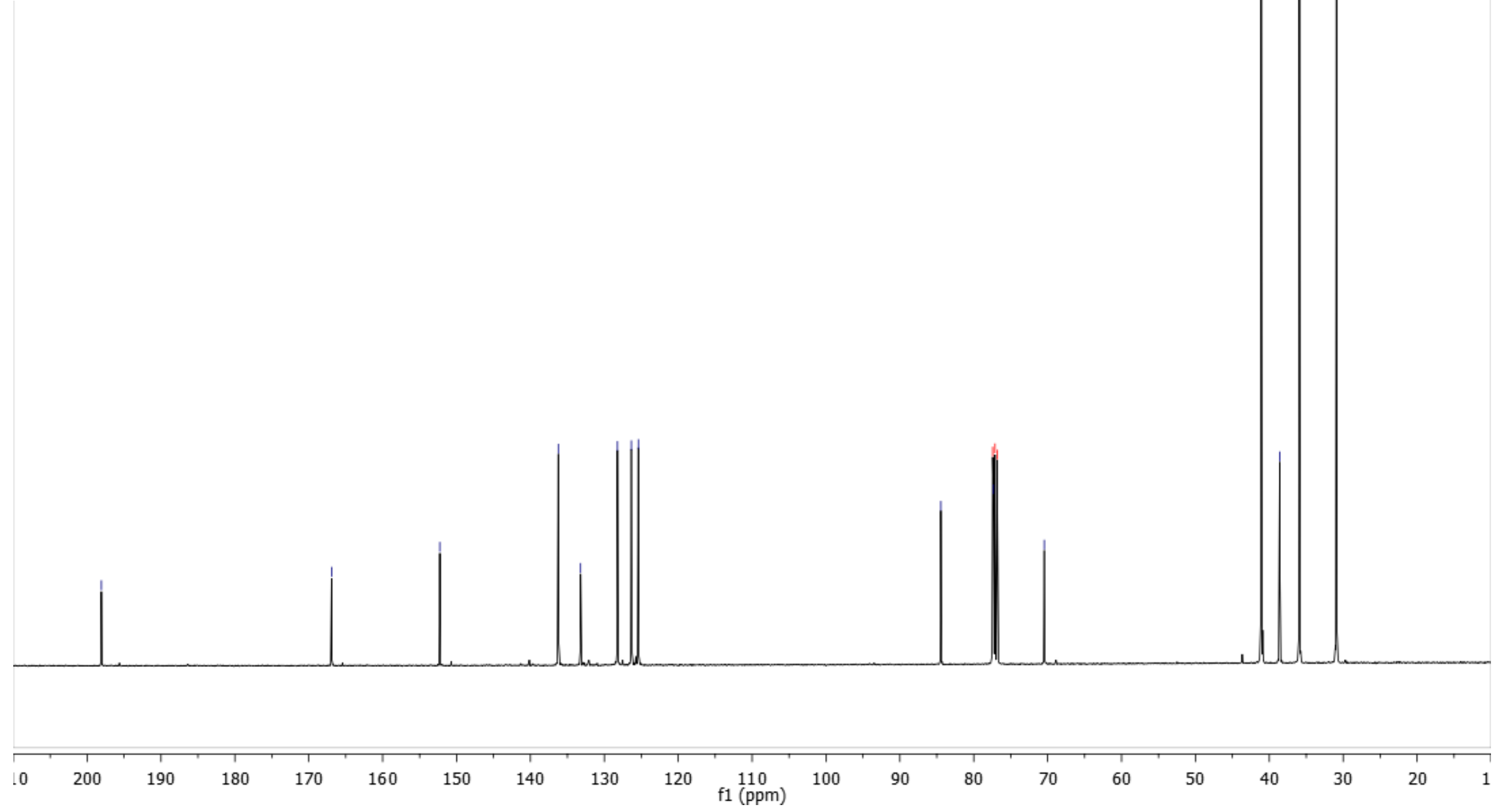




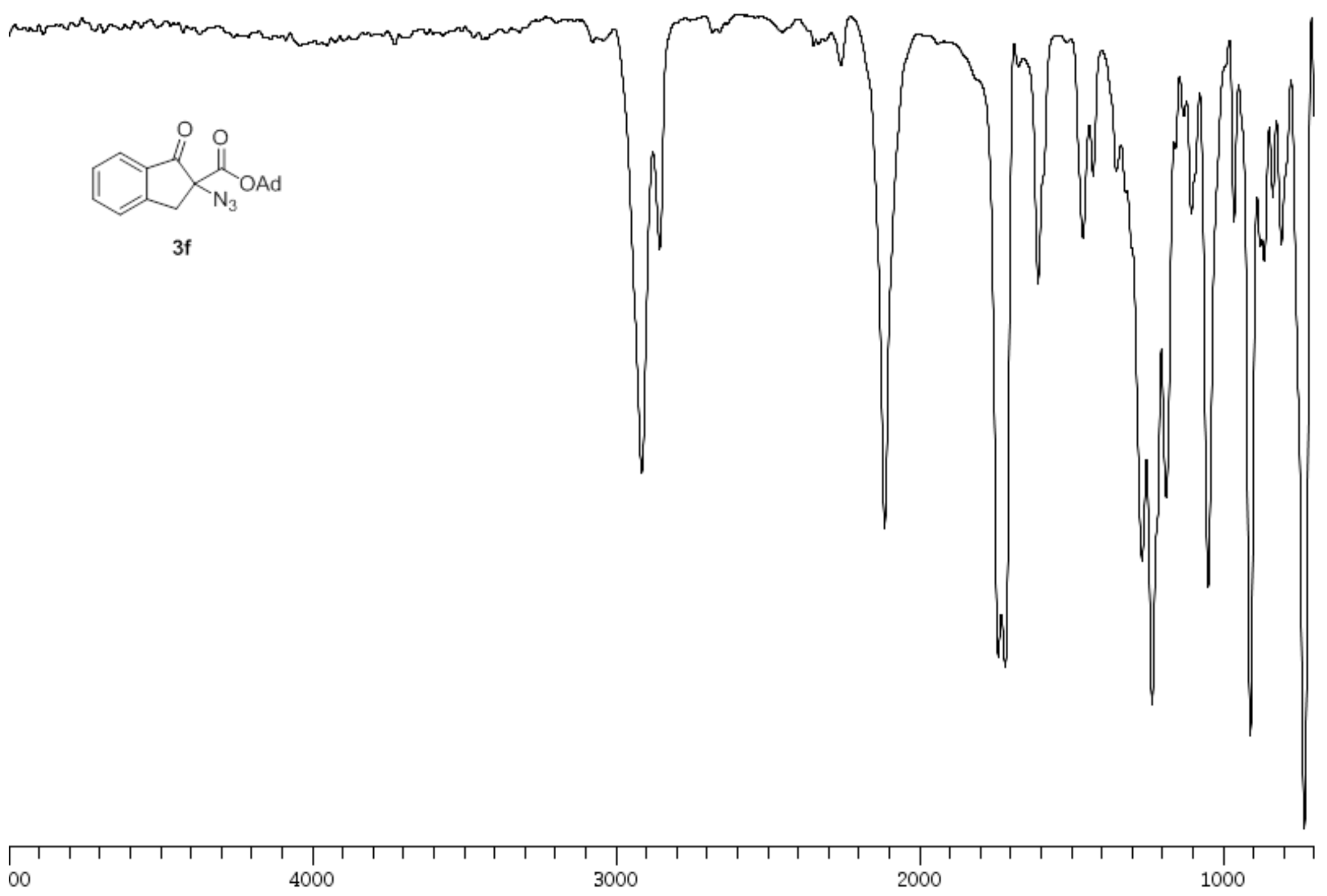




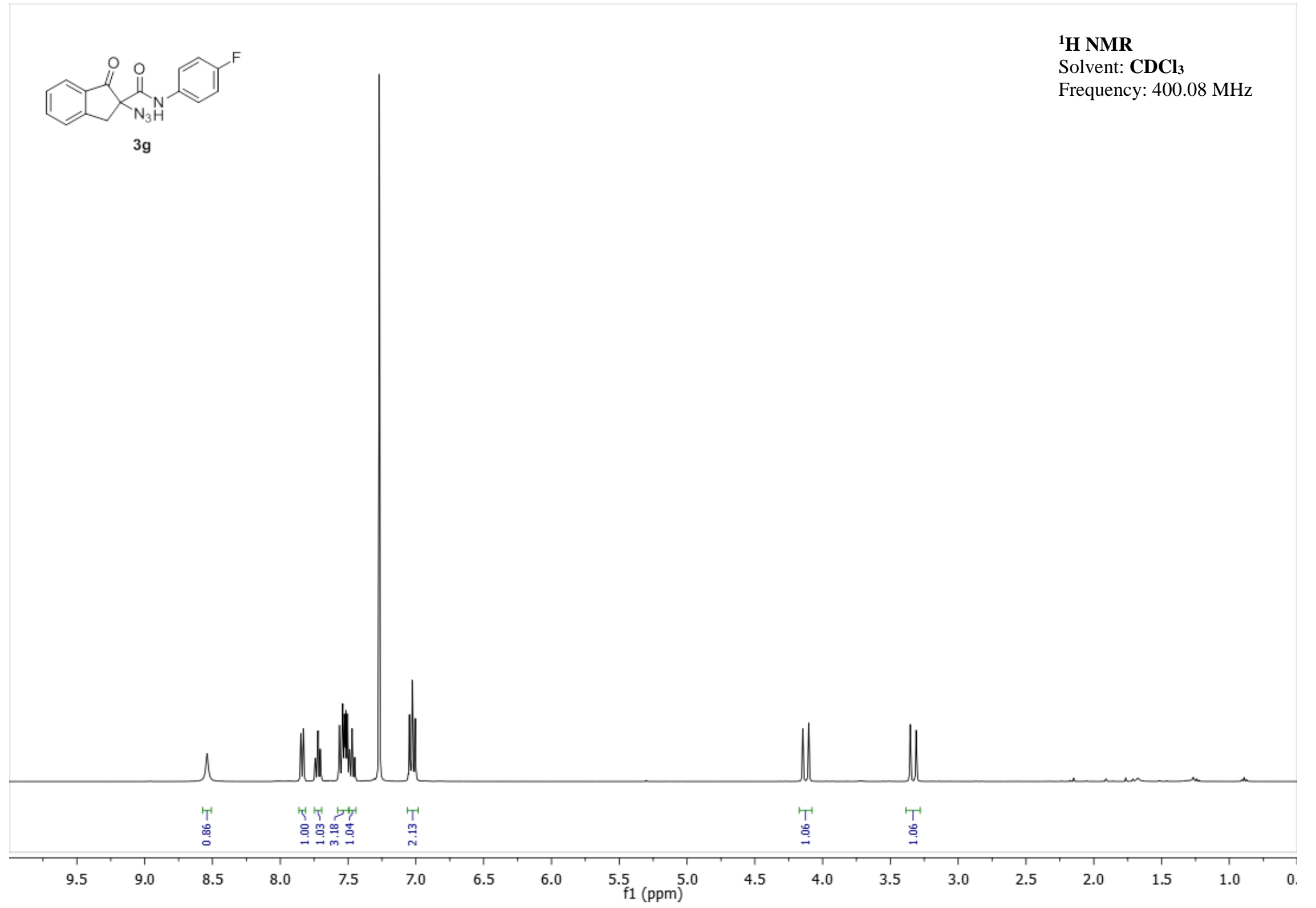



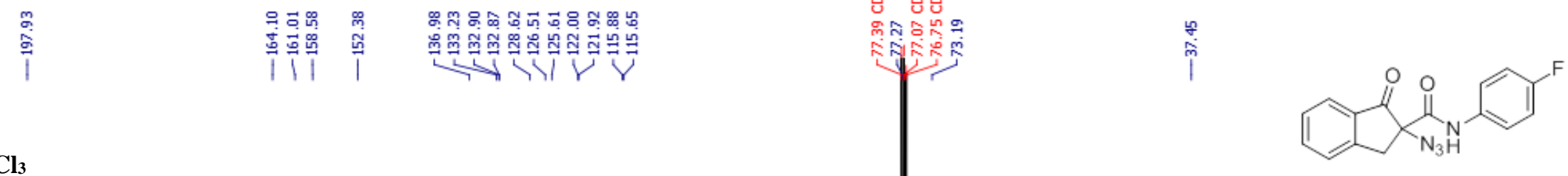

$3 \mathrm{~g}$

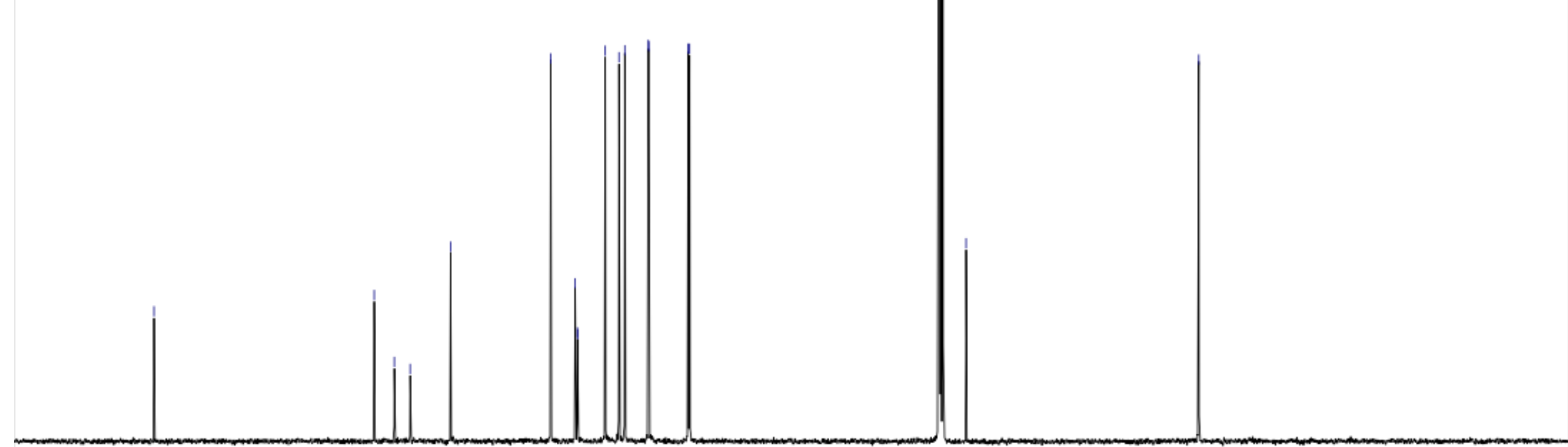

$210 \quad 200$ 


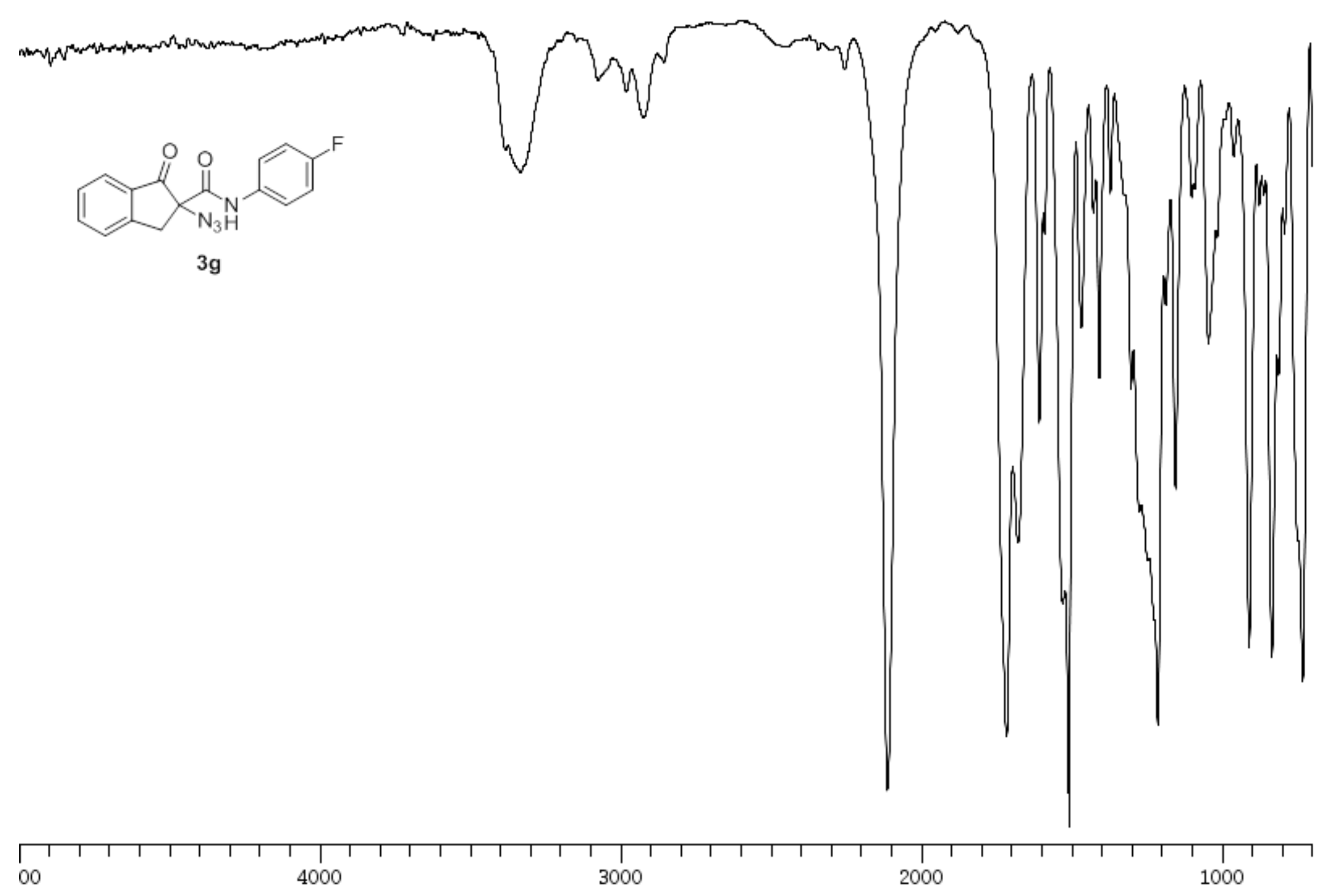




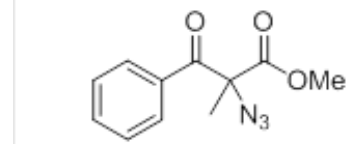

$3 \mathrm{~h}$
${ }^{1}$ H NMR

Solvent: $\mathbf{C D C l}_{3}$

Frequency: $400.08 \mathrm{MHz}$

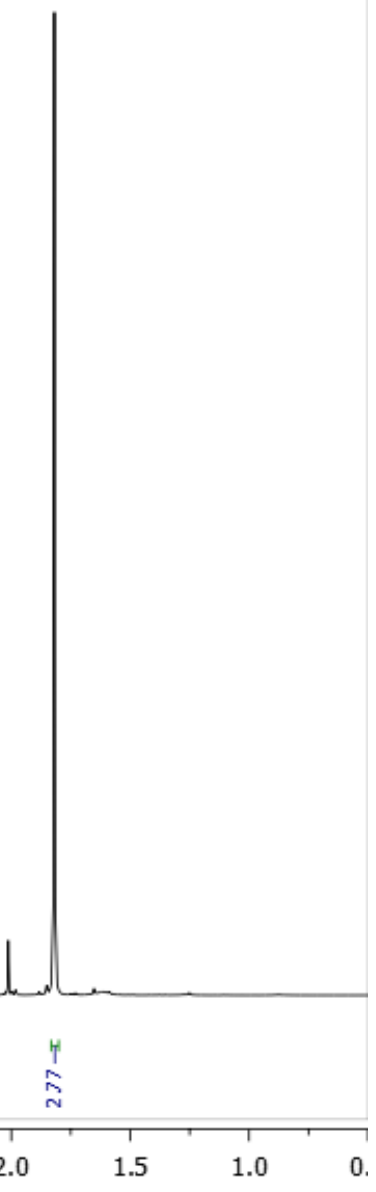




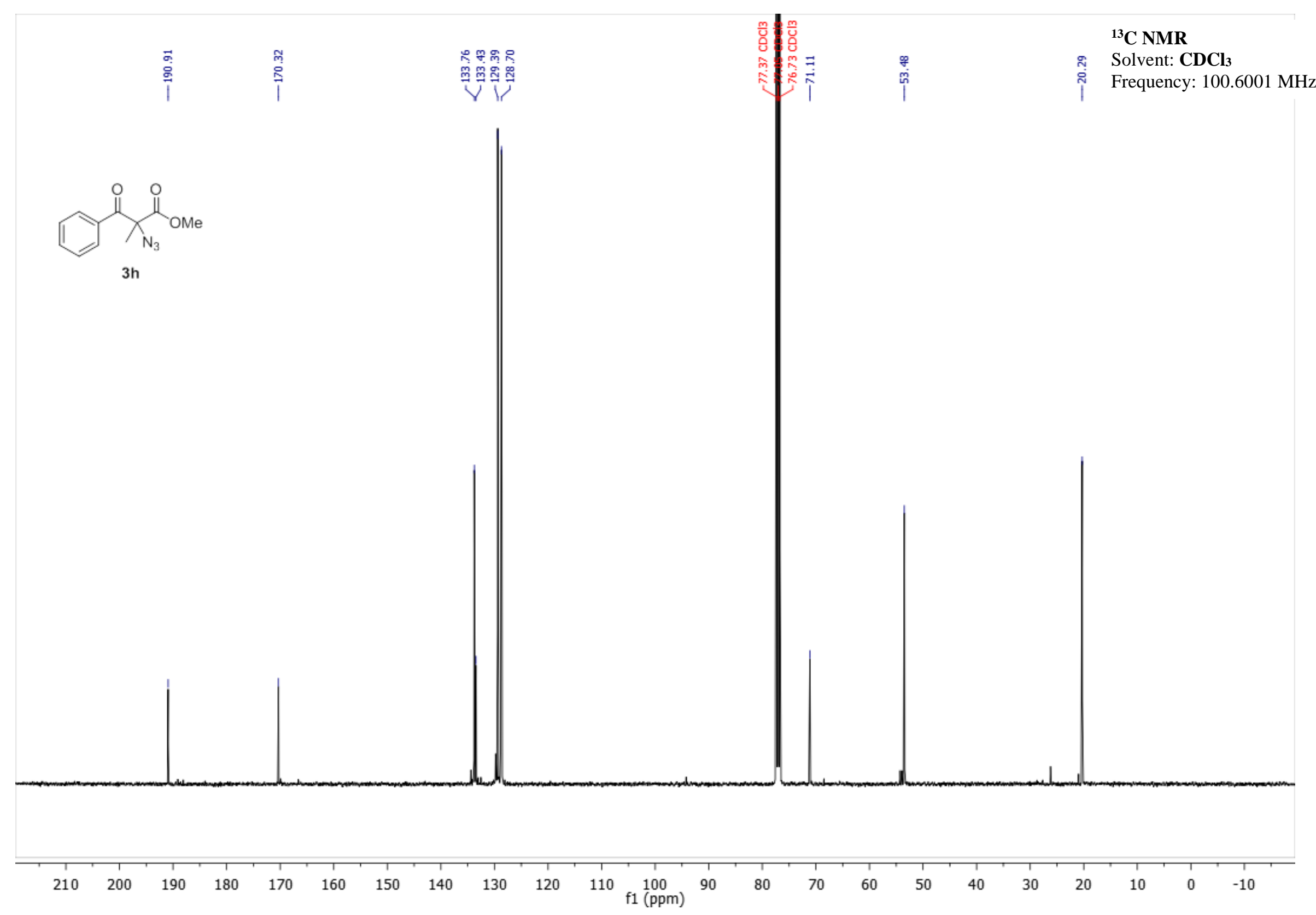




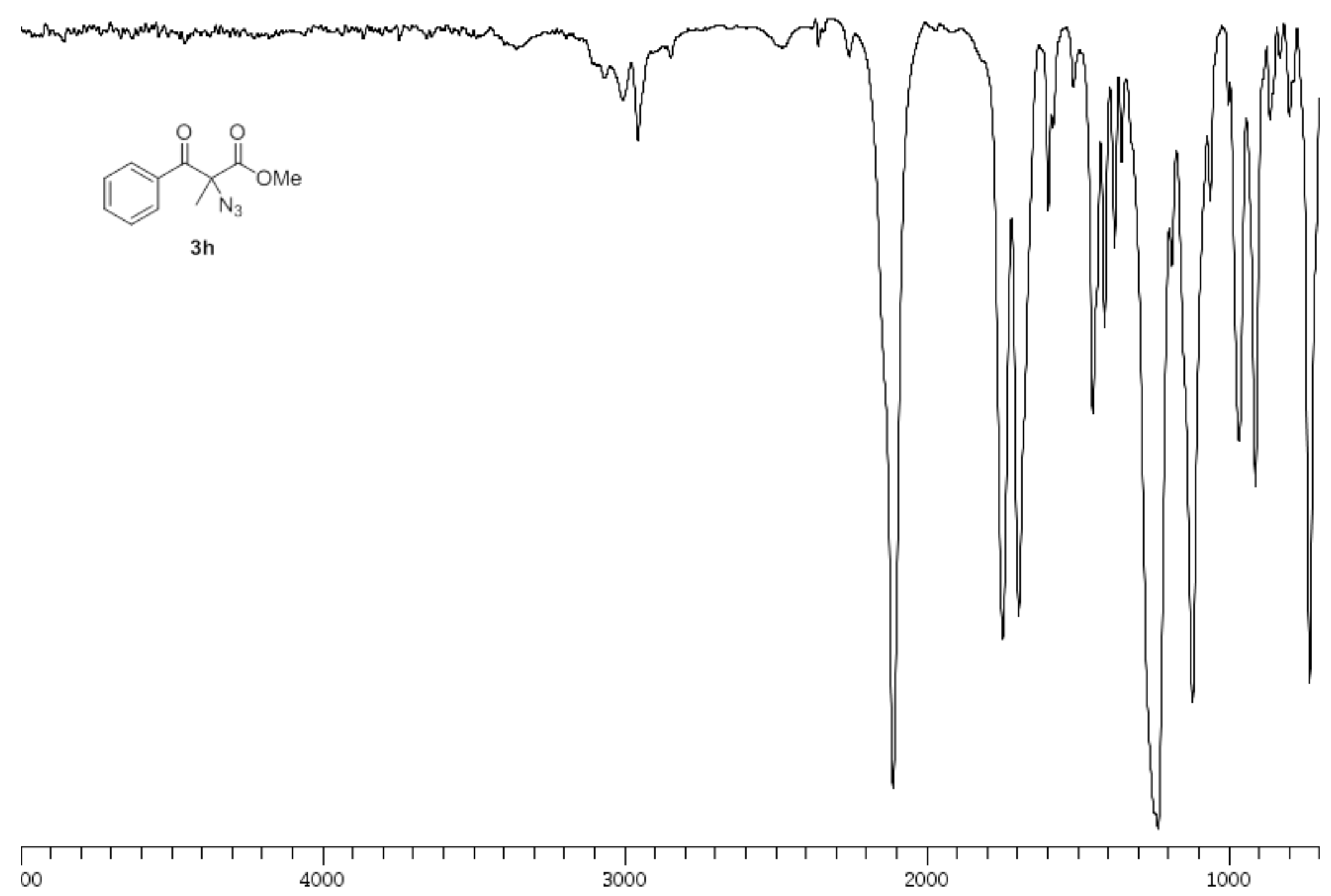




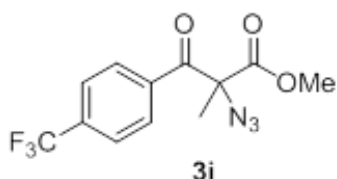

$3 i$
${ }^{1}$ H NMR

Solvent: $\mathbf{C D C l}_{3}$

Frequency: $400.08 \mathrm{MHz}$

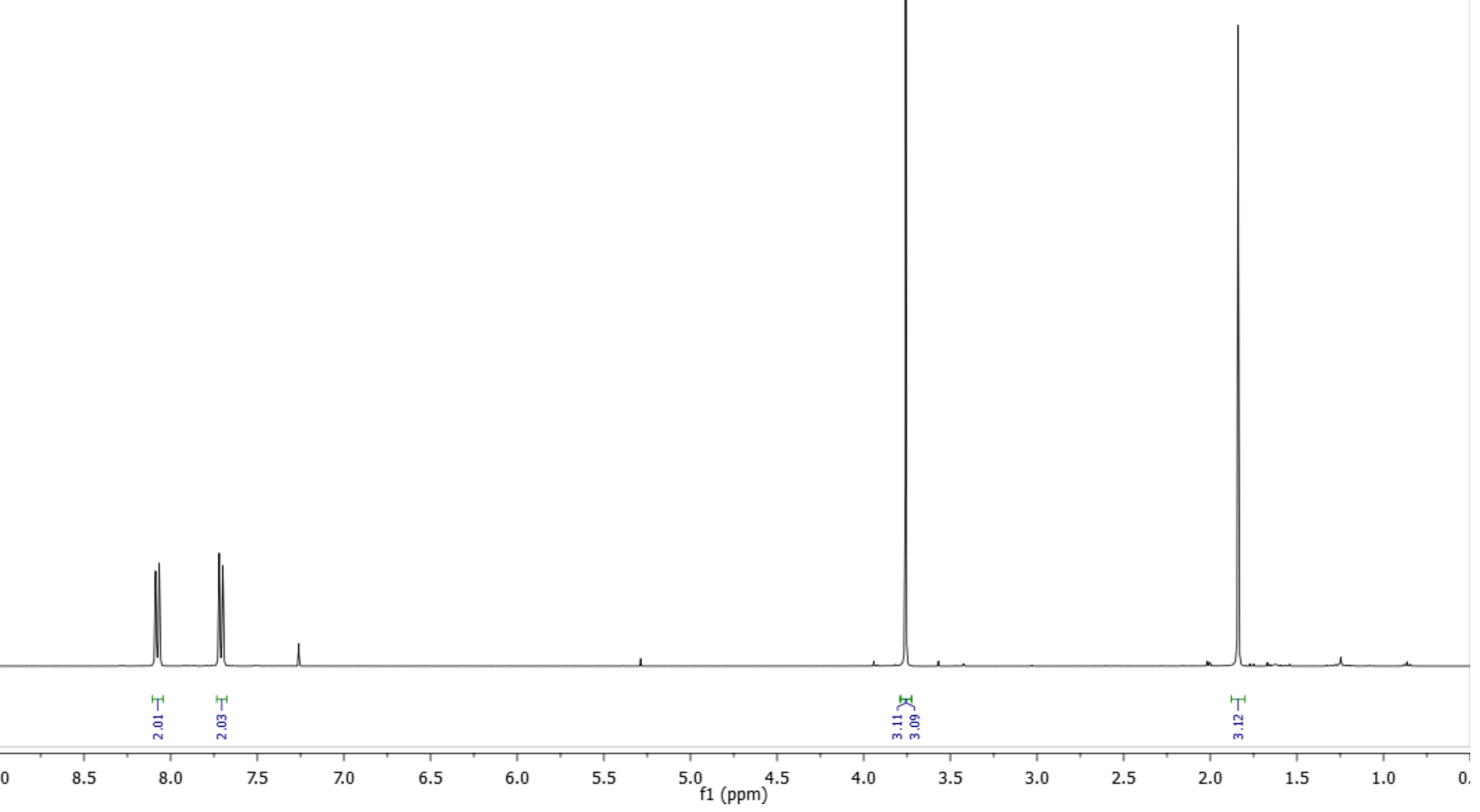



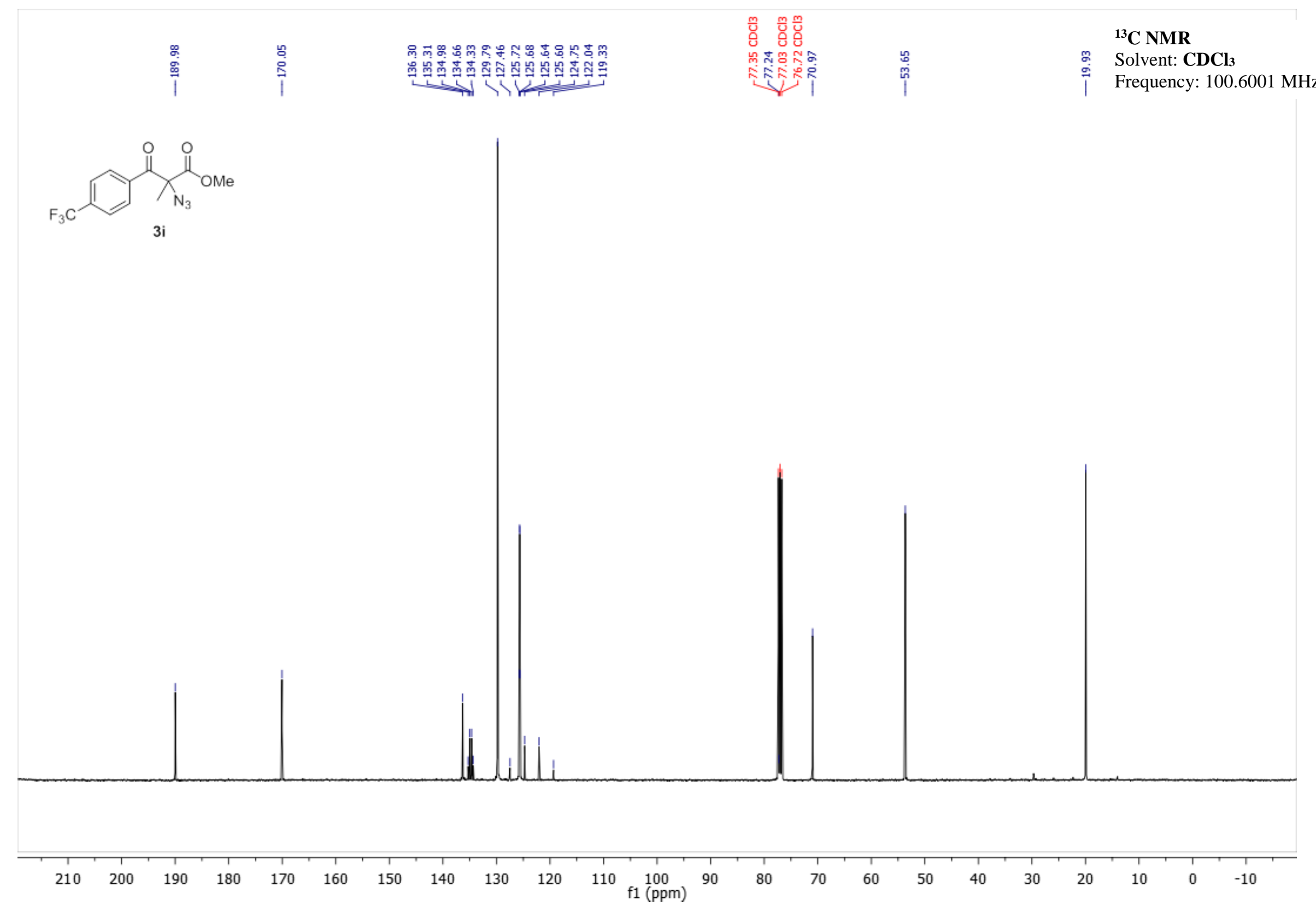


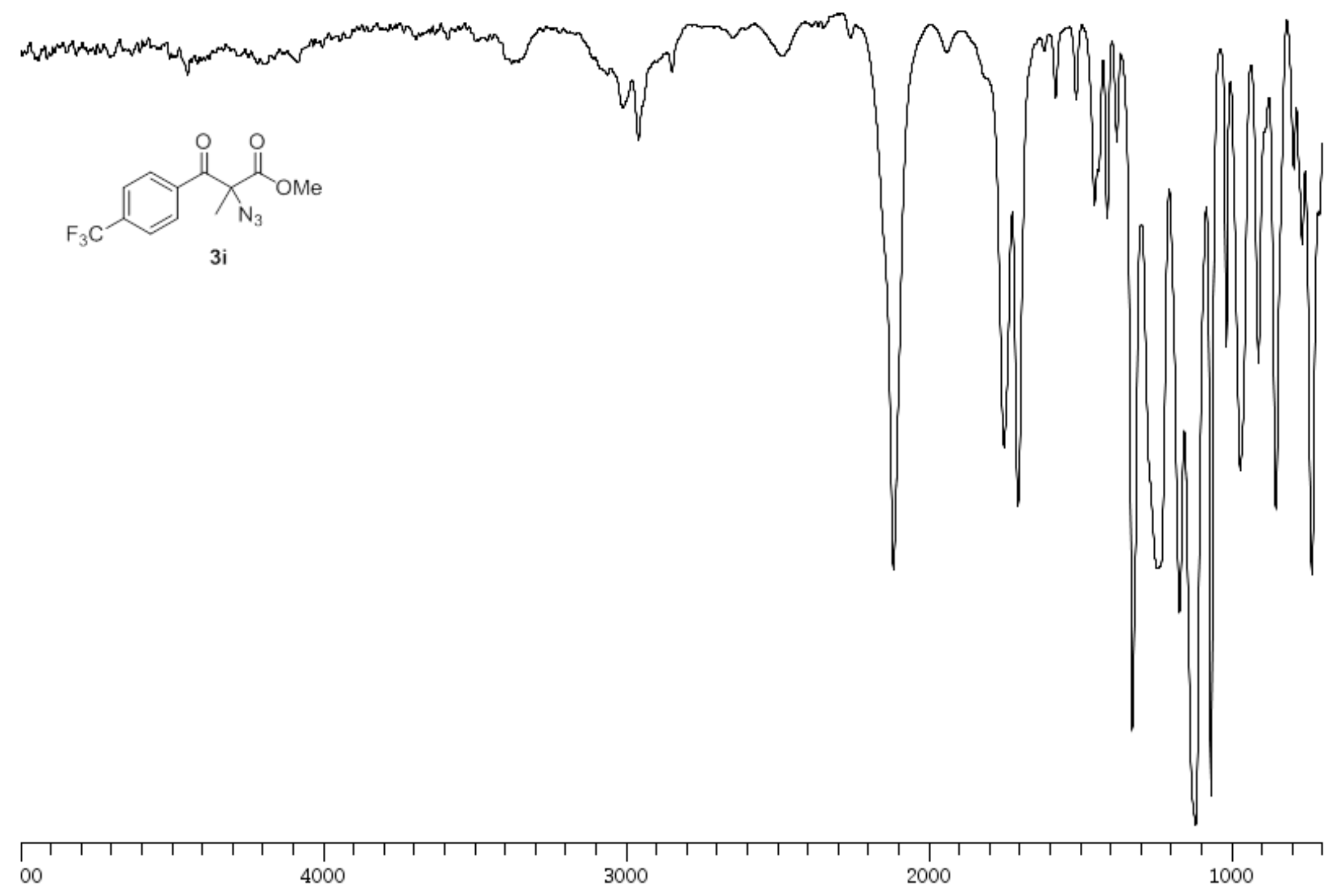




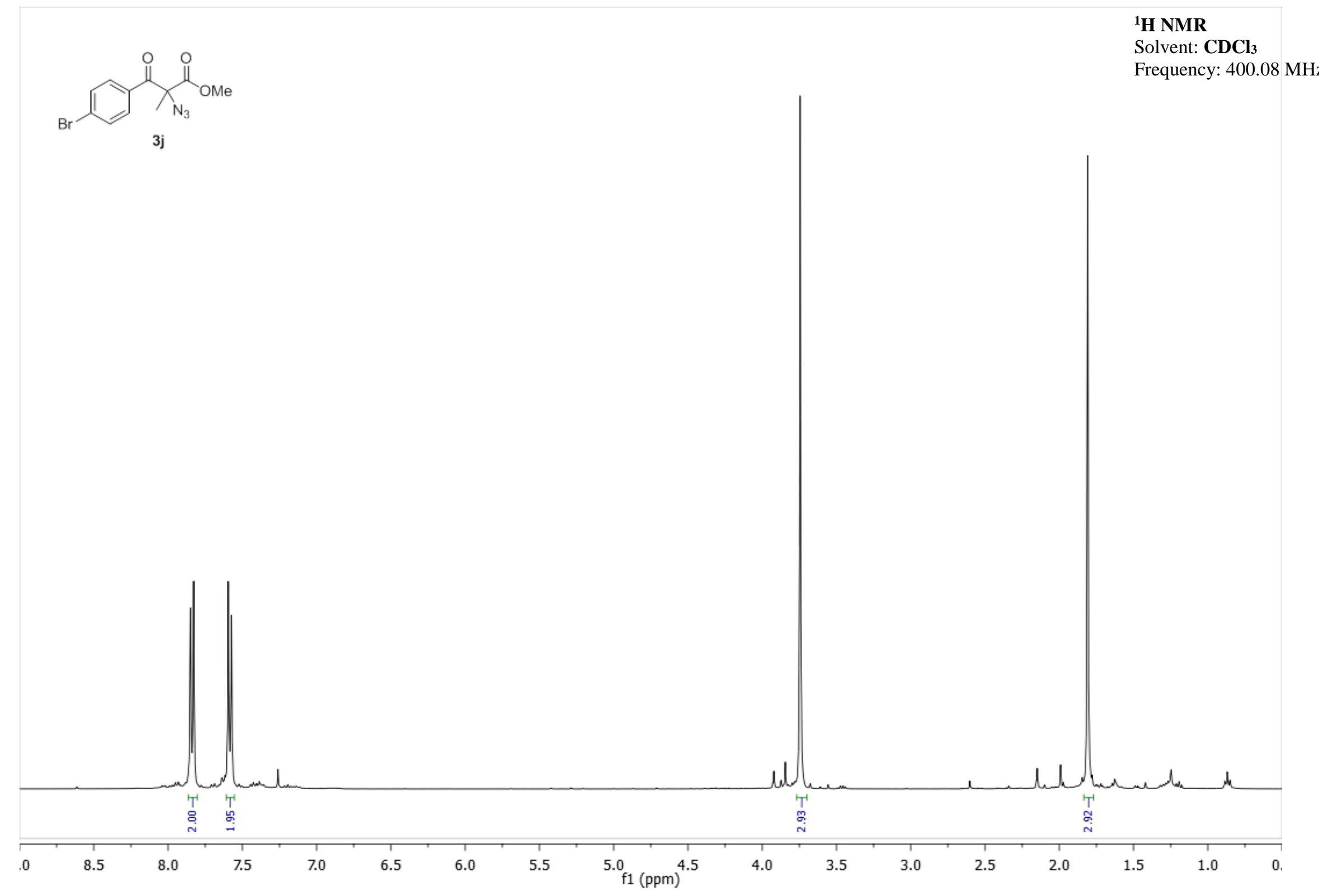



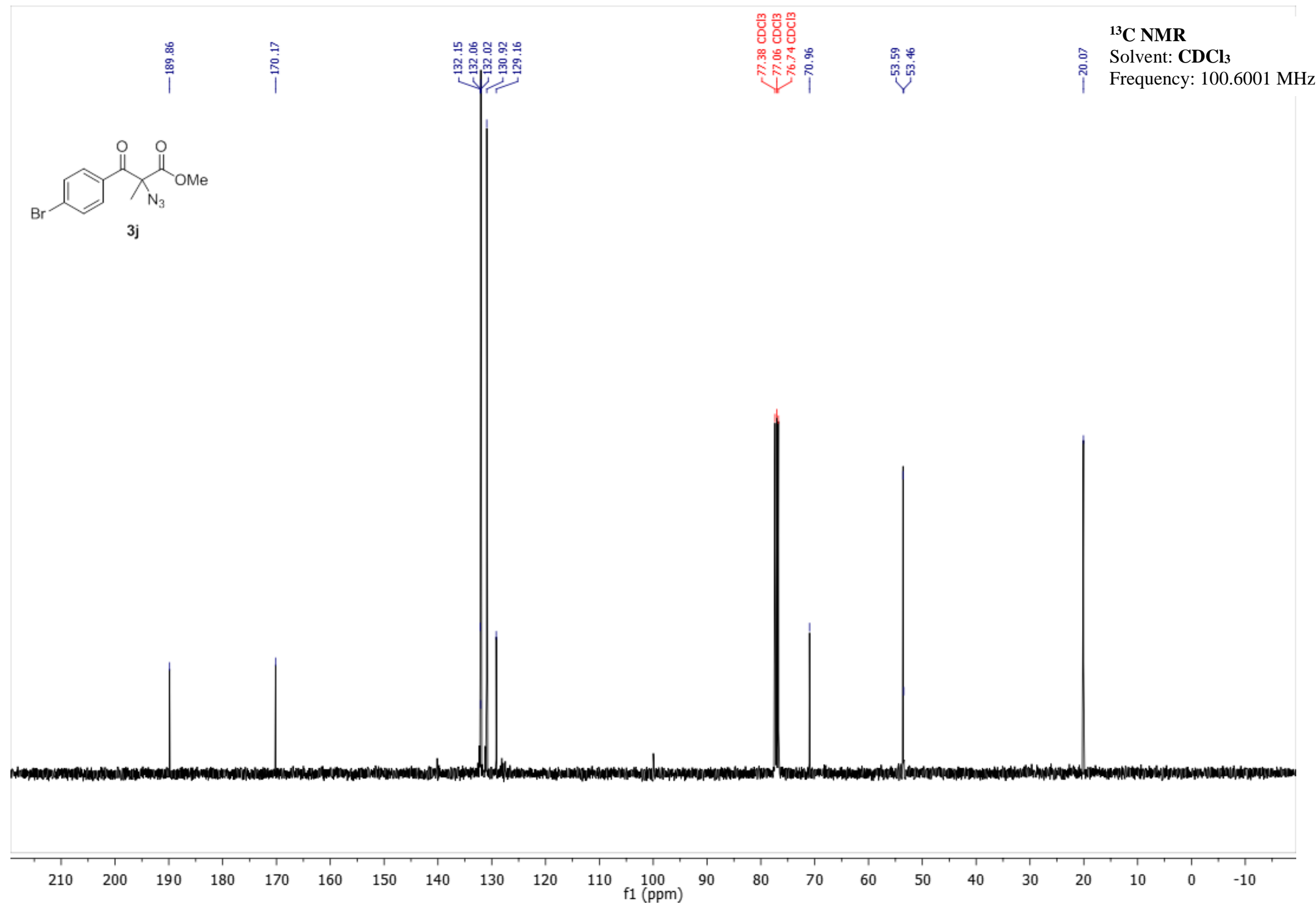


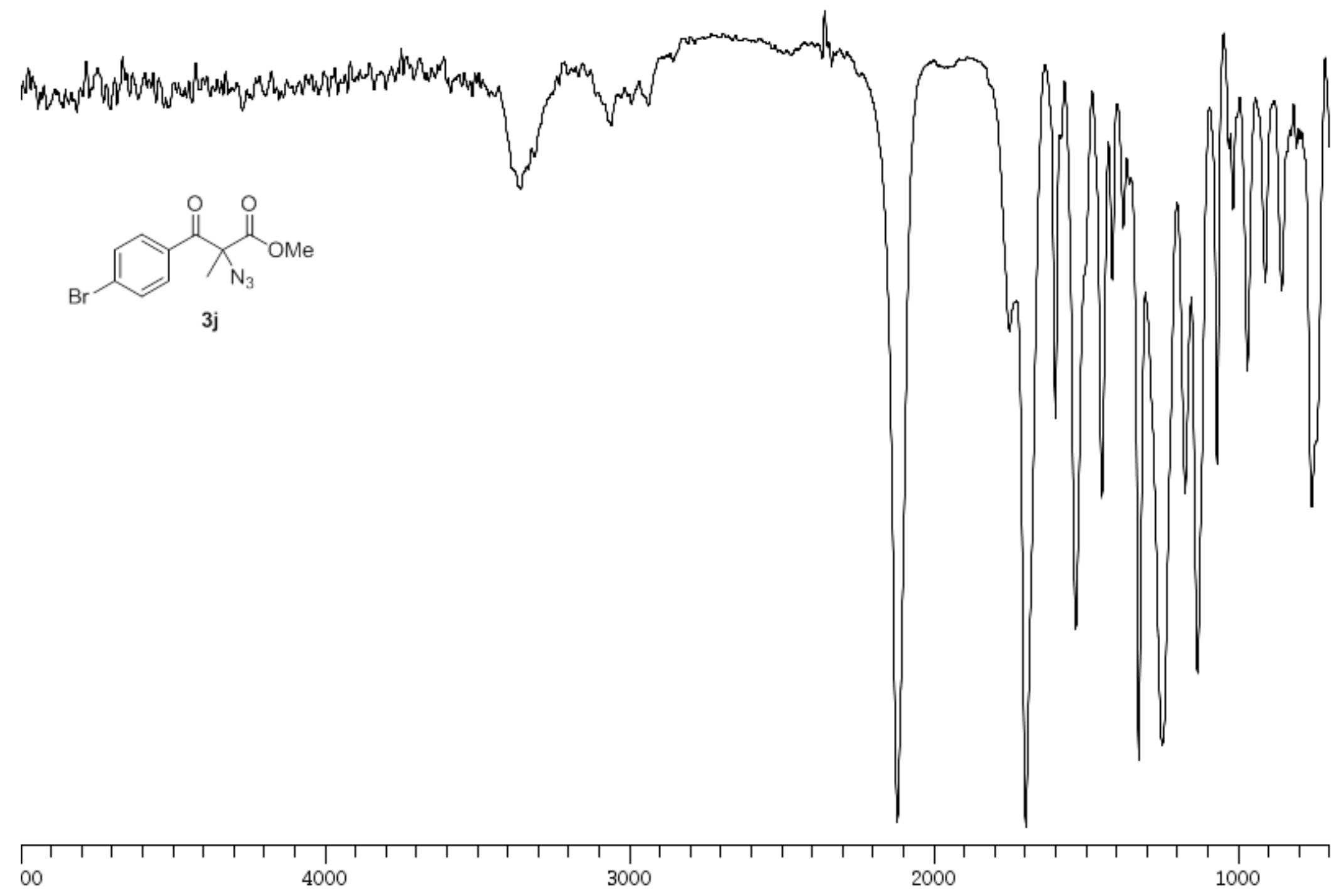




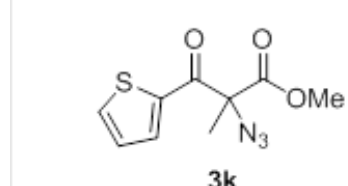

$3 \mathrm{k}$
${ }^{1}$ H NMR

Solvent: $\mathbf{C D C l}_{\mathbf{3}}$

Frequency: $400.08 \mathrm{MHz}$

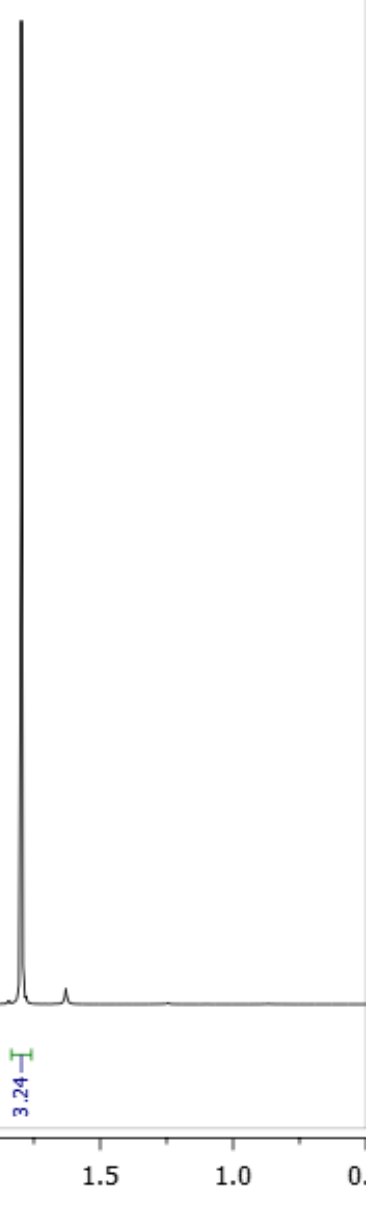




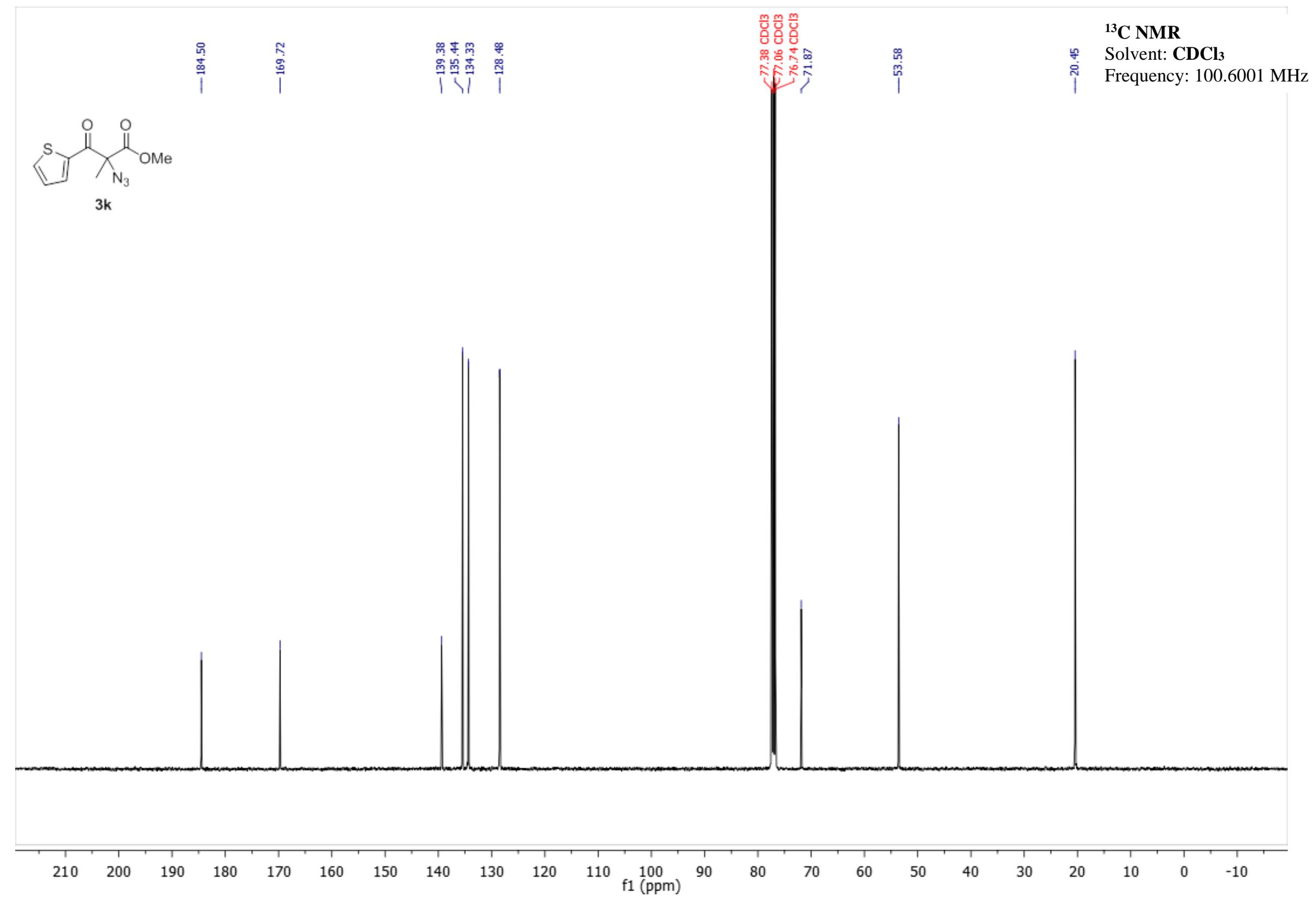




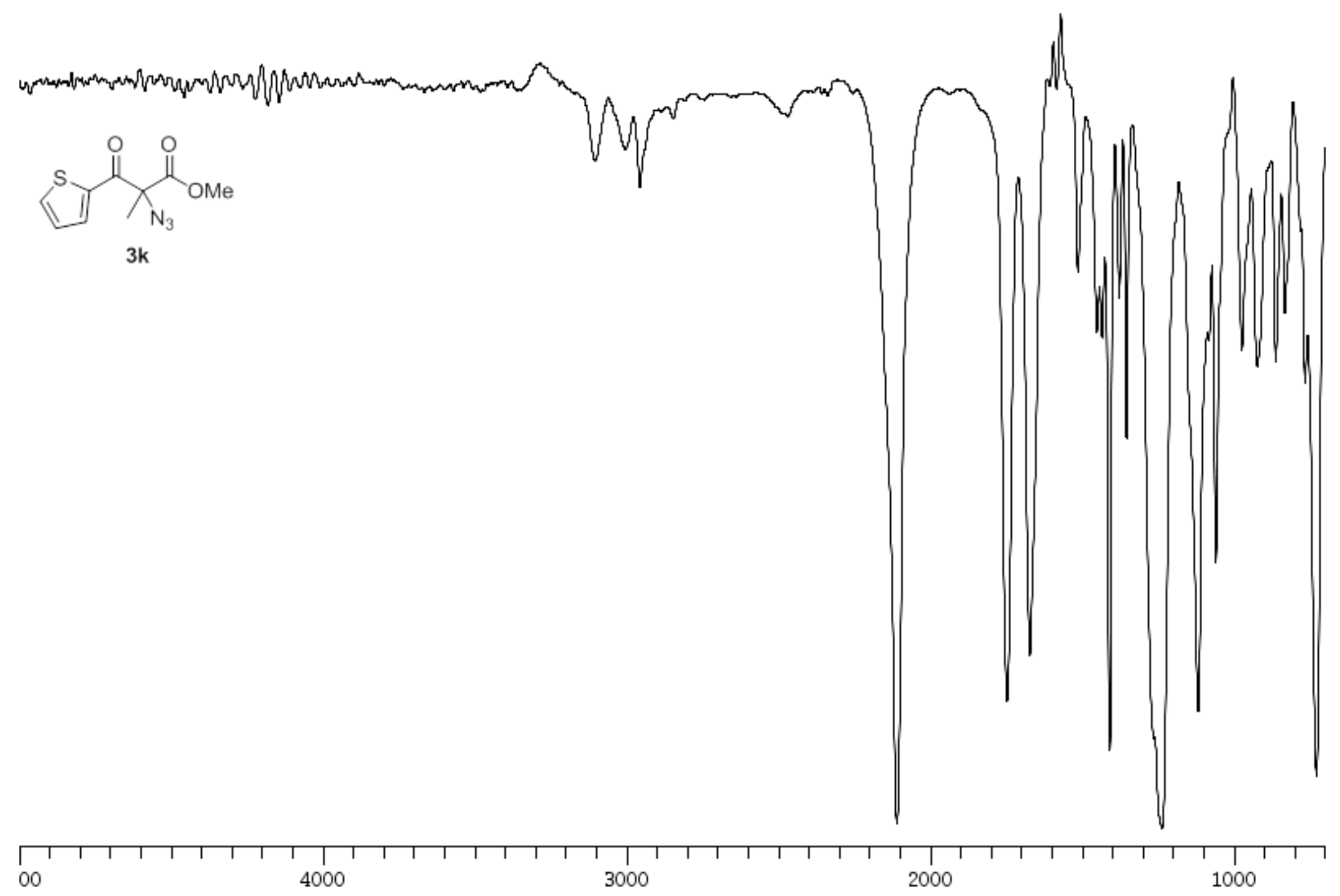




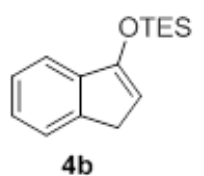

${ }^{1}$ H NMR

Solvent: $\mathbf{C D C l}_{\mathbf{3}}$

Frequency: $400.08 \mathrm{MHz}$

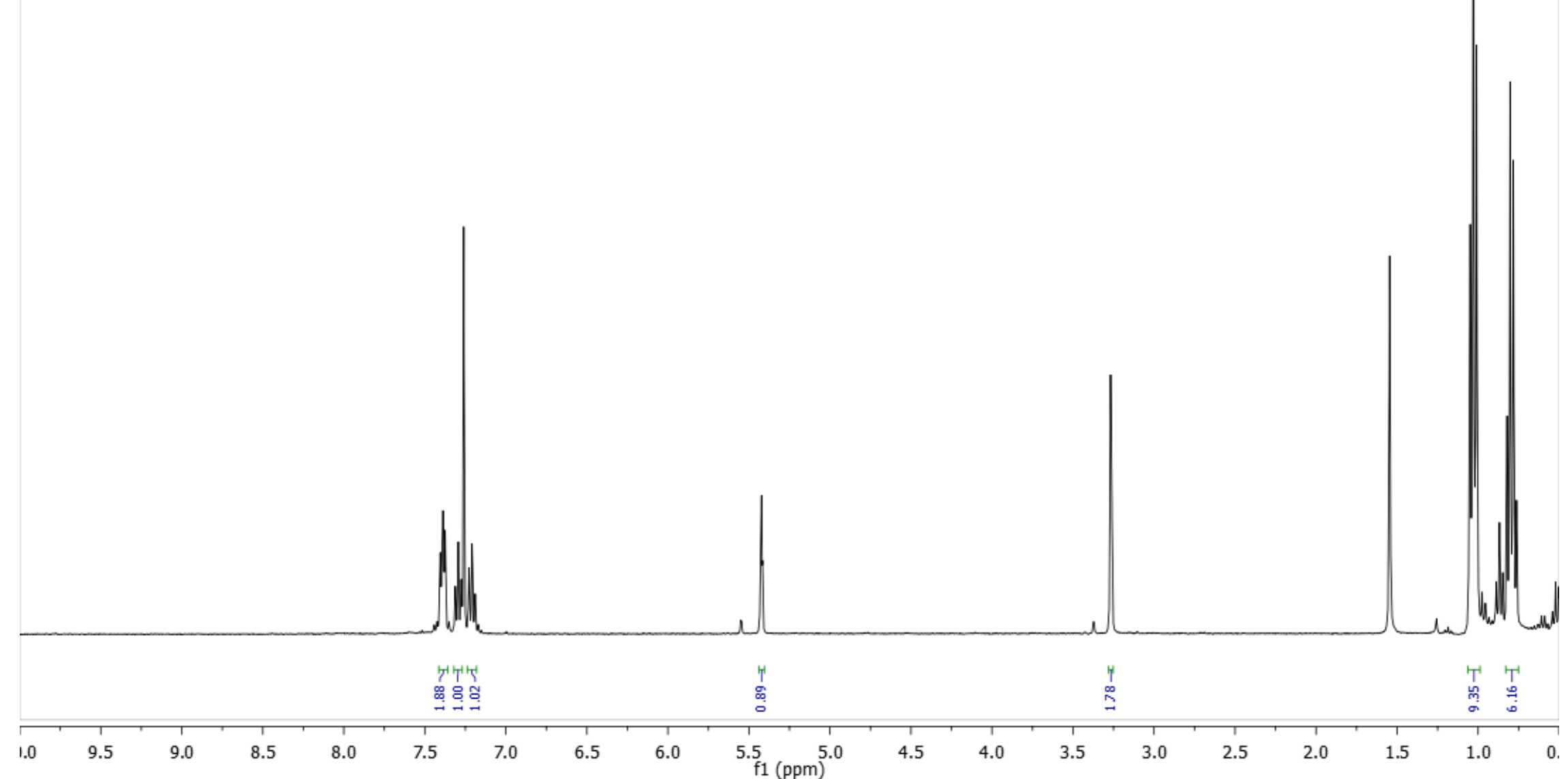




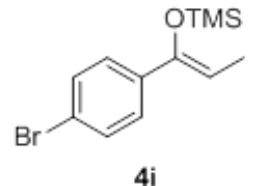

${ }^{1}$ H NMR

Solvent: $\mathbf{C D C l}_{\mathbf{3}}$

Frequency: $400.08 \mathrm{MHz}$

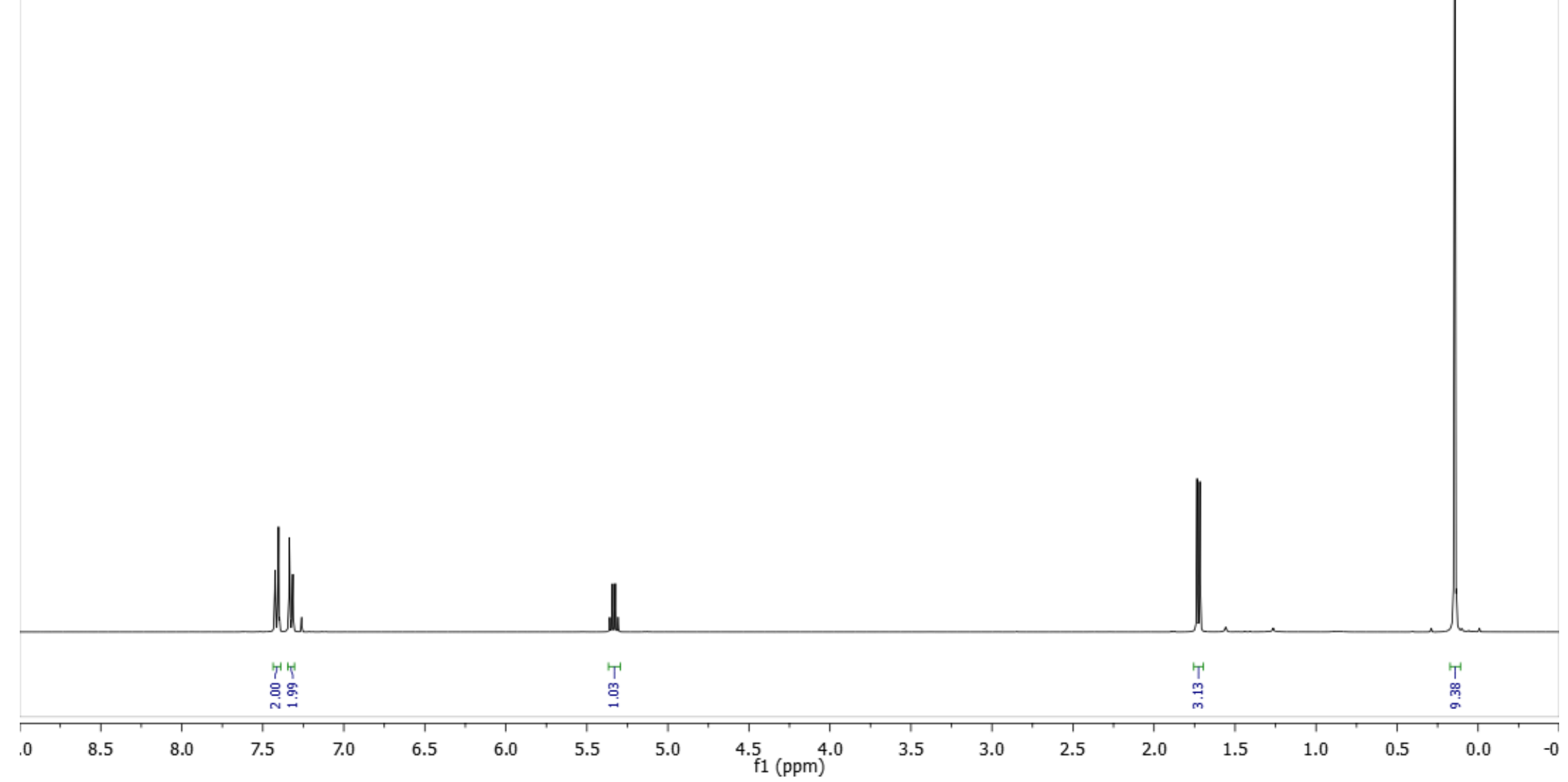




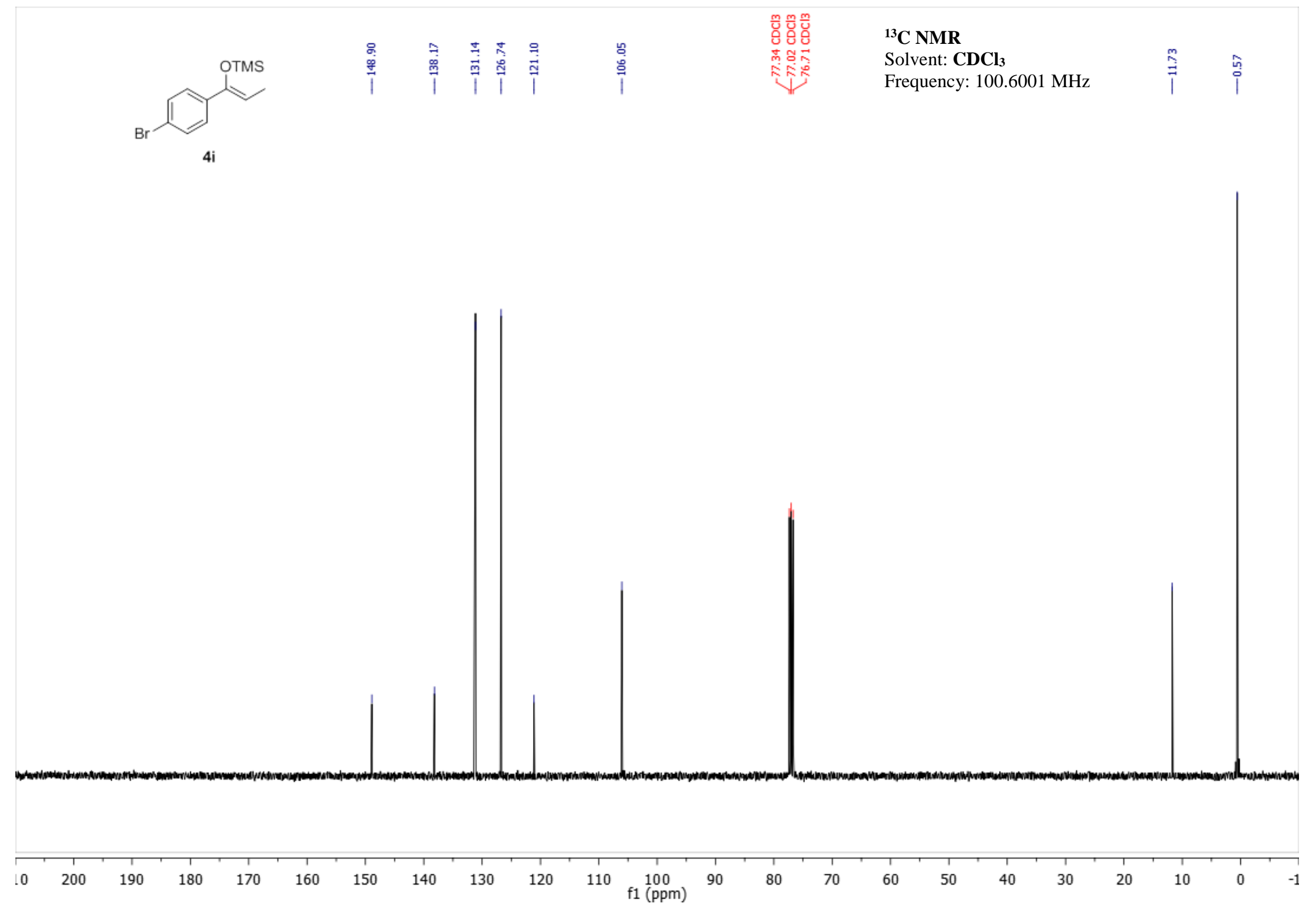




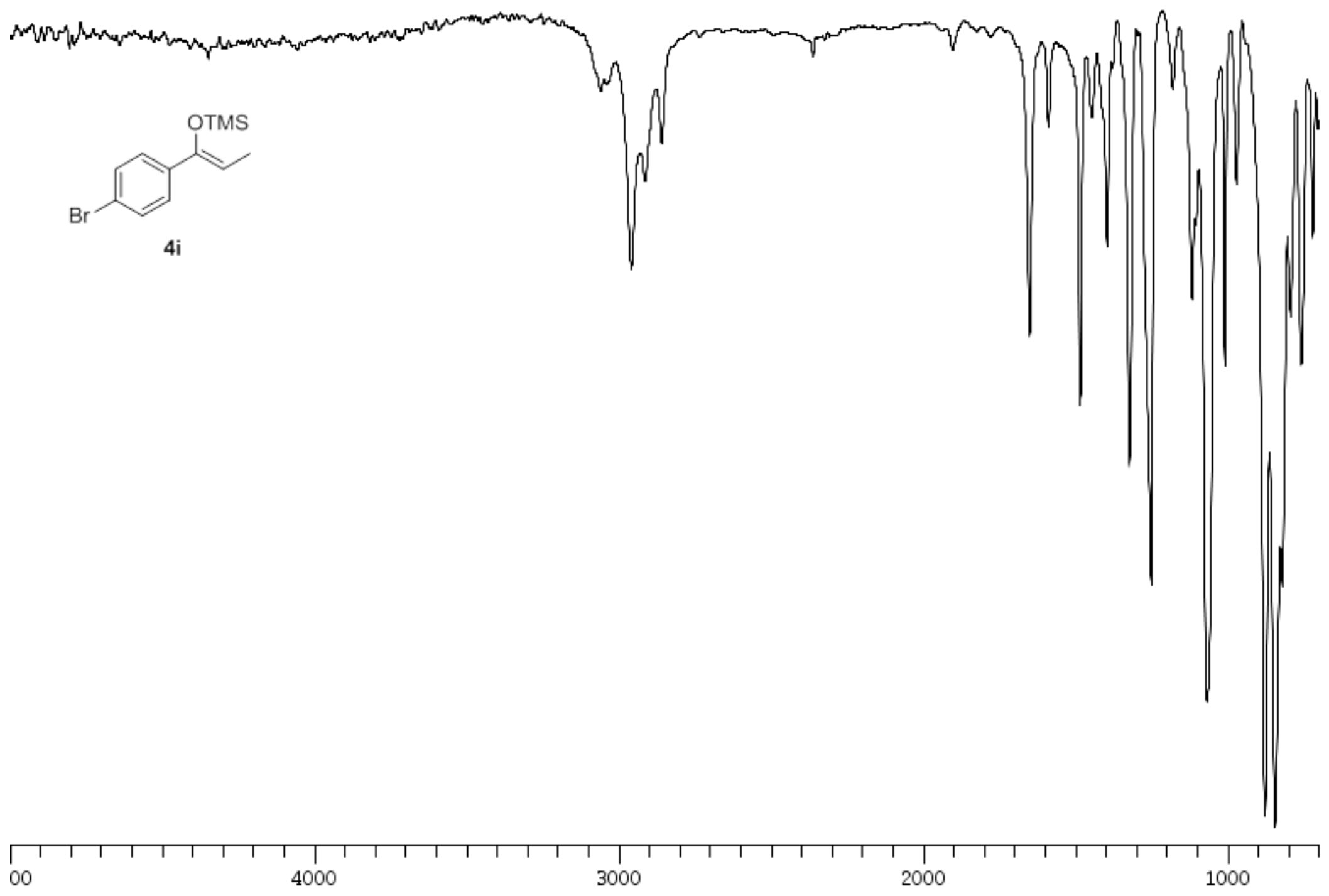




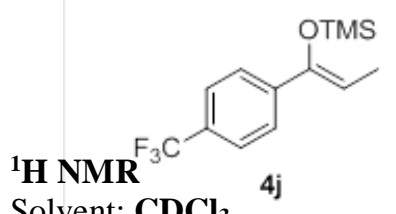

Solvent: $\mathbf{C D C l}_{3}$

Frequency: $400.08 \mathrm{MHz}$

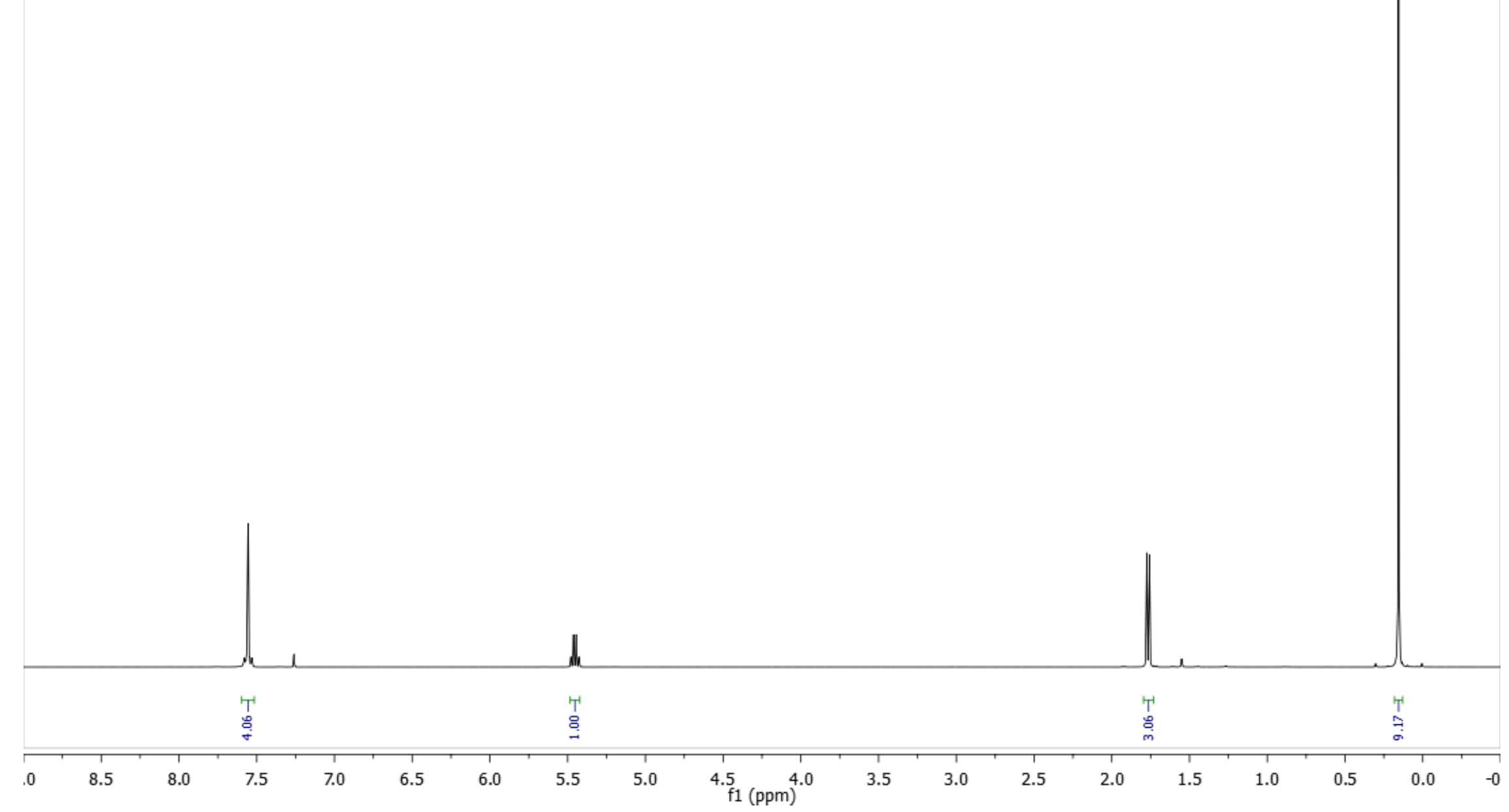



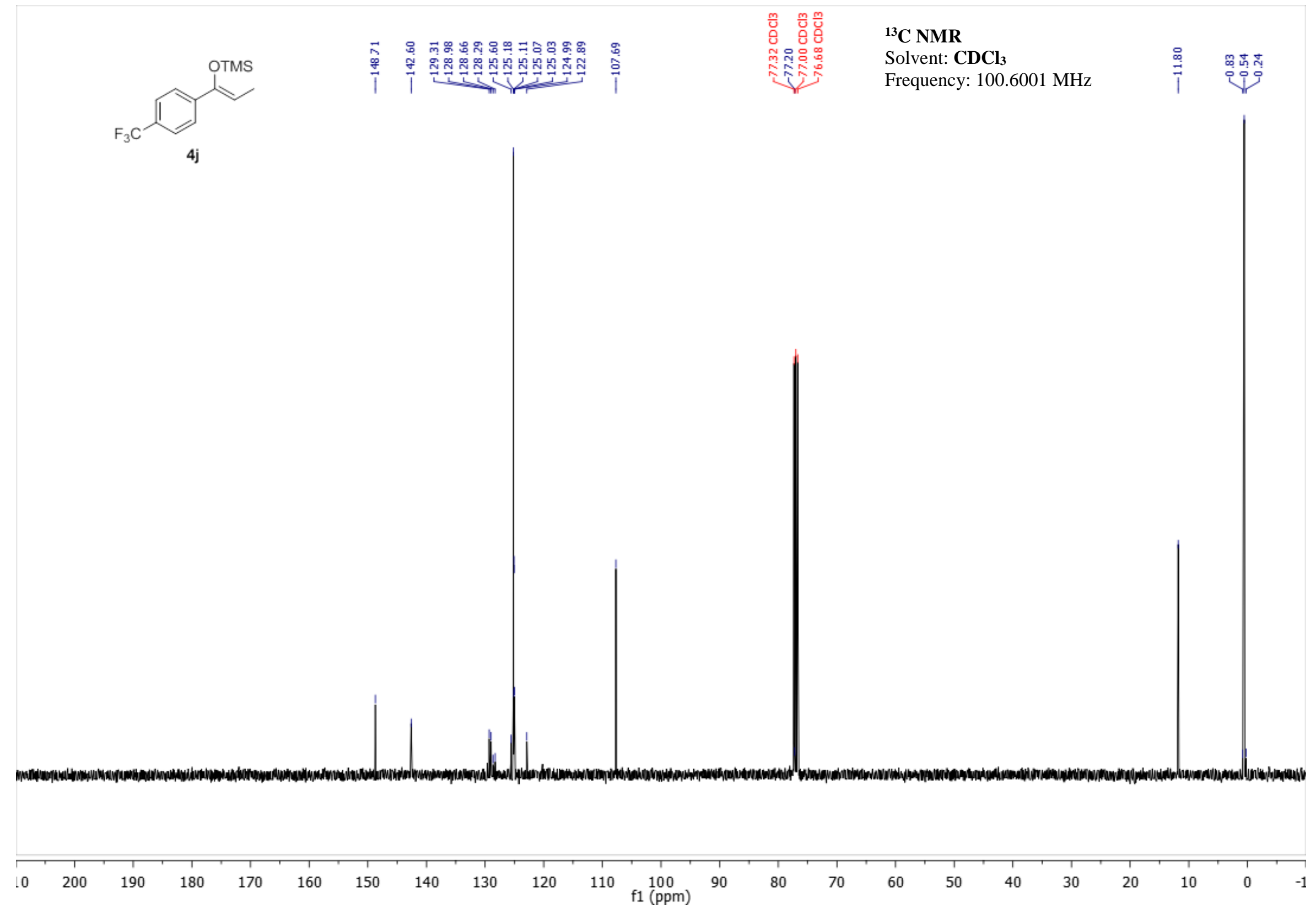


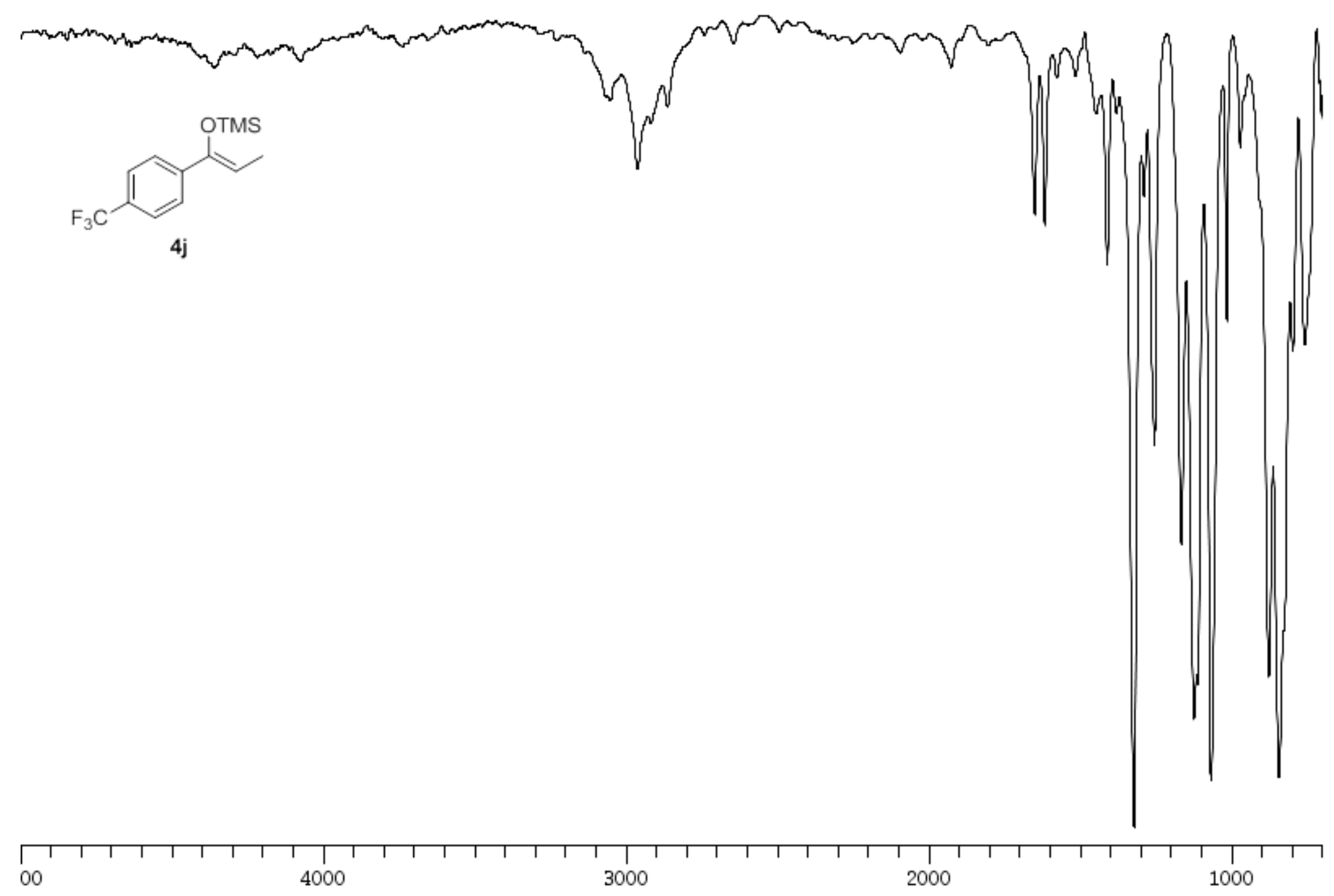




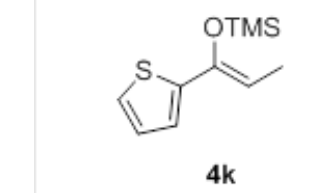

\section{${ }^{1}$ H NMR}

Solvent: $\mathbf{C D C l}_{3}$

Frequency: $400.08 \mathrm{MHz}$

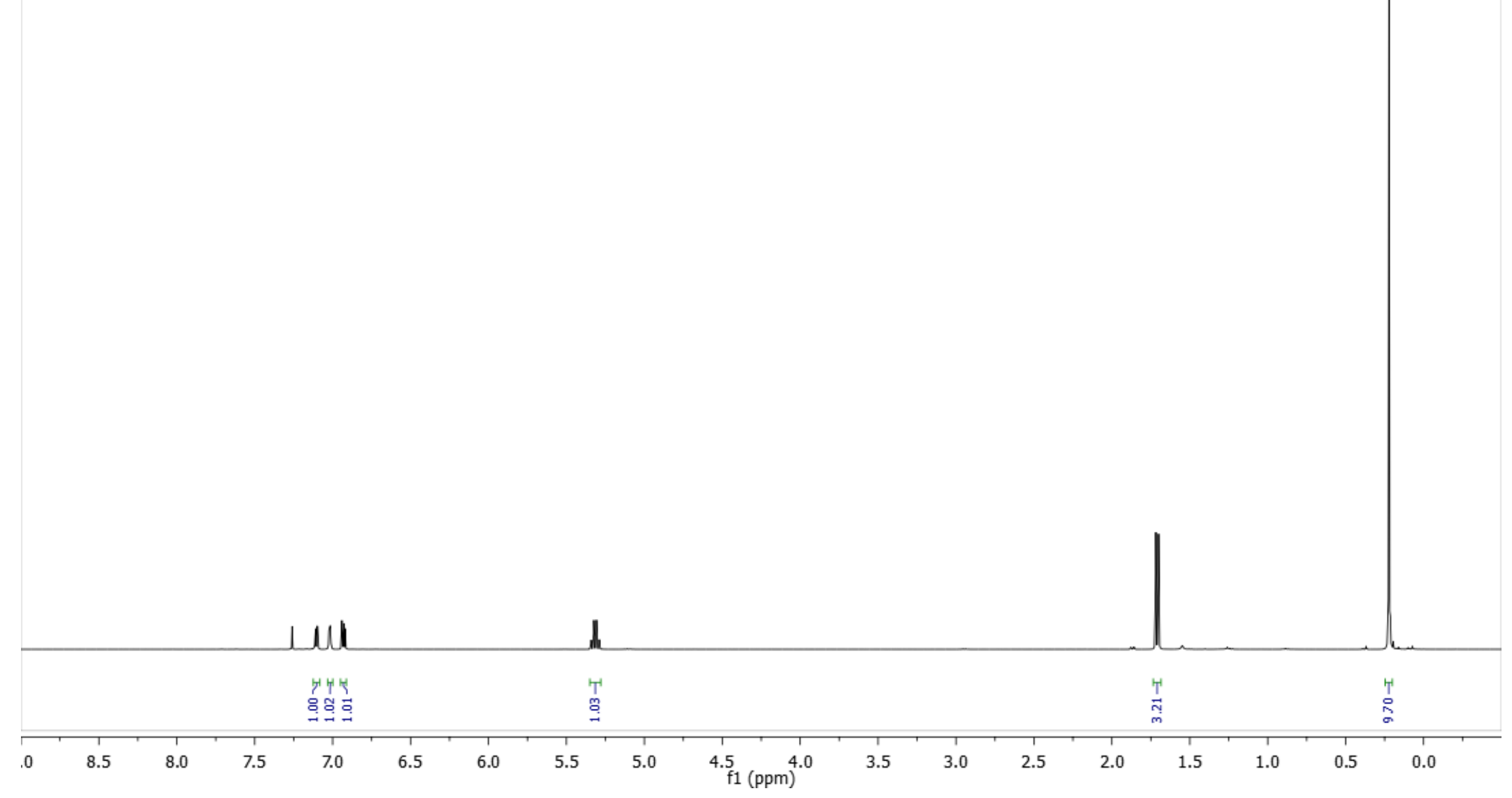




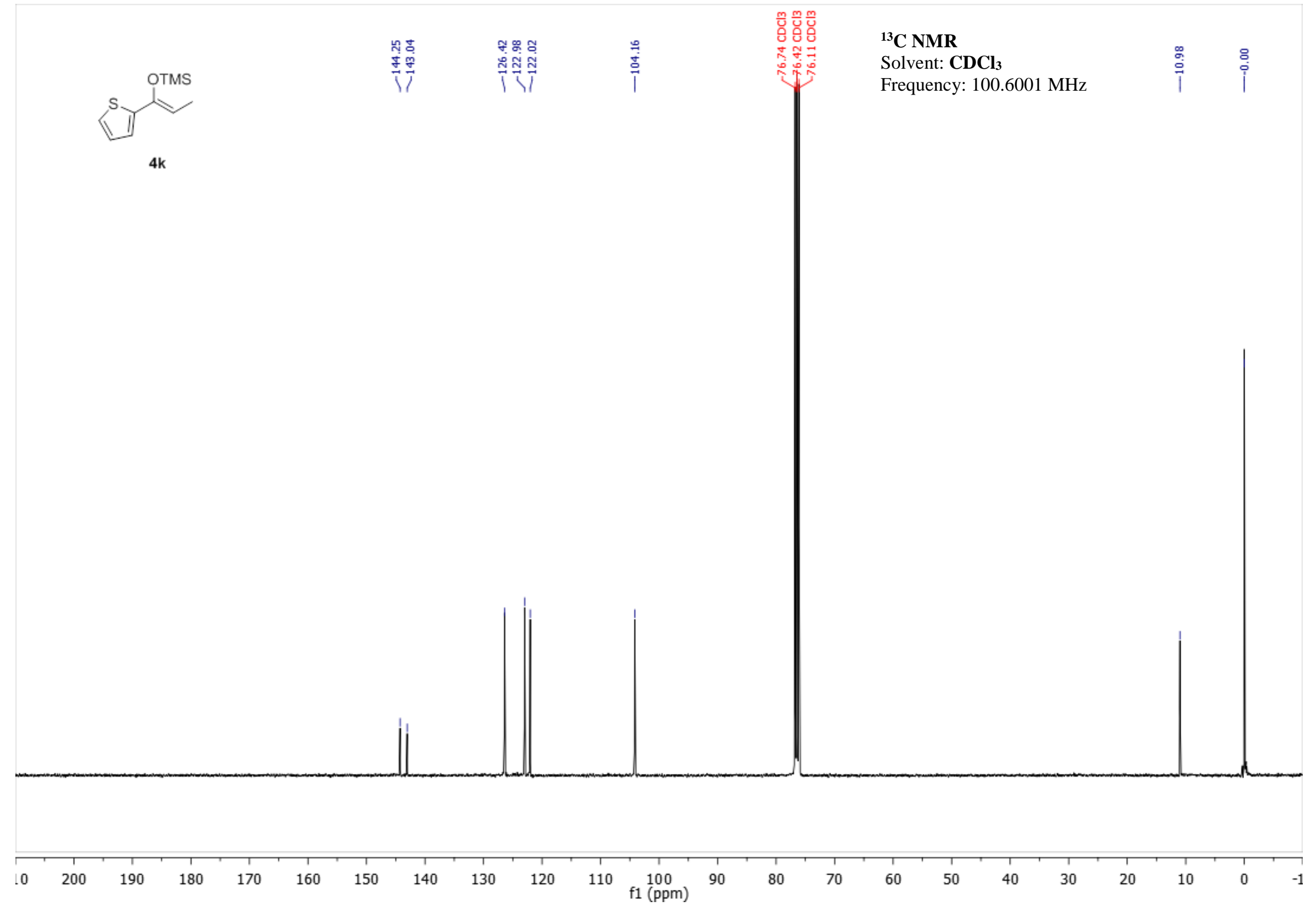




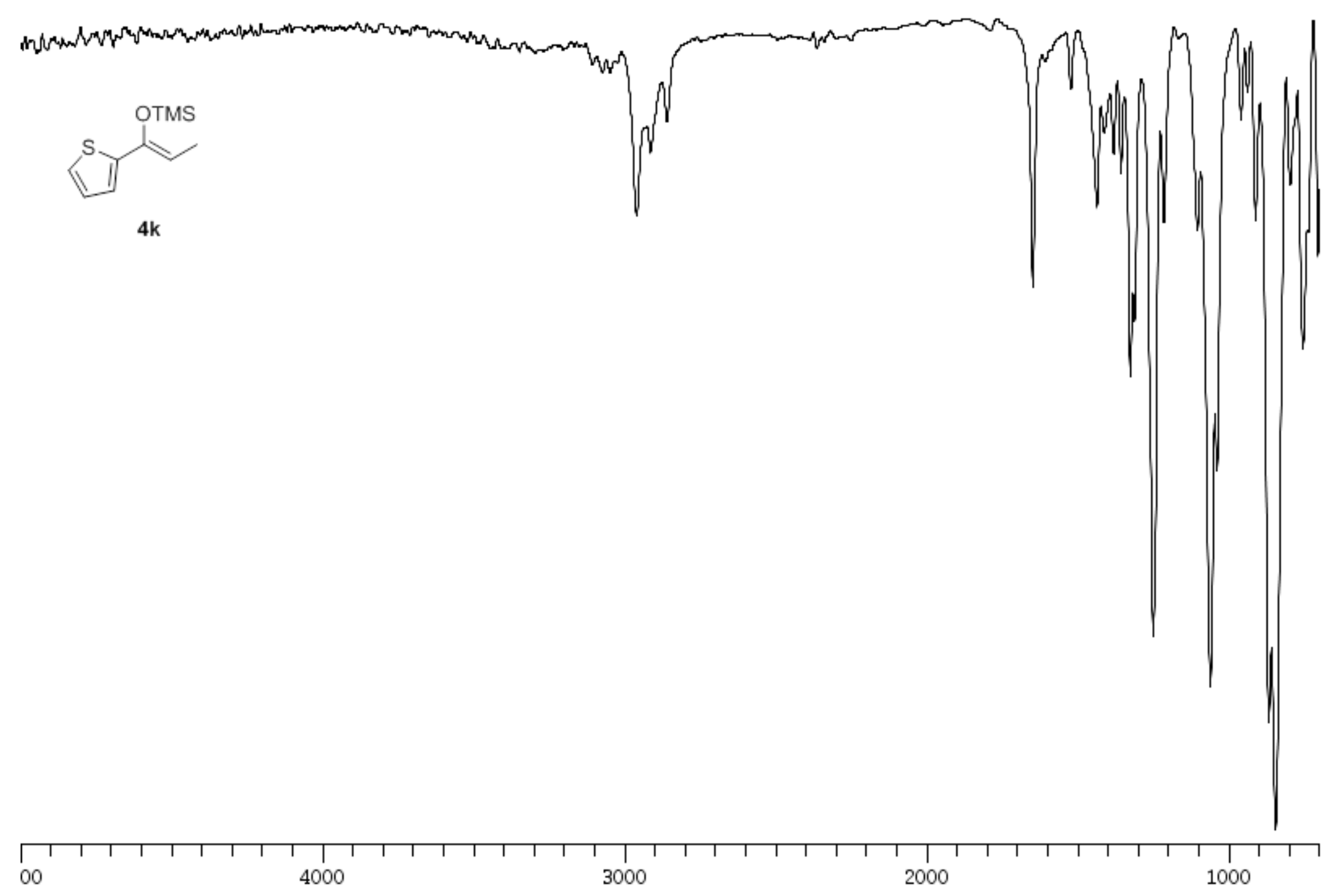




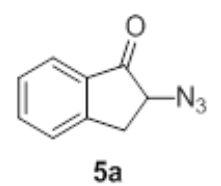

\section{${ }^{1}$ H NMR}

Solvent: $\mathbf{C D C l}_{3}$

Frequency: $400.08 \mathrm{MHz}$

$5 a$

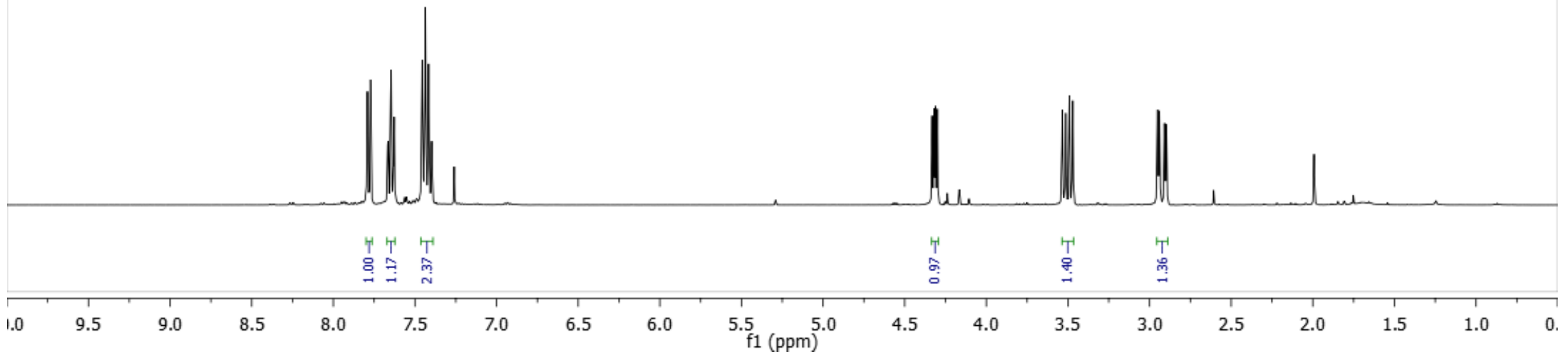




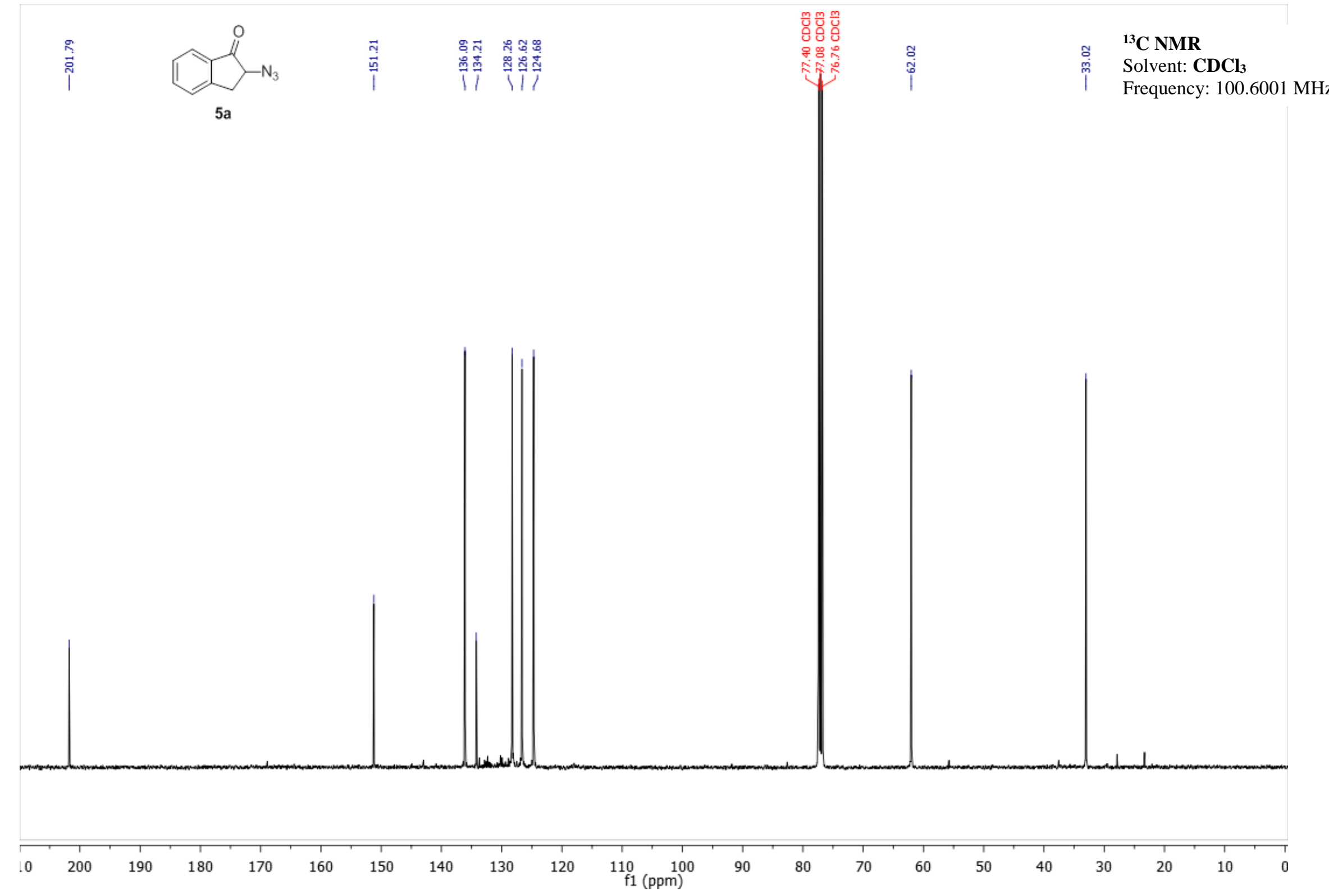




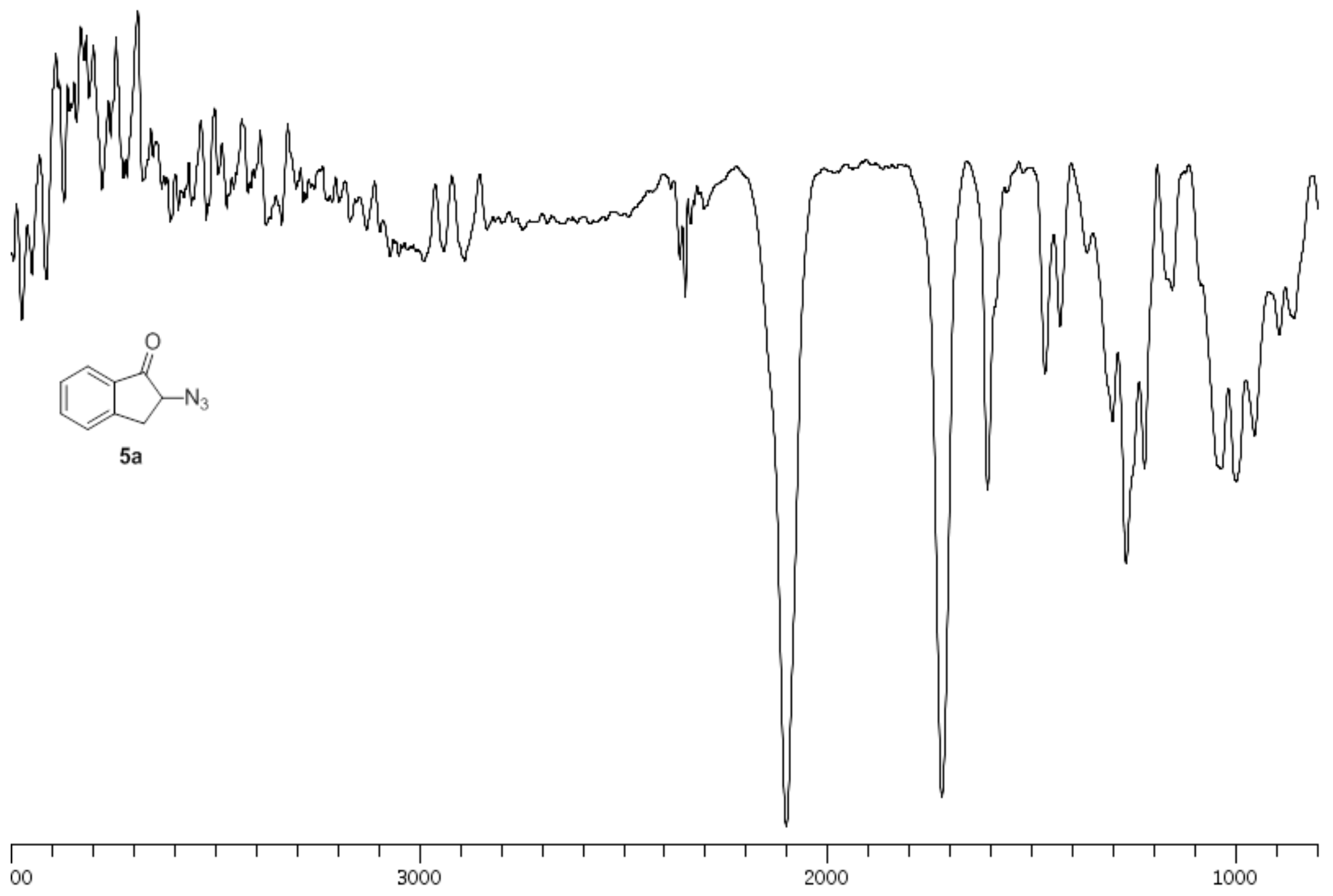




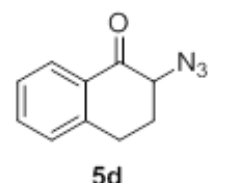

${ }^{1}$ H NMR

Solvent: $\mathbf{C D C l}_{3}$

Frequency: $400.08 \mathrm{MHz}$

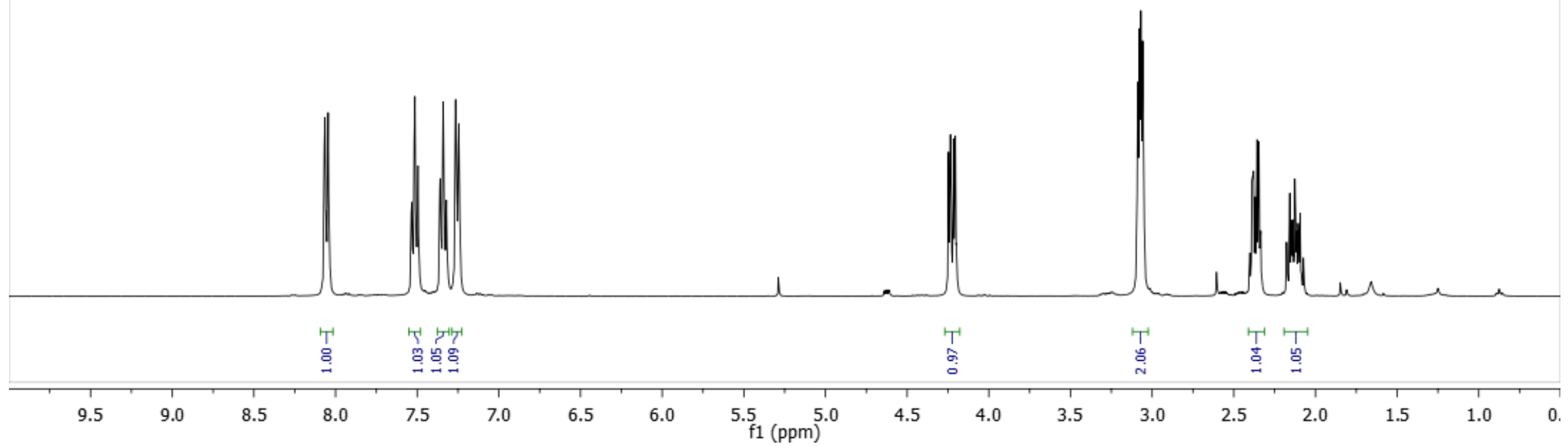




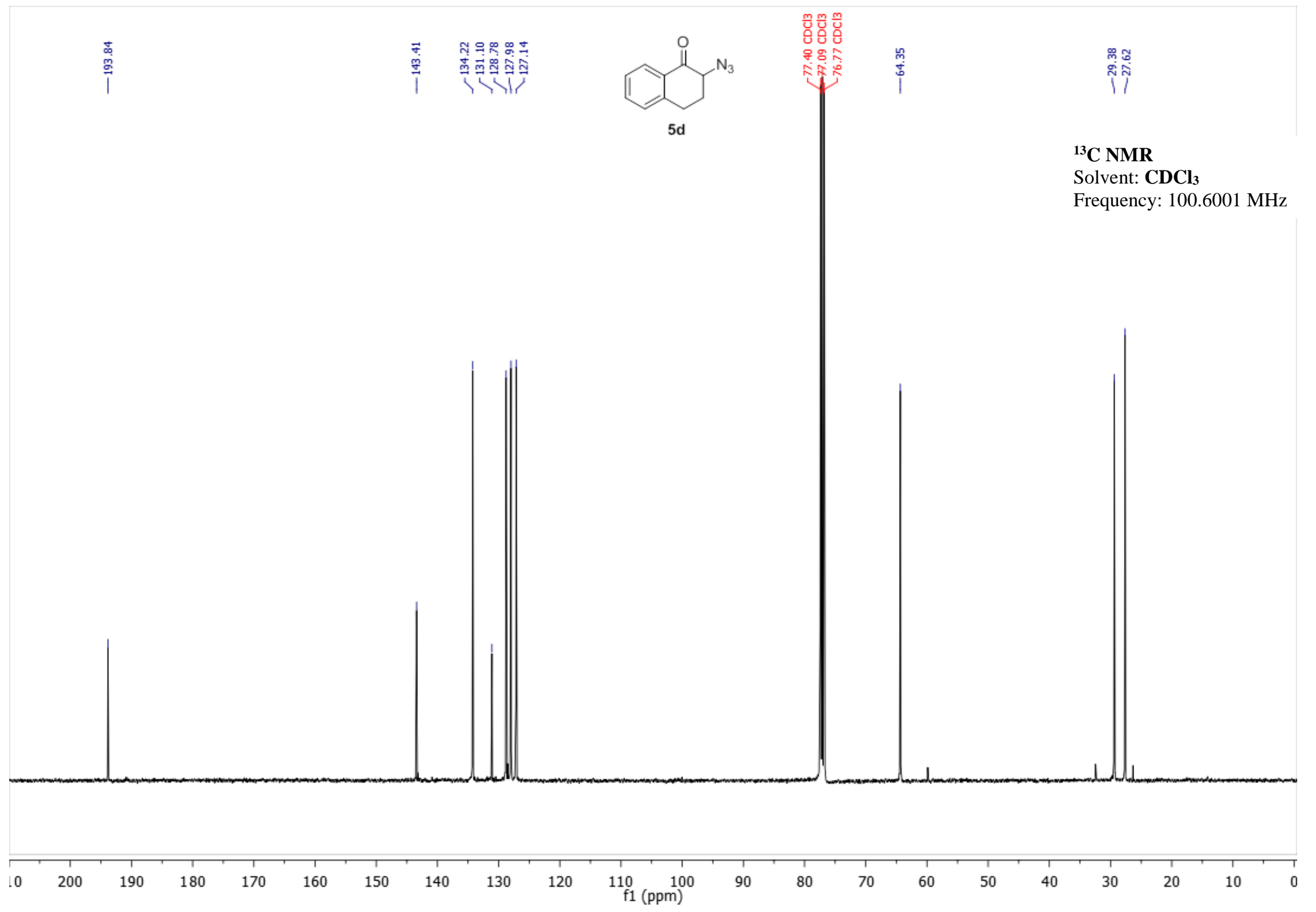




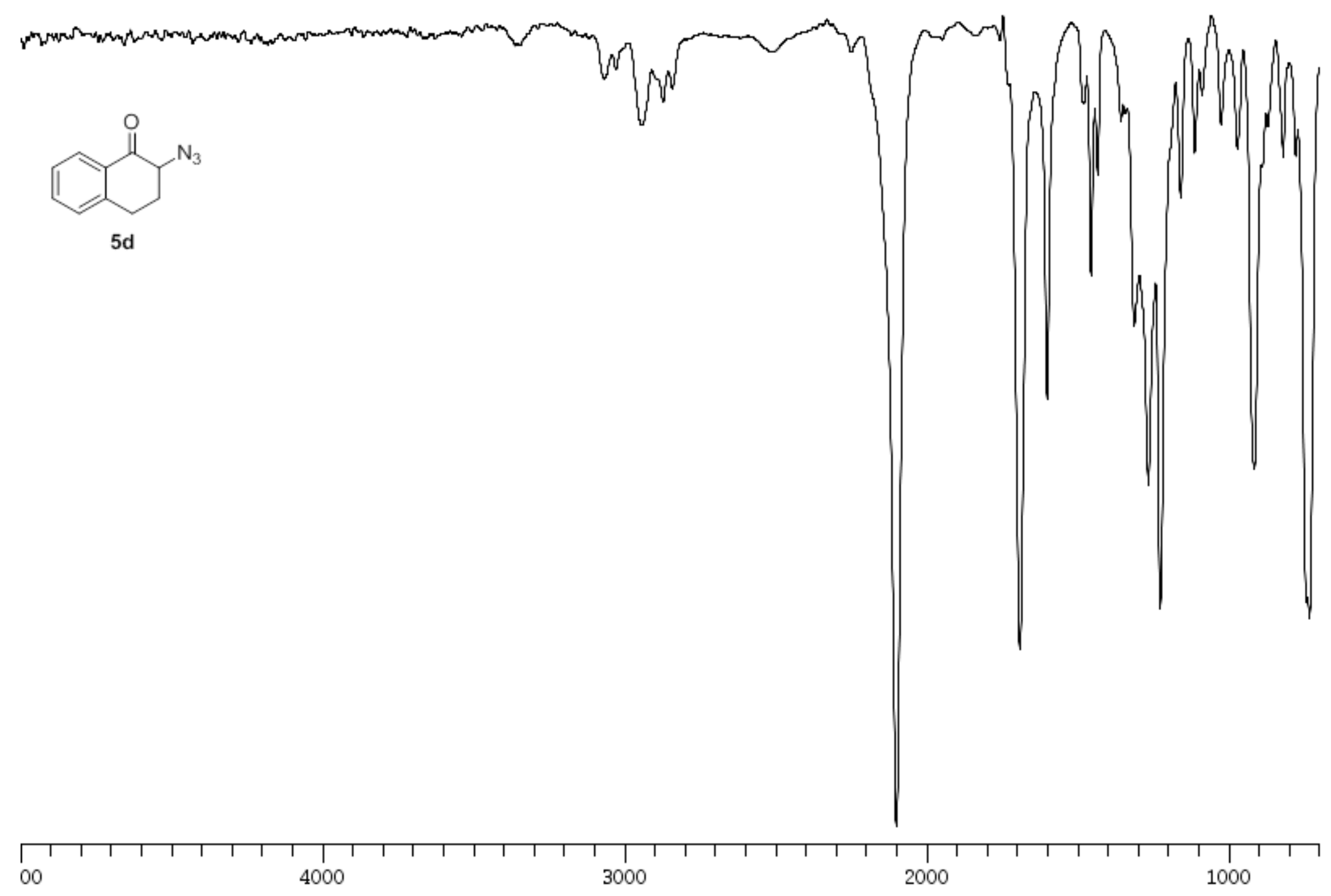




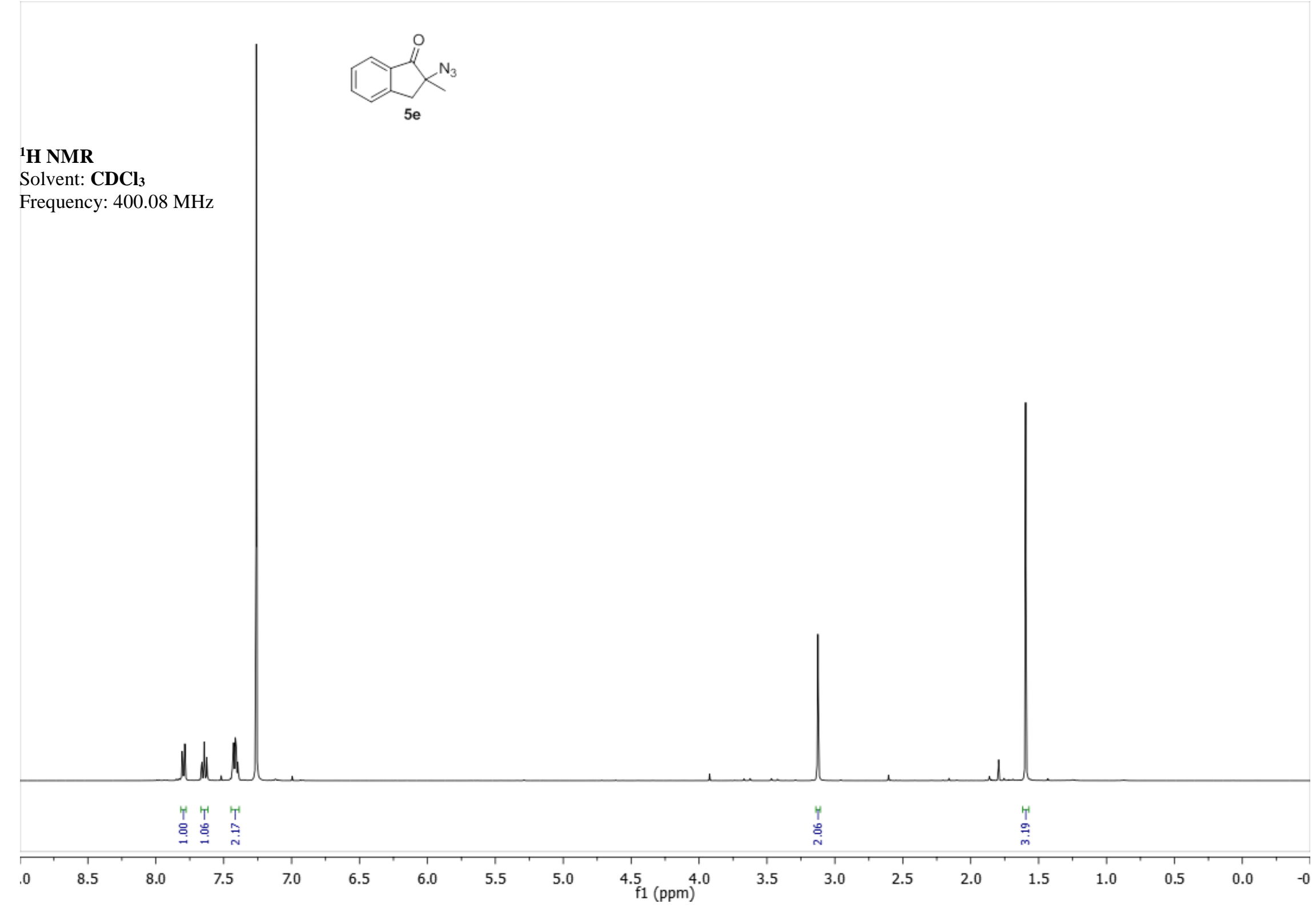




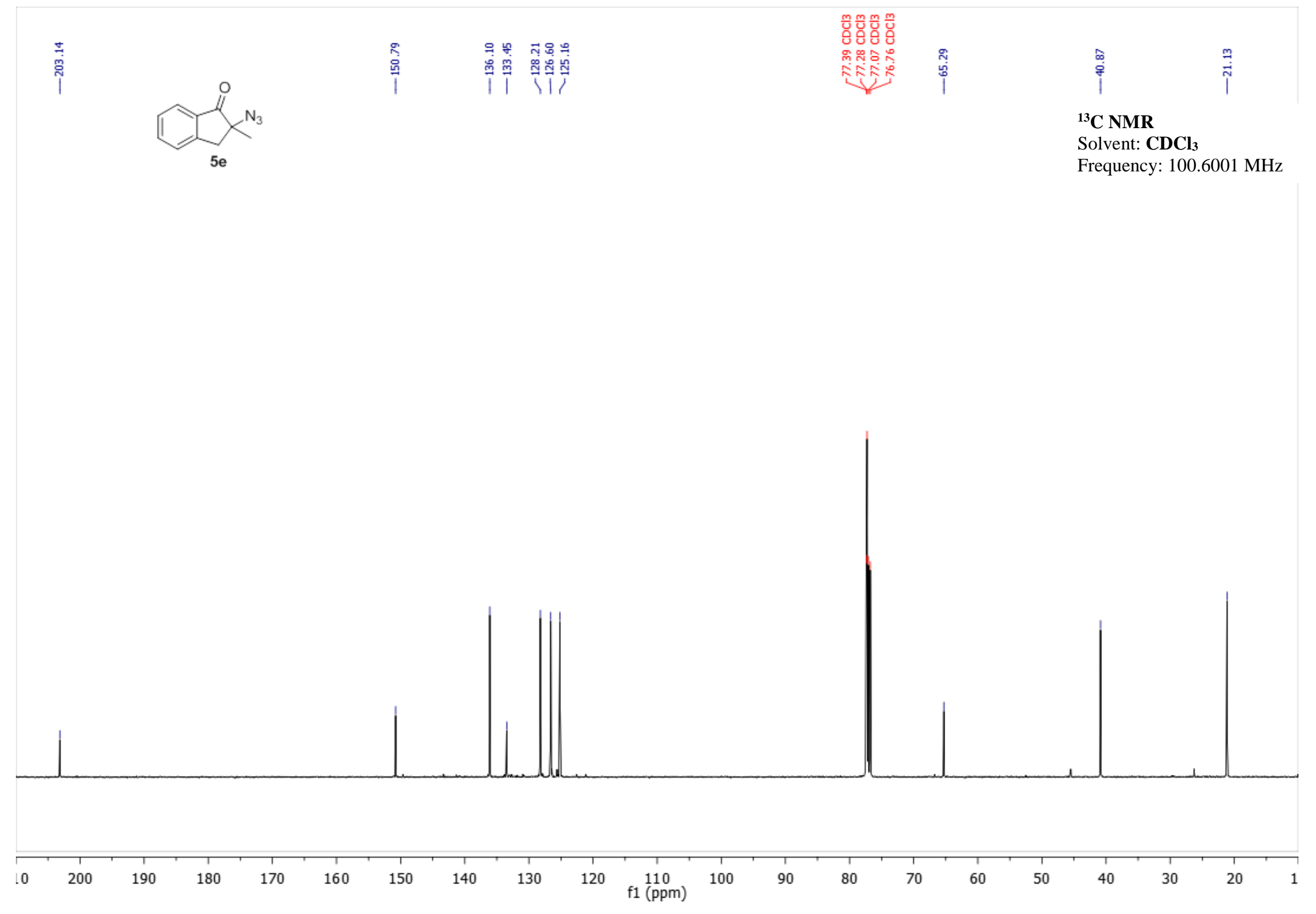




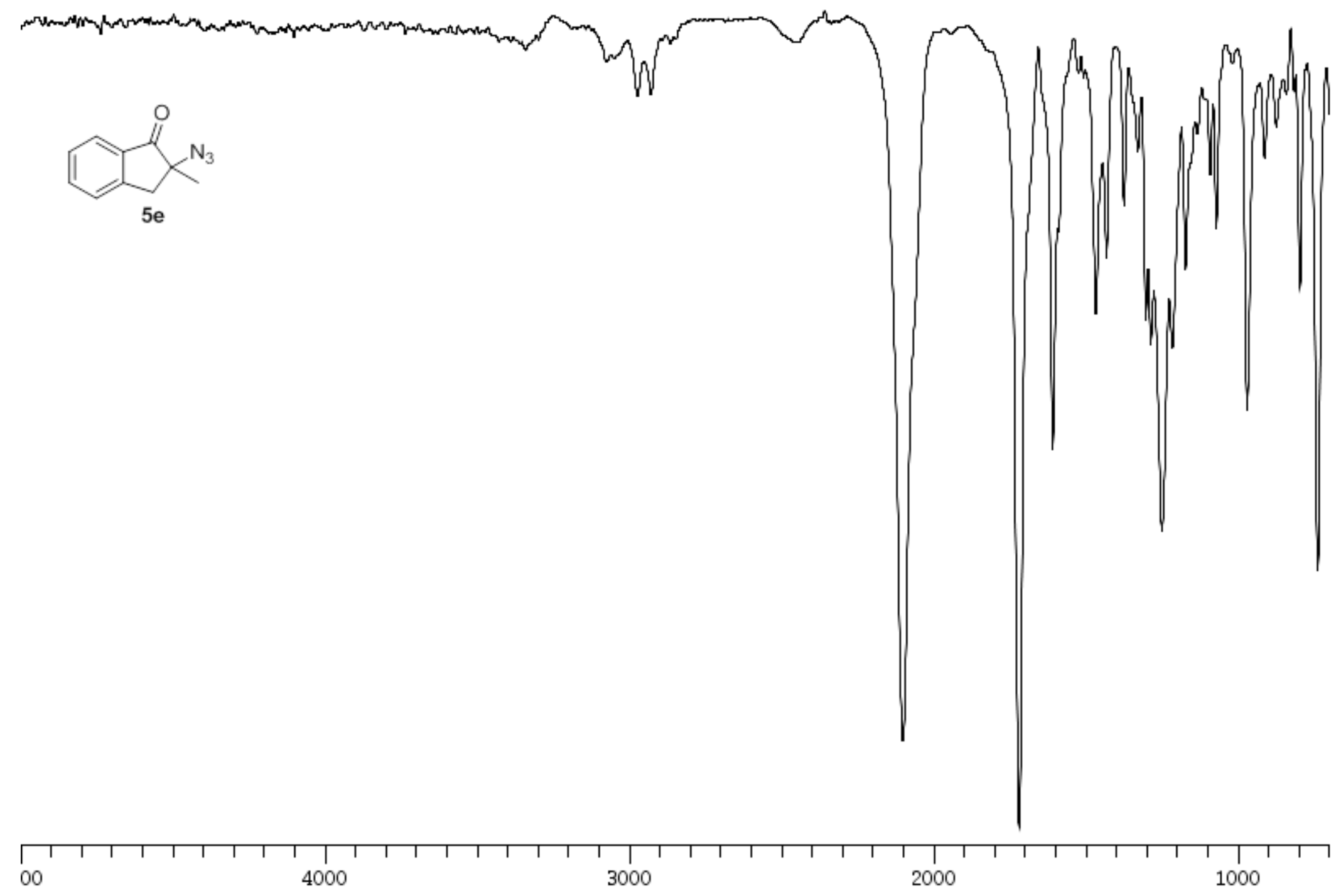




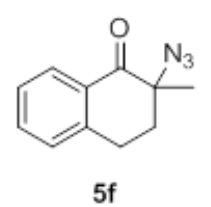

${ }^{1}$ H NMR

Solvent: $\mathbf{C D C l}_{3}$

Frequency: $400.08 \mathrm{MHz}$

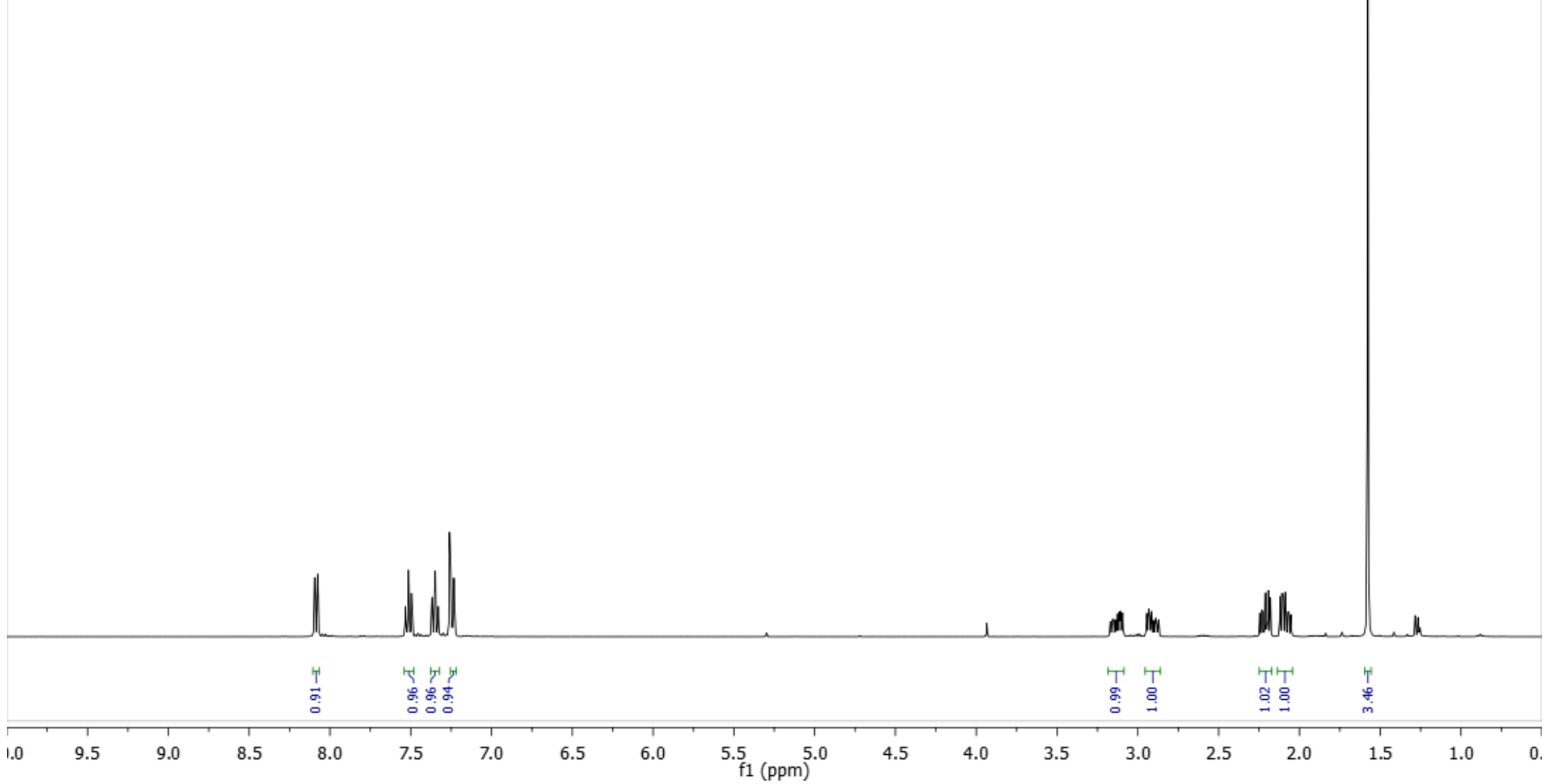




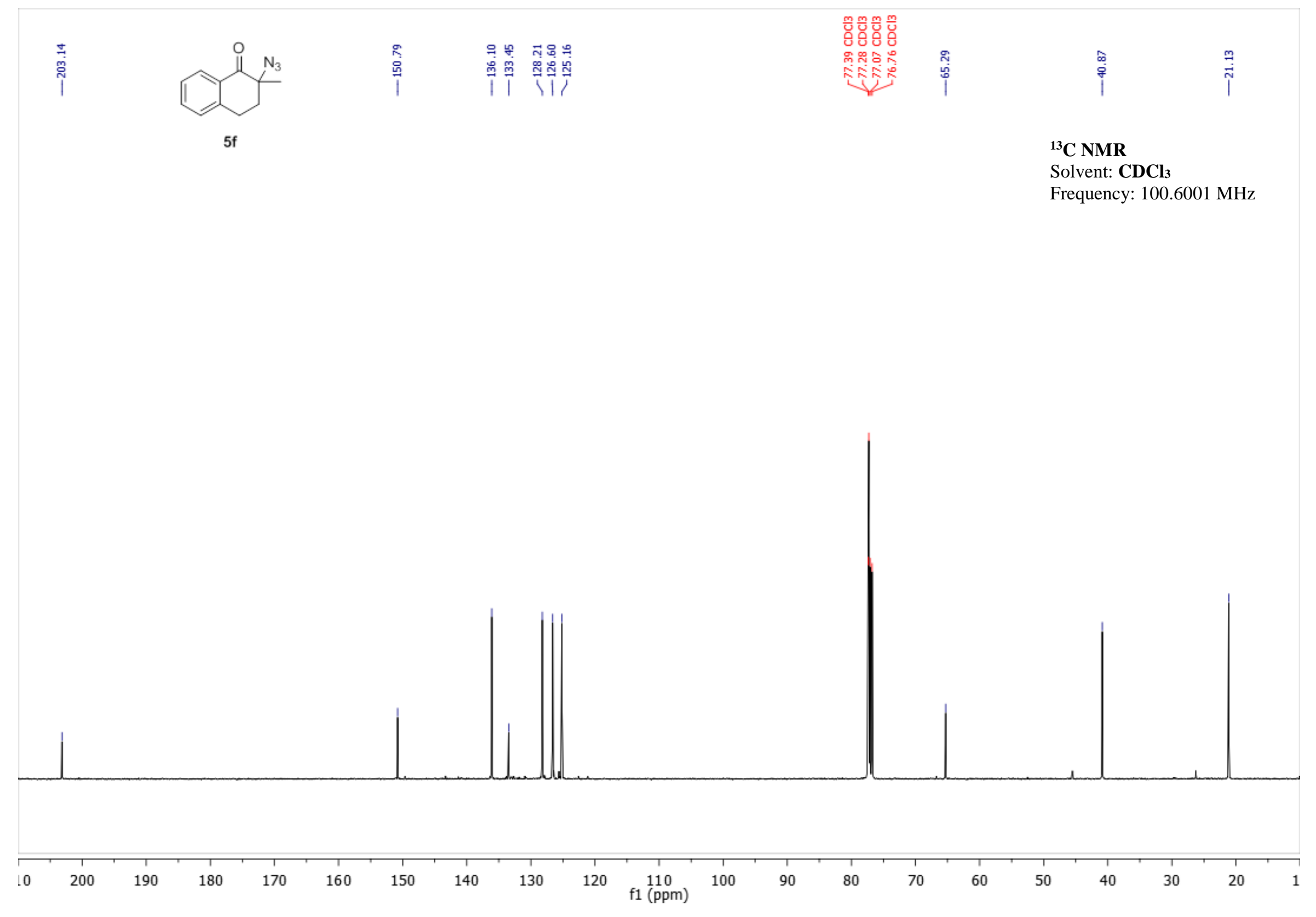




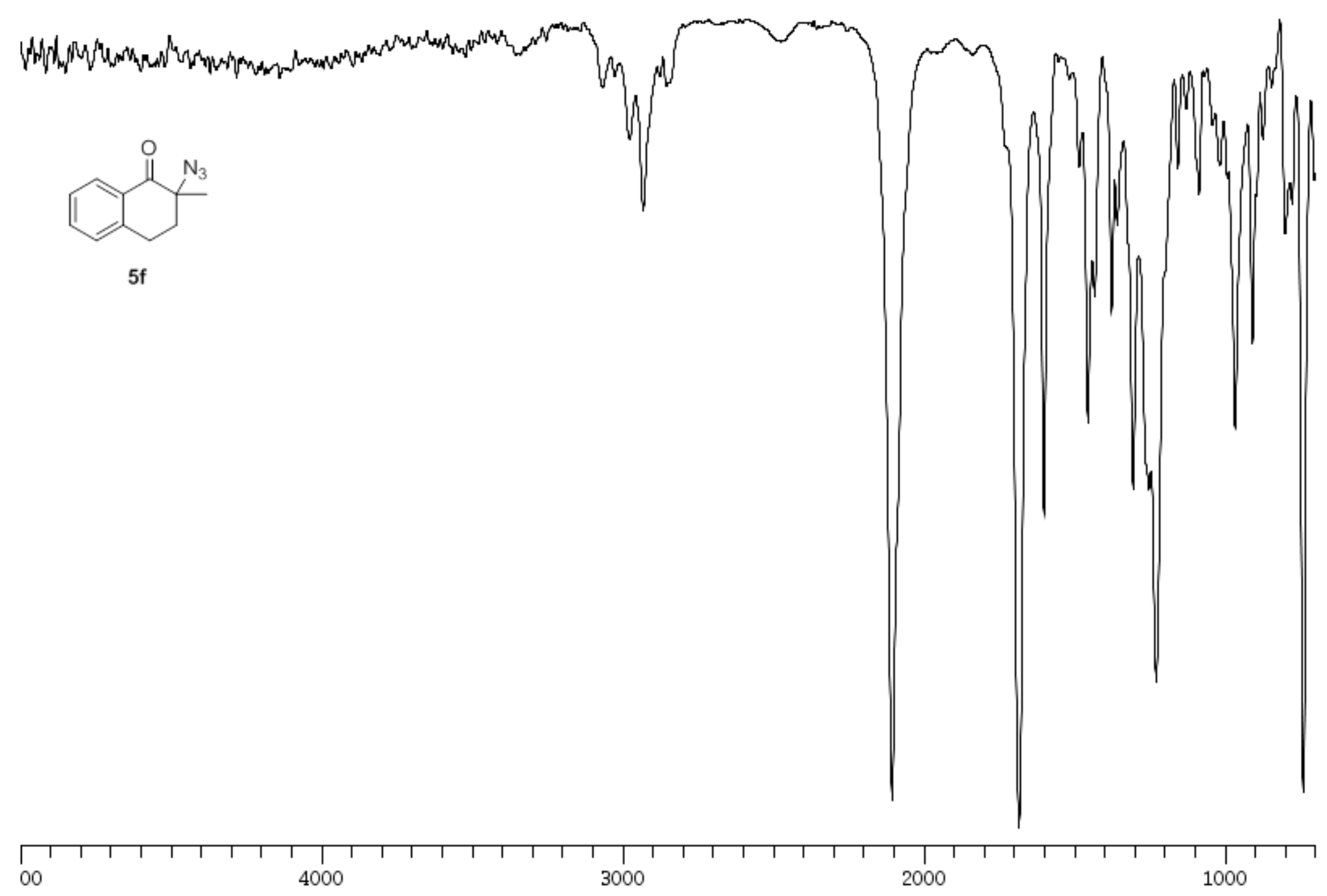




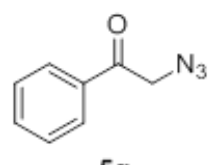

$5 \mathrm{~g}$

\section{${ }^{1}$ H NMR}

Solvent: $\mathbf{C D C l}_{3}$

Frequency: $400.08 \mathrm{MHz}$

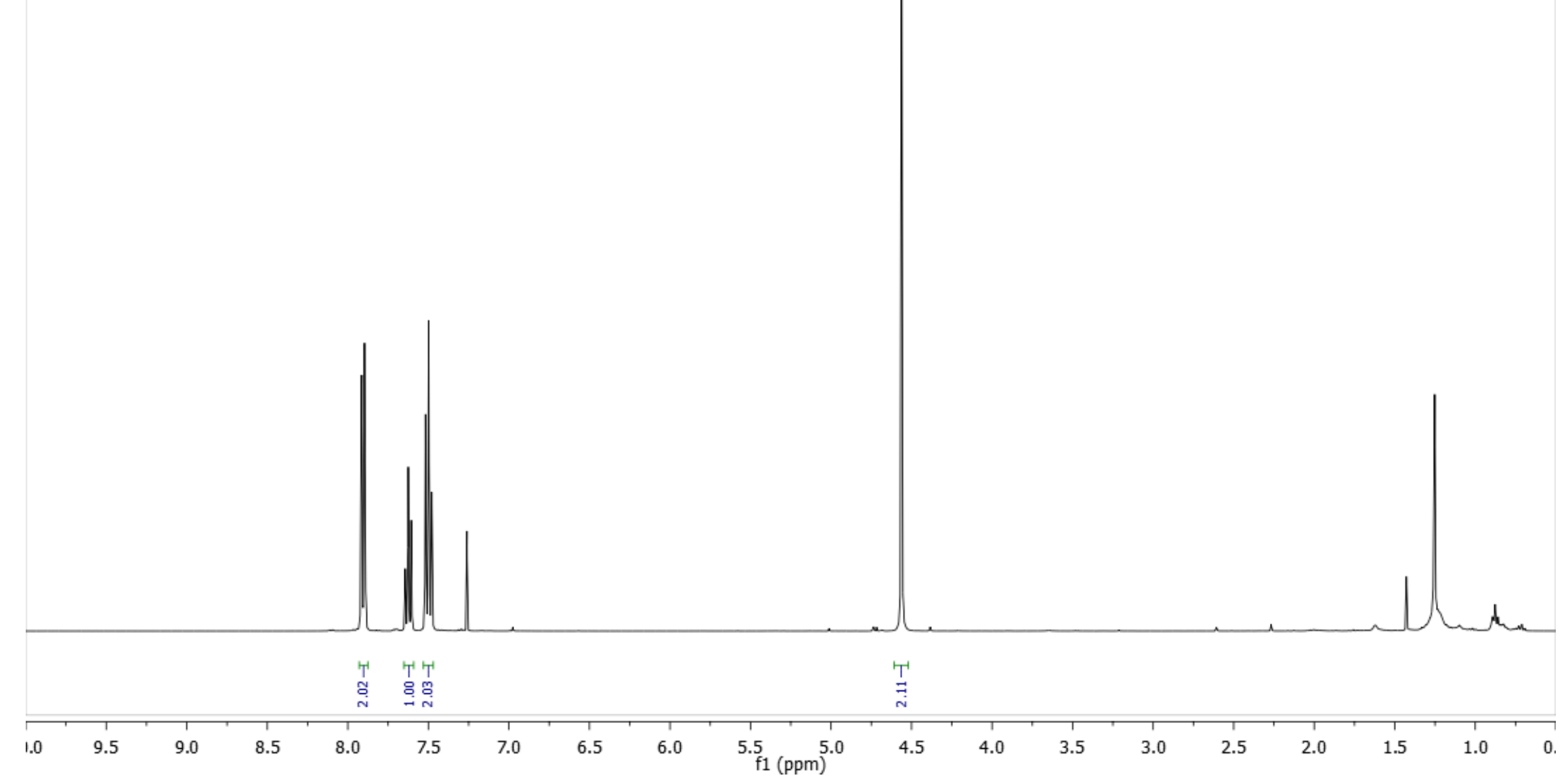




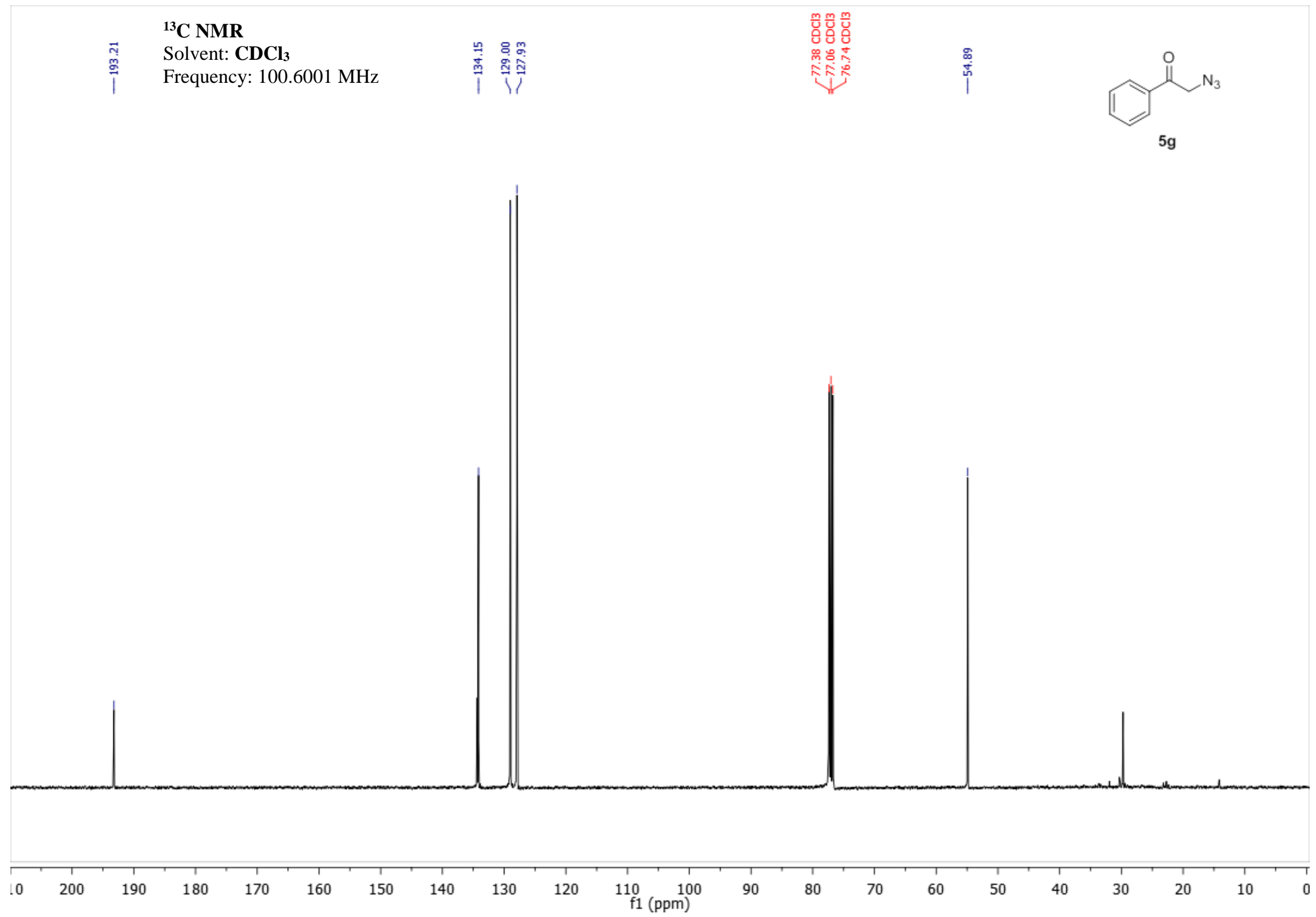

S93 
${ }^{1}$ H NMR

Solvent: $\mathbf{C D C l}_{3}$

Frequency: $400.08 \mathrm{MHz}$

$5 \mathrm{~h}$

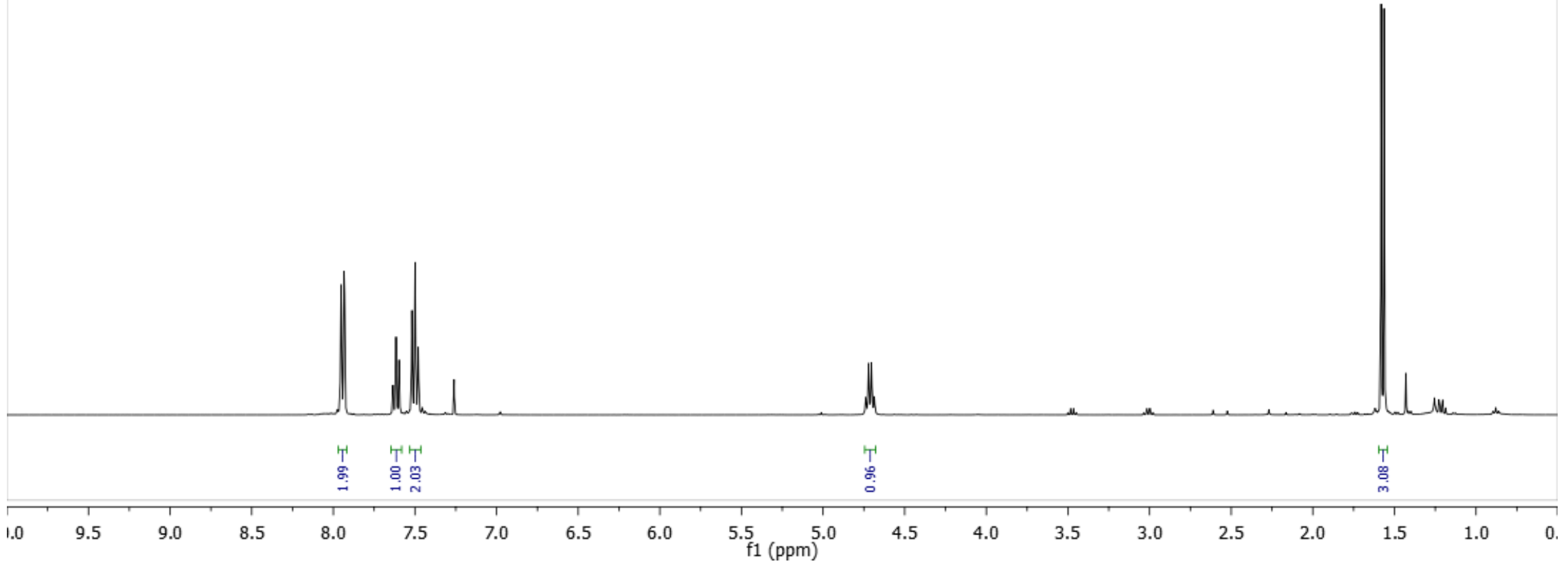




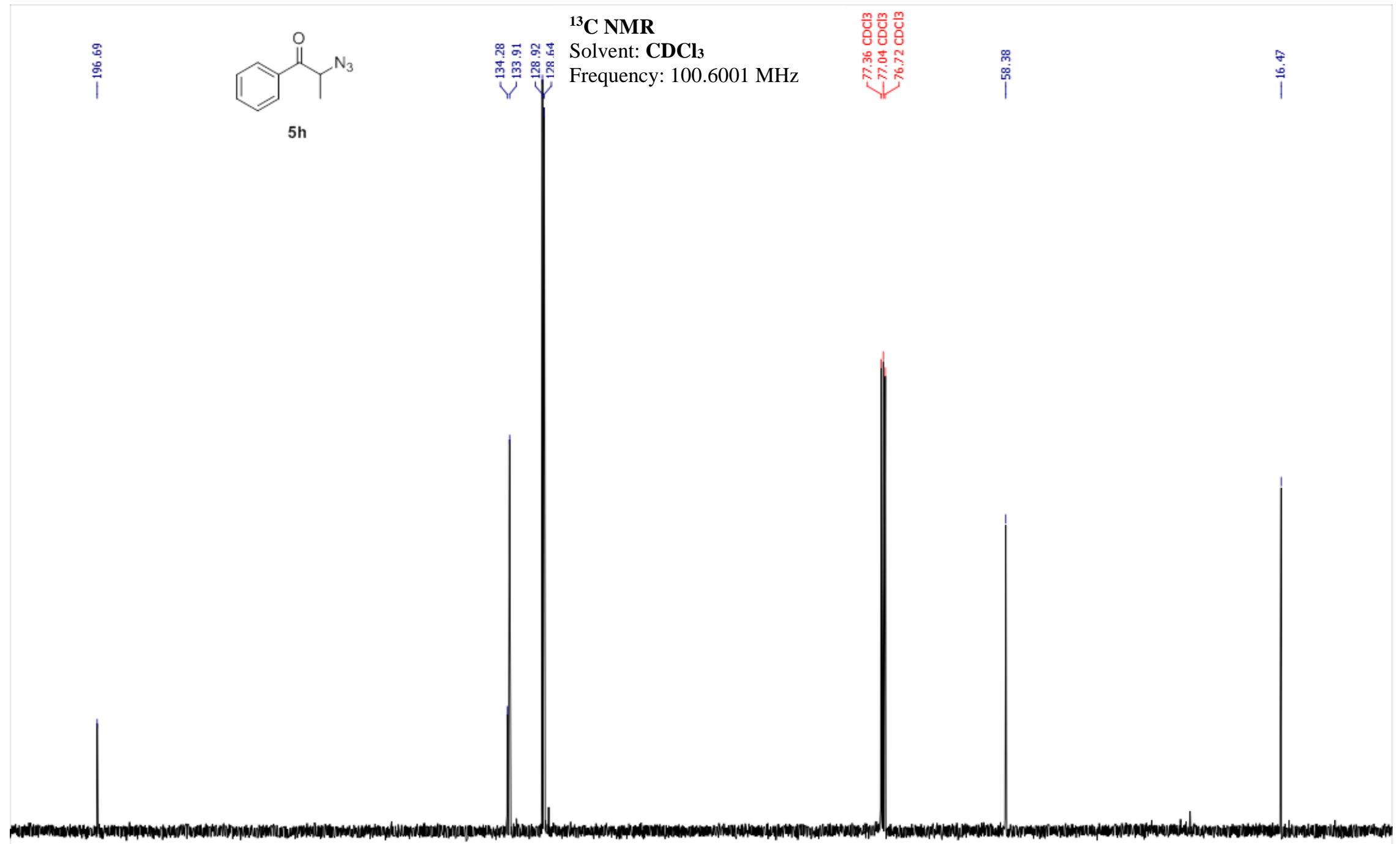




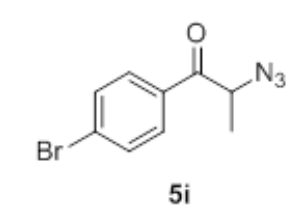

$5 i$
${ }^{1}$ H NMR

Solvent: $\mathbf{C D C l}_{3}$

Frequency: $400.08 \mathrm{MHz}$

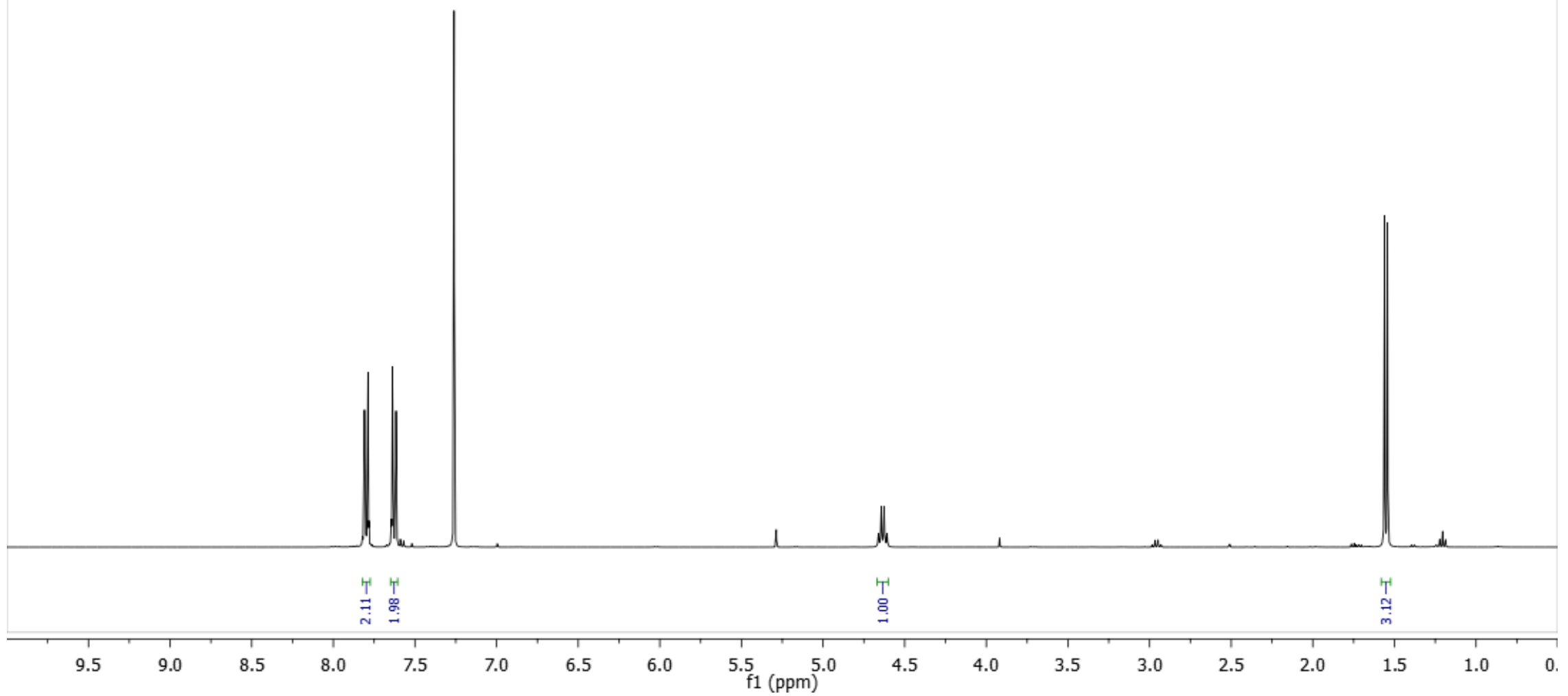




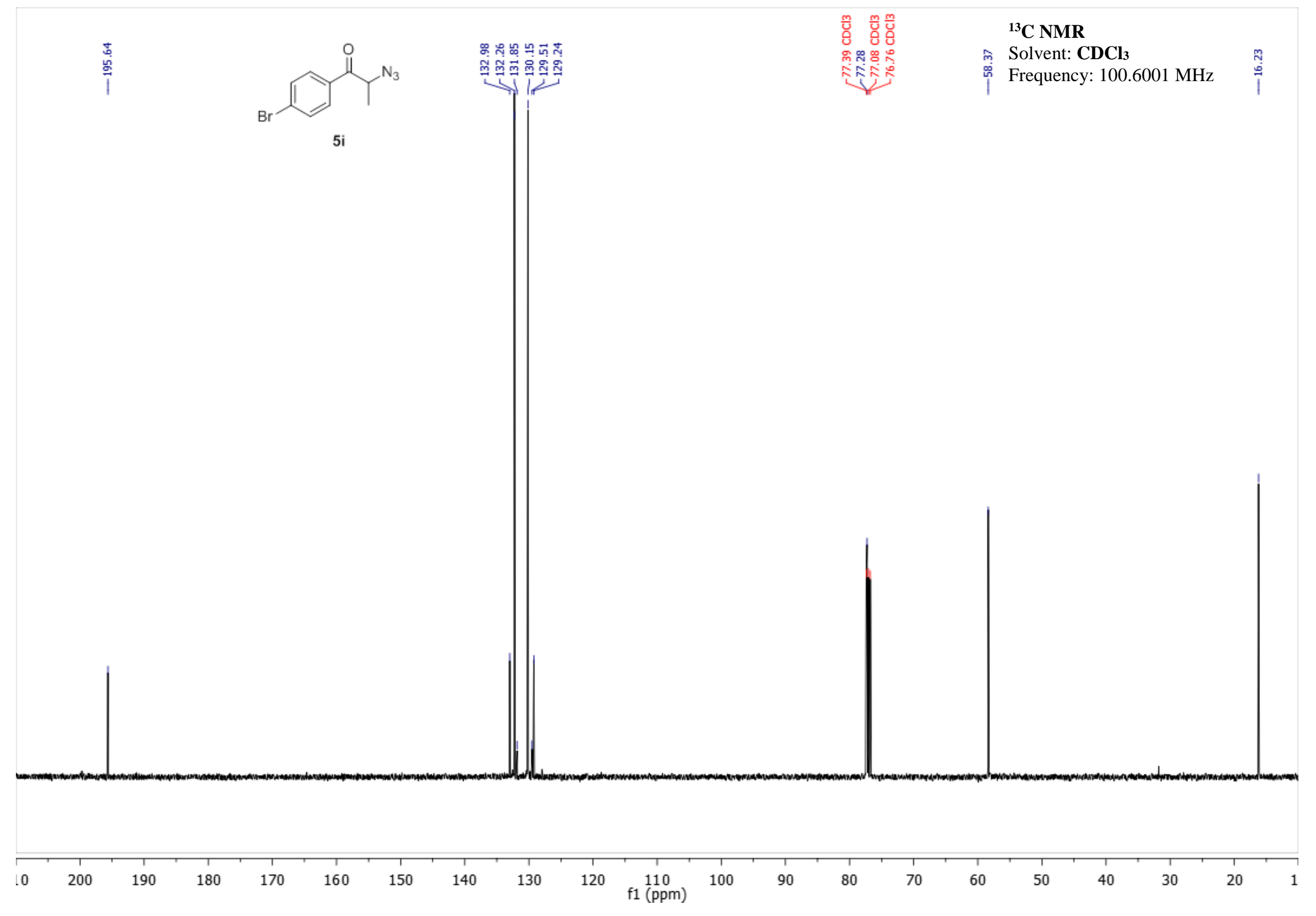




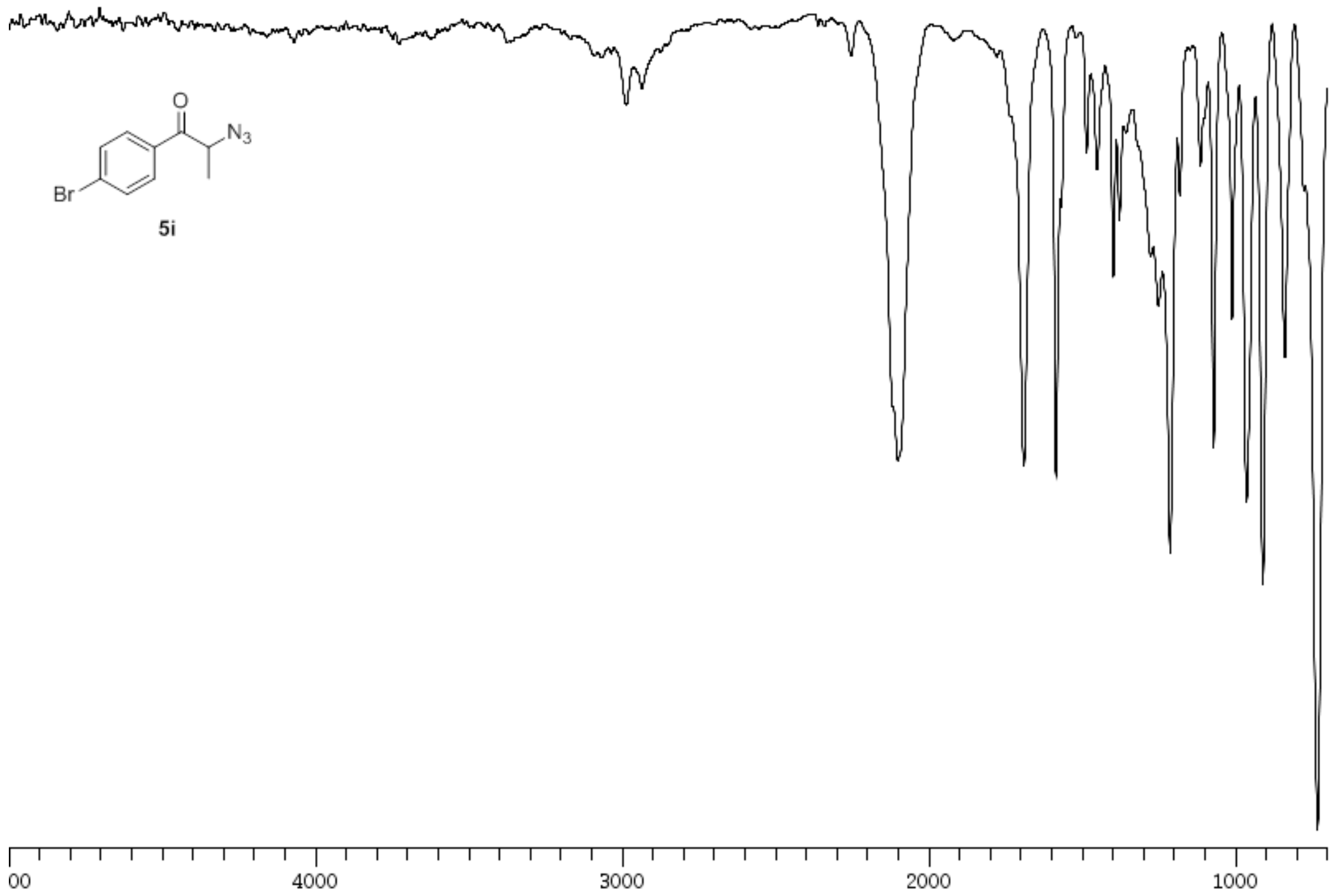




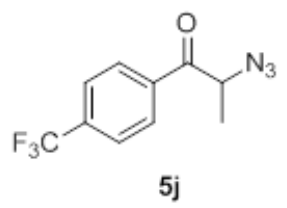

${ }^{1}$ H NMR

Solvent: $\mathbf{C D C l}_{3}$

Frequency: $400.08 \mathrm{MHz}$

5

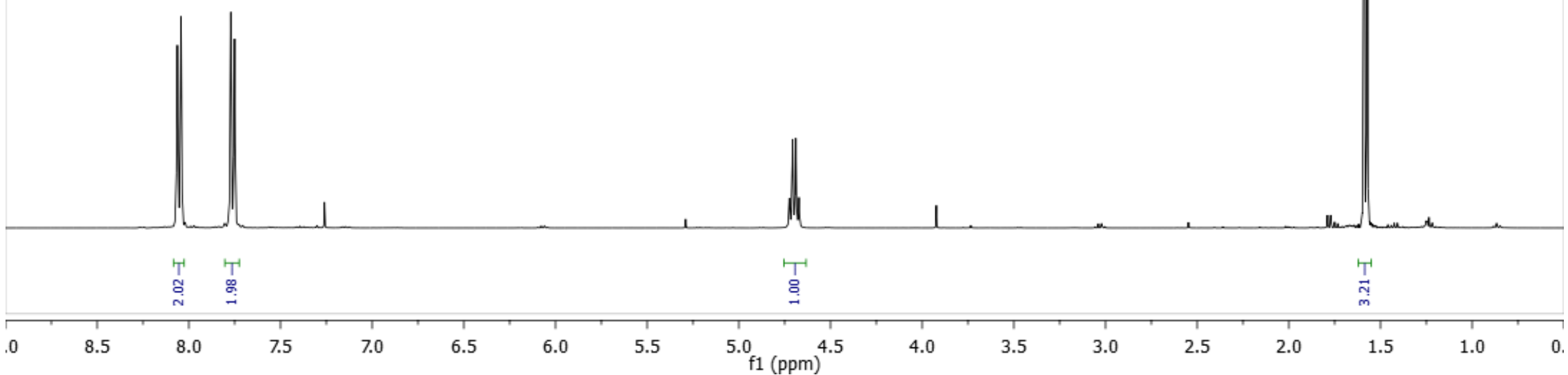




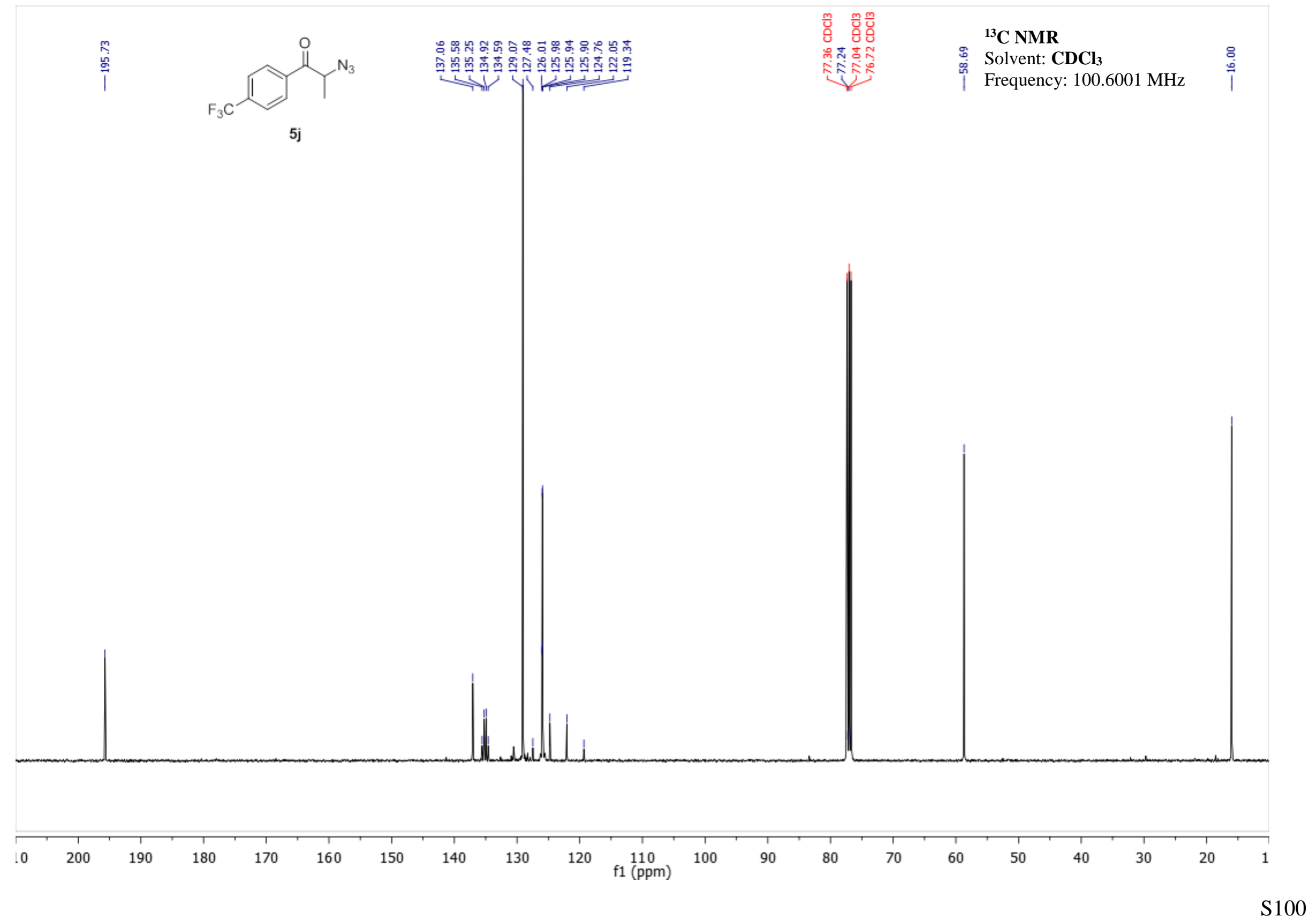




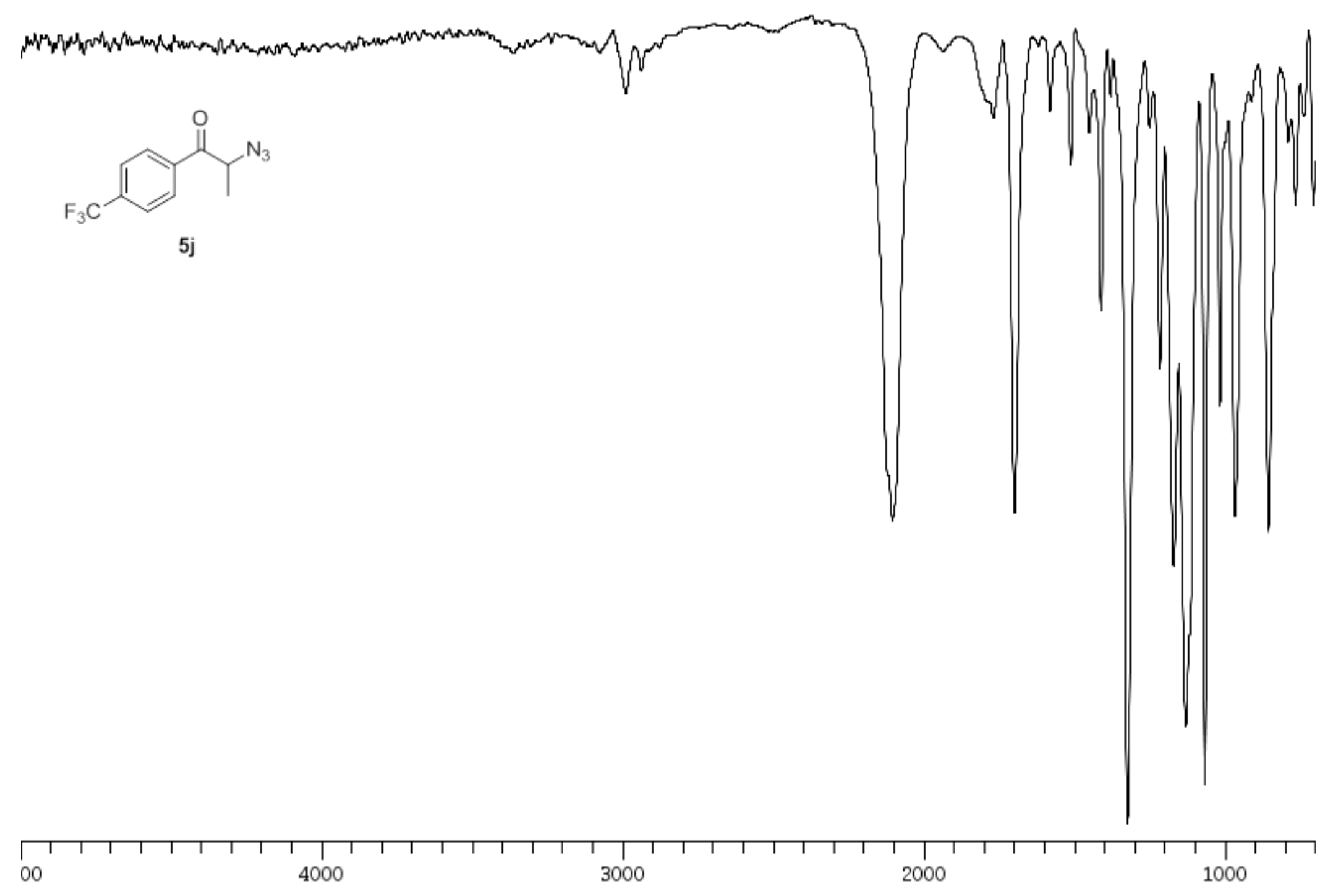




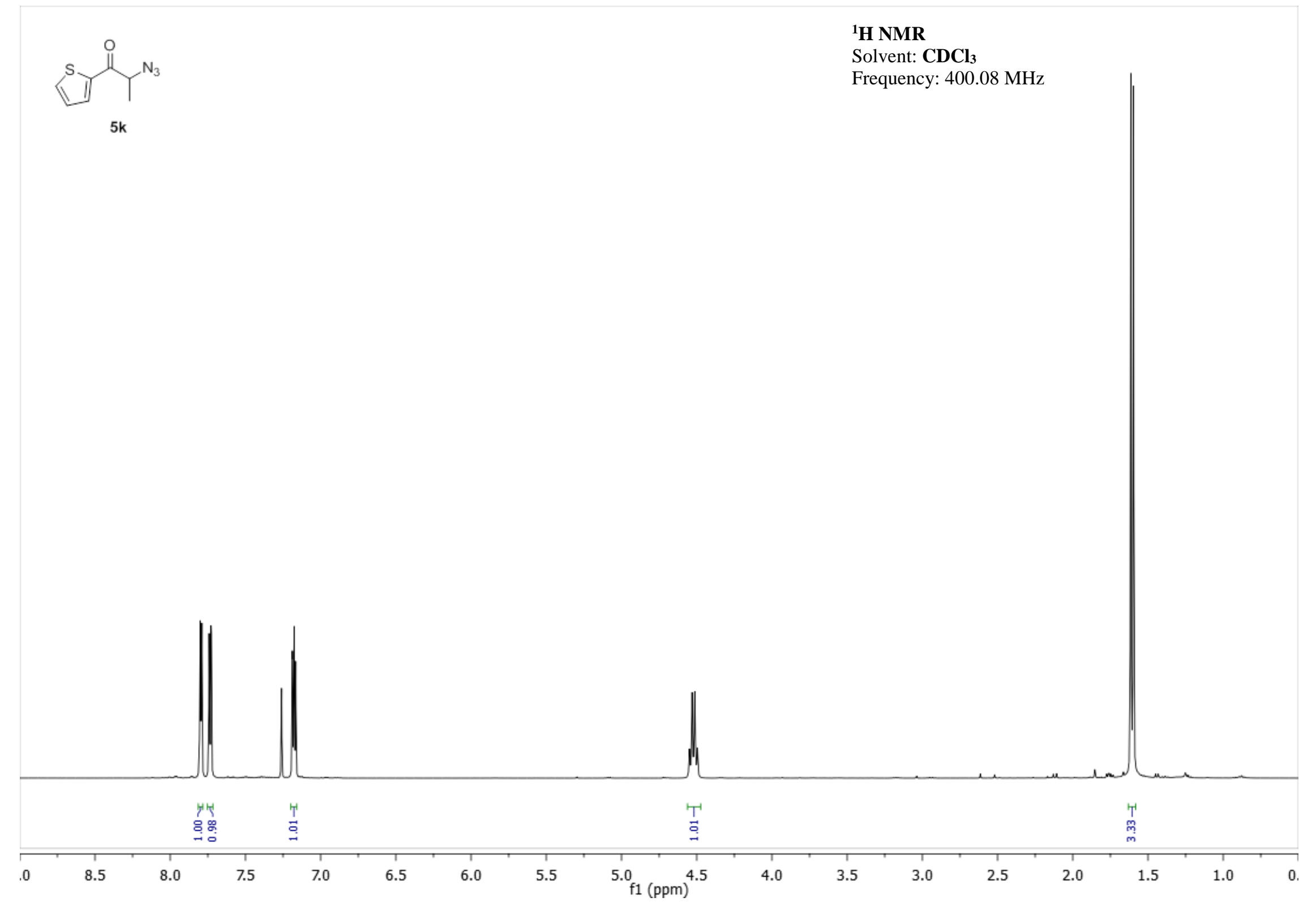




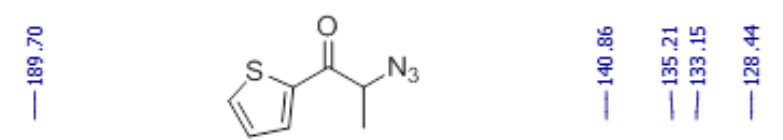

\section{${ }^{13}$ C NMR}

Solvent: $\mathbf{C D C l}_{\mathbf{3}}$

Frequency: $100.6001 \mathrm{MHz}$

$5 k$

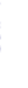

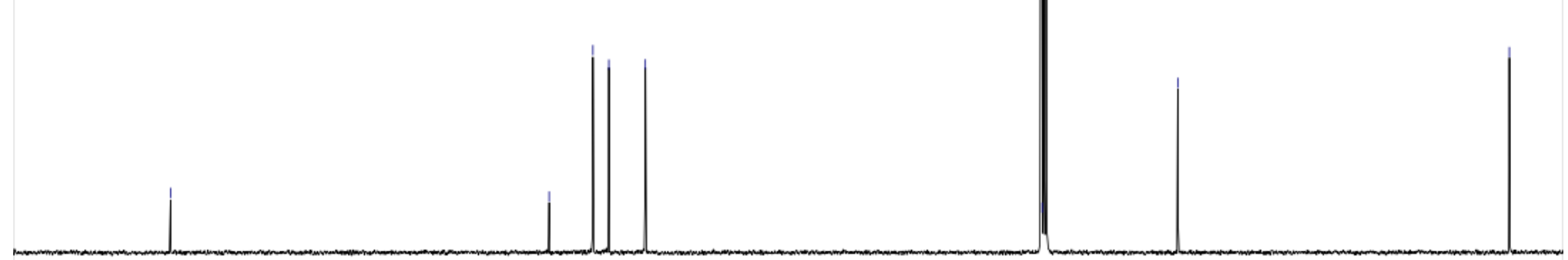

\begin{tabular}{|c|c|c|c|c|c|c|c|c|c|c|c|c|c|c|c|c|c|c|c|}
\hline .0 & 200 & 190 & 180 & $\begin{array}{l}1 \\
170\end{array}$ & $\begin{array}{c}1 \\
160\end{array}$ & 150 & 140 & 130 & 120 & $\begin{array}{c}1 \\
110\end{array}$ & 100 & 90 & 80 & 70 & 60 & 50 & 40 & 30 & 20 \\
\hline & 200 & 190 & 180 & 170 & 160 & 150 & 140 & 130 & 120 & f1 (ppm) & 100 & 90 & 80 & 70 & 60 & 50 & 40 & 30 & 20 \\
\hline
\end{tabular}




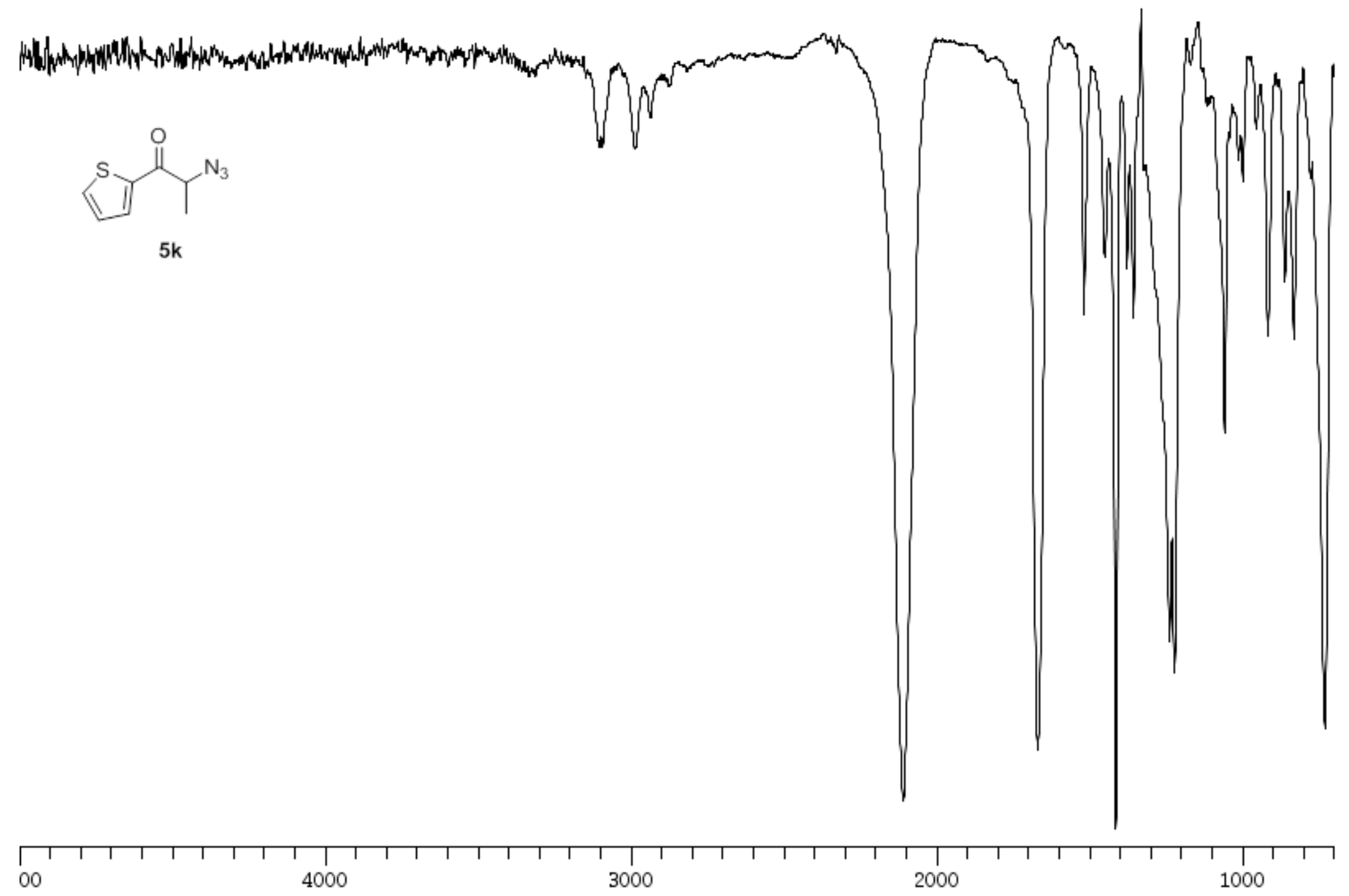




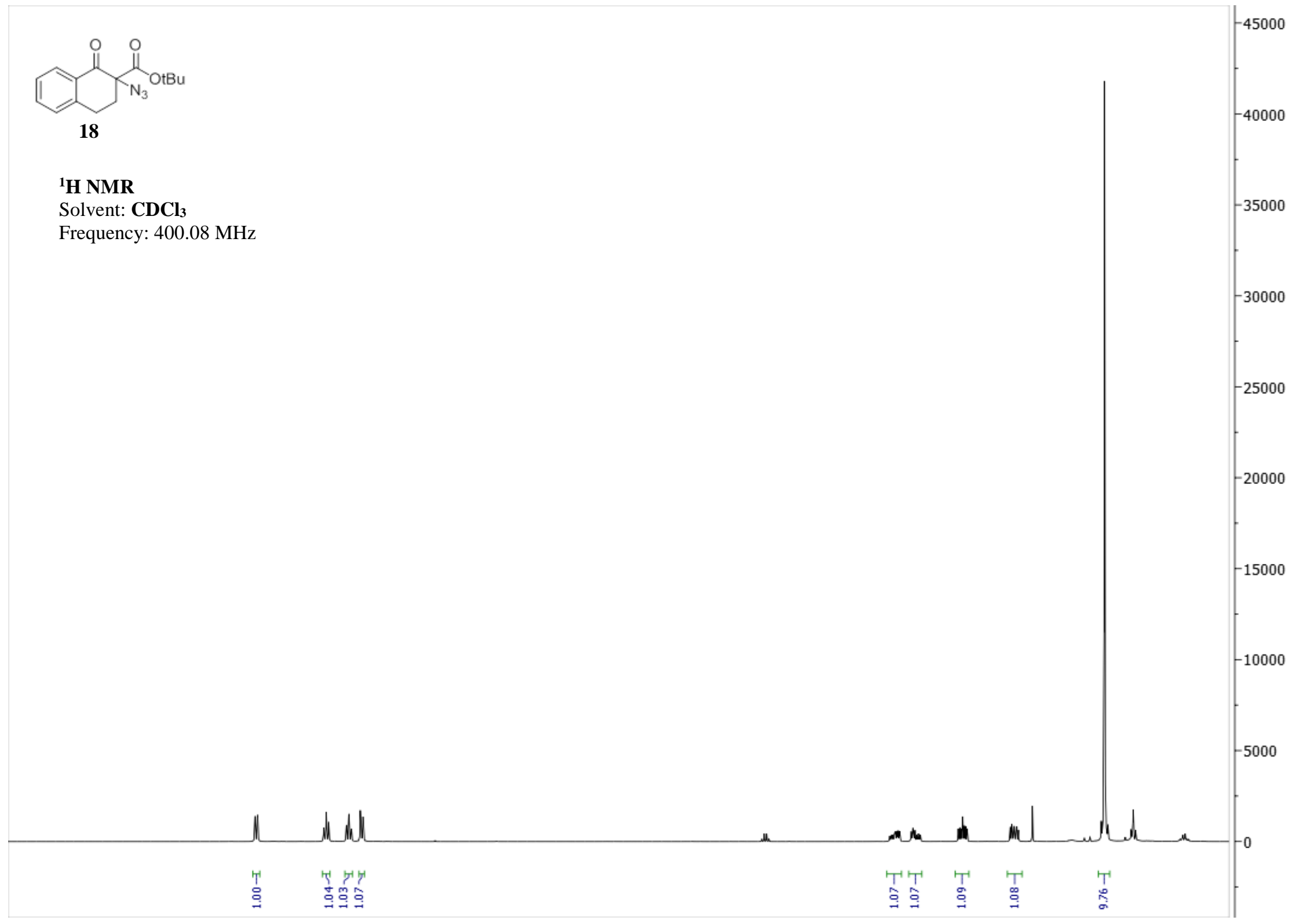




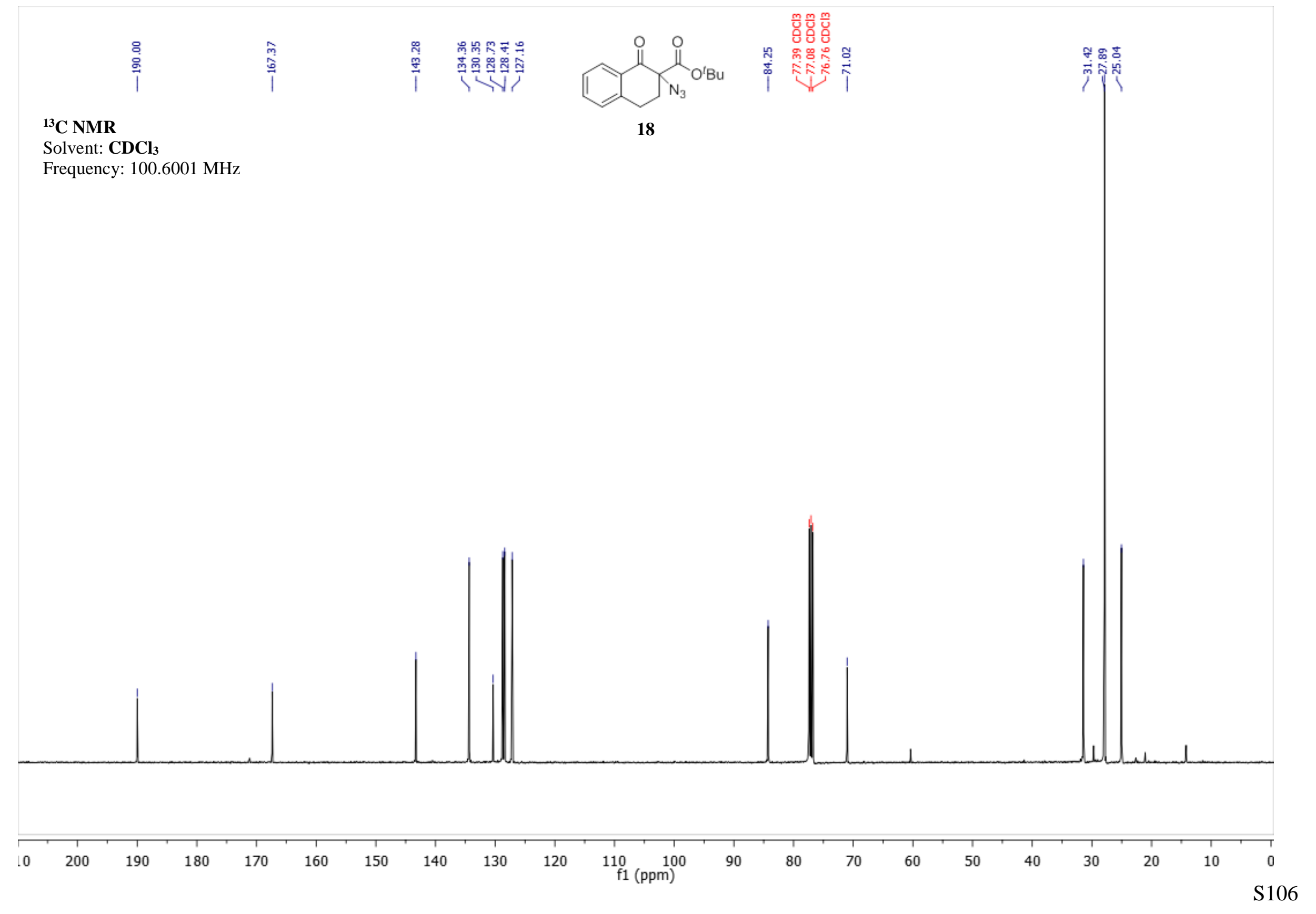




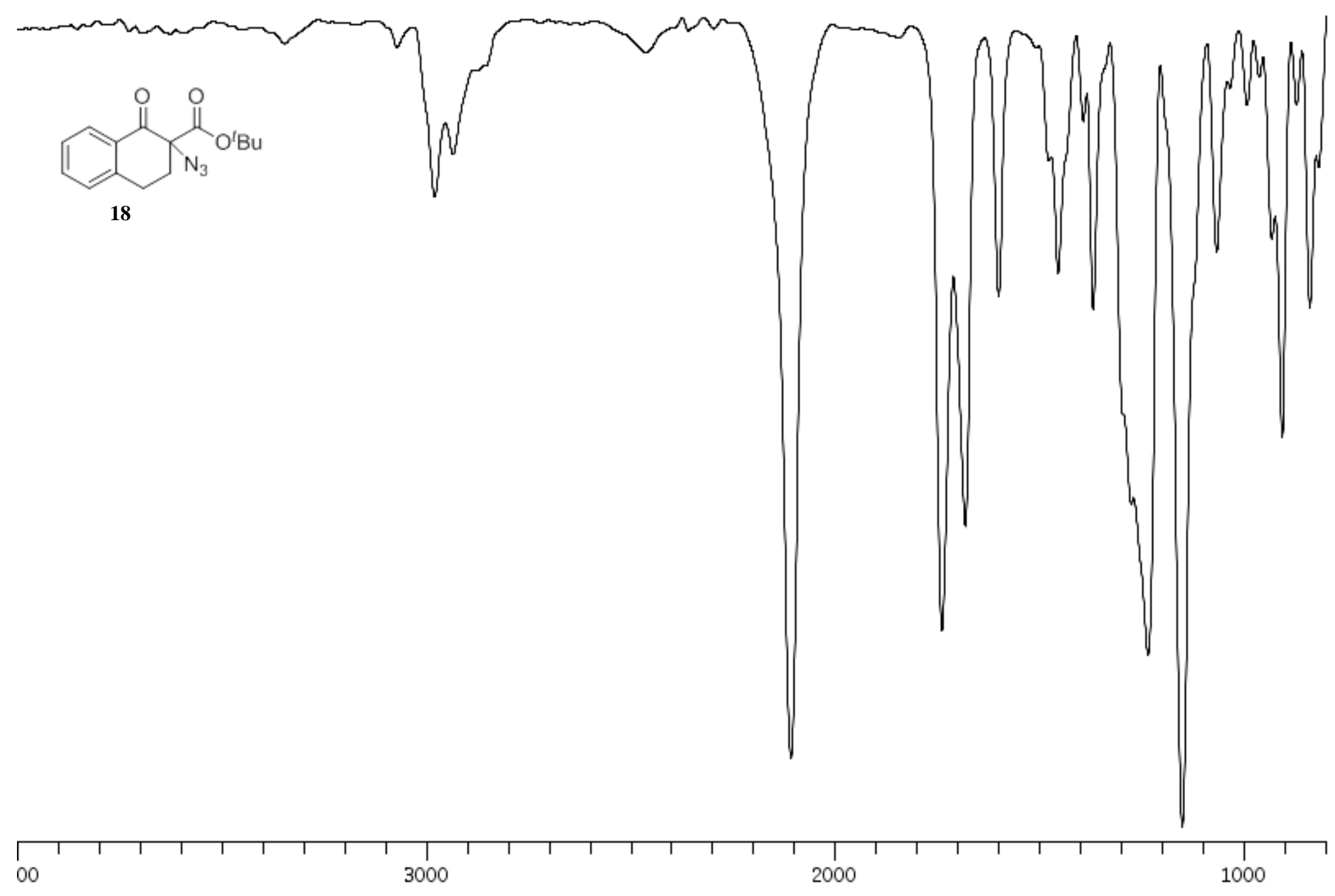




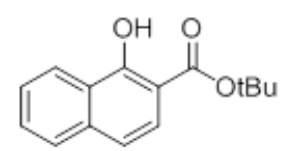

19

${ }^{1}$ H NMR

Solvent: $\mathbf{C D C l}_{3}$

Frequency: $400.08 \mathrm{MHz}$

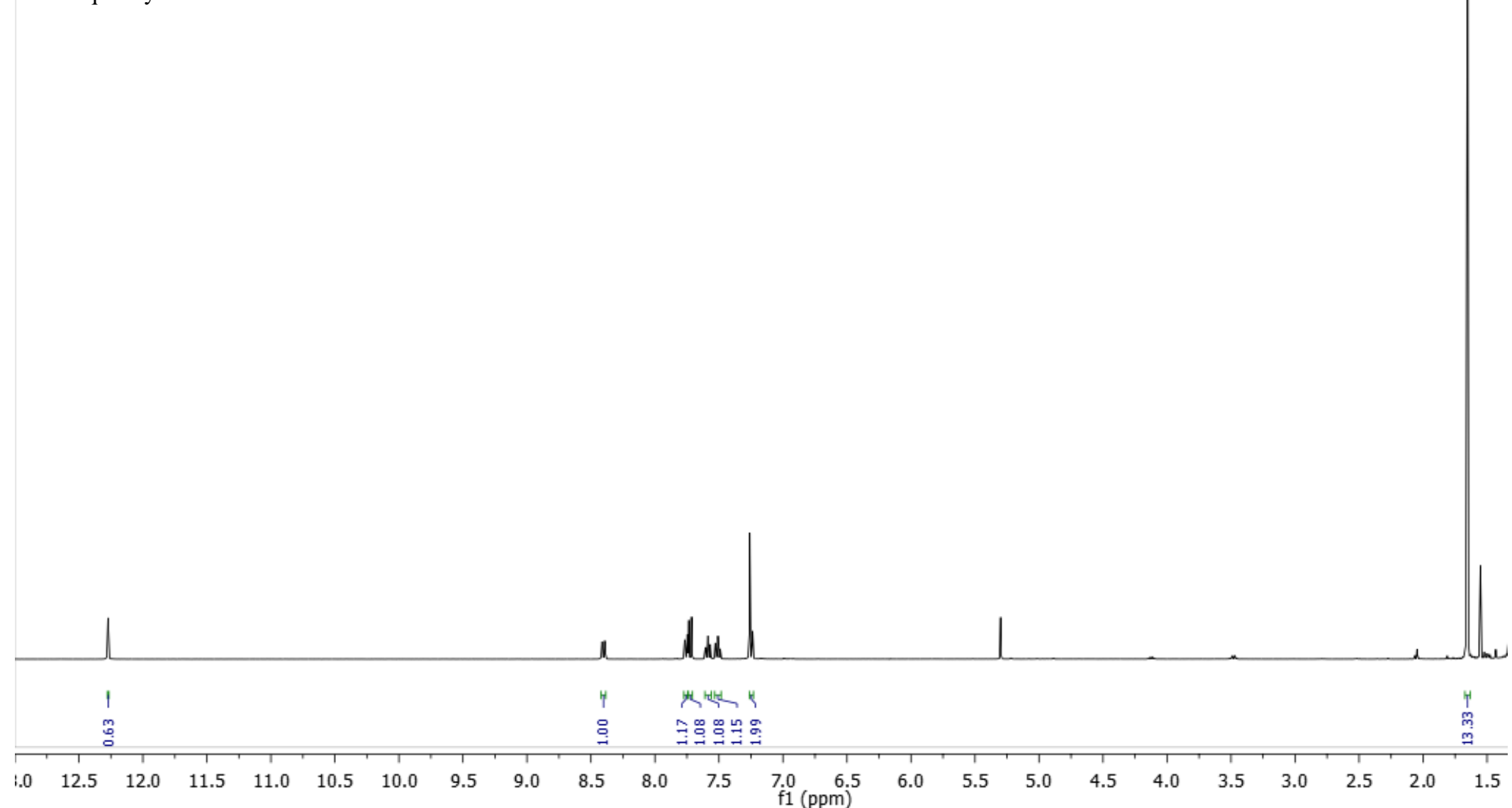




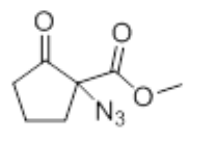

20

\section{${ }^{1}$ H NMR}

Solvent: $\mathbf{C D C l}_{\mathbf{3}}$

Frequency: $400.08 \mathrm{MHz}$

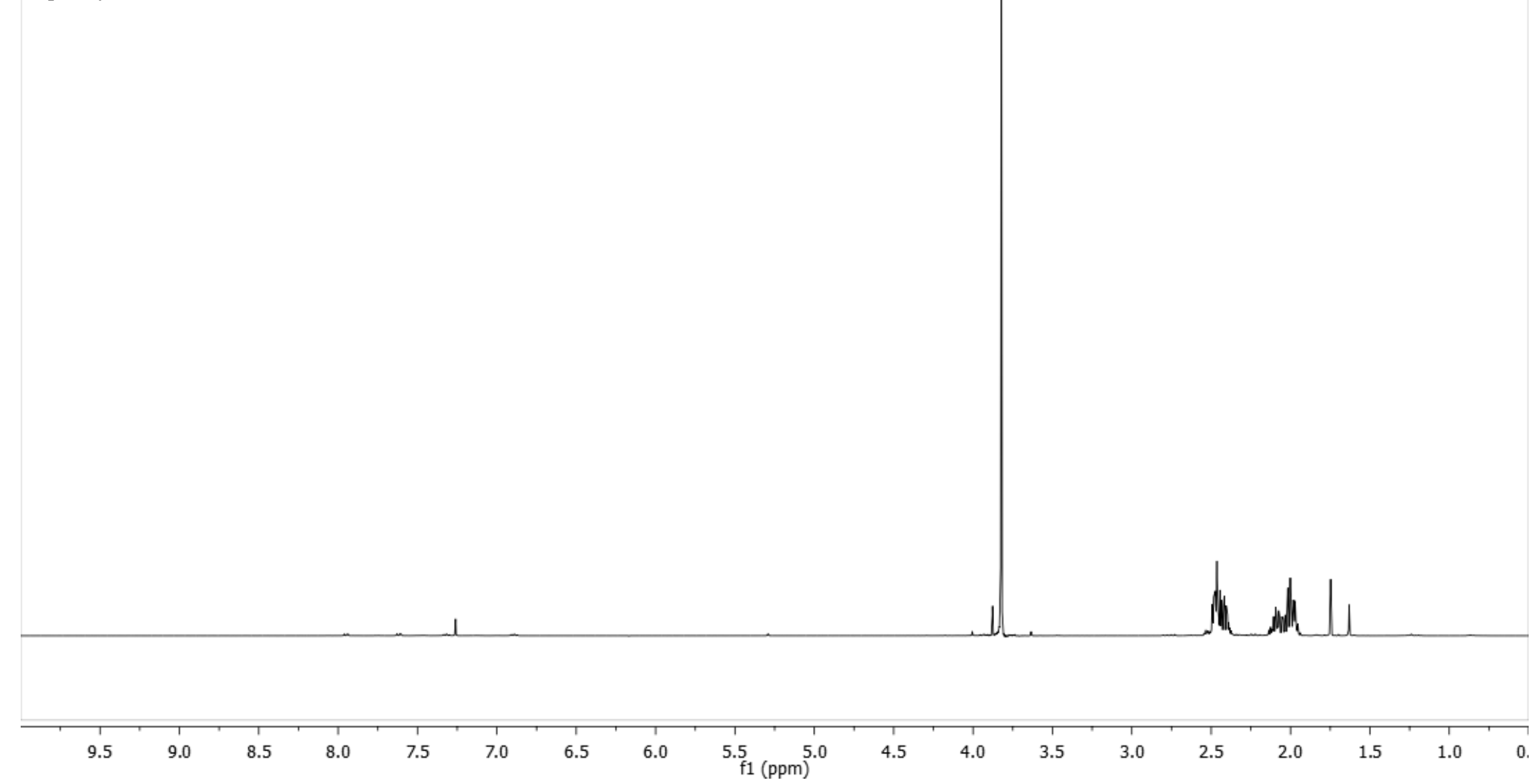



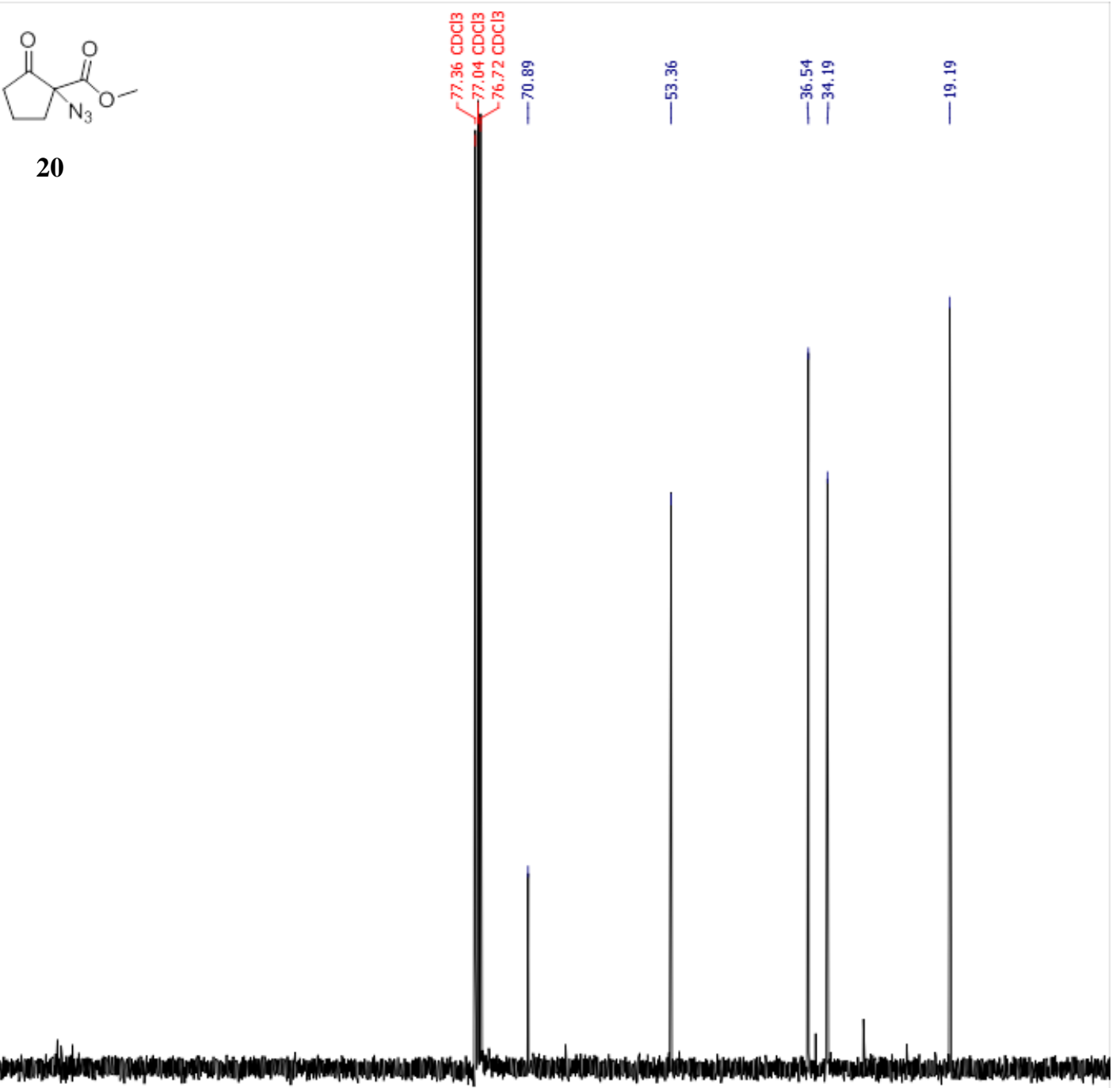

${ }^{13} \mathrm{C}$ NMR

Solvent: $\mathbf{C D C l}_{\mathbf{3}}$

Frequency: $100.6001 \mathrm{MHz}$
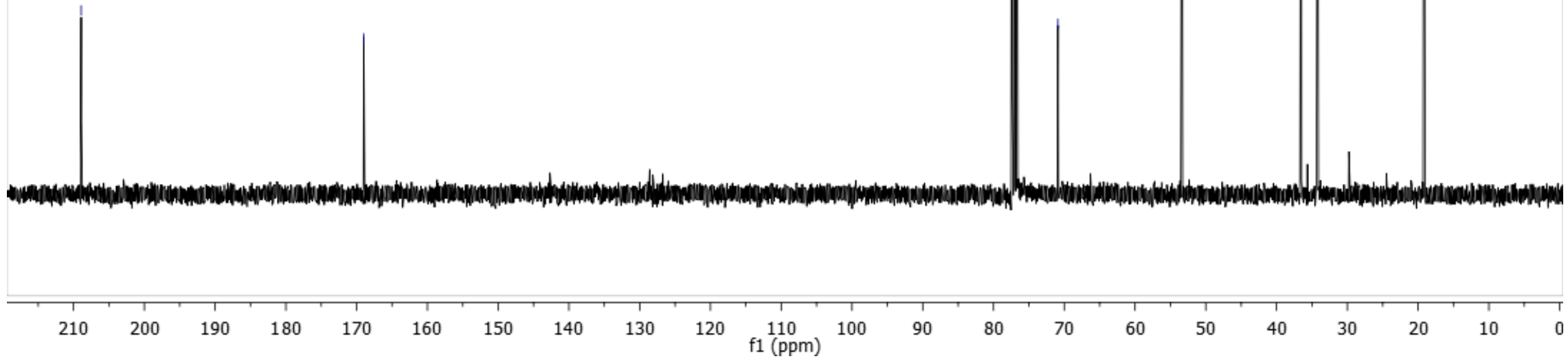


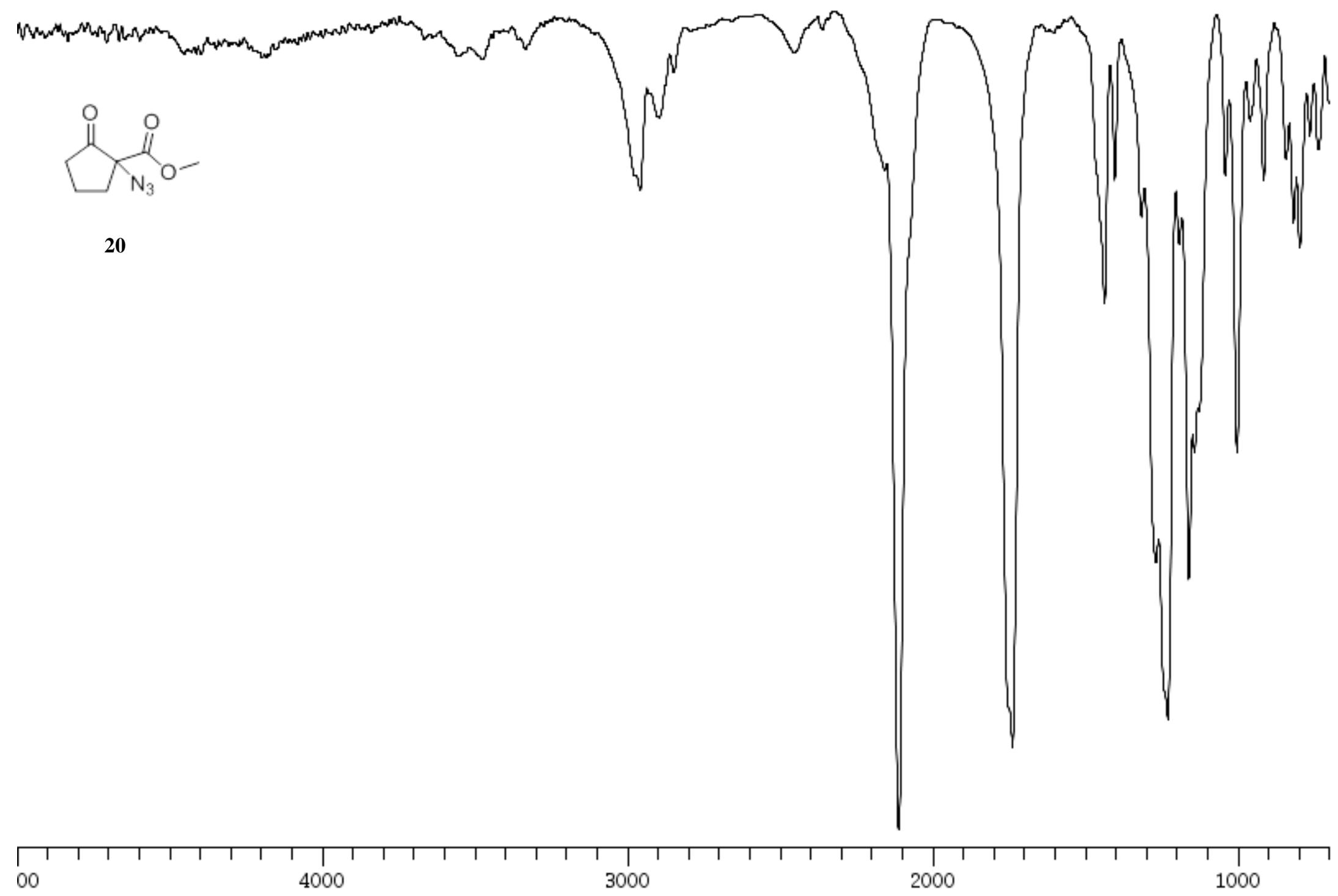

